\title{
Measurement of the Polarized \\ Forward-Backward Asymmetry \\ of $B$ Quarks Using Momentum-Weighted \\ Track Charge at SLD
}

Thomas Robert Junk

SLAC-Report-95-476

November 1995 


\section{DISCLAMMER}

Portions of this document may be illegible in electronic image products. Images are produced from the best available original document. 
This document and the material and data contained therein, was developed under sponsorship of the United States Government. Neither the United States nor the Department of Energy, nor the Leland Stanford Junior University, nor their employees, nor their respective contractors, subcontractors, or their employees, makes any warranty, express or implied, or assumes any liability or responsibility for accuracy, completeness or usefulness of any information, apparatus, product or process disclosed, or represents that its use will not infringe privately-owned rights. Mention of any product, its manufacturer, or suppliers shall not, nor is it intended to, imply approval, disapproval, or fitness for any particular use. A royalty-free, nonexclusive right to use and disseminate same for any purpose whatsoever, is expressly reserved to the United States and the University. 


\section{MEASUREMENT OF THE POLARIZED \\ FORWARD-BACKWARD ASYMMETRY \\ OF $B$ QUARKS USING MOMENTUM-WEIGHTED \\ TRACK CHARGE AT SLD*}

\section{Thomas Robert Junk}

Stanford Linear Accelerator Center

Stanford University, Stanford, CA 94309

\section{SLAC-REPORT-95-476}

November 1995

Prepared for the Department of Energy

under contract number DE-AC03-76SF00515

Printed in the United States of America. Available from the National Technical Information Service, U.S. Department of Commerce, 5285 Port Royal, Springfield, Virginia 22161

Ph.D. thesis, Stanford University

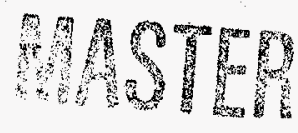

Abstract

This thesis presents a direct measurement of the parity-violating parameter $A_{b}$ by analyzing the polarized forward-backward asymmetry of $b$ quarks in $e^{+} e^{-} \rightarrow Z^{0} \rightarrow \dot{b} \bar{b}$. Data were taken at the Stanford Linear Accelerator Center (SLAC), with the Stanford Large Detector (SLD), which records the products of $e^{+} e^{-}$interactions at a center of mass energy $\sqrt{s}=91.2 \mathrm{GeV} / \mathrm{c}^{2}$ at the SLAC Linear Collider (SLC). The SLC/SLD experimental apparatus provides a unique and ideal environment for measuring electroweak asymmetries. Heavy flavor decays of the $Z^{0}$ were identified inclusively by taking advantage of the long lifetime of $B$ hadrons, the small, stable SLC beam spot, and SLD's precise tracking detectors. Two analysis techniques for measuring $A_{b}$ are presented: a binned fit to the left-right forward-backwards asymmetry of tagged events signed with momentum-weighted track charge, and a selfcalibrating maximum-likelihood technique using momentum-weighted charge from the two hemispheres in each tagged event. From our 1994-1995 sample of $3.6 \mathrm{pb}^{-1}$, having a luminosity-weighted average $e^{-}$polarization of $77.3 \%$, and our 1993 sample of $1.8 \mathrm{pb}^{-1}$, having a luminosity-weighted polarization of $63.1 \%$, we obtain $A_{b}=$ $0.848 \pm 0.046$ (stat.) \pm 0.050 (syst.). 


\section{Acknowledgements}

Behind every measurement in particle physics these days is the work of many people doing an enormous variety of tasks. With these people I have worked, played, and learned much, and I owe a deep debt of gratitude to each one. I must tender my apologies in advance for my deserving colleagues I have mistakenly left out.

First, I would like to thank my advisor, Rafe Schindler, for encouraging me to take part in the SLD experiment. His leadership example in the SLD heavy flavors working group inspired us all. His insistence on excellence in detector work and physics analysis has left its mark on many topics pursued at SLD, including this one. I have learned much as a graduate student, and Rafe has played an important role in my graduate experience.

For his courage and strength in the face of adversity, I thank Marty Breidenbach. It was his perserverance which kept SLD together and active, and which has paid off handsomely in physics results. And also for his kindness toward graduate students, I give him my deepest gratitude. Thanks go also to Charlie Baltay, our co-spokesman and statesman-at-large, who keeps SLD on the world map.

Special thanks go to Bruce Schumm, whose guidance and suggestions are integral to this thesis. Bruce shows by example and by critical participation, how careful physics analyses are done, and it is my hope to live up to those exacting standards in what I do. I would also like to thank Bruce for his unique sense of humor, which has lightened many a dark night at the terminal and on the experiment. I would like to thank Su Dong for his support of the heavy flavor physics group, his excellent troubleshooting ability, and his acumen with intricate physics topics. Su Dong always gave us something interesting to think about.

I $\neq$ has been a joy to work with the Compton polarimeter gang and the electroweak group - Mike Woods, Morris Swartz, Charlie Prescott, Peter Rowson, Mike Fero, Bruce Schumm, Dave Calloway, Eric Torrence, Ross King, Amit Lath, Ray Frey, Ken Moffeit, and Achim Wiedemann. Also many thanks to Guy Blaylock, whose beam energy measurements proved to be valuable diagnostics. It has also been a pleasure to work with the calorimeter crew - Iris Abt, Eric Vella, Tony Waite, Bill Wisniewski,
Andy Bazarko, Peter Rowson, Behzad Sanii, and Brooks Collins. And the interface between the hardware, the data, the tapes, and the folks on shift, the online group: Jim Russell, Mike Huffer, Joanne Bogart, Dave Sherden, Owen Saxton, and Jack Hoeflich.

I don't think anyone on SLD had a better office companion than myself. Homer Neal has provided boundless energy, dedication, and good cheer to everything he does. His work on tagging $Z^{0} \rightarrow b \bar{b}$ events has been used in the analysis presented in this thesis. I'll also relish my times with my fellow graduate students Mike and Holly Hildreth, Dave Williams, Tom Pavel, Jon Labs, Fritz DeJongh, Bijan Nemati, Pablo Saez, Matt Strassler, Jared Levy, Alex Hsieh, Chris Perez, Amanda Peet, Angela Cotera, Mary Jane Adriaans, Jie Zheng, Max Allen, Mark Ndesandjo and Mike Condron.

Special thanks to the SLAC theory group, particularly Michael Peskin, Joanne Hewett, and Tom Rizzo, who inspire us and give us renewed interest in our measurements.

I am positive the SLD collaboration would not function at all without Barbara Barrera, Margaret Helton, and Mary Brau; certainly not nearly as well without the good cheer and good sense they possess. The SLAC illustrations department has been invaluable in providing many illustrations used in this thesis.

The whole SLD gang have been great pals and guides these years - Tony Johnson, Richard Dubois, Terry Schalk, Len Moss, Karen Heidenreich, Jim Brau, Jenny Huber, Sarah Hedges, Bob Messner, Geordie Zapalac, Steve Wagner, Phil Burrows, Chris Dammerell, John Jaros, Bill Ash, Steve Manly, Dave Muller, Jaroslav Va'Vra, Tom Markiewicz, Leon Rochester, Tracy Usher, Joe Perl, Gary Bower, Hiro Masuda, Mary King, Takashi Muryama, Henry Band, and Dick Prepost. Thanks also to my friends Kevin Pitts, Eric Weiss, John Coller, Eric Church, Anthony Szumilo, Jingchen Zhou, Hyejoo Kang, Cheng Gang Fan, John Yamartino, Saul Gonzales, Nancy Mar, Donatella Falciai, Victor Serbo, Aaron Chou, Nety Krishna, Stefane Willocq, Xiaoqing Yang, Hwanbae Park, Ken Baird, and Peter Tenenbaum.

The SLC has been a difficult accelerator to commission and operate. The efforts of the physicists, operators, and technical staff have been heroic, and have brought 
about the success of the program. Special thanks to Nan Phinney, Mark Ross, Paul Emma, Franz-Joseph Decker, and the whole gang.

I would also like to thank my family for their support and encouragement through these years - My father William, my mother Lucille, and my brother Kenneth.

And lastly, I would like to thank Ann Williamson for her patience and support through these times that have been alternately trying and exhilirating.

Many heartfelt thanks to all the hard-working and fun people I have had as friends these past years.

\section{Contents}

1 Introduction 1

1.1 Introduction to the Standard Model . . . . . . . . . . . . 2

1.2 The Electroweak Interaction . . . . . . . . . . . . 5

1.2.1 The Electromagnetic Interaction . . . . . . . . . . . . 5

1.2 .2 Unification With the Weak Force . . . . . . . . . . 6

1.2.3 Spontaneous Symmetry Breaking and Gauge Boson Masses . . 9

1.3 The Strong Interaction . . . . . . . . . . . . . . . . 12

1.4 Tree-Level Asymmetries at the $Z^{0} \ldots \ldots \ldots \ldots \ldots$

1.4.1 The Left-Right Production Asymmetry $A_{L R} \ldots \ldots \ldots 22$

1.4.2 Unpolarized Forward-Backward Asymmetries $A_{F B}^{f} \ldots \ldots . .23$

1.4.3 Forward-Backward Tau Polarization Asymmetry $A_{F B}^{P(\tau)} \ldots \ldots 25$

1.4.4 Polarized Forward-Backward Asymmetries $\tilde{A}_{F B}^{f} \ldots \ldots \ldots$

1.5 Radiative Corrections . . . . . . . . . . . . . . . 27

1.5 .1 Bremsstrahlung . . . . . . . . . . . . . . . 27

1.5.2 Vertex and Propagator Corrections . . . . . . . . . . 30

1.6. Sensitivity of $A_{b}$ to New Phenomena . . . . . . . . . . 32

1.6.1 Additional Z Bosons . . . . . . . . . . . . . . 33

1.6 .2 Minimal Supersymmetric Model . . . . . . . . . . . . . 33

1.6 .3 Anomalous Couplings . . . . . . . . . . . . . . . 34

2 Current Experimental State $\quad 36$

2.1 Measurements of $A_{b}$ With Leptons ... . . . . . . . . . 36

2.2 Measurements of $A_{b}$ With Momentum-Weighted Charge . . . . . . 38 
2.3 Combined LEP $A_{b} \ldots \ldots \ldots \ldots \ldots \ldots$

$2.4 A_{F B}^{b}$ at Other Energies . . . . . . . . . . . . . 40

3 SLC and the Compton Polarimeter $4 \mathbf{4 2}$

3.1 The SLAC Linear Collider . . . . . . . . . . . . . . . . . . 42

3.2 Performance . . . . . . . . . . . . . . . 48

3.3 Linear Colliders and Synchrotrons . . . . . . . . . . . . . 50

3.4 Spin Production and Transport ................ 53

3.4 .1 Electron gun . . . . . . . . . . . . . . 53

3.4 .2 Photocathode .................... 54

3.4.3 Electron source optics . . . . . . . . . . . . . 57

3.4.4 Damping Ring Spin Transport . . . . . . . . . . . . 57

3.4 .5 Arc Spin Transport . . . . . . . . . . . . . . . . 59

3.5 Spin Measurement . . . . . . . . . . . . . . 62

3.5.1 Compton Polarimeter . . . . . . . . . . . . . . . 62

3.5 .2 Møller Polarimeter . . . . . . . . . . . . . . . 74

3.5.3 Energy Spectrometer . . . . . . . . . . . . . . 77

3.6 Summary ............................. 79

4 Experimental Apparatus: SLD $\quad \mathbf{8 0}$

4.1 Luminosity Monitor . . . . . . . . . . . . . . . . . . . . . . . . . . . . 80

4.2 Tracking: Central Drift Chamber . . . . . . . . . . . . 81

4.2 .1 Mechanical Design . . . . . . . . . . . . . . 82

4.2 .2 CDC Reconstruction . . . . . . . . . . . . . 85

4.2 .3 CDC Performance ...................... 87

4.3 Tracking: Vertex Detector . . . . . . . . . . . . . 93

4.3.1 Mechanical Design ... . . . . . . . . . . . . 93

4.3 .2 VXD Reconstruction . . . . . . . . . . . . . . . . . . . . . . . .

4.3 .3 VXD Performance . . . . . . . . . . . . . 97

4.4 Particle ID: Cherenkov Ring Imaging Detector . . . . . . . . . . . 100

4.5 Calorimetry: Liquid Argon and WIC Pads . . . . . . . . . . . . 102

4.6 Muon Identification: WIC Strips . . . . . . . . . . . . . . 106
5 The SLD Monte Carlo $\quad 108$

5.1 Introduction . . . . . . . . . . . . . . . 108

5.2 The Event Generator . . . . . . . . . . . . . . . . . 109

5.3 Mixing and Decay of $B$ Mesons . . . . . . . . . . . . 110

5.3 .1 Semileptonic Decays . . . . . . . . . . . . . . . . 111

5.3 .2 Hadronic Decays . . . . . . . . . . . . . . . . . . 113

5.4 Decays of Charmed Mesons . . . . . . . . . . . 115

5.5 Detector Simulation . . . . . . . . . . . . . . . 115

5.5 .1 Detector $/$ Particle Interactions . . . . . . . . . . . . . . . . . . . . . . . . . . . .

5.5 .2 Digitization . . . . . . . . . . . . . . 120

5.5 .3 Background Overlay . . . . . . . . . . . . . 122

$6 \quad A_{b}$ with Momentum-Weighted Charge 123

6.1 Introduction . . . . . . . . . . . . . . . 123

6.1 .1 Technique Overview . . . . . . . . . . . . . 124

6.1.2 Why Momentum-Weighted Charge Works . . . . . . . . 127

6.2 Experimental Results . . . . . . . . . . . . . . . . . . 128

6.2 .1 Event Selection . . . . . . . . . . . . . . . . 128

6.2 .2 Trigger and $Z^{0}$ Filter . . . . . . . . . . . . . . . . . . . . . . . . . . . . . . . . . . .

6.2 .3 Analysis Requirements . . . . . . . . . . . . . . . . 130

6.2 .4 B Tagged Sample . . . . . . . . . . . . . . . . 136

6.2.5 Systematic Uncertainties on the B Tag . . . . . . . . . 142

6.2.6 Momentum-Weighted Charge Distributions . . . . . . . . . 144

6.2 .7 Asymmetry Fit . . . . . . . . . . . . . . . . . 146

6.3 Systematic Error Analysis . . . . . . . . . . . . . . . 150

6.3 .1 Detector Modeling . . . . . . . . . . . . . . 152

6.3.2 Modeling Physical Processes . . . . . . . . . . . . . . 164

6.3 .3 Monte Carlo Statistics . . . . . . . . . . . . . . . . . 174

6.4 Cross-Checks . . . . . . . . . . . . . . . . . . . 174

6.4 .1 Kappa dependence ... . . . . . . . . . . . . . . 174

6.4 .2 Tag dependence . . . . . . . . . . . . . . 176 
6.4.3 Hemisphere consistency . . . . . . . . . . . . . 177

6.5 Summary ........................... 178

$7 A_{b}$ Analysis: Maximum Likelihood $\quad \mathbf{1 7 9}$

7.1 General Formulation . . . . . . . . . . . . . . . . . 180

7.2 Likelihood Function . . . . . . . . . . . . . . . . 181

7.3 Calibrating the Analyzing Power . . . . . . . . . . . . 182

7.3 .1 Ansatz . . . . . . . . . . . . . . . 182

7.3.2 Correct-Sign Probability . . . . . . . . . . . . . . 184

7.3.3 Hemisphere Correlations . . . . . . . . . . . . . . . . 188

7.3 .4 Light-Flavor Subtraction . . . . . . . . . . . . . . . 191

7.3.5 Checking the Gaussian Hypothesis . . . . . . . . . . . . 192

7.3 .6 QCD Corrections . . . . . . . . . . . . . . . 195

7.4 Likelihood fit to MC . . . . . . . . . . . . . . . 196

7.5 Measurement of $A_{b}$ with Maximum Likelihood Track Charge . . . . . 196

7.6 Systematic Error Analysis . . . . . . . . . . . . . . . . . . 198

7.6.1 Statistical Error on $\alpha_{b} \ldots \ldots \ldots \ldots \ldots$

7.6 .2 Shape of the $Q_{b}$ distribution . . . . . . . . . . . . . . 199

7.6.3 Angular Dependence of $\alpha_{b} \ldots \ldots \ldots \ldots$. . . . . . . 199

7.6.4 Interhemisphere Correlation . . . . . . . . . . . . 200

7.6 .5 Light Flavor Subtraction . . . . . . . . . . . . . . . . . 202

7.6 .6 Detector material . . . . . . . . . . . . 203

7.6 .7 Light-flavor coupling asymmetries . . . . . . . . . . . . . 204

7.6 .8 Tag purity . . . . . . . . . . . . . . . . . . 205

7.6 .9 QCD correction . . . . . . . . . . . . 206

7.7 Normalized Momentum-Weighted Charge . . . . . . . . . . . . 206

7.8 Summary . . . . . . . . . . . . . . . . 207

8 Global Fit to $Z b b$ Vertex Parameters $\quad \mathbf{2 1 0}$

8.1 Independent Parameters . . . . . . . . . . . . . . 210

8.2 Fit Inputs . . . . . . . . . . . . . . . . . . . . 212

8.3 Constraints on Parameters . . . . . . . . . . . . . 213
9 Summary and Outlook $\quad \mathbf{2 1 5}$

9.1 Results and Comparison . . . . . . . . . . . . . 215

9.2 Track Charge Prospects . . . . . . . . . . . . . . . 217

9.3 New Techniques . . . . . . . . . . . . . . . . . . . . . 218

9.4 Calibrating New Techniques . . . . . . . . . . . . . . . 219

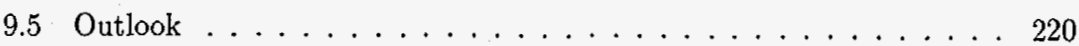

$\begin{array}{lr}\text { A SLD Collaboration } & 221\end{array}$

B Compton Online Software

B.1 Structure and Requirements . . . . . . . . . . . . . . 225

B.2 Raw Data . . . . . . . . . . . . . . . . . 228

B.3 Summed Data . . . . . . . . . . . . . . . . . . . 228

B.4 Scans . . . . . . . . . . . . . . . . . . . . 232

C CDC Vector Hit Finder $\quad \mathbf{2 3 4}$

C.1 The need for Vector Hits . . . . . . . . . . . . . . 234

C.2 Algorithm . . . . . . . . . . . . . . . . . 235

C.3 Performance . . . . . . . . . . . . . . 238

D Trigger and Filter $\quad \mathbf{2 4 1}$

D.1 Trigger . . . . . . . . . . . . . . . . . . . . 241

D.2 Z Filters . . . . . . . . . . . . . . . . . . . . . 243

D.2.1 The EIT Calorimetric Filter . . . . . . . . . . . . 243

D.2.2 Additional Filters: Tau, Mu Pair, and KZ0F . . . . . . . 244

E Monte Carlo Charm Decays

245 


\section{List of Tables}

1.1 Fermion constituents of the Standard Model . . . . . . . . . . . 4

1.2 Fermion quantum numbers . . . . . . . . . . . . . .

1.3 Chiral couplings of the standard fermions to the $\gamma$ and $Z^{0} \ldots \ldots 19$

$1.4 \quad$ Values of $A_{f}$ for $\sin ^{2} \theta_{W}=0.23 \ldots \ldots \ldots 21$

2.1 Current leptonic measurements of $A_{e}$ used to extract $A_{b}$ from $A_{F B}^{b}$. From Reference $[64] . \ldots \ldots \ldots \ldots$

2.2 Measurements from the four LEP experiments of $A_{F B}^{b}$ from semileptonic $B$ decays, given in Reference [65], uncorrected for QCD, QED, and $\gamma-Z$ interference. The values of $A_{b}$ are extracted using the value of $A_{e}$ from Table 2.1, and are also corrected for radiative effects and photon exchange. . . . . . . . . . . . . .

2.3 Measurements from three of the four LEP experiments of $A_{F B}^{b}$ using momentum-weighted charge in lifetime-tagged $Z^{0} \rightarrow b \bar{b}$ samples, as reported at the 1995 Europhysics Conference [65]. The values of $A_{b}$ extracted assume $A_{e}=0.1506 \pm 0.0028 \ldots \ldots \ldots \ldots \ldots$

2.4 Measurements of $A_{F B}^{b}$ at $\sqrt{s}=57.8 \mathrm{GeV}$ from the three TRISTAN experiments. Correction for $B$ meson mixing is not included in these measurements, but would enlarge the values by a factor of $\sim 1.3$ if it were. . . . . . . . . . . . . . . . 40

3.1 Parameters of the SLC damping rings [76][77] . . . . . . . . . . 46

3.2 SLC performance parameters for 1993-1995. . . . . . . . . 79

4.1 Vertex detector design parameters. . . . . . . . . . . . . . . 94

4.2 Geometrical properties of the LAC and WIC pads . . . . . . . . 10
Mixing parameters of the SLD Monte Carlo. . . . . . . . . . 110

SLD semileptonic $B$ meson decay branching modes. . . . . . . . . . 111

Relative fractions of different $D^{* *}$ states generated in $B$ decay in the SLD Monte Carlo. Fractions originate from the CLEO Monte Carlo $[123] \ldots \ldots \ldots \ldots \ldots \ldots \ldots \ldots$

5.4 CLEO decay model hadronic tuning parameters. . . . . . . . . . . 114

5.5 Comparison of inclusive multiplicities of $\pi^{ \pm}, K^{ \pm}$, and $P / \bar{P}$ between the CLEO/SLD decay model and ARGUS measurements [124],[125]. 114

5.6 Comparison of branching fractions of $B$ mesons to the different $D$ mesons. . . . . . . . . . . . . . . . . . . . . . . 114

6.1 Systematic errors of the $B$ Tag composition $\ldots \ldots \ldots . \ldots 143$

6.2 Details of the binned asymmetry fit for $A_{b} \ldots \ldots \ldots \ldots$. . . . . 151

6.3 Systematic uncertainties on the electron beam polarization as measured by the Compton Polarimeter [42]. The later $2 / 3$ of the 1993 running benefited from routine laser polarization scanning and has smaller errors. . . . . . . . . . . . . . . . . . 161

6.4 Generator-level analyzing powers for events with different numbers of fragmentation protons. . . . . . . . . . . . . . 165

6.5 Generator-level analyzing powers for events with different numbers of fragmentation $K^{ \pm} \ldots \ldots \ldots \ldots \ldots \ldots \ldots$

6.6 Summary of the detector modeling and general systematics of the binned asymmetry fit to $A_{b} \ldots \ldots \ldots \ldots \ldots$

6.7 Summary of the $B$ hadron fragmentation systematic errors of the binned asymmetry fit to $A_{b} \ldots \ldots \ldots \ldots \ldots \ldots$

6.8 Summary of the $B$ hadron mixing and decay systematic errors of the binned asymmetry fit to $A_{b} \ldots \ldots \ldots \ldots \ldots$. . . . . . 169

6.9 Summary of the charmed hadron modeling systematic errors of of the binned asymmetry fit to $A_{b} \ldots \ldots \ldots \ldots \ldots$. . . . . . . 170

6.10 Hemisphere-consistency cross-check comparison between data and Monte Carlo. . . . . . . . . . . . . . . . . . 178 
7.1 Contributions to the light-flavor subtraction to the data $\sigma_{\text {sum }}$ and

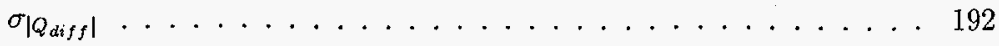

7.2 Fit biases estimated from a Monte Carlo simulation with $Q_{b}$ gener ated with different distributions. . . . . . . . . . . . . . . 193

7.3 Details of the momentum-weighted charge maximum-likelihood fit for

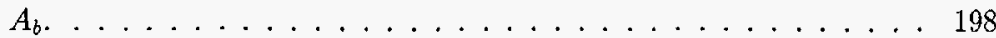

7.4 Differences between the hemisphere correlations and their effects on the measured value of $A_{b} \ldots \ldots \ldots \ldots 20 \ldots$

7.5 Systematic error summary for the self-calibrated maximum-likelihood measurement of $A_{b} \ldots \ldots \ldots . \ldots . \ldots 207$

7.6 Details of the normalized momentum-weighted charge maximum-likelihood fit for $A_{b} \ldots \ldots \ldots \ldots \ldots \ldots$. . . . . . . . 208

8.1 Measured observables contributing to the global fit to $Z b b$ vertex parameters. . . . . . . . . . . . . . . . . . 212

B.1 Contents of the raw Compton polarimeter data structure. . . . . . 231

C.1 Percentages of found Vector Hits, listed by the number of total hits and the number of correct hits, indicating the purities of Vector Hits with different lengths. . . . . . . . . . . . . . . . . 240

D.1 Description of the SLD triggers. . . . . . . . . . . . . . 242

D.2 LAC layer thresholds and online energy conversions. . . . . . . . . 242

E.1 SLD decay table for the $D^{*+}$ meson. . . . . . . . . . . 245

E.2 SLD decay table for the $D^{* 0}$ meson. . . . . . . . . . . 245

E.3 SLD decay table for the $D^{0}$ meson. . . . . . . . . . . . 246

E.4 SLD decay table for the $D^{+}$meson. . . . . . . . . . . . 247

E.5 SLD decay table for the $D_{s}^{+}$meson, Part $1 \ldots \ldots \ldots \ldots 248$

E.6 SLD decay table for the $D_{s}^{+}$meson, Part 2. . . . . . . . . . . . . 249

E.7 SLD decay table for the $\Lambda_{c}^{+}$baryon, Part 1. . . . . . . . . . 250

E.8 SLD decay table for the $\Lambda_{c}^{+}$baryon, Part $2 \ldots \ldots \ldots 251$

\section{List of Figures}

1.1 Vertex diagrams in QCD. . . . . . . . . . . . . . 14

1.2 The fragmentation functions for $u d s, c$, and $b \ldots \ldots 17$

1.3 Tree-level electroweak contributions to $e^{+} e^{-} \rightarrow f \bar{f}$ in the Standard Model. . . . . . . . . . . . . . . . . . . . . 19

1.4 Dependence of the asymmetries $A_{L R}, A_{L R}^{b}$, and $A_{F B}^{b}$ on the center-of mass energy. . . . . . . . . . . . . . . . . . . . 28

1.5 Lowest-order bremsstrahlung contributions to $e^{t} e^{-} \rightarrow Z^{0} \rightarrow q \ddot{q} . \quad \ldots 28$

1.6 Corrections to the $Z^{0}$ lineshape from ISR. The uncorrected cross section is denoted $\sigma_{0}$. From reference [52]. . . . . . . . . 29

1.7 Leading order QED and QCD vertex corrections. . . . . . . . 31

1.8 Propagator corrections to the electroweak neutral current. . . . . 31

1.9 Vertex corrections to $Z^{0} \rightarrow b \bar{b}$ within the Standard Model . . . . . . 32

1.10 Chargino diagrams $(\mathrm{a}-\mathrm{c})$ and neutralino diagrams $(\mathrm{d}-\mathrm{f})$ contributing to $Z^{0} \rightarrow b \bar{b}$ in the Minimal Supersymmetric Model. . . . . . . . 34

1.11 Contours showing 100 times the contribution to $A_{b}$ from neutralinos, for $\tan \beta=70$. From Boulware and Finnell [62]. . . . . . . 35

1.12 Corrections to $R_{b}$ and $A_{b}$ from charged and neutral scalars for $\tan \beta=$ 70. From Boulware and Finnell [62]. . . . . . . . . . . . 35

2.1 Measurements of $A_{F B}^{b}$ as a function of $E_{C M}$, measured at PEP, PETRA, TRISTAN, and LEP. . . . . . . . . . . . . . 41

3.1 Layout of the spin-polarized SLC. . . . . . . . . . . . . . 43

3.2 The disk-loaded waveguide linac structure. . . . . . . . . . . . . 4

3.3 Integrated and differential luminosity histories of the SLC. . . . . 49 
3.4 History of the electron beam polarization as a function of the count of collected $Z^{0}$ s. . . . . . . . . . . . . . . . . . 50 The polarized gun used on SLC in $1993 . \ldots \ldots \ldots \ldots$ Energy levels in unstrained and strained GaAs. . . . . . . . . 55 Optical layout of the polarized source. . . . . . . . . . 56 Spin transport in the North Damping Ring . . . . . . . . . . 58 Spin Transport and Polarimeters at SLC . . . . . . . . . . . 61

3.10 The Compton Polarimeter in the South Final Focus . . . . . . . .

3.11 Schematic diagram of the Compton polarimeter optical layout. . . .

3.12 Lowest-order contributions to Compton scattering. . . . . . . . . . .

3.13 The asymmetry between the $J=3 / 2$ and $J=1 / 2$ states of Compton scattering as a function of the energy lost by the electron, compared against the measured asymmetry for $P_{e}=63 \%$. . . . . . .

3.14 A scan over the voltages of the CP and PS Pockels cells to determine the quarter-wave voltage and the unpolarized fraction. . . . . . . . .

3.15 A scan over the voltages of the CP and PS Pockels cells to determine the optimal phase of the light to measure the electron beam

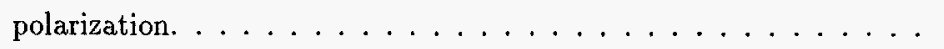

3.16 A fit to the measured signal and asymmetry (points) using the unbound electron hypothesis (histogram). Dashed lines indicate estimated background signal and asymmetry. From Reference [101]. . .

3.17 A fit to the measured signal and asymmetry (points) using the bound electron hypothesis (histogram). Dashed lines indicate estimated background signal and asymmetry. From Reference [101]. . . . . . 76

3.18 The Wire Imaging Synchrotron Radiation Detector (WISRD) . . . . 78

4.1 Quadrant view of SLD, showing the precision tracking, particle identification, calorimetry, and muon identification subsystems. . . . . 81

4.2 Cross-section of a portion of the CDC in the plane perpendicular to the beam axis. . . . . . . . . . . . . . . . 83

4.3 The field in a single half-cell of the CDC . . . . . . . . . .

4.4 Charged track multiplicity distribution for data and Monte Carlo. . showing an overall inefficiency, but also an excess at high momentum. 88 
6.4 The first four track selection variables. . . . . . . . . . . 132

6.5 The second four track selection variables. . . . . . . . . . . 133

6.6 Event selection variables. . . . . . . . . . . . . . . . 134

6.7 Distributions of vertexing-quality track cut variables. . . . . . . 138

6.8 Comparison of the normalized $2 D$ impact parameter in data (points) and Monte Carlo (histogram). . . . . . . . . . . . . . . . . . 139

6.9 Normalized 2-D impact parameter by flavor. . . . . . . . . . . . 140

6.10 An event tagged with the normalized 2-D impact parameter tag. . . 141

6.11 Performance of the 2-Dimensional Normalized Impact-Parameter Tag 142

6.12 Momentum-Weighted Charge comparison of data and Monte Carlo for tagged events. . . . . . . . . . . . . . . 145

6.13 Distribution of $Q_{\text {sum }}$ for tagged events, compared between data and Monte Carlo. . . . . . . . . . . . . . . . . . . 146

6.14 The Calorimetric thrust axis $\cos \theta_{T}$, signed with momentum-weighted track charge, binned separately for events created with the lefthanded and right-handed $e^{-}$beams. . . . . . . . . . . . 147

6.15 Binned fit of the MC asymmetry to the data. . . . . . . . . . . 148

6.16 Total momenta of positive and negative muons separately as a function of $\cos \theta \ldots \ldots \ldots \ldots \ldots \ldots \ldots \ldots \ldots$

6.17 Data and Monte Carlo distributions of gamma conversions and their asymmetries. . . . . . . . . . . . . . 156

6.18 Comparison of data and Monte Carlo track $\cos \theta$, in bins of calorimeter thrust $\cos \theta_{r} \ldots \ldots \ldots \ldots \ldots \ldots \ldots \ldots$

6.19 QCD corrections as calculated in [58], compared against those estimated from JETSET 7.4. . . . . . . . . . . . . . 160

6.20 Analyzing power vs. $\kappa \ldots \ldots \ldots \ldots \ldots$

6.21 Dependence of the value of $A_{b}$ measured with the binned asymmetry fit on the momentum-weighting exponent $\kappa \ldots \ldots \ldots 175$

6.22 Tag dependence of the binned asymmetry $A_{b}$ measurement. . . . . 176

7.1 Monte Carlo distributions of $Q_{b}$ and $Q_{\bar{b}}$, with fits to a Gaussian hypothesis. The joint probability distribution has a correlation of $\sim 3 \% .185$ 1995 data with $\kappa=0.5 \ldots \ldots \ldots \ldots$. . . . . . . . 189

7.4 Possible effects of correlation on $\mathrm{p}\left(Q_{b}, Q_{\bar{b}}\right) \ldots \ldots \ldots \ldots$

7.5 Different hypothetical $Q_{b}$ distributions used to test the sensitivity of the fit to $p\left(Q_{b}\right) \ldots \ldots \ldots \ldots \ldots \ldots$. . . . . . . . 194

7.6 Gluon bremsstrahlung smearing the angle of the final-state quark (left), and backscattering it into the opposite hemisphere (right). . . 195

$7.7 \quad \log$ likelihood as a function of $A_{b} \ldots \ldots \ldots \ldots$

7.8 Trial shapes for the $\cos \theta$-dependence of $\alpha_{b} \ldots \ldots \ldots 200$

7.9 Comparison of generator-level correlations in the JETSET 7.4 string fragmentation model, JETSET's independent fragmentation option, and HERWIG 5.7. . . . . . . . . . . . . . . . . 201

7.10 Effect of detector material on the joint probability distribution of $Q_{b}$

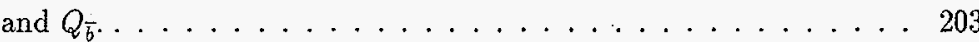

7.11 Tag dependence of the maximum-likelihood $A_{b}$ measurement. . . . . 205

7.12 Correct-sign probability estimate for normalized momentum-weighted charge. . . . . . . . . . . . . . . . . . 208

8.1 Combined constraints on the $b$ rate-like parameter $\xi_{b}$ shown with $\delta s^{2}, 213$ 8.2 Combined constraints on the $b$ asymmetry-like parameter $\zeta_{6}$ shown

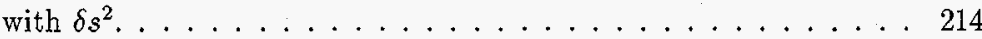

B.1 Schematic diagram of the Compton polarimeter data acquisition. . . 227

B.2 Raw signal observed in Channel 6 of the Cherenkov detector during clean running. . . . . . . . . . . . . . . . . . . 229

B.3 Raw signal observed in Channel 6 of the Cherenkov detector during dirty running ...................... . . 230

C.1 A vector hit with a noise hit replacing one of the true endpoints. . . 237

C.2 Performance of the CDC Vector Hit (VH) finder. . . . . . . . . . 239 


\section{Chapter 1}

\section{Introduction}

This thesis presents a measurement of the polarized forward-backward asymmetry of $b$-quark production in $e^{+} e^{-}$collisions at $\sqrt{s}=91.2 \mathrm{GeV}$. This asymmetry provides a direct measurement of parity violation in the electroweak couplings of the bottom quark, in particular the difference between the strengths of the couplings of lefthanded $b$ quarks and right-handed $b$ quarks to the $Z^{0}$ boson. The asymmetry is often referred to in the literature as $A_{b}$, and sometimes as $A_{b}^{L R}$ or $A_{L R}^{b}$.

The experimental determination of $A_{b}$ is under intense study worldwide, with efforts from SLC and the four LEP experiments; each year its measured value becomes more precise. Measuring $A_{b}$ constitutes a stringent test of the predictions of the Standard Model, and may either confirm our current understanding of the fundamental interactions between elementary particles, or help point the way to extending our models and deepening our knowledge. Particular interest is focused on $A_{b}$ at this time because recent, precise measurements of $R_{b}=\Gamma\left(Z^{0} \rightarrow b \bar{b}\right) / \Gamma\left(Z^{0} \rightarrow\right.$ hadrons $)$ show a noticeable discrepancy [1] with the prediction of the Standard Model and may be hinting at new phenomena modifying the interaction between the $Z^{0}$ and the $b$ quark.

This measurement was performed at the Stanford Linear Accelerator Center (SLAC), using data collected in 1993-1995 with the SLAC Large Detector (SLD) at the Stanford Linear Collider (SLC). SLC is the first linear $e^{+} e^{-}$collider and produces $Z^{0}$ bosons on resonance in collisions of polarized electrons with unpolarized positrons. The SLD is an ideal experiment for studying electroweak physics and the physics of heavy flavor for several reasons. Roughly $22 \%$ of hadronic $Z^{0}$ decays are decays into $b \bar{b}$, and the resulting $B$ hadrons receive a large boost in the laboratory frame of reference. The exceedingly small, stable beams of SLC, and the precision tracking performance of SLD allow analyses to take advantage of knowledge of both the production and decay points of long-lived heavy hadrons. The polarization of the electron beam consistently runs near $80 \%$, and its helicity may be aligned either along or opposite the direction of travel.

With these tools, a direct measurement of the parity-violating couplings of the $Z^{0}$ to the $b$ quark can be made. The remainder of this chapter presents this asymmetry in the context of the current understanding of elementary particle physics, and how a precise measurement of it may augment this understanding.

\subsection{Introduction to the Standard Model}

Physicists of the late $19^{\text {th }}$ century lived in a time in which nearly all observable phenomena could be accounted for with the existing models of the time: deterministic classical mechanics, Newtonian gravitation, Maxwell's recently unified description of electricity and magnetism, and thermodynamics. Only a few problems remained unexplained; three experimental, and one theoretical: radioactive decay, atomic line spectra, the blackbody radiation curve, and the description of propagating electromagnetic radiation in a moving frame. Investigation of these topics and the questions raised in the process opened the floodgates of physical discovery in the last one humdred years.

The road to our current model of the weak interactions began when Becquerel inadivertently left a photographic emulsion enclosed in a light-tight container under a sample of uranium salts and observed an exposure, thereby discovering spontaneous radioactive decay of heavy elements. In many ways, this interaction has been one of the more experimentally accessible ones and therefore one of the first observed, but also one of the most resistant to explanation. Weak interactions mediating nuclear decay take place on time scales easily measurable with ordinary clocks; the radioactive 
materials may be obtained from mines; and the detection apparatus is modest. At the time, neither the electron nor the nucleus had been discovered, so the origin of these rays remained a mystery.

In the mean time, investigations into the spectra of excited atomic hydrogen and the spectrum of thermal blackbody radiation gave birth in the 1920's to a quantum mechanical description of subatomic phenomena. Combining the formalism of quantum mechanics with special relativity in the early 1930's gave rise to the quantum field theory describing the interaction of charged particles and electromagnetic radiation, Quantum Electrodynamics (QED), to be described briefly below.

The observed spectrum of beta particles from radioactive decay of heavy nuclei was found to be continuous, which was not possible for a two-body decay of a heavy object. A third particle was introduced by Pauli, the neutrino, which would account for the missing energy and spin in beta decay [2]. Shortly thereafter, Enrico Fermi proposed a field-theoretic approach to describing beta decay by introducing a fourfermion vertex which coupled, for instance, a neutron, a proton, an electron, and a neutrino together at one point with a vector coupling. It was discovered that theories incorporating this vertex predicted infinite reaction probabilities once one-loop radiative correction calculations were attempted, even though experiments confirmed its tree-level predictions.

In 1956, Lee and Yang [3], after reviewing the literature, noticed that parity and charge-conjugation symmetries had not been checked in the weak interactions, and that they could possibly be violated. A series of experiments by Wu [4], Lederman. Garwin, and Schwartz [5], and Friedman and Telegdi [6] was conducted to test parity. conservation in nuclear $\beta$-decay and in $\pi^{ \pm}$and $\mu^{ \pm}$decay, finding that parity was maximally violated by charged weak currents. It was observed that these currents coupled only to left-handed fermions and right-handed antifermions. Maximal parity violation could easily be introduced into the Fermi model by substituting the vector coupling by a $V-A$ coupling at the four-fermion vertex.

Yang and Mills proposed in 1956 [7] a formulation of quantum field theory that allowed the introduction of non-Abelian gauge symmetry groups, an extension of the Abelian symmetries of QED. Glashow [8], Weinberg [9], and Salam [10] proposed
Table 1.1: Fermion constituents of the Standard Model

$$
\begin{aligned}
& \left(\begin{array}{c}
\nu_{e} \\
e
\end{array}\right)_{L}\left(\begin{array}{c}
\nu_{\mu} \\
\mu
\end{array}\right)_{L}\left(\begin{array}{c}
\nu_{\tau} \\
\tau
\end{array}\right)_{L} e_{R} \\
& \left(\begin{array}{c}
u \\
d
\end{array}\right)_{L}\left(\begin{array}{c}
c \\
s
\end{array}\right)_{L}\left(\begin{array}{c}
t \\
b
\end{array}\right)_{L} \begin{array}{llll}
u_{R} & c_{R} & t_{R} \\
d_{R} & s_{R} & b_{R}
\end{array}
\end{aligned}
$$

a model, described below, which incorporated both the electromagnetic and weak interactions as low-energy manifestations of a single interaction with $S U(2) \times U(1)$ gauge symmetry. The mediators of the electroweak force in this model are the photon $(\gamma)$, the heavy $Z^{0}$ boson, and the charged doublet $W^{ \pm}$.

At the outset, the Glashow-Weinberg-Salam model seemed beset with difficulties. It called for massive gauge bosons (mass terms could not be added to the Lagrangian and maintain gauge invariance), and it did not appear to be renormalizable. The first problem was addressed in Weinberg's paper [9] by incorporating the Higgs mechanism [11] of spontaneously breaking the symmetry of the theory in order to impart mass to the $W^{ \pm}$and the $Z^{0}$. The existence of a neutral, scalar Higgs boson remains unverified by experiment. Gerard t'Hooft solved [12] the renormalization problem in 1971, promoting the Weinberg-Salam model to a viable theory of electroweak interactions. The complete demonstration of the renormalizability of the electroweak theory requires fermions to be grouped in generations in order to cancel triangle anomalies.

Weak neutral currents, predicted by the Weinberg-Salam model, were first observed in 1973 [13][14] in the interactions $\bar{\nu}_{\mu} e \rightarrow \bar{\nu}_{\mu} e, \nu_{\mu} N \rightarrow \nu_{\mu} X$, and $\bar{\nu}_{\mu} N \rightarrow \bar{\nu}_{\mu} X$. Neutral currents are carried therefore by both the photon and the $Z^{0}$, and their amplitudes interfere.

A further vindication of the model came from the discovery of the $J / 2$; a $c \bar{c}$ bound state. The charm quark was predicted by the GIM mechanism [15] as an explanation for the lack of flavor-changing neutral currents in decays of the kaon. A 
third generation, heralded by the discovery of the $\tau$ lepton in 1975 [16], and filled in with the discovery of the $\Upsilon$ meson, a bound $b \bar{b}$ state, in 1977 [17], and finally the $t$ quark in 1995 [18][19], confirmed the basic doublet structure of fermions. In the gauge boson sector, the $W^{ \pm}$and the $Z^{0}$ were first identified in hadronic $p \bar{p}$ collisions at the $S P \bar{P} S$ collider at CERN in 1983 [20][21].

\subsection{The Electroweak Interaction}

The next two sections outline the electroweak sector of the Standard Model. Detailed presentations of this material may also be found in References [23], and [24], and [27]. This presentation roughly follows the notation and logic of Halzen and Martin [22].

\subsubsection{The Electromagnetic Interaction}

The most elegant formulations of physical models are the ones that start with the fewest postulates and describe the broadest range of observable behavior. Here we will start with the postulate of local gauge invariance and arrive at the formulation of QED. The Dirac equation for spin-1/2 particles,

$$
\left(i \gamma^{\mu} \partial_{\mu}-m\right) \psi=0
$$

can be obtained from the Lagrangian

$$
\mathcal{L}=i \bar{\psi} \gamma_{\mu} \partial^{\mu} \psi-m \bar{\psi} \psi
$$

This Lagrangian is already invariant under the transformation

$$
\psi(x) \rightarrow e^{i \alpha} \psi(x)
$$

where $\alpha$ is a constant. This global symmetry, through an application of Noether's theorem, requires the conservation of electric charge:

$$
\partial_{\mu} j^{\mu}=0
$$

where

$$
j^{\mu}=-e \bar{\psi} \gamma^{\mu} \psi
$$

If the phase $e^{i \alpha}$ is allowed to be a function of space and time, then the Lagrangian in Equation 1.2 is no longer invariant due to the presence of the derivative. The necessary modification to preserve local gauge invariance is to replace the derivative $\partial_{\mu}$ with a "covariant derivative" $D_{\mu}$ :

$$
\mathcal{L}=i \bar{\psi} \gamma_{\mu} D^{\mu} \psi-m \bar{\psi} \psi
$$

with

$$
D_{\mu}=\partial_{\mu}-i e A_{\mu}
$$

where the field $A_{\mu}$ transforms as

$$
A_{\mu} \rightarrow A_{\mu}+\frac{1}{e} \partial_{\mu} \alpha
$$

Finally, a photon kinetic energy term must be added, and local gauge invariance restricts its form to combinations of the field strength tensor $F_{\mu \nu}$. The QED Lagrangian then follows:

$$
\mathcal{L}=\bar{\psi}\left(i \gamma^{\mu} \partial_{\mu}-m\right) \psi+e \bar{\psi} \gamma^{\mu} A_{\mu} \psi-\frac{1}{4} F_{\mu \nu} F^{\mu \nu}
$$

with

$$
F_{\mu \nu}=\partial_{\mu} A_{\nu}-\partial_{\mu} A_{\mu} .
$$

This gauge symmetry precludes adding a term proportional to $A_{\mu} A^{\mu}$ and therefore requires that the photon be massless, unless the symmetry is broken by an interaction with another field. There currently is no experimental evidence for photon mass, and the upper bound is set at $3 \times 10^{-27} \mathrm{eV} / \mathrm{c}^{2}[25]$.

\subsubsection{Unification With the Weak Force}

Because the weak interaction couples left-handed particles within isodoublets (the right-handed versions of the fermions do not interact with the charged weak current), we seek a local gauge symmetry with the same symmetry as the spin- $\frac{1}{2}$ representation of the rotation group. The group $S U(2)$ is chosen for this; a convenient set of generators is the set of Pauli matrices $\sigma^{i}, i=1,2,3$. This symmetry is often referred 
to as $S U(2)_{L}$ because it describes the sector that interacts with only the left-handed fermions. The gauge transformation for the weak current then becomes

$$
\psi \rightarrow e^{i g \frac{\sigma^{i}}{2} \Lambda^{i}} \psi
$$

where the $\Lambda^{i}$ are three real functions of space and time and $g$ is a constant. As before, it is necessary to introduce new fields into the theory in order to preserve the gauge invariance of the derivative term. The covariant derivative has the form

$$
D_{\mu}=\partial_{\mu}-i g \frac{\sigma^{i}}{2} W_{\mu}^{i}
$$

where the three additional fields $W_{\mu}^{i}$ describe a massless isotriplet of gauge bosons Because the Pauli matrices $\sigma^{i}$ do not commute, the gauge transformation of the $W_{\mu}^{i}$ must include an extra term:

$$
W_{\mu}^{i} \rightarrow W_{\mu}^{i}+\partial_{\mu} \Lambda^{i}-g \vec{\Lambda} \times \vec{W}_{\mu} .
$$

The additional term adds an interaction between the gauge bosons. This feature is not surprising, since the weak charged bosons must interact with the photon at tree level. The kinetic energy term in the Lagrangian, $-\frac{1}{4} \vec{W}_{\mu \nu} \cdot \vec{W}^{\mu \nu}$, uses a form of the field strength tensor slightly modified from the QED case:

$$
W_{\mu \nu}^{i}=\partial_{\mu} W_{\nu}^{i}-\partial_{\nu} W_{\mu}^{i}-g \vec{W}_{\mu} \times \vec{W}_{\nu}
$$

The new fields $W_{\mu}^{i}$ interact with an isospin triplet of weal currents,

$$
J_{\mu}^{i}=\bar{\chi}_{L} \gamma_{\mu} \frac{\sigma^{i}}{2} \chi_{L}
$$

where $\chi_{L}$ is one of the left-handed isodoublets of Table 1.1

In addition to the $S U(2)_{L}$ symmetry, the $U(1)$ symmetry of QED is incorporated. Because weak neutral currents do not have a pure $V-A$ form, the neutral member of the weak isotriplet mentioned above cannot alone be the correct representation. Instead, a linear combination of the gauge boson of the $U(1)$ symmetry and the neutral member of the above isotriplet is sought. The generator of the $U(1)$ symmetry is then named the "weak hypercharge" $Y$, and is defined to be

$$
Y \equiv 2\left(Q-T^{3}\right)
$$

Table 1.2: Fermion quantum numbers. The second and third generations of quarks and leptons have identical quantum numbers. There is no right-handed neutrino in the Standard Model because it cannot interact with any of the gauge bosons.

\begin{tabular}{|l|l|c|c|c|c|}
\hline & Fermion & $T$ & $T^{3}$ & $Q$ & $Y$ \\
\hline Leptons & $\nu_{e}$ & $\frac{1}{2}$ & $\frac{1}{2}$ & 0 & -1 \\
& $e_{L}^{-}$ & $\frac{1}{2}$ & $-\frac{1}{2}$ & -1 & -1 \\
& $e_{R}^{-}$ & 0 & 0 & -1 & -2 \\
\hline Quarks & $u_{L}$ & $\frac{1}{2}$ & $\frac{1}{2}$ & $\frac{2}{3}$ & $\frac{1}{3}$ \\
& $d_{L}$ & $\frac{1}{2}$ & $-\frac{1}{2}$ & $-\frac{1}{3}$ & $\frac{1}{3}$ \\
& $u_{R}$ & 0 & 0 & $\frac{2}{3}$ & $\frac{4}{3}$ \\
& $d_{R}$ & 0 & 0 & $-\frac{1}{3}$ & $-\frac{2}{3}$ \\
\hline
\end{tabular}

where $Q$ is charge and $T^{3}$ is the third component of isospin (see Table 1.2 for a listing of $T^{3}$ for the fermions in the Standard Model). The corresponding current follows immediately:

$$
J_{\mu}^{Y}=2\left(J_{\mu}^{\epsilon m}-J_{\mu}^{3}\right)
$$

The gauge group generated by $Y$ is often referred to as $U(1)_{Y}$. The gauge field introduced to maintain local $U(1)_{Y}$ invariance is labeled $B_{\mu}$ and carries an independent coupling constant $g^{\prime}$. The interaction term in the Lagrangian becomes

$$
\mathcal{L}_{\text {int }}^{E W}=-i g\left(J^{i}\right)^{\mu} W_{\mu}^{i}-i \frac{g^{\prime}}{2}\left(J^{Y}\right)^{\mu} B_{\mu}
$$

The fields

$$
W_{\mu}^{ \pm}=\sqrt{\frac{1}{2}}\left(W_{\mu}^{1} \mp i W_{\mu}^{2}\right)
$$

describe the physical weak charged currents and $W_{\mu}^{3}$ and $B_{\mu}$ are neutral fields. The linear combination of neutral fields necessary to represent the physical neutral gauge bosons $\gamma$ and $Z^{0}$ is not specified by the theory, but may be parameterized by the Weinberg angle $\theta_{W}$ :

$$
A_{\mu}=B_{\mu} \cos \theta_{W}+W_{\mu}^{3} \sin \theta_{W}
$$

and

$$
Z_{\mu}=-B_{\mu} \sin \theta_{W}+W_{\mu}^{3} \cos \theta_{W}
$$


Since the coupling of the electromagnetic current $J_{\mu}$ to the photon field has strength $e$, we may identify the couplings

$$
g \sin \theta_{W}=g^{\prime} \cos \theta_{W}=e,
$$

which has the immediate consequence

$$
\tan \theta_{W}=\frac{g^{\prime}}{g}
$$

At this point, the only parameters of the electroweak interaction that need to be determined from experiment are the values of the coupling constant $e$ and the Weinberg angle $\theta_{W}$.

\subsubsection{Spontaneous Symmetry Breaking and Gauge Boson Masses}

In the previous section we have ignored the fact that some of the gauge bosons indeed have mass. It is this mass that makes the weak interactions "weak." After all, the coupling constants are closely related to the QED coupling, yet the forces mediate interactions that happen much more slowly than electromagnetic ones. The weakness of the weak force is just a manifestation of the denominator of the propagator of the $W^{ \pm}$and the $Z^{0}$. For low- $Q^{2}$ interactions, the energy dependence of the propagator is -dwarfed by the masses of the weak bosons $(\sim 90 \mathrm{GeV})$, that it can be safely approximated by a constant that can be combined with the coupling constant to form an effective strength at low energies.

Introduction of mass into the electroweak model has to be done with some delicacy, because the $S U(2)_{L} \times U(1)_{Y}$ symmetry prohibits mass terms of the form $M_{W}^{2} W_{\mu} W^{-\mu}$ just as the QED $U(1)$ symmetry does. The goal is to introduce an interaction whose Lagrangian preserves the $S U(2)_{L} \times U(1)_{Y}$ symmetry, but whose ground state breaks it. It is not necessary to break the entire symmetry group, as the photon is to remain massless - the $U(1)_{E M}$ subgroup of the theory is to remain unbroken.

The solution involves hypothesizing four additional scalar fields $\phi_{i}$ and adding to the Lagrangian of the Standard Model the term

$$
\mathcal{L}_{\text {Higgs }}=\left|\left(i \partial_{\mu}-g \sigma^{i} W_{\mu}^{i}-g^{\prime} \frac{Y}{2} B_{\mu}\right) \phi\right|^{2}-V(\phi)
$$

The simplest choice of fields $\phi$ that preserve the gauge invariance of $\mathcal{L}_{\text {Higgs }}$ is an isodoublet with weak hypercharge $Y=1$, a choice originally made by Weinberg:

$$
\phi=\left(\begin{array}{c}
\left(\phi_{1}+i \phi_{2}\right) / \sqrt{2} \\
\left(\phi_{3}+i \phi_{4}\right) / \sqrt{2}
\end{array}\right)
$$

The symmetry is broken by a judicious choice of the potential function

$$
V(\phi)=\mu^{2} \phi^{\dagger} \phi+\frac{\lambda}{2}\left(\phi^{\dagger} \phi\right)^{2}
$$

chosen with $\mu^{2}<0$ and $\lambda>0$. This potential is minimized when

$$
\phi^{\dagger} \phi=-\mu^{2} / \lambda .
$$

This set of $\phi$ that minimizes $V$ is invariant under $S U(2)$ transformations, although when the system settles into a ground state, it only chooses one point among the possible ones. Without loss of generality we may set $\phi_{1}, \phi_{2}$, and $\phi_{4}$ to zero, then

$$
\phi_{3}^{2}=-\frac{\mu^{2}}{\lambda} \equiv v^{2}
$$

The vacuum $\phi$ in this case is

$$
\phi_{0}=\sqrt{\frac{1}{2}}\left(\begin{array}{l}
0 \\
v
\end{array}\right) .
$$

Because there are four degrees of freedom of $\phi$ and only one constraint which specifies the minimum, the minimizing manifold is three-dimensional. Since $\phi$ may fluctuate within this manifold without an energy penalty, these fluctuations correspond to three massless Nambu-Goldstone bosons [26]. These additional degrees of freedom are actually just overcounting the gauge degrees of freedom, and do not appear in the standard Lagrangian. The remaining degree of freedom corresponds to a physical scalar Higgs field $h$ with a particle whose mass is $-\lambda v^{2} / 2$. The relevant mass-generating term for 
the gauge bosons in Equation 1.24, expressed in the basis of physical gauge bosons, then is

$$
\begin{aligned}
& \left|\left(-i g \frac{1}{2} \sigma^{i} W_{\mu}^{i}-i \frac{g^{\prime}}{2} B_{\mu}\right) \phi\right|^{2}= \\
& \quad\left(\frac{1}{2} v g\right)^{2} W_{\mu}^{+} W^{-\mu}+\frac{1}{8} v^{2}\left[g W_{\mu}^{3}-g^{\prime} B_{\mu}\right]^{2}+0\left[g^{\prime} W_{\mu}^{3}+g B_{\mu}\right]^{2} .
\end{aligned}
$$

From this relation, the coefficients of the terms quadratic in the fields yield the masses of the vector bosons. The mass of the charged $W$ is the simplest, with

$$
M_{W \pm}=\frac{1}{2} v g
$$

Using $1.20,1.21$, and 1.23 , we obtain

$$
A_{\mu}=\frac{g^{\prime} W_{\mu}^{3}+g B_{\mu}}{\sqrt{g^{2}+g^{\prime 2}}}
$$

for the photon, and so its mass coefficient is zero from Equation 1.30, and

$$
Z_{\mu}=\frac{g W_{\mu}^{3}-g^{\prime} B_{\mu}}{\sqrt{g^{2}+g^{\prime 2}}},
$$

yielding the $Z^{0}$ mass, also read off from Equation 1.30

$$
M_{Z}=\frac{1}{2} v \sqrt{g^{2}+g^{\prime 2}} \text {. }
$$

Combining 1.34 with 1.31 yields the relation

$$
\frac{M_{W}}{M_{Z}}=\rho \cos \theta_{W}
$$

where $\rho=1$ until radiative corrections are applied.

The parameters of the electroweak sector of the Standard model number three. They are $e, \sin ^{2} \theta_{W}$, and $v$, the vacuum expectation value of the Higgs. In terms of observables that can be measured with precision, three necessary parameters are $\alpha_{E M}, M_{Z}$, and $G_{\mu}$. With these three parameters, Standard Model predictions at tree level (modulo effects like phase space which depend on fermion masses). At one loop. the masses of fermions and the Higgs mass enter, and non-standard phenomena may have effects at energies achievable with current accelerators.

\subsection{The Strong Interaction}

An integral portion of the standard picture of elementary particle interactions is the model of the strong interaction. Various detailed treatments are available in the literature [27] [22]. The strong interaction has many features which differentiate it from the electroweak interaction, although both can be formulated as local gauge theories. These differences include

- Range. The strong interaction has a very short range, while the electromagnetic portion of the electroweak interaction has an infinite range.

- Asymptotic Freedom. The strength of the strong interaction decreases with increasing $Q^{2}$ of the interaction. This feature was observed first in deep-inelastic $e-p$ scattering.

- Confinement. The partons within hadrons cannot be isolated. No observable particle has a bare color charge.

- Symmetry. The strong interaction obeys flavor symmetry and also conserves parity.

- Hadron Structure. Quarks are bound within hadrons which contain either three quarks, three antiquarks, or a quark and an antiquark.

The quark model originally contained a paradox regarding the structure of hadrons. Some baryons, it seemed, violated the spin-statistics theorem in that three quarks in them seemed to occupy the same quantum state. These hadxons include the $\Delta^{++}$and the $\Omega^{-}$. The flavor assignments for the quarks are all the same, for both of these baryons, and each quark only has two spin states available to it, so there is no assignment of spins that can preserve the Pauli exclusion principle. Furthermore, the spin of the $\Delta^{++}$was found to be $3 / 2$, indicating that all three quarks shared the same spin state. Another degree of freedom with at least three distinct values needed to be introduced to preserve the statistics.

The model which grew out of that necessity and which best describes the strong interactions is referred to as "Quantum Chromodynamics," and is formulated as a 
non-Abelian Yang-Mills gauge theory with $S U(3)$ as its symmetry group; each quark transforms as a triplet (three values of the color charge). The local gauge symmetry for QCD for a quark of flavor $k=u, d, s, c, b$, or $t$ is

$$
q_{k} \rightarrow e^{i g \lambda_{a} \Lambda_{a}} q_{k},
$$

where the $\lambda_{a}$ are the eight generator matrices of $S U(3)$, commonly known as GellMann matrices, and $\Lambda_{\alpha}$ are functions of space and time. To construct a covariant derivative that is gauge invariant, eight fields $A_{\mu}^{a}$, corresponding to eight bi-colored gluons, must be introduced in the same way as they were for the electroweak interaction:

$$
D_{\mu} q_{k}=\left(\partial_{\mu}-i g A_{\mu}\right) q_{k}
$$

with

$$
A_{\mu}=\sum_{a=1}^{8} A_{\mu}^{a} \lambda^{a} / 2
$$

The coupling constant $g$ is a single parameter left to be determined experimentally: The Lagrangian can then be expressed as

$$
\mathcal{L}_{Q C D}=-\frac{1}{2} \operatorname{tr} G_{\mu \nu} G^{\mu \nu}+\sum_{k=1}^{n_{\text {flavors }}} \bar{q}_{k}\left(i \gamma^{\mu} D_{\mu}-m_{k}\right) q_{k}
$$

with the field-strength tensor $G_{\mu \nu}$ defined similarly to that of QED, with a nonAbelian piece added:

$$
G_{\mu \nu}=\partial_{\mu} A_{\nu}-\partial_{\nu} A_{\mu}-i g\left[A_{\mu}, A_{\nu}\right] .
$$

In this manner, QCD has been referred to as "eight copies of QED," although its non-Abelian nature lends it some rather unique properties.

The non-Abelian terms in the QCD Lagrangian give rise to interactions between gluons, the gauge bosons of QCD. This is a manifestation of the property of gluons in that not only do they couple to objects with color charge, they themselves carry color charge. The new diagrams introduced into the theory are a triple-gluon vertex, as well as a four-gluon vertex, shown in Figure 1.1

In contrast to the electroweak gauge boson sector, there is no evidence for gluon mass, and so the mechanism of spontaneous symmetry breaking is not necessary.

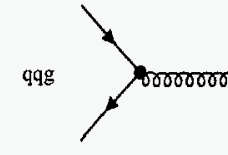

ggg

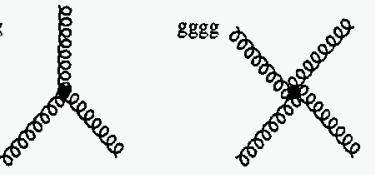

Figure 1.1: Vertex diagrams in QCD

Further evidence for QCD comes from the energy-dependent behavior of

$$
R_{\text {had }}=\frac{\Gamma\left(e^{+} e^{-} \rightarrow \text { hadrons }\right)}{\Gamma\left(e^{+} e^{-} \rightarrow \mu^{+} \mu^{-}\right)} \text {. }
$$

This function shows distinct energy thresholds, marked by resonances at which $q \bar{q}$ pairs are produced that form vector mesons. Above these thresholds, $R_{\text {had }}$ assumes a higher value because of the availability of more hadronic final states. The rate for $e^{+} e^{-} \rightarrow \mu^{+} \mu^{-}$is readily calculable from QED (and the electroweak interaction at higher energies), and so jumps in the value of $R_{\text {had }}$ above thresholds quantitatively measure the numbers of new available final states. It is observed that the changes in $R_{\text {had }}$ correspond to three times that which would be naïvely predicted if the quark color degrees of freedom were ignored.

This model of strong interactions has the requisite ingredients to satisfy the properties listed at the beginning of this section. In particular, the non-Abelian selfinteraction of the gluons provides QCD with the ability to explain asymptotic freedom [28][27]. In contrast to the Abelian QCD case, in which the vacuum polarizes to screen charges at large distances, the QCD vacuum actually anti-screens charges, and its coupling becomes stronger at large distances and lower energy scales. This feature 
of QCD is the reason many believe it has the ability to model quark confinement, although a rigorous demonstration has not been produced yet.

If a quark within a hadron is struck, say, with an impinging photon, it will pull away from the other quark(s) in the hadron. Because the strength of the color interaction increases with distance, energy stored in the field of gluons between the quarks builds as the struck quark moves away. At some point, it becomes energeti. cally favorable to produce a $q \bar{q}$ pair from the vacuum. The colors chosen for the new $q \bar{q}$ pair must be chosen so that the newly formed hadrons are color singlets. It is this process, called fragmentation, which gives the hadronic decays of the $Z^{0}$ their characteristic structure in the SLD; quarks are not observed in the final state, even though very high-energy, back-to-back quarks are produced by the decay. Instead, a large number of color-singlet hadrons, traveling in largely the same directions as the original quarks, appears in the detector. This process of hadron formation cannot be predicted perturbatively, although it can be modeled with computer simulation. A recursive algorithm was proposed by Feynman and Field [29], and its latest implementation for simulations of $e^{+} e^{-}$collisions is the JETSET 7.4 Monte Carlo [30][31]. used in this thesis.

The property of asymptotic freedom also plays a role in the structure of $Z^{0}$ decars. Very high- $Q^{2}$ processes may be modeled perturbatively because the strong coupling constant $\alpha_{s}$ has a relatively small value at high energy. The value of $\alpha_{s}$ at the $Z^{0}$ energies is $\sim 0.12$, with ambiguities arising from the different techniques for measuring it and their interpretations. Perturbation theory for the strong interactions is not quite as safe as that for $\mathrm{QED}$, owing to its larger coupling constant - it is not clear whether some perturbative series converge at all. Modeling of non-perturbative effects may be accomplished with the aid of lattice QCD computer simulations [32].

Nonetheless, perturbative QCD radiation plays a large role in hadronic $Z^{0}$ decay. Visible in many such decays is a third "jet" of final-state hadrons, corresponding to a hard gluon radiated by the quarks [33]. Because the gluon is a colored object. which may not escape to large distances, it too must fragment into hadrons. The rate at which this process happens is a measure of $\alpha_{s}$, although the energy scale of this interaction is somewhat ambiguous [34][35].
The combined effect of hard gluon radiation and the fragmentation process removes energy from the original quark emitted by the $Z^{0}$. For the case of $Z^{0} \rightarrow u \bar{u}$ or $Z^{0} \rightarrow d \bar{d}$, the many quarks pulled from the vacuum are indistinguishable from the ones into which the $Z^{0}$ decayed. But in the case of a heavy flavor decay of the $Z^{\circ}$, there is only one heavy-flavor hadron in each hemisphere, and the fragmentation function, describing the energy of that hadron as a fraction of half of $\sqrt{s}$, is well defined. For the light-flavor decays of the $Z^{0}$, the energy-loss fraction is an important ingredient in the recursive algorithm describing the hadronization process.

The fragmentation function describing the momentum of the fastest hadron in light-quark events is well parameterized by the "Lund symmetric function:" $[30$

$$
f(z) \propto z^{-1}\left(1-z^{2}\right)^{a} \epsilon^{-b m_{\perp}^{2} / z}
$$

where $z=2 E$ (hadron $) / \sqrt{s}, m_{\perp}$ is the "transverse mass"

$$
m_{\perp}^{2}=E^{2}-p_{\|}^{2}
$$

and $a$ and $b$ are tunable parameters. The fragmentation function iteratively describes hadron formation for lower-energy hadrons in the parton shower. The parameters a and $b$ typically have values of 0.18 and $0.34 \mathrm{GeV}^{-2}[36]$ so the momentum distributions of final-state particles best match available data. It is called a "symmetric" function because it describes a breaking string as viewed either from either end. This model has been tremendously successful at a wide range of energies. The values of $a$ and $b$ were originally tuned at PETRA energies and the model reproduces the observed hadron spectrum at LEP energies with very little further adjustment.

The model above does not describe heavy flavor production very well; the actual fragmentation function for $c$ and $b$ quarks is much harder than that for the light flavors. The reason the heavy hadron carries a larger fraction of the available energy is described in [37]. In short, the additional hadrons formed in the fragmentation process are produced with a speed (or boost, $\gamma$ ) that is less than the speed (or $\gamma$ ) of the leading heavy quark. Because the $\gamma$ of the heavy quark before the fragmentation process scales as $1 / m_{Q}$, the energy fractions the extra fragmentation tracks receive is $\sim 1 \mathrm{GeV} / m_{Q}$. 


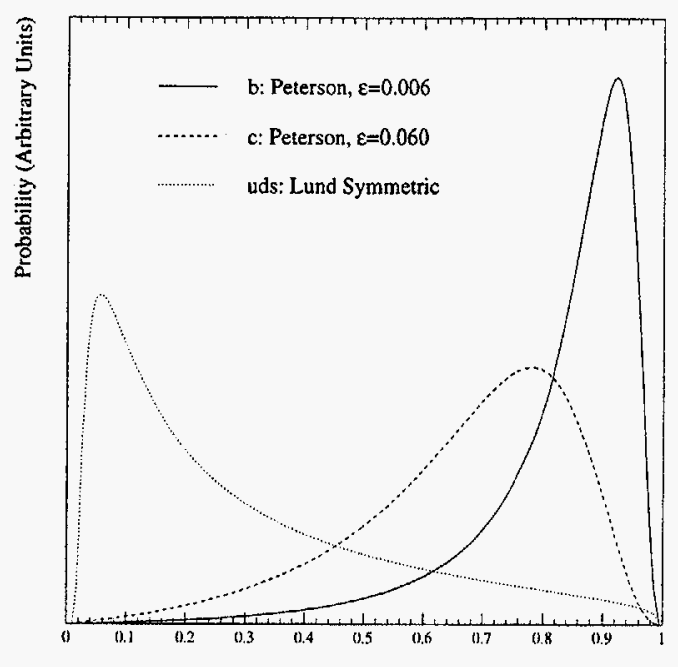

$\mathrm{z}$

Figure 1.2: The fragmentation functions for $u d s, c$, and $b$. The Lund symmetric function, described in the text, depends strongly on the value of $m_{\perp}$, which is chosen on each splitting. Shown here is the Lund symmetric function with $m_{1}=300 \mathrm{MeV} / \mathrm{c}^{2}$

The fragmentation function commonly used to model $Z^{0} \rightarrow b \bar{b}$ and $Z^{0} \rightarrow c \bar{c}$ is the Peterson function:

$$
f(z) \propto \frac{1}{z\left(1-(1 / z)-\epsilon_{Q} /(1-z)\right)^{2}},
$$

with the parameter $\epsilon_{Q}$ chosen to be 0.060 for $c$ quarks, and 0.006 for $b$ quarks. These fragmentation distributions are shown in Figure 1.2. The stiffness of the $b$ fragmentation function is one of the features that allows a momentum-weighted charge measurement of $A_{b}$ to be effective, because weighting the tracks' charges with their momenta de-emphasizes the role of fragmentation tracks.

\subsection{Tree-Level Asymmetries at the $Z^{0}$}

Observable asymmetries on the $Z^{0}$ resonance depend almost entirely on the couplings of fermions to the $Z^{0}$, but since there is a small contribution from $s$-channel $\gamma$ exchange, it will be included here. The couplings of the fermions to the $Z^{0}$ and the $\gamma$ are summarized in Table 1.3 [38]. The notation is described below.

The neutral current coupling of a fermion $f$ to the $Z^{0}$ has vector and axial-vector components parameterized by $v^{Z f}$ and $a^{Z f}$ :

$$
J^{Z f} \propto \bar{f}\left(v^{Z f}+a^{Z f} \gamma_{5}\right) f
$$

The coupling of the same fermion $f$ to the photon is purely a vector coupling and its strength is proportional to the charge of the fermion $Q_{f}$. Because the spin projection operators have the forms

$$
\begin{aligned}
& P_{L}=\left(1-\gamma_{5}\right) / 2, \quad \text { and } \\
& P_{R}=\left(1+\gamma_{5}\right) / 2,
\end{aligned}
$$

the left-handed and right-handed couplings of the fermion $f$ to the $Z^{0}$ may be defined to be

$$
\begin{aligned}
c_{L}^{Z f} & =\left(v^{Z f}+a^{Z f}\right) / 2, \quad \text { and } \\
c_{R}^{Z f} & =\left(v^{Z f}-a^{Z f}\right) / 2 .
\end{aligned}
$$

The energy dependence of the observable asymmetries to be described has its origin in the combination of two gauge bosons, the $\gamma$ and the $Z^{0}$, in the propagator. When adding the graphs of Figure 1.3, the matrix element for $e^{+} e^{-} \rightarrow f \bar{f}$ is

$$
M_{i j}=\left(\frac{e}{\sin \theta_{W} \cos \theta_{W}}\right)^{2} \frac{-i}{s-m_{Z}^{2}+i \Gamma_{Z} m_{Z}} c_{i}^{Z \epsilon} c_{j}^{Z f}+e^{2} \frac{-i}{s} Q_{e} Q_{f}
$$

where $i, j=L, R$ are the helicity indices for the initial state electron and final-state fermion respectively.

In what follows, only the angular and initial- and final-state helicity dependences of the differential cross-sections will be retained, and the energy dependence of the 
Table 1.3: Chiral couplings of the standard fermions to the $\gamma$ and $Z^{0}$. The couplings to the $\gamma$ are spin-independent and depend only on the charge $Q_{f}$.

\begin{tabular}{|l|c|cccc|}
\hline & & \multicolumn{4}{|c|}{$Z^{0}$ coupling } \\
fermion & $Q_{f}$ & $v^{Z f}$ & $a^{Z f}$ & $c_{R}^{Z f}$ & $c_{L}^{Z f}$ \\
\hline$e, \mu, \tau$ & -1 & $-\frac{1}{2}+2 \sin ^{2} \theta_{W}$ & $-\frac{1}{2}$ & $\sin ^{2} \theta_{W}$ & $-\frac{1}{2}+\sin ^{2} \theta_{W}$ \\
$\nu_{e}, \nu_{\mu}, \nu_{\tau}$ & 0 & $\frac{1}{2}$ & $\frac{1}{2}$ & 0 & $\frac{1}{2}$ \\
$u, c, t$ & $\frac{2}{3}$ & $\frac{1}{2}-\frac{4}{3} \sin ^{2} \theta_{W}$ & $\frac{1}{2}$ & $-\frac{2}{3} \sin ^{2} \theta_{W}$ & $\frac{1}{2}-\frac{2}{3} \sin ^{2} \theta_{W}$ \\
$d, s, b$ & $-\frac{1}{3}$ & $-\frac{1}{2}+\frac{2}{3} \sin ^{2} \theta_{W}$ & $-\frac{1}{2}$ & $\frac{1}{3} \sin ^{2} \theta_{W}$ & $-\frac{1}{2}+\frac{1}{3} \sin ^{2} \theta_{W}$ \\
\hline
\end{tabular}

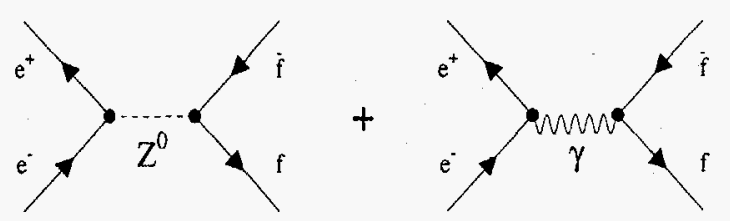

Figure 1.3: Tree-level electroweak contributions to $e^{+} e^{-} \rightarrow f \bar{f}$ in the Standard Model.

total cross section will be factored out. In addition, the final state $\epsilon^{+} \epsilon^{-}$will be omitted because there is an additional $t$-channel $\gamma$-exchange diagram which contributes significantly, and even diverges at small scattering angles.

Equation 1.48 provides a definition of the left- and right-handed couplings to the combined electroweak current. It is convenient in what follows to express the product $c_{i}^{e} c_{j}^{f}$ with the same helicity indices $i, j$ as before:

$$
c_{i}^{e} c_{j}^{f}=c_{i}^{Z e} c_{j}^{Z f}+Q_{e} Q_{j} \Lambda
$$

where

$$
\Lambda(s)=\frac{s-m_{Z}^{2}+i \Gamma_{Z} m_{Z}}{s} \sin ^{2} \theta_{W} \cos ^{2} \theta_{W}
$$

The square of the combined couplings appears in the final cross sections, so a more useful combination is

$$
\left|c_{i}^{e} c_{j}^{f}\right|^{2}=\left(c_{i}^{Z e} c_{j}^{Z f}\right)+2 c_{i}^{Z e} c_{j}^{Z f} Q_{e} Q_{f} \operatorname{Re}\{\Lambda(s)\}+Q_{e}^{2} Q_{f}^{2}|\Lambda(s)|^{2} .
$$

The first term of this relation is the pure $Z^{0}$ coupling; the last is a $\gamma$-exchange term, and the middle term is the $\gamma-Z$ interference term. If $s=m_{Z}^{2}$, this interference term vanishes, reducing the sensitivity of asymmetry measurements to additional neutral gauge bosons, including the photon.

If $\theta$ is defined to be the angle between the incoming $e^{-}$beam and the outgoing fermion $f$, then

$$
\begin{aligned}
& \frac{d \sigma_{R R}}{d \Omega} \propto\left(c_{R}^{e} c_{R}^{f}\right)^{2}\left(1+\cos ^{2} \theta+2 \cos \theta\right), \\
& \frac{d \sigma_{L L}}{d \Omega} \propto\left(c_{L}^{e} c_{L}^{f}\right)^{2}\left(1+\cos ^{2} \theta+2 \cos \theta\right), \\
& \frac{d \sigma_{L R}}{d \Omega} \propto\left(c_{L}^{e} c_{R}^{f}\right)^{2}\left(1+\cos ^{2} \theta-2 \cos \theta\right), \\
& \frac{d \sigma_{R L}}{d \Omega} \propto\left(c_{R}^{e} c_{L}^{f}\right)^{2}\left(1+\cos ^{2} \theta-2 \cos \theta\right) .
\end{aligned}
$$

and

The helicity of the position is not specified because it is fixed by the helicity of the incoming electron beam. A left-handed electron will only couple with a right-handed positron at the $Z e e$ vertex owing to helicity conservation at high energies*. The above relations convey in their functional form only the conservation of angular momentum: the actual parity violation comes about by the differing values of $c_{R}^{f}$ and $c_{L}^{f}$. This can easily be summarized in that if a $Z^{0}$ is produced with a right-handed electron beam traveling in the $+\hat{z}$ direction, then the expectation value of the spin of the $Z^{0}$ is unity in the $+\hat{z}$ direction. Since the $Z^{0}$ prefers to couple to left-handed fermions and right-handed antifermions, then the antifermion will be sent forwards (along the direction of travel of the $e^{-}$) preferentially, and the fermion backwards, in order to conserve angular momentum.

The polarization of the outgoing fermion cannot be ascertained from experimental observables, except in the important case of the $\tau$, so the polarized cross-sections must "It is belicity conservation that suppresses direct production of scalars at high-energy $\epsilon^{+} \epsilon^{-}$ machines, by a factor of $m_{e}^{2} / s$. 
Table 1.4: Values of $A_{f}$ for $\sin ^{2} \theta_{W}=0.23$, calculated with radiative corrections in reference [39], and their sensitivity to $\sin ^{2} \theta_{W}$.

\begin{tabular}{|ccc|}
\hline Fermion type & $A_{f}$ & $\frac{\partial A_{f}}{\partial \sin ^{2} \theta_{W}}$ \\
\hline$e, \mu, \tau$ & 0.16 & -7.85 \\
$u, c, t$ & 0.67 & -3.45 \\
$d, s, b$ & 0.94 & -0.63 \\
\hline
\end{tabular}

be summed over final-state fermion polarizations to obtain

$$
\frac{d \sigma_{R}}{d \Omega} \propto\left[\left(c_{R}^{f 2}+c_{L}^{\prime 2}\right)\left(1+\cos ^{2} \theta\right)+2\left(c_{R}^{f 2}-c_{L}^{f 2}\right) \cos \theta\right]
$$

and

$$
\frac{d \sigma_{L}}{d \Omega} \propto\left[\left(c_{R}^{f 2}+c_{L}^{f 2}\right)\left(1+\cos ^{2} \theta\right)-2\left(c_{R}^{f 2}-c_{L}^{f 2}\right) \cos \theta\right],
$$

where the helicity index on $\sigma$ refers now only to the incident electron beam. As a further simplification, we may combine the fermion couplings into asymmetry param eters

$$
A_{f} \equiv \frac{2 v_{f} a_{f}}{v_{f}^{2}+a_{f}^{2}}=\frac{c_{L}^{f}-c_{R}^{f}}{c_{L}^{f}+c_{R}^{f}} .
$$

Substituting in the vector and axial vector couplings of the fermions to the $Z^{0}$ in terms of $\sin ^{2} \theta_{W}$, before radiative corrections,

$$
A_{f}^{S M, B \circ r n}=\frac{1+\left|Q_{f}\right|^{2} \sin ^{2} \theta_{W}}{1+\left(1+\left|Q_{f}\right|^{2} \sin ^{2} \theta_{W}\right)^{2}} .
$$

The mass of the final-state fermion couples its $L$ and $R$ states and gives it a finite probability to fip its spin. The modification to the $A_{f}$ parameter is $[40]^{\dagger}$

$$
A_{f}=\frac{2 \beta v_{f} a_{f}}{\frac{3-\beta^{2}}{2} v_{f}^{2}+\beta^{2} a_{f}^{2}},
$$

${ }^{\dagger}$ In reference [40], both the formulas for the $\beta$-dependent $A_{f}$ and $\beta$ have typographical errors The correct formulas are presented here. where $\beta^{2}=1-4 \frac{m_{f}^{2}}{m_{2}^{2}}$ is the speed of the outgoing fermion. For the $b$ quark, this increases the expected asymmetry by $\sim 0.02 \%$ and will be accounted for in this thesis.

Using the parameters $A_{f}$, omitting the dependence on energy, and creating a linear combination of $L$ and $R$ polarization states to form a partially unpolarized initial state, the angular dependence of the differential cross section can be expressed as

$$
\frac{d \sigma^{f}\left(P_{e}\right)}{d \cos \theta} \propto\left(1-A_{e} P_{e}\right)\left(1+\cos ^{2} \theta\right)+2\left(A_{e}-P_{e}\right) A_{f} \cos \theta
$$

where $P_{e}$ is the polarization of the incoming electron beam. With this formalism we may then parameterize the tree-level Standard Model predictions of observable asymmetries $A_{L R}, A_{F B}^{f}, A_{F B}^{\tau p o l}$, and $\tilde{A}_{F B}^{f}$.

\subsubsection{The Left-Right Production Asymmetry $A_{L R}$}

One of the simplest and most powerful asymmetries that can be formed with an experiment with a polarized electron beam is the left-right asymmetry $A_{L R}$ which directly measures the asymmetry in the chiral couplings of electrons to the $Z^{\circ}$. It is measured by producing equal amounts of luminosity using a left-handed and righthanded electron beam on unpolarized positrons. It then remains to count the $f \bar{f}$ final states (rejecting the $e^{+} e^{-}$final state) for both initial helicities and form the asymmetry

$$
A_{L R}^{r a w}=\frac{N_{L}-N_{R}}{N_{L}+N_{R}}=P_{e} A_{e} .
$$

SLD's measurements of $A_{L R}$ are described in References [41] and [42]

One of the main virtues of this asymmetry is that it does not require the isolation of any particular final state, except the experimentally distinct $e^{+} \epsilon^{-}$state. Another benefit of this measurement is that it is very insensitive to the details of the detector. As long as its detection efficiency for fermions is the same as that for antifermions at each value of $\cos \theta$, efficiency effects cancel in the ratio. Because the $Z^{0}$ decays into a back-to-back fermion-antifermion pair, this detection efficiency can be further safeguarded by building a detector that is symmetric about its midplane perpendicular to the beam axis. Its third virtue is that it is a very sensitive measurement of $\sin ^{2} \theta w$. Its dependence can be obtained from Equation 1.59 and is listed in Table 1.4. Given 
SLC's electron polarization of $77 \%$ in 1994 , this single asymmetry provides the most powerful technique of measuring $\sin ^{2} \theta_{W}$ at present.

The $\sin ^{2} \theta_{W}$ that is measured by $A_{L R}$ is a physical parameter, and so necessarily: incorporates effects of propagator and vertex radiative corrections, to be described in Section 1.5. The measurement, however, is corrected for the effects of initial-state radiation, which may be calculated from QED. The measurement yields $\sin ^{2} \theta_{W}^{e f f}$, the "effective" weak mixing angle parameter [43], which parameterizes the remaining radiative corrections. Vertex corrections at the final vertex do not affect the couplings at the initial vertex significantly, unless the energy is far from the $Z^{0}$ pole and $\gamma$ exchange becomes significant. The most important radiative corrections are the vacuum polarization diagrams in the gauge boson propagator. These lend to $A_{L R}$ sensitivity to $m_{\text {top }}$ as well as $m_{\text {Higgs }}$. With the recent measurements of $m_{\text {top }}$ from CDF [18] and D0 [19], this measurement may provide the best knowledge of what mass the Higgs boson is expected to have.

The dependence of $A_{L R}$ on the colliding energy of the beams is shown in Figure 1.4. highlighting the need to correct for off-pole measurements.

\subsubsection{Unpolarized Forward-Backward Asymmetries $A_{F B}^{f}$}

In the absence of a polarized electron beam, electroweak asymmetry measurements must rely on other parity-violating asymmetries. One of these approaches is to compare the number of fermions of a particular species which travel forwards in the detector (along the direction of the electron beam) to those that travel backwards. Because the $Z^{0}$ couples preferentially to the left-handed component of the unpolarized electron beam, the expectation value of its spin along the $\hat{z}$ axis does not vanish, and there is an asymmetry in the forward-backward distribution of fermions in the final state, $A_{F B}^{f}$. This asymmetry relies on parity violation at both the production and decay vertices, so its value is proportional to the product of initial- and finalstate coupling asymmetries:

$$
A_{F B}^{f}\left(P_{\mathrm{e}}=0\right)=\frac{\sigma_{F}^{f}-\sigma_{B}^{f}}{\sigma_{F}^{f}-\sigma_{B}^{f}}=\frac{3}{4} A_{e} A_{f},
$$

where

and

$$
\sigma_{B}=\int_{-1}^{0} \frac{d \sigma}{d \cos \theta} d \cos \theta
$$

$$
\sigma_{F}=\int_{0}^{1} \frac{d \sigma}{d \cos \theta} d \cos \theta
$$

The differential cross-sections $\sigma_{F, B}$ are averaged over initial state polarizations and summed over final state polarizations, as at an unpolarized experiment, there is no control over either one. Care must be taken, however, that the initial state is genuinely unpolarized, as the effect on the cross section of any residual polarization is quite dramatic.

One feature of the definition of $A_{F B}^{f}$ is that it involves an asymmetry of cross sections integrated over $\cos \theta$, and particle detectors invariably have a loss of acceptance at large values of $|\cos \theta|$ owing to their solenoidal nature and the necessity of having an aperture for the beams. A more ideal quantity to form involves finding the forward-backward asymmetry as a function of $\mid \cos \theta$ and then fitting the function

$$
A_{F B}^{f}(|\cos \theta|)=\frac{\sigma^{f}(\cos \theta)-\sigma^{f}(-\cos \theta)}{\sigma^{f}(\cos \theta)+\sigma^{f}(-\cos \theta)}=A_{e} A_{f} \frac{2|\cos \theta|}{1+\cos ^{2} \theta}
$$

to it. With this technique, the detector acceptance function cancels in the numerator and denominator of the formed asymmetry, if $\mathrm{jt}$ is binned finely enough.

For the lepton final states $\mu^{+} \mu^{-}$and $\tau^{+} \tau^{-}$this asymmetry measures the products $A_{e} A_{\mu}$ and $A_{e} A_{\tau}$. Assuming that the lepton coupling asymmetries are identical, then the quantity $A_{e}^{2}$ is measured, with its strong dependence on $\sin ^{2} \theta_{W}$ given in Table 1.4 .

To improve the statistical power of the measurement of $\sin ^{2} \theta_{W}$ at LEP, $A_{F B}^{b}$ is measured, largely because the value of $A_{b}$ is large in the Standard Model, 0.94, and because pure and efficient samples of $Z^{0} \rightarrow b \bar{b}$ may be obtained by either tagging high- $P_{T}$ leptons from $B$ decay, or by using precision vertex detectors to identify longlive particles. Nonetheless, it is still the combination $\frac{3}{4} A_{e} A_{b}$ which is measured, with most of the dependence on $\sin ^{2} \theta_{W}$ coming from $A_{e}$. The Standard Model must be invoked to provide a prediction of $A_{b}$ in order to extract $\sin ^{2} \theta_{W}$.

The energy dependence of $A_{F B}^{b}$ is shown in Figure 1.4. The LEP accelerator performs energy scans, so the energy dependence of this asymmetry near the $Z^{0}$ pole can be measured by each of the LEP experiments. 


\subsubsection{Forward-Backward Tau Polarization Asymmetry $A_{F B}^{P(\tau)}$}

An $e^{+} e^{-}$experiment with unpolarized beams is incapable of measuring $A_{L R}$ directly, and the $A_{F B}^{f}$ measurements are sensitive to a product of asymmetries. If there is new physics at a vertex or just a poorly modeled radiative correction, then the extraction of $A_{e}$ from the $A_{F B}^{f}$ will be incorrect. The asymmetry of $e^{+} e^{-} \rightarrow e^{+} e^{-}$would reduce the assumptions on the asymmetries, but its value is modified by $t$-channel exchange diagrams.

Nonetheless, an independent measurement of $A_{e}$ can be made at an unpolarized experiment, provided that the data sample is large. The technique used is to measure the final-state polarization of $\tau$ leptons from $Z^{0}$ decay. The taus themselves have asymmetric couplings to the $Z^{0}$, and exhibit a forward-backward asymmetry. The polarization of the taus as a function of polar angle depends only on the expectation value of the spin of the $Z^{0}$, and is a consequence of angular momentum conservation, as expressed in equations 1.52-1.55. Using these relations, one may derive the expectation value of the final-state fermion polarization,

$$
P_{r}(\theta)=\frac{-A_{\tau}\left(1+\cos ^{2} \theta\right)+2 F\left(P_{e}\right) \cos \theta}{1+\cos ^{2} \theta-2 A_{\tau} F\left(P_{e}\right) \cos \theta}
$$

where

$$
F\left(P_{e}\right)=\frac{P_{e}-A_{e}}{1-P_{e} A_{e}}
$$

If the electron beam is unpolarized, then

$$
P_{\tau}=\frac{-A_{\tau}-A_{e}\left(2 \cos \theta /\left(1+\cos ^{2} \theta\right)\right)}{1+A_{\tau} A_{e}\left(2 \cos \theta /\left(1+\cos ^{2} \theta\right)\right)}
$$

A measurement of the polar angle distribution of the final-state fermion polarization can be fit to Formula 1.69 , with $A_{+}$and $A_{\epsilon}$ as independent parameters. This fit provides very nearly uncorrelated measurements of $A_{\varepsilon}$ and $A_{\tau}$. The reason for this separation is that the denominator of Equation 1.69 is very near unity, which enables the average polarization to yield information about $A_{\tau}$ while the angular dependence yields information about $A_{e}$

The reason the $Z^{0} \rightarrow \tau^{+} \tau^{-}$channel is chosen is because the average final state polarization of the taus may be measured, owing to the helicity dependence of the distribution of the decay products. In particular, the channels $\tau \rightarrow e \bar{\nu}_{e} \nu_{\tau}, \tau \rightarrow \mu \bar{\nu}_{\mu} \nu_{\tau}$ $\tau \rightarrow \pi \nu_{\tau}, \tau \rightarrow \rho \nu_{\tau}$, and $\tau \rightarrow a_{1} \nu_{\tau}$ contain information about the helicity of the parent $\operatorname{tau}[44]$.

\subsubsection{Polarized Forward-Backward Asymmetries $\tilde{A}_{F B}^{f}$}

The availability of one polarized beam enables an independent combination of cross sections to be formed from Equation 1.61. This combination is often called the "leftright forward-backward asymmetry" because it involves forming a combination that is antisymmetric in both beam helicity and $\cos \theta$, and is denoted $\tilde{A}_{F B}^{f}$ :

$$
\tilde{A}_{F B}^{f}=\frac{\sigma_{F L}^{f}-\sigma_{B L}^{f}+\sigma_{B R}^{f}-\sigma_{F R}^{f}}{\sigma_{F L}^{f}+\sigma_{B L}^{f}+\sigma_{B R}^{f}+\sigma_{F R}^{f}},
$$

where $L$ and $R$ refer to the helicity of the incident electron beam; $F$ and $B$ have the same definitions as in Equations 1.65 and 1.64. The positron beam is assumed to be unpolarized. If the electron beam has polarization $P_{e}$, then this asymmetry can be expressed in terms of the coupling asymmetry using Equation 1.61:

$$
\tilde{A}_{F B}^{f}=\frac{3}{4} P_{e} A_{f}
$$

Measuring this asymmetry was first proposed by Blondel, Lynn, Renard, and Verzeg nassi [39] in 1988, and SLD's measurements are described in References [45]. [46]. [47], [48], [49], [50], and this thesis.

One of the most important reasons for studying this asymmetry is that it is independent of the asymmetry in the couplings of the initial state. While the combination $A_{\varepsilon} A_{f}$ can be measured for various fermions $f$ at LEP, SLD is able to factor that expression and measure $A_{e}$ and $A_{f}$ independently. The importance of this arises from the need to constrain corrections to the $Z f f$ vertex independently of those to the $Z$ ee vertex, because couplings to new particles at at the vertex could be different.

It is also the case that for the particular final state of $b \bar{b}$, the dependence on $\sin ^{2} \theta_{W}$ is very small, as can be calculated at Born level from Equation 1.59. Radiative corrections do not modify the dependence of $A_{b}$ on $\sin ^{2} \theta_{w}$ much, as can be seen in Table 1.4. This is simply a re-expression of the fact that $A_{b}$ is relatively insensitive 
to propagator corrections, in part due to the $b$ quark's small coupling to the photon. As will be shown later, Standard Model vertex corrections also have very little effect on $A_{b}$. For these reasons, the Standard Model has a very narrow range of predictions of $A_{b}$ as a function of $m_{\text {top }}$ and $m_{\text {Higgs. }}$. If any discrepancy were found in its value, it almost certainly is a sign of new phenomena.

Naturally, the argument for the unpolarized case, found in Equation 1.66, which cancels the detector acceptance as a function of polar angle, also applies to this asymmetry:

$\tilde{A}_{F B}^{f}(|\cos \theta|)=\frac{\sigma_{L}^{f}(\cos \theta)-\sigma_{L}^{f}(-\cos \theta)+\sigma_{R}^{f}(-\cos \theta)-\sigma_{R}^{f}(\cos \theta)}{\sigma_{L}^{f}(\cos \theta)+\sigma_{L}^{f}(-\cos \theta)+\sigma_{R}^{f}(-\cos \theta)+\sigma_{R}^{f}(\cos \theta)}=P_{e} A_{f} \frac{2|\cos \theta|}{1+\cos ^{2} \theta}$.

The energy dependence of $A_{L R}^{b}$, shown in Figure 1.4, is minimal, due largely to the fact that the $\gamma-Z$ interference is small. Energies deviating from the $Z^{0}$ pole energy increase the relative fraction of right-handed coupling of the $b$ quark to the electroweak neutral current, owing to the larger contribution of the photon exchange graph.

\subsection{Radiative Corrections}

Radiative corrections modify the Born-level cross-sections and asymmetries and must be included in models in order to fit observations. Some corrections arise from the ordinary physics of QED and QCD, and others are modifications arising from particles that have yet to be discovered. It is important to correct for the known effects, so as to arrive at unbiased estimations of what effect new physics has on the measurements.

\subsubsection{Bremsstrahlung}

Bremsstrahlung, the radiation of energy from accelerated charges, has classical and quantum-mechanical pictures. This radiation can take two forms - initial state radiation (ISR) of photons from the incoming electron and positron, and final-state radiation (FSR), primarily of gluons, from quarks. Quarks also couple to the electromagnetic field, but the radiation cross-section is suppressed by $\left(\alpha_{E M} / \alpha_{s}\right)^{2}$, in

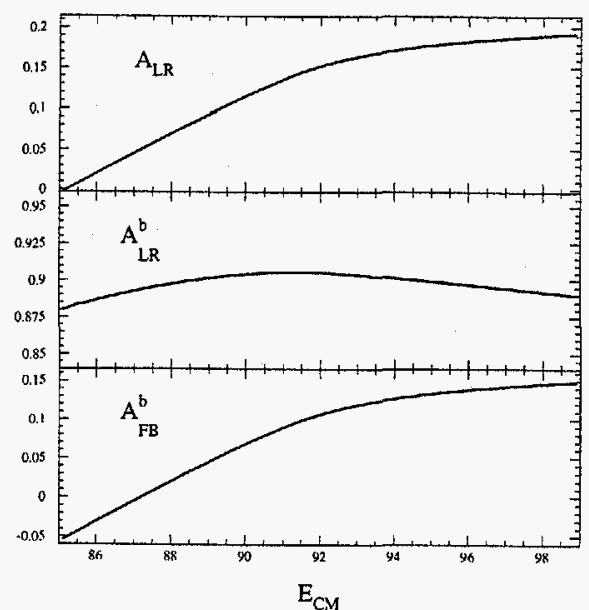

Figure 1.4: Dependence of the asymmetries $A_{L R}, A_{L R}^{b}$, and $A_{F B}^{b}$ on the center-of mass energy. Results are obtained from ZFITTER [51] using $\alpha_{s}=0.118, m_{t}=180, m_{\text {Higgs }}=300$, and $m_{Z}=91.187$.

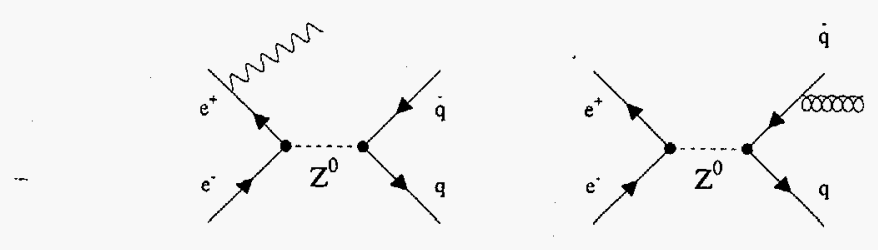

Figure 1.5: Lowest-order bremsstrahlung contributions to $e^{+} e^{-}-Z^{0} \rightarrow q \bar{q}-$ initial state photon radiation, and final-state gluon radiation. 


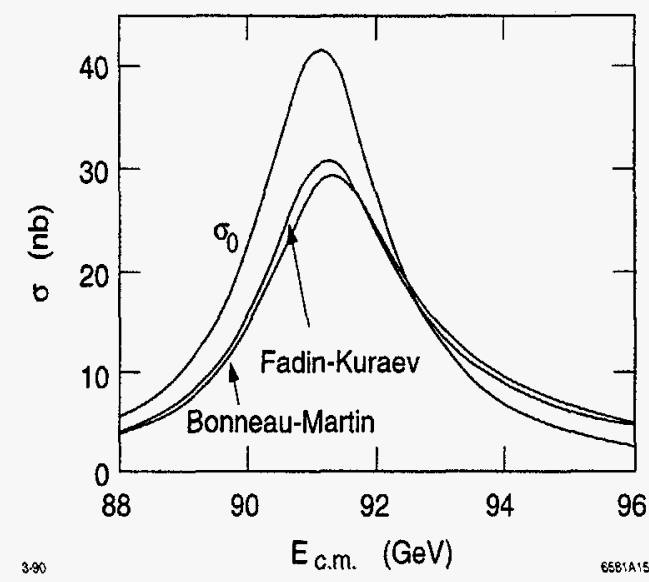

Figure 1.6: Corrections to the $Z^{0}$ lineshape from ISR. The uncorrected cross section is denoted $\sigma_{0}$. From reference [52].

addition to the fractional charge on the quark. Initial-state radiation photons travel predominantly down the beampipe and escape undetected. The effect of initial-state radiation on the collision is largely to reduce the center-of-mass energy and to boost the $Z^{0}$ decay products in the laboratory frame. The width of the $Z^{0}$ resonance is broadened by ISR, and the peak cross-section is shifted up in energy, owing to an average energy loss of the beam. The cross-section above resonance is also enhanced over the cross-section an equal energy below resonance owing to the ease with which the excess energy may be lost due to ISR.

The corrections to the measurement of $A_{b}$ due to ISR, photon exchange, and vertex corrections have been calculated using ZFITTER [51], and by Renard, Blondel, and Verzegnassi [40] using EXPOSTAR [53]. QED radiative effects account for a downward relative shift of $\sim 0.17 \%$ in $A_{b}$ and the measurements in this thesis will be corrected for this.

The other bremsstrahlung contribution, final-state gluon radiation, does not affect the production of the $Z^{0}$, but only its decay. It therefore does not move the energy of the peak cross-section, but it does, however, change the width. This is because hard gluon radiation opens more final quantum states into which the $Z^{0}$ may decay and thus reduces the lifetime. But the correction is only realized for $q \bar{q}$ final states, and it thus affects the partial widths of $Z^{0}$ decay. The magnitude of the effect is given by

$$
\Gamma_{Z^{0} \rightarrow q \bar{q}} \rightarrow \Gamma_{Z^{0} \rightarrow q \bar{q}}\left(1+\frac{\alpha_{s}}{\pi}\right) .
$$

Measurements of $R_{b}$ and $A_{b}$ are fortunately insensitive to this shift in the overall scale of the cross-section induced by hard gluon radiation, due to cancelation in the numerators and denominators of the measurements. Final-state radiation has an additional effect on the observed distributions, though - it smears the quark axis in the detector. Because asymmetry measurements rely on knowing the decay axis of the $Z^{0}$, final-state radiation may modify the measured asymmetry by rescattering quarks from one polar angle to another. These corrections have been calculated to second order for the case of $A_{F B}^{b}$ [54][55], and the same corrections apply to the polarized forward-backward asymmetry [56]. Other calculations of the dependence of this correction on polar angle and on $m_{b}$ are given in References [58] and [57].

\subsubsection{Vertex and Propagator Corrections}

The discussion of bremsstrahlung above would be incomplete without vertex corrections in QED and QCD, owing to the fact that the diagrams of Figure 1.5 have an infrared divergence and lack gauge invariance. The contributions from the diagrams of Figure 1.7 complete the leading-order picture of bremsstrahlung, although they are vertex corrections. Other vertex corrections due to new physics will be dealt with in Section 1.6.

Hard vacuum polarization corrections in the propagator modify the structure of the reaction $e^{+} e^{-} \rightarrow f \bar{f}$. These are shown schematically in Figure 1.8. The first and third lines of Figure 1.8 are simply self-energy renormalizations of the $\gamma$ and $Z^{0}$ propagators, although they affect the relative strengths of their contributions and their interference term. The first set of corrections causes the electromagnetic coupling $\alpha$ to run with energy [59]. The second line of Figure 1.8 alters the helicity structure of the interaction, because the $Z f f$ coupling violates parity while the $\gamma f f$ coupling does not. It is these diagrams that lend the measurements of $A_{e}$ via $A_{L R}$ and 

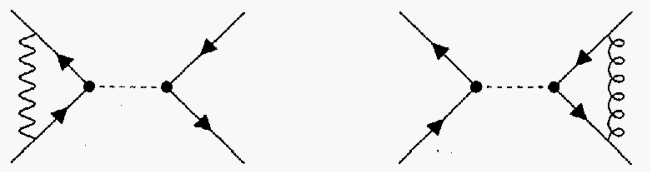

Figure 1.7: Leading order QED and QCD vertex corrections.

$$
\begin{aligned}
& \gamma \operatorname{man} \gamma=i e^{2} \Pi_{Q Q} g^{\mu \nu}+\ldots \\
& Z \operatorname{mnam} \gamma=i \frac{e^{2}}{c s}\left(\Pi_{3 Q}-s^{2} \Pi_{Q Q}\right) g^{\mu \nu}+\ldots \\
& Z \operatorname{mun} Z=i \frac{e^{2}}{c^{2} s^{2}}\left(\Pi_{33}-2 s^{2} \Pi_{3 Q}+s^{4} \Pi_{\infty}\right) g^{\mu \nu}+\ldots
\end{aligned}
$$

Figure 1.8: Propagator corrections to the electroweak neutral current. Here, $s^{2} \equiv \sin ^{2} \theta_{W}$, and $c^{2} \equiv \cos ^{2} \theta_{W}$.

other asymmetries their interest, because the propagator loops may contain $t$ quarks or Higgs particles. The effect of oblique corrections is mostly to modify $\sin ^{2} \theta_{W}$ : although Peskin and Takeuchi have parameterized their possible effects in a model independent manner using three independent variables $S, T$, and $U$ [60],[61]. The $b$ quark couples much less strongly to the photon, and so $A_{b}$ is less sensitive to the propagator corrections than $A_{\varepsilon}$ or $A_{c}$
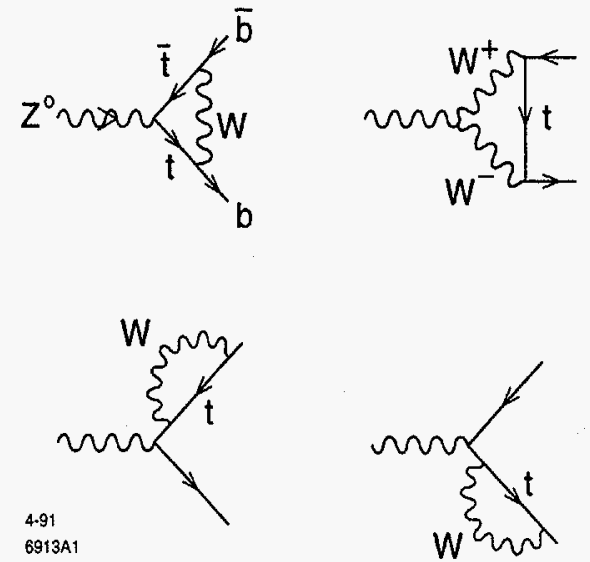

Figure 1.9: Vertex corrections to $Z^{0} \rightarrow b \bar{b}$ within the Standard Model

\subsection{Sensitivity of $A_{b}$ to New Phenomena}

The two observables $R_{b}$ and $A_{b}$ are enough to determine fully the couplings of the $b$ quark to the $Z^{0}$. There are two parameters in the $Z^{0} \rightarrow b \vec{b}$ coupling in the Standard Model; $v_{b}$ and $a_{b}$, or alternatively $\sigma_{L}(b)$ and $\sigma_{R}(b)$, or a strength and an asymmetry. To measure both $R_{b}$ and $A_{b}$ is to define the $Z b b$ vertex and measure corrections to it.

The observable $A_{b}$ is quite remarkable in that it is insensitive to the collision energy, mostly insensitive to $\sin ^{2} \theta_{W}$, and experimentally a very clean number to measure. The Standard Model has a prediction of its value that is very rigid with respect to variations of the model parameters. In particular, $A_{b}$ is insensitive to standard one-loop vertex corrections, shown in Figure 1.9. The reason for this insensitivity is that the $W^{ \pm}$couples exclusively to left-handed fermions, and so the corrections affect only the left-handed coupling of the $b$ to the $Z^{0}$. The sensitivity in $A_{b}$, though, is to the right-handed coupling, as small changes to the left-handed coupling are diluted by the large value of the left-handed coupling. It is for these reasons that $A_{b}$ is an ideal probe of physics beyond the Standard Model, because it is only such new physics that can cause deviations from its expected value. 


\subsubsection{Additional Z Bosons}

Several models involving extended gauge groups propose the existence of additional $Z$ bosons. The effect of such a $Z^{\prime}$ on the value of $A_{b}$ could be profound, if the energy of the interaction were tuned to the $Z^{\prime}$ resonance, and the $Z^{\prime}$ coupled strongly to both the initial state $e^{+} e^{-}$and to the $b \bar{b}$ final state.

For the same reason that the $\gamma-Z^{0}$ interference vanishes on the $Z^{0}$ pole for this asymmetry, (see Equations 1.50 and 1.51), the effect of an interference term with a. $Z^{\prime}$ also vanishes on the $Z^{0}$ pole at tree level. Experiments far from the poles of resonances are more sensitive to $Z^{\prime}$ interactions. However, if the $Z^{\prime}$ mixed with the $Z^{0}$ at the one-loop level, it may have a visible effect on the $Z^{0}$ resonance via a propagator correction. This would have a different effect on $\sin ^{2} \theta_{W}$ for the $b$ quark than it would for the electron, and a measurement of both $A_{c}$ and $A_{b}$ would be valuable in disentangling it.

\subsubsection{Minimal Supersymmetric Model}

The Minimal Supersymmetric Model (MSSM) is an extension to the Minimal Standard Model with "superpartners" for each observed particle. The superpartner of each Fermion is a Boson, and vice versa. In the MSSM, two separate Higgs doublets are required, in order to give masses to the top and bottom quarks. Corrections to both $R_{b}$ and $A_{b}$ have been calculated in the MSSM by Boulware and Finnell [62]. They have noted that $A_{b}$ receives negligible corrections from the supersymmetric sector unless the Higgs doublets have very different vacuum expectation values. If $v_{1}$ and $v_{2}$ are the vacuum expectation values of the two Higgs doublets, and

$$
\tan \beta \equiv v_{2} / v_{1}
$$

then the bottom Yukawa coupling constant $\lambda_{b} \propto m_{b} / \cos \beta \simeq m_{b} \tan \beta$ becomes large in the large $\tan \beta$ limit. Supersymmetric contributions from loops with charginos, shown in Figure 1.10, which have right-handed components proportional to $\lambda_{b}$, become significant.

In addition, loops containing neutralinos, neutral gauginos, and Higgsinos have

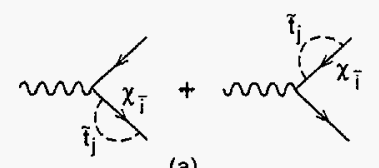

(a)

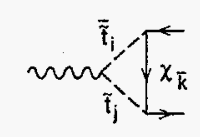

69:3as (b)

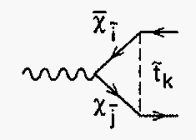

(c) 49,

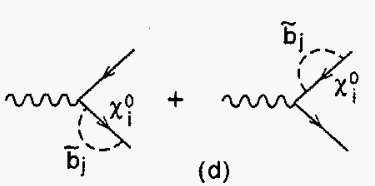

(d)

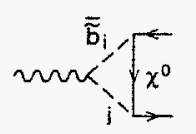

(e)

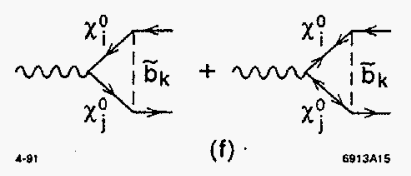

Figure 1.10: Chargino diagrams $(\mathrm{a}-\mathrm{c})$ and neutralino diagrams $(\mathrm{d}-\mathrm{f})$ contributing to $Z^{0}-$ $b \bar{b}$ in the Minimal Supersymmetric Model.

left-handed and right-handed couplings to the $b$ proportional to $\lambda_{b}$; the relevant diagrams are shown in Figure 1.10. Two additional parameters of the MSSM are relerant as the corrections to $A_{b}$ depend strongly on their values. These are the coupling $\mu$ between the two Higgs fields and a supersymmetry breaking Wino mass parameter $M$. The corrections to $A_{b}$ are shown in Figure 1.11 as functions of these two variables. These corrections have been calculated at $\tan \beta=70$, and scale proportionally with $\tan ^{2} \beta$.

\subsubsection{Anomalous Couplings}

Recently, T. Rizzo [63] has parameterized the effects of anomalous electric and magnetic dipole moment couplings between the $b$ quark and the $Z^{0}$. An electric dipole moment of the $b$ quark would violate $C P$ symmetry. While this experiment does not observe a $C P$-violating asymmetry, nonetheless it is able to put limits on the electric dipole moment of the $b$ quark.

Two additional parameters are added to the Standard Model Lagrangian, $k$ and $\tilde{\kappa}$, which are the real parts of the magnetic and electric dipole form factors evaluated 


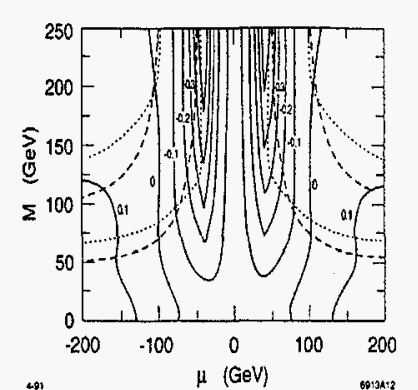

Figure 1.11: Contours showing 100 times the contribution to $A_{b}$ from neutralinos, for $\tan \beta=70$. From Boulware and Finnell [62].

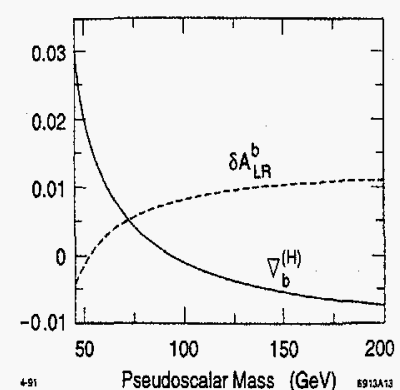

Figure 1.12: Corrections to $R_{6}$ and $A_{b}$ from charged and neutral scalars for $\tan \beta=70$ From Boulware and Finnell [62]. at $q^{2}=M_{z}^{2}$. The Lagrangian expressing the interaction of a fermion $f$ to the $Z^{0}$ gains an additional term containing these

$$
\mathcal{L}_{i \pi t}=\frac{g}{2 \cos \theta_{W}} \bar{f}\left[\gamma_{\mu}\left(v_{f}-a_{f} \gamma_{5}\right)+\frac{i}{2 m_{f}} \sigma_{\mu \nu} q^{\nu}\left(\kappa_{f}-i \tilde{\kappa}_{f} \gamma_{5}\right)\right] f Z^{\mu},
$$

where $g$ is the weak coupling constant, $m_{f}$ is the mass of the final-state fermion, and $q$ is the four-momentum of the $Z^{0}$. The polarized differential cross-section can then be derived in the limit that $m_{f} \ll m_{Z}$.

$$
\begin{gathered}
\frac{d \sigma}{d \cos \theta} \propto\left(1-A_{\epsilon} P_{e}\right)\left[1+\cos ^{2} \theta+2\left(A_{\epsilon}-P_{\epsilon}\right)\left(A_{f}+\frac{2 \kappa_{f} a_{f}}{v_{f}^{2}+a_{f}^{2}}\right) \cos \theta+\right. \\
\left.\frac{1}{v_{f}^{2}+a_{f}^{2}}\left(\frac{m_{Z}^{2}}{4 m_{f}^{2}}\left(\kappa_{f}^{2}+\tilde{\kappa}_{f}^{2}\right) \sin ^{2} \theta+\kappa_{f}^{2}+\tilde{\kappa}_{f}^{2}+4 v_{f} \kappa_{f}\right)\right]
\end{gathered}
$$

which can be compared with Equation 1.61. The modified value of $A_{b}$ measured by the left-right forward-backward asymmetry is then

$$
A_{b}=\frac{2\left(v_{f} a_{f}+\kappa_{f} a_{f}\right)}{v_{f}^{2}+a_{f}^{2}+\frac{3}{4}\left(\frac{m_{z}^{2}}{4 m_{f}^{2}}\left(\kappa_{f}^{2}+\tilde{\kappa}_{f}^{2}\right)+\kappa_{f}^{2}-\tilde{\kappa}_{f}^{2}+4 v_{f} \kappa_{f}\right)} .
$$

\section{Chapter 2}

\section{Current Experimental State}

There is an intense worldwide program to investigate the parity-violating couplings of fermions to the $Z^{0}$. Information about the asymmetry of the $b$ quark coupling has been obtained at the four LEP experiments at CERN. The LEP accelerator has unpolarized electron and positron beams, so the measurable asymmetry $A_{F B}^{b}$ is proportional to the product of the electron coupling asymmetry and the $b$ coupling asymmetry. This chapter presents extractions of $A_{b}$ from the LEP measurements, using an average $A_{e}$ derived from LEP and SLD measurements. Other information on $A_{b}$ comes from SLD itself, taking advantage of the semileptonic decays of the $B$ hadrons.

Lower energy experiments also measure parity-violating couplings, largely by virtue of the interference between the photon and $Z^{0}$-exchange diagrams. While not direct measurements of $A_{b}$, these are provided for comparison.

\subsection{Measurements of $A_{b}$ With Leptons}

Of the measurements using the semileptonic decay modes of $B$ hadrons, SLD's contribution is the most direct. The left-right forward-backward asymmetry is formed in a similar fashion to the one employed in this thesis. This measurement and similar ones at LEP use hadronic events containing high momentum leptons with a large component of that momentum transverse to their respective jet axes. These measurements 
usually extract both $A_{b}$ and $A_{c}$ using a maximum-likelihood technique, where each lepton is assigned a probability of having originated at a $B$ decay vertex, a cascade charm decay, a charm decay vertex in a $Z^{0} \rightarrow c \bar{c}$ event, and also the probability the lepton was a misidentified charged hadron. The SLD measurements are described in detail in References [45], [46] and [47].

The measured values for SLD are

$$
\begin{aligned}
& A_{b}=0.83 \pm 0.12 \text { (stat.) } \pm 0.07 \text { (syst.) muons } \\
& A_{b}=0.87 \pm 0.12 \text { (stat.) } \pm 0.10 \text { (syst.) } \text { electrons } \\
& A_{b}=0.85 \pm 0.09 \text { (stat.) } \pm 0.08 \text { (syst.) } \text { combined. }
\end{aligned}
$$

and

$$
\begin{array}{ll}
A_{c}=0.43 \pm 0.15 \text { (stat.) } \pm 0.12 \text { (syst.) muons } \\
A_{c}=0.46 \pm 0.20 \text { (stat.) } \pm 0.18 \text { (syst.) } & \text { electrons } \\
A_{c}=0.44 \pm 0.12 \text { (stat.) } \pm 0.14 \text { (syst.) } & \text { combined. }
\end{array}
$$

The current measurements from the four LEP experiments of $A_{F B}^{b}$ using semileptonic $B$ decays are given in Table 2.2. The LEP experiments do not correct separately for gluon radiation, photon radiation, $\gamma-Z^{0}$ interference, or the $\gamma$-exchange contributions. Instead, they report raw asymmetry values at the LEP ring energy, which are averaged and then corrected for those effects to arrive at an average $A_{F B}^{0}$. In order to extract a per-experiment measurement of $A_{b}$, then, one must apply these corrections and also divide by $\frac{3}{4} A_{\varepsilon}$.

The $A_{e}$ to be used must be chosen judiciously. As described in Chapter 1, the lepton forward-backward asymmetries, the tau polarization asymmetry; $A_{L R}$, and the quark forward-backward asymmetries all are sensitive to $\sin ^{2} \theta_{W}$. It is common to form an average $\sin ^{2} \theta_{W}$ with the assumption that the Minimal Standard Model holds, and then to extract a prediction of $A_{e}$. To use this $A_{e}$ to extract $A_{b}$ from $A_{F B}^{b}$ would be circular, in that $A_{F B}^{b}$ is one of the most important contributions to the determination of $\sin ^{2} \theta_{W}$ and therefore of $A_{e}$, and its inclusion in the fit assumes $A_{b}$ takes its standard value.

The prescription is to average the $A_{e}$ values only from purely leptonic measurements - SLD's $A_{L R}$, the forward-backward lepton asymmetries from LEP (assuming
Table 2.1: Current leptonic measurements of $A_{e}$ used to extract $A_{b}$ from $A_{F B}^{b}$. From Reference [64].

\begin{tabular}{|lc|}
\hline Measurement & $A_{e}$ \\
\hline SLD $A_{L R}$ & $0.1551 \pm 0.0040$ \\
LEP $A_{F B}^{l}$ & $0.1514 \pm 0.0053$ \\
LEP $P_{\tau}$ & $0.1406 \pm 0.0057$ \\
\hline Average & $0.1506 \pm 0.0028$ \\
\hline
\end{tabular}

Table 2.2: Measurements from the four LEP experiments of $A_{F B}^{b}$ from semileptonic $B$ decays, given in Reference [65], uncorrected for QCD, QED, and $\gamma-Z$ interference. The values of $A_{b}$ are extracted using the value of $A_{e}$ from Table 2.1, and are also corrected for radiative effects and photon exchange.

\begin{tabular}{|lccc|}
\hline Experiment & Data Sample & $A_{F B}^{b}$ & $A_{b}$ \\
\hline ALEPH & $1990-1993$ & $0.0846 \pm 0.0068 \pm 0.0022$ & $0.800 \pm 0.065$ \\
DELPHI & $1991-1994$ & $0.1049 \pm 0.0076 \pm 0.0035$ & $0.980 \pm 0.077$ \\
L3 & $1990-1993$ & $0.103 \pm 0.010 \pm 0.004$ & $0.963 \pm 0.097$ \\
OPAL & $1990-1994$ & $0.1030 \pm 0.0090 \pm 0.0040$ & $0.963 \pm 0.089$ \\
\hline
\end{tabular}

lepton universality), and $A_{c}$ from the tau polarization asymmetry. These are shown in Table 2.1, using data from Reference [64].

\subsection{Measurements of $A_{b}$ With Momentum-Weigh- - ted Charge}

The second technique of measuring $A_{b}$ at the $Z^{0}$ also involves forming the forwardbackward asymmetry and dividing by $\frac{3}{4} A_{\varepsilon}$, but the asymmetry is found using momentum-weighted track charge to distinguish the $b$ direction from the $\bar{b}$ direction. The 
Table 2.3: Measurements from three of the four LEP experiments of $A_{F B}^{b}$ using momentumweighted charge in lifetime-tagged $Z^{0} \rightarrow b \bar{b}$ samples, as reported at the 1995 Europhysics Conference [65]. The values of $A_{b}$ extracted assume $A_{\varepsilon}=0.1506 \pm 0.0028$.

\begin{tabular}{|lccc|}
\hline Experiment & Data Sample & $A_{F B}^{b}$ & $A_{b}$ \\
\hline ALEPH & $1991-93$ & $0.0992 \pm 0.0084 \pm 0.0046$ & $0.930 \pm 0.087$ \\
DELPHI & $1991-1994$ & $0.0999 \pm 0.0072 \pm 0.0038$ & $0.936 \pm 0.074$ \\
OPAL & $1991-1994$ & $0.0963 \pm 0.0067 \pm 0.0038$ & $0.904 \pm 0.071$ \\
\hline
\end{tabular}

sample of $Z^{0} \rightarrow b \vec{b}$ decays is identified with the aid of precision silicon microvertex detectors. The details of the SLD approach to this technique are presented in Chapters 6 and 7 .

Because of systematic errors inherent in using Monte Carlo simulations to determine the effectiveness of the momentum-weighted track charge technique, the three LEP experiments that contribute these measurements use distributions from data to calibrate the technique. Two of the LEP experiments, ALEPH and OPAL, follow a prescription similar to, but less sophisticated than, the one presented in Chapter $\bar{i}$. The differences are that the SLD technique takes into account the $\cos \theta$ dependence of the asymmetry and the correct-sign probability, as well as parameterizing the correctsign probability as a function of the momentum-weighted charge. The SLD technique also accounts for the QCD correction in a $\cos \theta$-dependent manner, while the OPAL technique [66] applies an overall correction, and the ALEPH technique [67] ignores it altogether. The measurement from DELPHI [68] takes a different approach by tagging a high- $\left(p, p_{\perp}\right)$ lepton in one hemisphere and examining the momentum-weighted charge in the opposite hemisphere. This distribution is then used in the inclusive lifetime-tagged sample to measure $A_{F B}^{b}$.

\subsection{Combined LEP $A_{b}$}

The combined value of $A_{F B}^{0, b}$, corrected for radiation and $\gamma$-exchange and reported at the 1995 Europhysics Conference [65] is $0.0997 \pm 0.0031$, resulting in an inferred $A_{b}$
Table 2.4: Measurements of $A_{F B}^{b}$ at $\sqrt{s}=57.8 \mathrm{GeV}$ from the three TRISTAN experiments Correction for $B$ meson mixing is not included in these measurements, but would enlarge the values by a factor of $\sim 1.3$ if it were.

\begin{tabular}{|lcc|}
\hline Experiment & $A_{F B}^{b}$ & Reference \\
\hline AMY & $-0.59 \pm 0.09 \pm 0.09$ & {$[69]$} \\
VENUS & $-0.55 \pm 0.15 \pm 0.08$ & {$[70]$} \\
TOPAZ & $-0.55 \pm 0.27 \pm 0.07$ & {$[71]$} \\
\hline
\end{tabular}

of $0.883 \pm 0.032$. This value can be compared with the Standard Model prediction of 0.935 . The measurement presented in this thesis compares favorably with individual measurements from the four LEP experiments, although the total error on the combined value is much less than that on any one contribution.

\section{$2.4 \quad A_{F B}^{b}$ at Other Energies}

A compilation of measurements of $A_{F B}^{b}$ at energies up to the $Z^{0}$ pole energy can be found in Reference [69]. The energy dependence of $A_{F B}$ follows the general behavior that is expected from the Standard Model predictions, outlined in Chapter 1. The value of $A_{F B}$, as measured off the $Z^{0}$ pole, is not strictly proportional to $A_{b}$, due to the presence of parity violation in both the direct $Z^{0}$ exchange term and the interference term, and the lack of it in the photon exchange term.

The measurements of $A_{F B}^{b}$, as measured at $\sqrt{s}=57.8 \mathrm{GeV}$ at TRISTAN, are given in Table 2.4. They are also are displayed with the Standard Model predictions. and data from PEP, PETRA, and LEP, in Figure 2.1. These measurements were made with the high- $\left(p, p_{\perp}\right)$ lepton technique. 


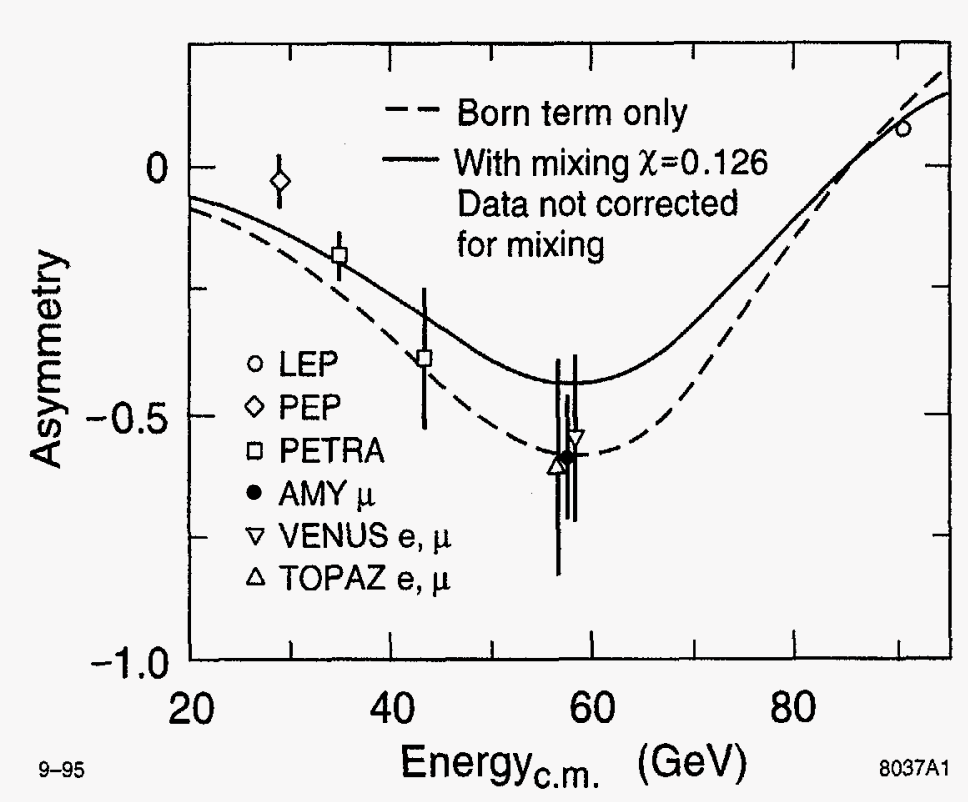

Figure 2.1: Measurements of $A_{F B}^{b}$ as a function of $E_{C M}$, measured at PEP, PETRA, TRISTAN, and LEP, as compiled in Reference [69]. The data are uncorrected for $B$ meson mixing, and can be compared with the model incorporating mixing (solid line).

\section{Chapter 3}

\section{Experimental Apparatus: SLC and the Compton Polarimeter}

To perform a measurement of $A_{b}$ on the $Z^{0}$ resonance, the initial quantum state of the $e^{+}$and $e^{-}$must be carefully prepared and characterized. The SLAC Linear Collider (SLC), comprised of a polarized electron gun, a linear accelerator, a positron source, damping rings, collider arcs, and final focus optics has been commissioned and has been operating since 1989 producing $Z^{0}$ bosons. To measure the properties of the beam, the SLC itself has diagnostic tools at every stage, but the most important physical quantities must be measured as close to the detector as possible. The relevant beam parameters are energy, energy spread, luminosity, and polarization.

\subsection{The SLAC Linear Collider}

The need to accelerate an electron beam and a positron beam to an energy sufficient to collide them at the $Z^{0}$ resonance, and the need to focus them tightly enough to produce enough luminosity to carry out sensitive studies of the electroweak interaction, together govern the design of the SLC [72]. Several innovations in accelerator technology were crucial in making SLC a successful machine for particle physics studies. SLC is the first $e^{+} e^{-}$linear collider, and at the moment shares with LEP the distinction of being the highest energy $e^{+} e^{-}$accelerator yet built. 


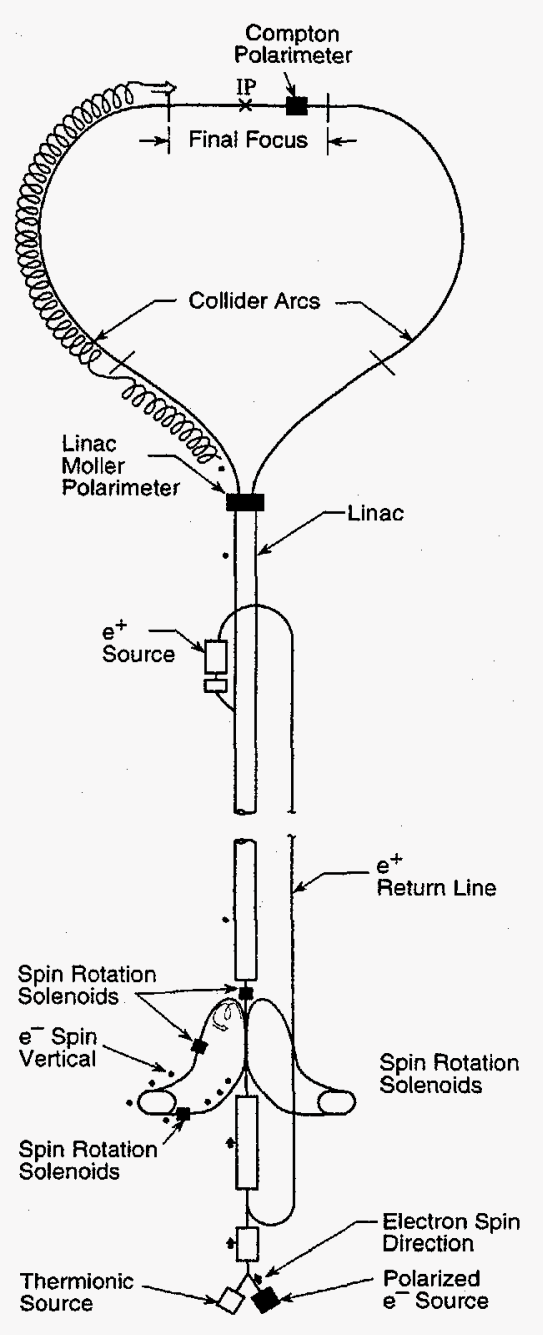

Figure 3.1: Layout of the spin-polarized SLC, showing the beam transport lines and polarization orientation.

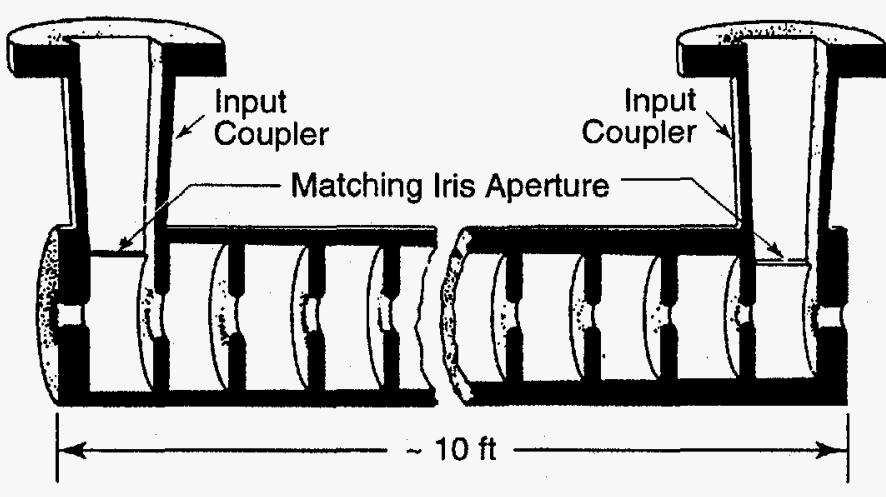

$10-95$

185A2

Figure 3.2: A 10-foot longitudinal section of the disk-loaded waveguide accelerator structure. The longitudinal period of the structure is 1.378 inches, with a disk thickness of 0.230 inches. so the phase shift in the RF per cavity is $2 \pi / 3$. The cavity diameter varies from 3.286 inches in the first cavity to 3.220 inches the last. The diameter of the aperture in the disks varies from 1.032 inches in the first cavity to 0.752 inch in the last. The taper in the dimensions is required to maintain a constant gradient across the section, after accounting for attenuation and beam loading. From Reference [74].

The electron beam starts at an electron gun containing a strained-lattice galliumarsenide cathode, which photoemits longitudinally polarized electrons when illuminated by circularly polarized laser light [73]. The details of polarized beam production and transport will be given in Section 3.4.

The accelerator consists of two miles of copper disk-loaded waveguide, shown in Figure 3.2. The resonant structure of the accelerator pipe allows a standing wave to exist with a component of the electric field along the direction of the beam. If electrons are introduced at the appropriate phase of the oscillating field, they will receive an acceleration along the beam tube. Positrons injected on the opposite phase can be accelerated in the same direction to the same energy.

The microwave energy for the accelerator is supplied by pulsed $38 \mathrm{MW}, 2.856 \mathrm{GHz}$ klystrons. Each second, the klystrons produce 120 pulses of RF $5.0 \mu \mathrm{s}$ long. The RF 
pulses are nearly doubled in power by the SLED-II cavities [75]*, which shorten the effective pulse duration to $800 \mathrm{~ns}$ while increasing the amplitude. With the SLED-II cavities operating, the electron beam receives $\sim 14.5 \mathrm{MV}$ of energy for every meter of accelerator structure traversed.

Both electrons and positrons must accelerate down the tube on the same pulse. To accomplish this, the electron gun produces two bunches of electrons in quick succession. Both bunches are accelerated to $1.19 \mathrm{GeV}$ in the first section of the accelerator and are shunted into the North Damping Ring (NDR), where they remain until the next RF pulse from the klystrons. The South Damping Ring (SDR) is built to damp positrons made on the previous machine cycle.

The damping rings enable SLC to focus its beams tightly. The electron beam emerges from the gun with a large width and a large angular divergence. Liouville's theorem demands that the total volume of a bunch in position/momentum phase space be conserved by a non-dissipative accelerator. The damping rings introduce a dissipative element - synchrotron radiation - to reduce the phase space volume of the bunches. Short accelerating sections in the rings compensate for radiative losses, and the electrons settle into a stable orbits determined by the damping ring parameters, having lost all knowledge of their prior trajectories before entering the ring. The bunches shorten and reduce their angular divergence in the rings.

It is in the damping rings that the beams are made flat. In the absence of coupling between oscillations in the two transverse directions, the stable orbits of a horizontal ring occupy a region of space that is larger in the horizontal direction than the vertical. SLC typically runs an aspect ratio at the interaction point of 4.6:1 [78].

The damping rings were upgraded between the 1993 and 1994-1995 runs [79]. The main upgrade was to redesign the vacuum chamber to reduce the impedance. This improvement delays the onset of bunch-lengthening instabilities as the bunch population is increased, allowing higher luminosity.

On the next pulse of RF from the klystrons, both bunches of electrons from the NDR and a bunch of positrons from the SDR are extracted and accelerated in the linac. The positron bunch leads the two electron bunches in the linac. The
Table 3.1: Parameters of the SLC damping rings [76][77].

\begin{tabular}{|lc|}
\hline Energy & $1.19 \mathrm{GeV}$ \\
Circumference & $35.270 \mathrm{~m}$ \\
Revolution Frequency & $8500.411 \mathrm{kHz}$ \\
RF Frequency & $714.000 \mathrm{MHz}$ \\
Bending Radius & $2.0372 \mathrm{~m}$ \\
Energy Loss/turn & $93 \mathrm{KeV}$ \\
Damping Time $\tau_{x}$ & $3.32 \pm 0.28 \mathrm{~ms}\left(e^{-}\right)$ \\
& $3.60 \pm 0.15 \mathrm{~ms}\left(e^{+}\right)$ \\
Damping Time $\tau_{y}$ & $4.11 \pm 0.31 \mathrm{~ms}\left(e^{-}\right)$ \\
& $4.17 \pm 0.14 \mathrm{~ms}\left(e^{+}\right)$ \\
\hline
\end{tabular}

trailing bunch of electrons, called the "scavenger bunch," is sent to the positron source, roughly 1.33 miles down the accelerator. After receiving $31 \mathrm{GeV}$ of energy: this bunch strikes a tungsten target, producing an electromagnetic shower. A pulsed solenoid focuses the produced positrons so they may be accepted by a short booster which accelerates them to $200 \mathrm{MeV}$. The positrons are then guided back through the positron return line to the injector, accelerated to $1.19 \mathrm{GeV}$, and steered into the $\mathrm{SDR}$, to provide the positrons for the next machine cycle.

The other two bunches extracted from the damping rings, one each of electrons and positrons, are the luminosity-producing bunches. The short bunch length produced by the damping rings is crucial in reducing the energy spread - a long bunch will sample a larger portion of the accelerating wave, increasing the magnitude of longitudinal oscillations about equilibrium. A technique termed "overcompression," introduced for the 1994-1995 run [80], shortens the bunches further in the transfer line between the damping rings and the accelerator, reducing the energy spread.

A lattice of quadrupole magnets interleaved with the accelerating sections keeps the beam focused within the accelerating field given its finite angular divergence.

" "SLED" stands for SLAC Energy Doubler. 
Within each quadrupole magnet, a beam position monitor (BPM) provides information for feedback control [81] of accelerator parameters such magnet strengths and klystron phase, as well as providing information for online diagnostics. Each BPM also produces a measurement of the beam current.

The beams gain energy from the $1.19 \mathrm{GeV}$ of the damping rings to $46.6 \mathrm{GeV}$ by the time they reach the Beam Switchyard (BSY).

The BSY contains dipole magnets which direct the electrons into the North SLC arc (NARC), and the positrons into the South arc (SARC). The arcs contain a lattice of dipole and quadrupole magnets to keep the beams focused while steering them into collision. The arc tunnels were dug following the local terrain and passing underneath the PEP tunnel. The configuration of magnets needed to guide the beams through the arcs has important consequences on the transport of the electron spin, to be discussed in Section 3.4.5

Collimators at the end of the LINAC and at different places in the arcs remove portions of the bunch that have the wrong energy or have strayed too far from the bunch core. Synchrotron radiation losses in the arcs amount to $1 \mathrm{GeV}$ per electron.

The last section of beampipe before the interaction point (IP) is nearly straight, in order to reduce the beam-related backgrounds in the detector. These straight sections contain magnets designed to bring the beams into focused spots at the IP. Superconducting quadrupoles form the final triplets in order to provide a higher field strength than conventional iron-yoke magnets, and allow operation within the SLD solenoidal field [82].

The final focus optics were upgraded for the 1994-1995 run in order to reduce the chromatic effects on the focal length of the system [83].

A Compton polarimeter, to be described in Section 3.5, continuously measures the electron beam polarization in the South Final Focus (SFF). Energy spectrometers, to be described in Section 3.5.3, measure the energy of the two beams.

Constant feedback keeps the beams in collision at the IP in the face of thermal motion and upstream adjustments. Additionally, every five minutes, horizontal and vertical scans of one beam across the other are made to determine the transverse beam dimensions. The deflection of one beam from the other is measured by BPM's downstream of the IP on each pulse of the scan, and fits are performed to deconvolute the dimensions of the two beams. The slope of the deflection as a function of corrector strength is used to tune the feedback system.

Another detector, the radiative Bhabha counter, detects electrons that have lost a portion of their energy by scattering off of the particles in the other bunch. These lower-energy electrons are overbent in the first bending magnet of the final focus, and are steered into this detector. It provides a fast estimate of beam luminosity for accelerator tuning purposes, although backgrounds preclude its use for precision luminosity measurements.

A Møller polarimeter was installed briefly in the electron extraction line at the end of the 1993 run to cross-check the polarization measured by the Compton polarimeter. Its installation precludes the use of the energy spectrometer and the positron beam, so it can only be used as a check. It has larger systematic errors than the Compton polarimeter and will not be used in this analysis. Another Møller polarimeter, situated in the beam switchyard, can be used more easily - no hardware needs to be moved to switch between physics running and Møller measurements - although it lies on the other side of the NARC, which can precess the electron spin in an arbitrary direction, and also partially depolarize the beam.

\subsection{Performance}

SLC has constantly improved its luminosity since it started producing $Z^{0}$ bosons in 1989 , by a combination of shrinking the beam waists at the collision point, increasing the electron and positron bunch populations, and increasing the reliability of operation. This reliability factor includes not only the fraction of the time the accelerator is on and delivering bunches to the IP, but also the the fraction of the time it is optimally tuned. Feedback mechanisms have greatly reduced the need to dedicate time to tuning the beams [81]. In addition, reduced deadtime from SLD increased the effective luminosity. This was achieved by pipelining the data acquisition, suppressing readout on beam crossings for which the detector was saturated with noise, buffering the logging stream, and streamlining the run switchover procedure. During 


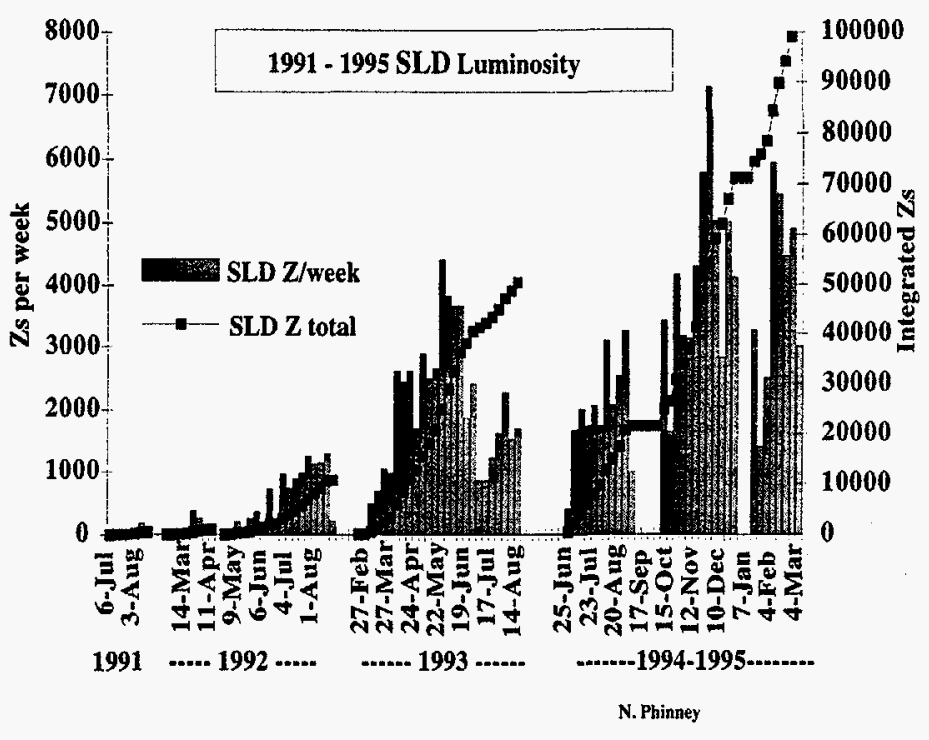

Figure 3.3: Integrated and differential luminosity histories of the SLC.

the 1994-1995 run, typical luminosities achieved were 50-60 $Z^{\circ} /$ hour, while in 1992, $30 Z^{0}$ /hour was more common. A history of the SLC luminosity for the time SLD .was running is given in Figure 3.3.

The polarization plays a crucial role in the ability of SLD to measure asymmetries as the error on $A_{b}$ and $A_{L R}$ scales inversely with $P_{e} \sqrt{\mathcal{L}}$. A bulk GaAs photocathode provided the electron source in 1992, with a realized polarization of $22 \%$. For 1993. a strained GaAs crystal was installed, and in 1994, a thinner strained GaAs crystal took its place. The history of the polarization as a function of the count of collected $Z^{\circ}$ bosons is shown in Figure 3.4

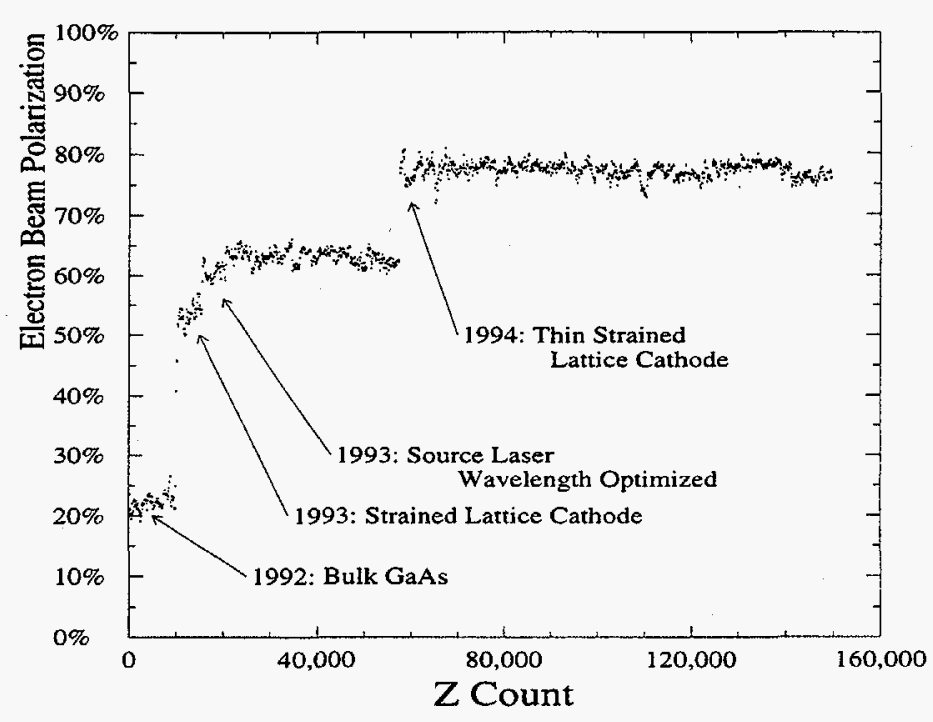

Figure 3.4: History of the electron beam polarization as a function of the count of collected $Z^{0}$ s. Large discontinuities correspond to cathode changes for the different running years.

\subsection{Linear Colliders and Synchrotrons}

The competing experiments at the $Z^{0}$ are at CERN on the LEP ring, a synchrotron $26.66 \mathrm{~km}$ in circumference with four interaction halls. This device has several advantages over the linear collider at SLAC, and several shortcomings. The colliding-beam synchrotron has had a long history as a successful accelerator design since its introduction with the ADONE ring in Italy and the construction of the SPEAR ring at SLAC. The SPEAR ring is small (234 meters in circumference) and operates each beam at energies up to $3.5 \mathrm{GeV}$. The physical phenomenon that dominates the design of these accelerators is energy loss through synchrotron radiation, which dissipates the beam's energy at a rate of

$$
\Delta E \simeq \frac{6 \times 10^{-18}}{r}\left(\frac{E_{\text {beam }}}{m c^{2}}\right)^{4} \mathrm{GeV} / \text { Turn },
$$


where $r$ is the bending radius of curvature of the ring, measured in meters, and $m$ is the mass of the accelerated particles. This energy must be replenished constantly by RF cavities. Assuming constant unit prices for land, tunnels, accelerator components, and electrical power, the optimal size of a synchrotron must increase in proportion to the beam energy squared. The circumference of LEP verifies this relation.

The accelerating portion of a linear collider, on the other hand, dissipates energy to synchrotron radiation only in proportion to the energy to the first power. Furthermore, the accelerator energy is proportional to its length, so all costs scale with energy to the first power. The required bend strength for the SLC arcs is modest ${ }^{\dagger}$. At the $Z^{0}$ resonance and above, linear colliders are smaller and less expensive than synchrotrons of the same energy.

The rate at which new pulses are injected into the SLC is limited to $120 \mathrm{~Hz}$ for two reasons. The first is that the power consumption of the linac scales with the repetition rate as the accelerator structure is re-filled with $R F$ on every pulse. The second is that the pulses require an $\sim \delta \mathrm{ms}$ damping time in the damping rings. and placing more bunches in the damping rings has been shown to introduce bunchlengthening instabilities [85]. This contrasts with the $90 \mathrm{KHz}$ bunch-crossing rate at. LEP, assuming eight bunches for each beam are stored in the ring simultaneously.

What SLC loses in bunch-crossing frequency it must make up for in beam spot size to produce enough collisions for physics analysis. Current performance of the LEP accelerator's spot sizes is $10 \mu \mathrm{m}$ vertically by $150 \mu \mathrm{m}$ horizontally. SLC has a spot size of $0.5 \mu \mathrm{m}$ by $2.3 \mu \mathrm{m}$.

The small spot size of SLC also allows for higher precision measurements of decay lengths near the IP. When the beams are large, the point of decay of the $Z^{0}$ must be measured on each event, subject to detector resolution. Owing to the fact that all of the charged decay products in many events travel in paths nearly parallel to the thrust axis, information about the location of primary vertex along that axis may be very poor. With spots that are small in two dimensions, the primary vertex measurements of many events may be found and averaged [86], providing excellent knowledge of

\footnotetext{
${ }^{\dagger}$ The size of the SLC arcs was determined by the need to keep the emittance growth due to
} synchrotron radiation down, while fitting on SLAC property. the location of the $Z^{0}$ decay on each event. Care must be taken to average events only over times when the beam did not move significantly. The beam motion is usually very small at SLC - large jumps are infrequent and have identifiable causes. Knowledge of the beam position for each event allows determination of the impact parameters of tracks relative to the beams with high accuracy, a necessary ingredient for the analysis presented in this thesis.

The most significant advantage SLC has over LEP for electroweak asymmetry measurements is its ability to polarize its electron beam, to be described in the next section. The positron beam can not be polarized readily because it is produced by an electromagnetic shower in a tungsten target. Furthermore, the positron transport system is not designed to preserve any polarization of the positron beam. The Sokolov-Ternov polarization buildup time [87] in the positron damping ring is $\sim 960 \mathrm{~s}$, while the positrons only spend $16.7 \mathrm{~ms}$ in the ring.

Backgrounds from stray particles tend to be different at linear colliders and synchrotrons. Each sensitive component near the interaction region is susceptible to particular types of backgrounds and not to others. For example, the tracking chambers are sensitive to the presence of synchrotron radiation produced in the final focus optics because high-energy photons scatter on the atomic electrons in the beampipe and detector material. Some electrons are freed with enough energy to travel through the tracking volume and leave an ionization trail. Because their energy is typically very low, the tracks left by these particles are small helices that stay close to the point at which the electron was liberated, predominantly in the inner layers of the chamber. If enough of these electrons are liberated each beam crossing, the chamber will not be able to hold its design voltage. The linear collider design increases the presence of synchrotron radiation near the experiment by demanding that the focusing of the last set of magnets be very strong, in order to reduce the beam widths. This background is present also in synchrotrons, and its magnitude is proportional to the beam current, but the focusing angles are smaller.

Another background found at both types of machines is the presence of muons traveling roughly parallel to the beam. These are produced by the Bethe-Heitler 
mechanism [88] when an electron passes close to a nucleus in some material far upstream from the detector. These muons penetrate the material of the beamline optics, tunnel walls, shielding blocks, and pass through the detector's calorimeter. This background is controlled by minimizing the population of the wide tails of the bunches as they pass by apertures in the beam transport system. Early collimation in the accelerator, as well as control of the energy spread and bunch-to-bunch position and energy jitter help to reduce this background, and it is much less prevalent since SLC first began operation. At a synchrotron, one needs to maintain a large aperture in regions of high dispersion, but the beam tails are clipped soon after injection.

In response to the different operating environment of a linear collider, techniques were developed to optimize the luminosity and reduce accelerator backgrounds. Feedback mechanisms were installed along the accelerator, in the damping rings, in the arcs, and in the final focus region. The SLD detector forms a summary of the signal in each of its major sensitive systems on each pulse of the accelerator and sends this information to the accelerator control center in a useful display on a storage oscilloscope. This allows the accelerator operators to see immediately the impact of every action they take to reduce the backgrounds in the detector, and background reduction has become a routine task that can be performed rapidly and effectively. During the 1993 run, approximately 0.3 muons per beam crossing passed through the calorimeter, and approximately $6 \%$ of the sensitive wires in the drift chamber registered a background pulse. The effect of these backgrounds on physics analysis is very small and will be discussed in Chapters 5 and 6 .

\subsection{Spin Production and Transport}

\subsubsection{Electron gun}

The electron gun, shown in Figure 3.5, consists of a cathode of gallium arsenide on a substrate of gallium arsenide phosphide, to provide lattice strain [73]. It is held at a potential of $-120 \mathrm{kV}$, while the surrounding metal structure in the electron gun is held at ground. The structure of the anode is such that the electrons are given

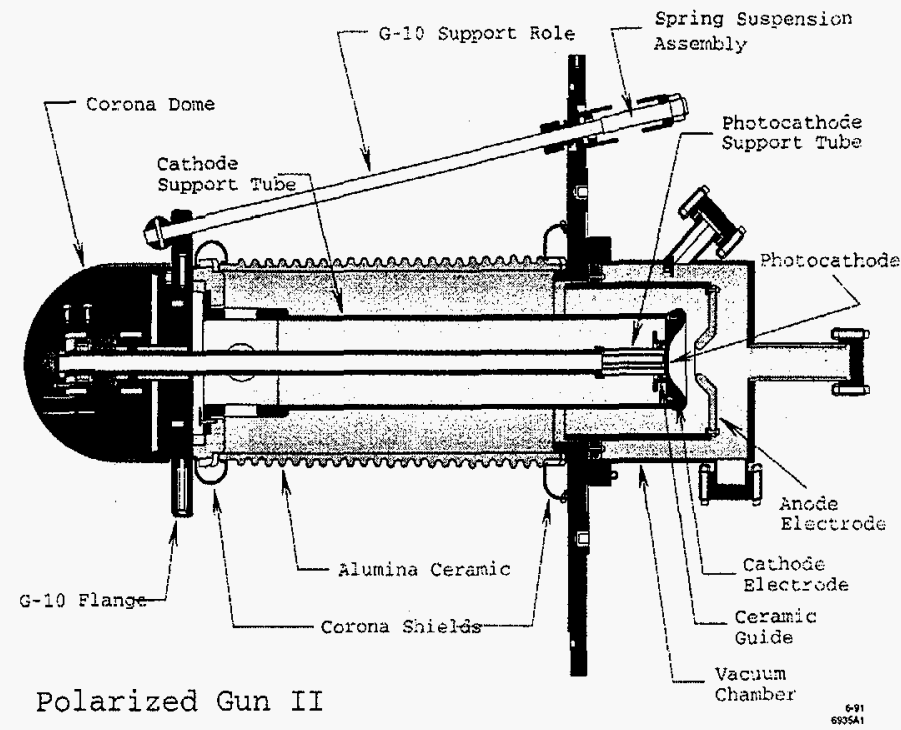

Figure 3.5: The polarized gun used on SLC in 1993. The photocathode used was a strainedlattice GaAs cathode.

nearly the full $120 \mathrm{kV}$ of acceleration so they may be accepted by the first section of the accelerator. With time, the quantum efficiency of the cathode degrades. Roughly every four days during running, a thin layer of cesium, moderated with fluorine, is added to the surface of the cathode to restore optimal quantum efficiency. Every few months, the lifetime of the quantum efficiency after each addition of cesium gets shorter, and the cathode must be baked at high temperature to clean the surface of impurities.

\subsubsection{Photocathode}

The need to reach high electron beam polarizations drove the choice to develop thin, strained GaAs cathodes for SLC [73]. A GaAs cathode will emit polarized electrons if it is illuminated with circularly polarized light, but the magnitude of the polarization 

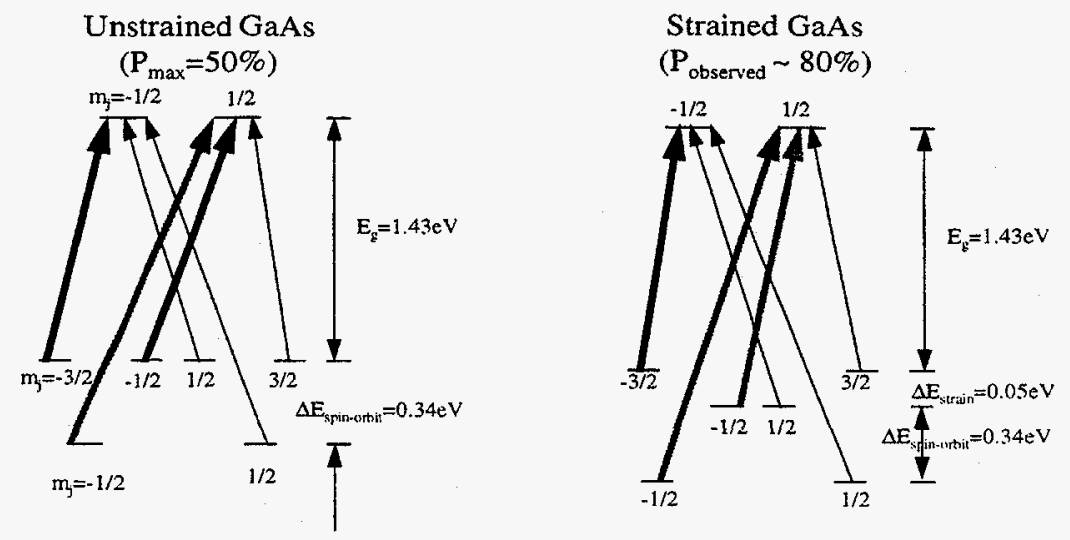

Figure 3.6: Energy levels in unstrained and strained GaAs. The dominant $\Delta L=1$ transitions are shown in bold. The strain breaks the degeneracy between the $m_{j}=-3 / 2$ and $m_{j}=-1 / 2$ levels in this figure, suppressing transitions from the latter levels.

obtainable is determined by the energy states within the crystal, shown in Figure 3.6. The electrons which emerge from the crystal first have to be elevated from the valence band to the conduction band before they may escape the work function of the material. A bulk crystal of GaAs has an important degeneracy between the spin states in the valence band, which is brought about by the crystal symmetry. If circularly polarized light impinges upon the crystal, two transitions of equal splittings are excited. These transitions have a strength ratio of 3:1, owing to a difference in their Clebsch-Gordan coefficients. With no other bias between these two transitions, the maximum available polarization obtainable is $50 \%$.

The energy degeneracy of the different spin states in the valence band can be lifted by breaking the crystal symmetry with a mechanical strain. Placing the GaAs in compression favors the already strong transition over the weaker one. The strain is induced by growing a thin layer of $\mathrm{GaAs}$ on a crystal of GaAsP, which has a slightly. smaller lattice constant. Because the splitting due to the strain is only $0.05 \mathrm{eV}$ while the transition energy is $1.52 \mathrm{eV}$, the additional gain in polarization is dependent on

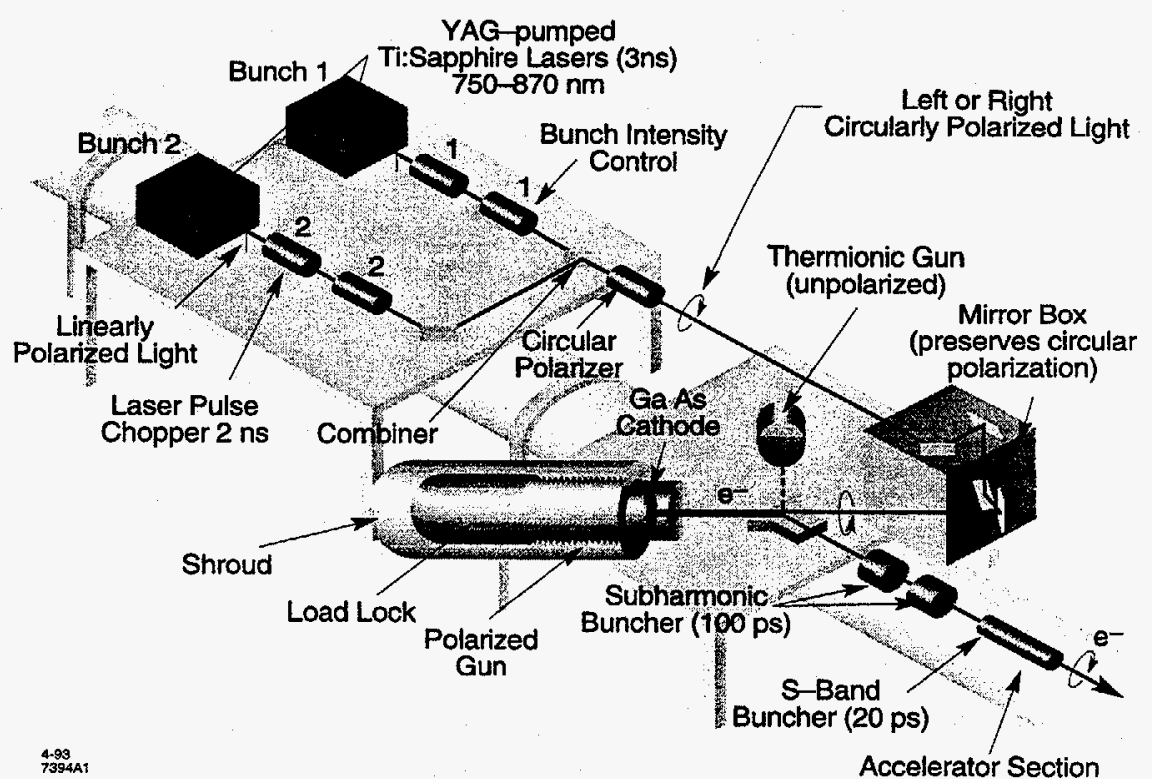

Figure 3.7: Optical layout of the polarized source.

the energy of the photons used. To optimize the polarization, photons of the lowest possible energy are used, consistent with the requirement that the quantum efficiency be high enough to extract sufficient current from the gun. The quantum efficiency can be improved by adding cesium, but also at the cost of lowering the polarization slightly.

The thickness of the strained GaAs layer strongly affects the achievable polarization because the GaAs lattice relaxes with increasing distance from the GaAsP interface [73]. The cathode used in the 1993 run had a thickness of $0.3 \mu \mathrm{m}$ and delivered a beam polarization of $\approx 63 \%$, while the cathode used for 1994-1995 had a thickness of $0.10 \mu \mathrm{m}$ and delivered a polarization of $\approx 77 \%$. 


\subsubsection{Electron source optics}

This cathode emits photoelectrons when light of a wavelength shorter than $\sim 900 \mathrm{~nm}$ impinges on it. Reversing the helicity of the circularly polarized light reverses the helicity of the electron beam. The pulses of light need to be short, intense, and of a controllable wavelength, so YAG-pumped Ti:Sapphire lasers are used. The light is circularly polarized by first linearly polarizing it, and then passing the beam through a Pockels cell. The sign of the voltage on the Pockels cell determines the orientation of its fast axis and slow axis. If these axes are arranged to be at $45^{\circ}$ to the plane of polarization of the laser light, and the phase advance difference between the two axes is $90^{\circ}$, then the resulting light emerges circularly polarized. The sign of the voltage on the Pockels cell, and thus the helicity of the light from the apparatus, is chosen pseudo-randomly ${ }^{\ddagger}$ on each beam pulse in order to minimize systematic effects from periodic behavior of the accelerator.

Two $60 \mathrm{~Hz}$ YAG lasers pump both Ti:Sapphire cavities; their pulses are interlaced so the Ti:Sapphire cavities pulse at $120 \mathrm{~Hz}$. One cavity produces the pulse for the polarized electrons, and is tuned to a frequency of $845 \mathrm{~nm}$ for the 1994-1995 $\mathrm{run}^{\S}$. The other Ti:Sapphire cavity produces $765 \mathrm{~nm}$ pulses for the scavenger bunch. The wavelength is shorter in order to increase the cathode quantum efficiency, as the polarization is not required for the scavenger pulse.

\subsubsection{Damping Ring Spin Transport}

From the injector, the electron bunch passes into the North Damping Ring (NDR). To preserve the spin in the damping ring, the average electron spin must be vertical on injection. Part of the NLTR serves to precess the spin from its original longitudinal orientation to a horizontal direction perpendicular to the electron's path. A superconducting solenoid in the NLTR then precesses the spin to a vertical orientation.

\footnotetext{
†The pseudo-random number generator chosen for SLC is a 33-bit feedback shift register, deThe pseudo-random number generator chosen for SLC is a 33-bit
scribed in [89]. Some of its properties germane to SLC are given in [90].

${ }^{\hat{3}}$ The operating wavelength for the bulk of the 1993 run was $865 \mathrm{~nm}$, after being optinized from $850 \mathrm{~nm}$ at the very beginning of 1993 . The wavelength was re-tuned for the thinner strained cathode
} used in 1994-1995.

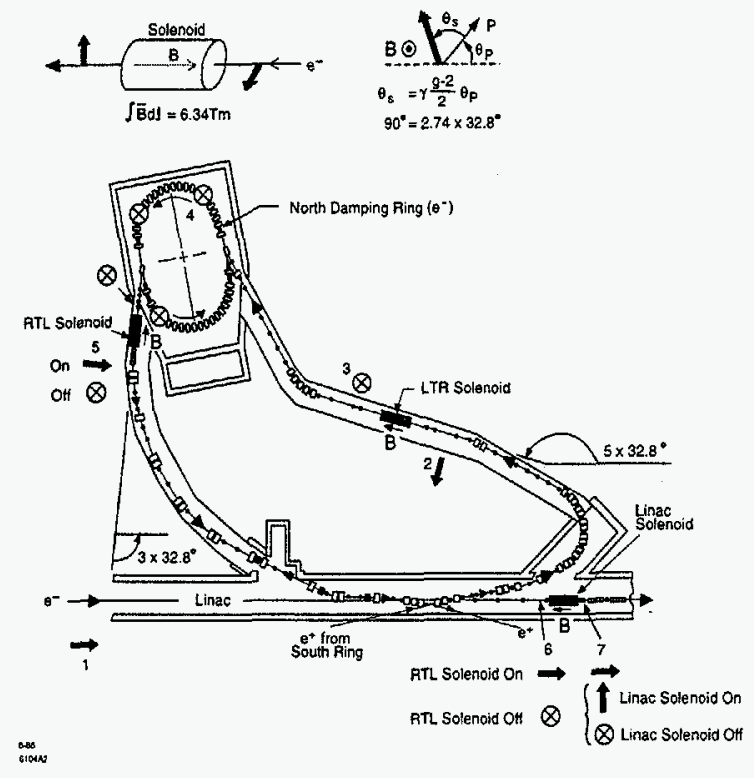

Figure 3.8: Spin transport in the North Damping Ring

The spin remains vertical in the damping ring, where the magnetic field is predominantly vertical. Any residual horizontal components to the spin quickly randomize away, as energy spread in the beam cause some electrons to precess faster than others. Upon extraction back into the Linac, the electron bunch passes through the NRTL (Ring-To-Linac) transfer line, which contains another spin-rotating solenoid. A third superconducting solenoid in the Linac shortly after the reinjection region provides a second degree of freedom in orienting the spin. The LTR solenoid's purpose is orient the spin vertically before the damping ring, so it cannot be used as a free parameter to orient the spin at the IP. The RTL and linac solenoids provide two independent adjustable spin parameters to orient the spin in any desired direction. This is necessary because the arc may introduce an arbitrary spin rotation, and the longitudinal component of the spin needs to be optimized at the IP for physics purposes. 


\subsubsection{Arc Spin Transport}

The transport of the spin vector in the North SLC arc has yielded some surprises and has supplied some valuable techniques for optimizing the operation of the accelerator. If the arc were built in a flat plane, then the spin would precess about an axis parallel to the dipole field in each magnet, and the magnitude of this precession would be given by

$$
\frac{d \theta_{\text {spin }}}{d \theta_{\text {bend }}}=\gamma \frac{g-2}{2}
$$

where $\gamma=E / m$ for the accelerated particles, and $(g-2) / 2 \approx 1.163 \times 10^{-3}$ is the anomalous magnetic moment of the electron. A pure Dirac particle's spin vector precesses at the same rate the velocity vector rotates, keeping them parallel. It is this extra precession that enables electron synchrotrons such as LEP to measure their energies with extreme precision by scanning the beam energy over a spin tune resonance, which quickly depolarizes the beam.

The SLC arcs are not constructed in a plane, so the bending dipole fields are not all vertical. Thus the electron spins do not precess about a single axis as they travel around the arc. Because rotations about different axes do not commute, the cumulative effect of the precession in each bend of the arc is not a simple function of the total bending angle, as is predicted for a flat arc. In particular, the dependence of the spin orientation at the end of the arc on the path the electron beam takes through the magnets is rather strong, and this was noticed in the $1992 \mathrm{run}$. Detailed computer simulations of the spin transport in the arc predict qualitatively, but not quantitatively, the properties of the precession in the arc. The reason the spin orientation is as sensitive as it is to the arc orbit is because the spin tune and the betatron tune of the arc are unintentionally matched, and with each oscillation in the orbit. spin precession offsets added constructively [91][92]

Fortunately, these properties of the arc may be used to the advantage of the experiment. Because the spin orientation at the IP depends on the orbit, an orbit may be chosen to maximize the longitudinal component of the spin there [91]. Such orbit modifications were named "spin bumps," because they involved introducing small deviations from the ideal orbit.
To verify that optimal spin bumps are chosen, the measured longitudinal component of the polarization can be compared against the known total magnitude of the spin. This upper limit to available longitudinal polarization may be determined in two ways. The longitudinal polarization at the IP is measured with the Compton polarimeter for a variety of orbits, and the polarization is found as a function of orbit parameters such as the position and angle of launch into the arc. A more rigorous method is to adjust the RTL and Linac spin rotators so that three mutually orthogonal polarization states are produced. The longitudinal component of each of these is measured at the IP, and the sum in quadrature of the longitudinal components from each of these measurements is the absolute magnitude of available polarization. If the total available polarization is known, then it is a quick procedure to adjust the orbit in the arc to maximize the longitudinal component of the spin at the IP.

An advantage of introducing spin bumps is that the energy dependence of the spin orientation may be minimized. Because the section of the North Arc before the reverse bend (see Figure 3.9) bends the beam in the opposite direction than the rest of the arc, the precessions in the two sections partially cancel. An orbit may be chosen to decrease the effective number of precession turns in the second portion of the arc, which dominates the total precession. This may be done by choosing an orbit that orients the spin parallel to the local dipole fields for a longer section of the arc. Reducing the effective number of precession turns is important for optimizing the polarization and keeping it stable. Spin diffusion in the arcs is proportional to the energy spread and the number of effective turns, so reducing both improves the polarization.

Another benefit of being able to optimize the spin orientation using spin bumps in the arc is that the spin rotators in the RTL and the Linac may be switched off (the-LTR rotator still has to be on in order to preserve polarization transmission through the NDR). Use of the RTL and Linac solenoids interferes with running flat beams, because they couple horizontal and vertical orbit deviations. Running the LTR solenoid is not a problem because the beams are flattened by the damping rings. Flat beams were commissioned in April of 1993 after the procedure for optimizing spin bumps became routine [78]. The final focus optics were originally optimized for 


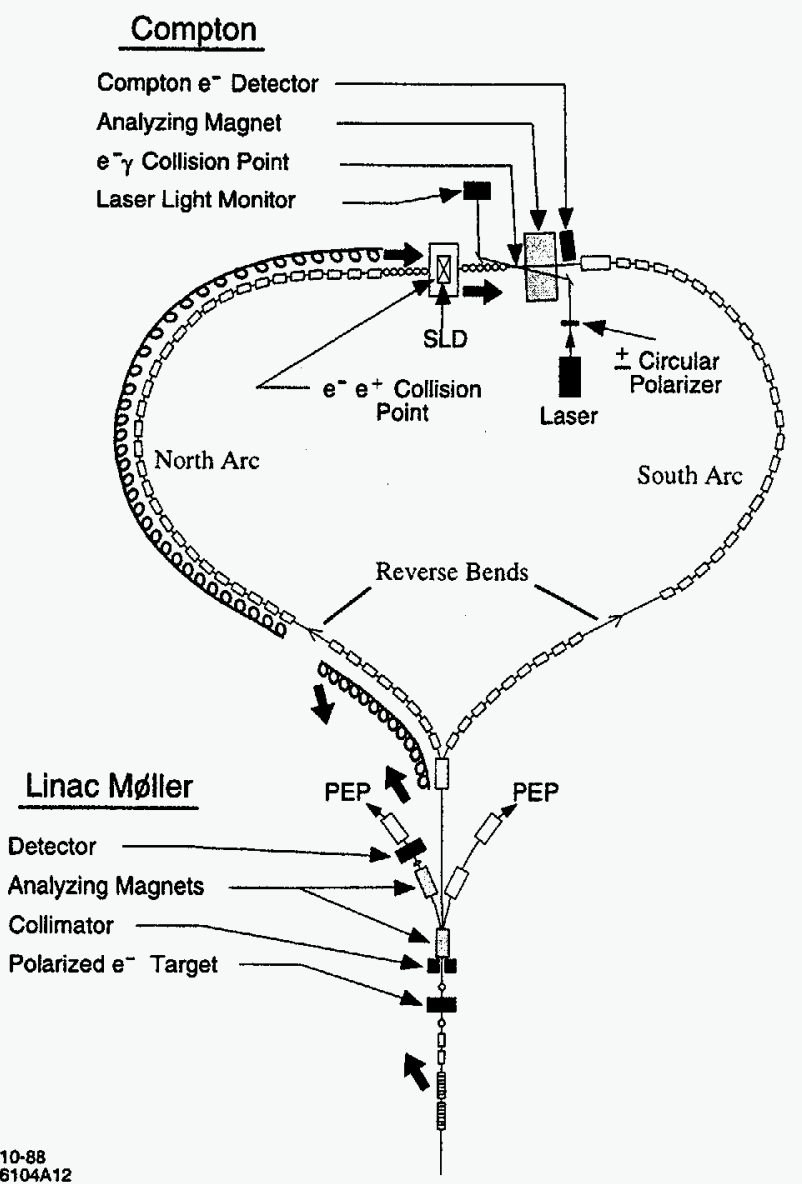

Figure 3.9: Spin transport and polarimeters at SLC. round beams, and reduced the aspect ratio of the flat beams. An upgrade to the optics [83], installed for 1994-1995 running, reduces the chromatic distortions and better preserves the aspect ratio.

\subsection{Spin Measurement}

The polarization dependence of the cross sections of $e^{+} e^{-} \rightarrow Z^{\circ} \rightarrow f \bar{f}$ arises in this experiment entirely from the longitudinal component of the electron spin. It is important to measure this quantity as close to the interaction region as possible to reduce systematic error on asymmetry measurements, and to measure it continuously; so that a luminosity-weighted average may be performed.

To measure the polarization accurately enough for the SLD $A_{L R}$ measurement, and to provide cross-checks, several polarimeters were put to use. A Compton polarimeter [93] provided the main measurement of the polarization during the run. A Møller polarimeter at the end of the Linac was used at selected times as a cross-check. In addition, a Mott polarimeter was used to test the cathode polarization, and that polarization was cross-checked independently by several polarimeters at other institutions. To check the polarization calibration of the Compton polarimeter, a second Møller polarimeter was installed in the electron extraction line at the end of the 1993 run, and also run briefly at the beginning of the 1994-1995 run.

\subsubsection{Compton Polarimeter}

The Compton polarimeter brings circularly polarized photons into collision with the SLC electron beam and measures the rate of Compton scattered electrons as a function of their energy. The electron beam arrives with randomly alternating helicity, and the helicity of the photons may also be chosen on a pulse-to-pulse basis. By comparing the asymmetries of the scattering rates in each of the four spin combinations, the polarization of the left-handed and right-handed electron bunches may be measured independently with high precision. 


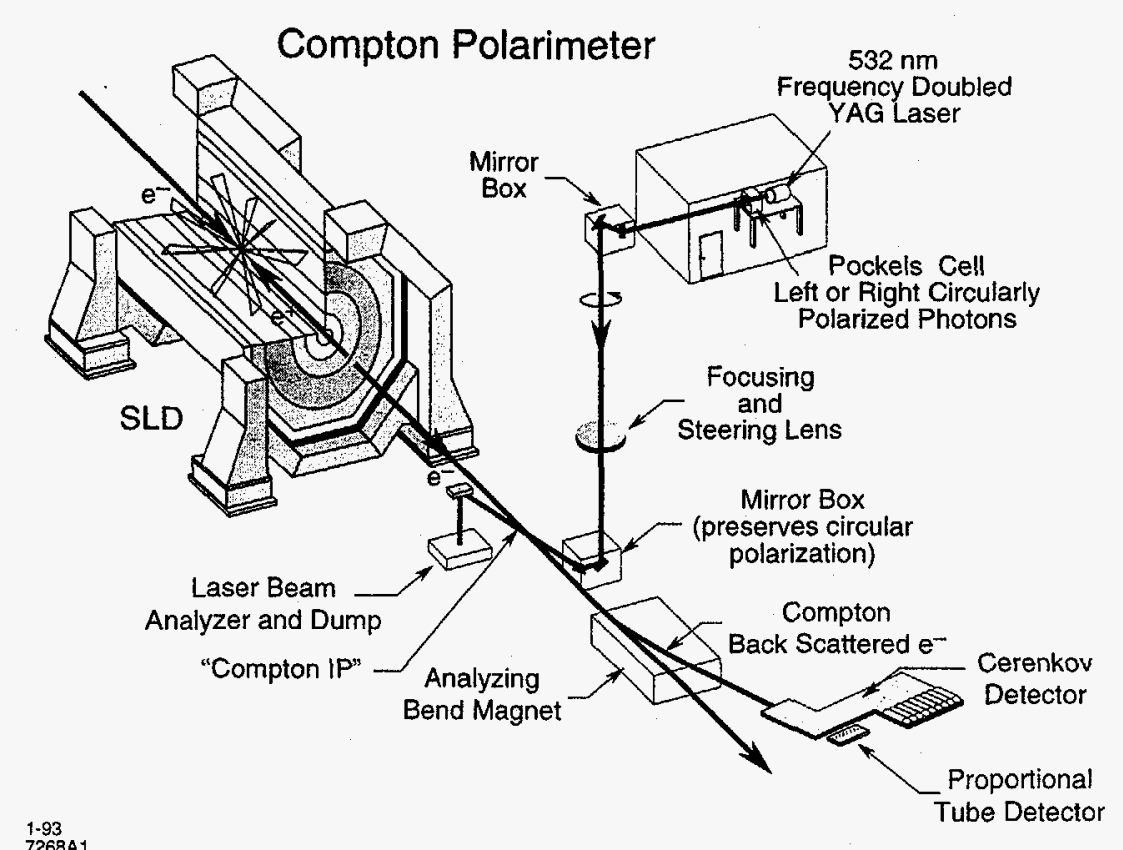

Figure 3.10: The Compton Polarimeter in the South Final Focus

\section{Compton Layout}

The Compton polarimeter is designed to measure the electron beam polarization with a minimum of bias, and to allow what biases there are to be measured. The polarimeter consists of a laser, polarizing optics, a beam transport tube, analyzing optics, an energy-analyzing bend magnet, and a detector for scattered high-energy electrons. In contrast to polarimeters at storage rings, the scattered photons are not used in the measurement.

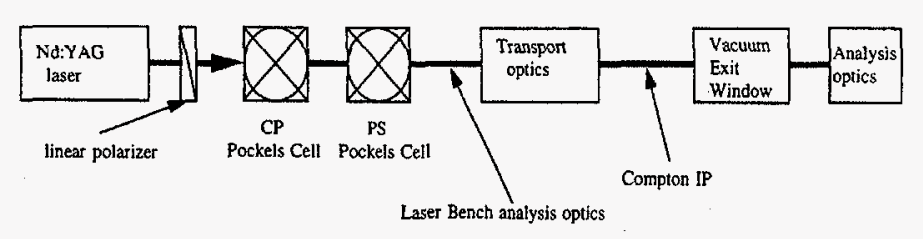

Figure 3.11: Schematic diagram of the Compton polarimeter optical layout.

\section{Compton Laser}

A Q-switched Nd-YAG laser, frequency-doubled to a wavelength of $532 \mathrm{~nm}(2.33$ $\mathrm{eV} /$ photon, green), supplies the light for the Compton measurement. To obtain adequate scattering, the laser must produce a short, intense pulse - long pulses would not fully overlap with the electron beam if the crossing angle is finite, and the scattering must be localized to a region of the electron beampipe free of bending magnetic fields. The energy delivered to the Compton interaction region per pulse was $50 \mathrm{~mJ}$ and the pulse duration was $7 \mathrm{~ns}$. The laser only fired on a fraction of available electron beam-crossings; the electron bunches arrive at $120 \mathrm{~Hz}$, and the laser pulsed on average at $11 \mathrm{~Hz}$ (1993) and $17 \mathrm{~Hz}(1994-5)$. The frequency was chosen to keep the laser pulses from being synchronized with periodic behavior of the collider".

\section{Polarizing Optics}

The most important optical elements immediately after the laser are a linear polarizer and two Pockels cells [95]. The first Pockels cell's optical axis is oriented at $45^{\circ}$ to the plane of linear polarization of the incoming light, and can circularly polarize the laser beam with either handedness. Typical voltages applied to the first Pockels cell are $\pm 1200 \mathrm{~V}$. The optimal voltages for the two helicities are not exactly negatives of one another due to residual optical activity in the Pockels cell crystal even at zero applied voltage. The helicity is chosen pseudorandomly to explore all spin combinations with

\footnotetext{
"This strategy was not entirely successful in 1994, as an energy feedforward device in the north damping ring caused every seventh SLC pulse to be off-energy, and the Compton laser pulsed every seventh SLC cycle. Fortunately, this did not cause a significant problem for analyses because the relative phase of the off-energy pulse constantly drifted. See Reference [94] for details.
} 
the electrons with equal weight.

The second Pockels cell's optical axis is aligned at $90^{\circ}$ to the first one, and together with the first cell, allows for the separate manipulation of the Stokes parameters of the laser lightll. In particular, components of the light transport system can incur phase shifts in the left- and right-handed components of the light, transforming circularly polarized light into elliptical light by the time it gets to the Compton interaction region. These phase shifts can be measured by opening the vacuum chamber of the electron beampipe and measuring the polarization components at the interaction region itself. Care must be taken because mechanical stress on vacuum windows may affect the phase shifts they induce.

The transport line phase shifts are also affected by the temperature of the optical elements and their coatings, and by their alignment. It is necessary to measure these phase shifts continuously throughout the run to minimize systematic uncertainties. A procedure to scan the voltages of the two Pockels cells, described later, was instituted to perform this task.

\section{Transport Line}

The laser light, after being appropriately polarized, is then guided from the optical bench to the Compton interaction region in the SLC tunnel. The total length of the laser transport system is 40 meters. Four compound mirrors are needed to bring the light from the laser shack to the SLC beampipe. Each compound mirror consists of two matched mirrors in order to compensate for phase shifts incurred by reflection and passage through optical coatings. A remotely movable lens in the transport system serves the dual purposes of focusing the light in the interaction region and steering the laser beam into collision if the alignment is disturbed, usually by diurnal temperature variations. The transport tube itself was evacuated for the majority of the 1993 run. but filled with helium at low pressure later in the run to help control damage to the optics, which was suspected to arise from electrical breakdown in the residual gas

\footnotetext{
"There are four Stokes parameters, but two degrees of freedom are taken by the total light intensity and the fraction of unpolarized light, leaving two degrees of freedom for the polarization,
} which may be manipulated by the voltages on two Pockels cells. near the optical elements. After exiting the laser transport tube, the light passes through a vacuum-tight quartz window to the SLC vacuum chamber. The crossing angle of the laser light and electron beam is $10 \mathrm{mrad}$, and the Compton interaction point is 32 meters south of the SLC interaction point.

\section{Analysis Box}

After colliding with the electron beam, the remaining laser light is brought out of the SLC beampipe through another quartz window and its polarization is measured by breaking it into its left-and right- handed components with a quarter-wave plate and a calcite prism [95]. The measurement of the light polarization after the Compton interaction point is critical for determining the unpolarized fraction of the laser light. By adjusting the voltages on the two Pockels cells, one may explore all possible polarization states at the end of the light transport system. If the maximum attainable polarization in the analysis optics falls short of unity, then a component of unpolarized light may be inferred. For the 1993 run, the unpolarized fraction has been determined in this manner to be less than $1.0 \%$, and for the $1994-5$ run, it is less than $0.6 \%$.

\section{Compton Spectrometer and Detectors}

The electrons which undergo Compton scattering are deflected away from the beamline by two arc bending magnets. The integral of $B \cdot d l$ in these magnets is 1.67 kGauss-meters. A detector is placed $355 \mathrm{~cm}$ downstream from the bend point of the spectrometer, covering the region from $\simeq 5 \mathrm{~cm}$ from the beam to $\simeq 14 \mathrm{~cm}$. This latter coverage can be adjusted by pivoting the detector table, maintaining the projectivity of the Cherenkov radiator tubes to the Compton IP. The detector assembly consists of one inch of lead as a shower preradiator and nine Cherenkov radiator tubes filled with a propane for the majority of the 1994-1995 run ( $\beta$-butylene was used as a radiator previously, with the switch to propane made in order to reduce buildup of residue on the optical surfaces). Behind the tubes, but offset away from the beamline so as to reduce background from the showering electrons, are Hamamatsu R1398 phototubes to record Cherenkov radiation. Set behind the Cherenkov radiator tubes is an array 


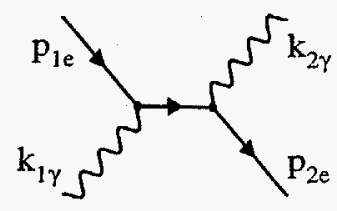

s-channel

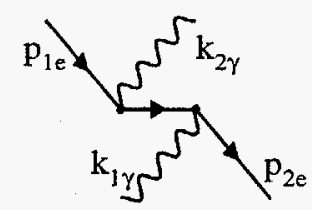

u-channel
Figure 3.12: Lowest-order contributions to Compton scattering. In the totally backscattered case at high energy, the s-channel process is highly suppressed. The kinematic variables labeled are SLC laboratory frame variables.

of 16 proportional tubes in a block of tungsten. The signal from the proportional tubes is correlated with that from the Cherenkov tubes on a pulse-to-pulse basis, so the measured polarization is expected to be the same. The purpose of haring two detectors is to provide cross-checks on them both.

\section{Compton Measurement}

The differential cross-section for Compton scattering is given to lowest order by computing the two diagrams of Figure 3.12. For unpolarized scattering, the differential cross-section is given by the Klein-Nishina formula [96] in the rest frame of the electron:

$$
\frac{d \sigma}{d \cos \theta}=\frac{\pi \alpha^{2}}{m_{\epsilon}^{2}}\left(\frac{k^{\prime}}{k}\right)^{2}\left[\frac{k^{\prime}}{k}+\frac{k}{k^{\prime}}-\sin ^{2} \theta\right],
$$

where $k$ is the initial energy of the photon, $k^{\prime}$ is the final energy, and $\theta$ is scattering angle of the photon. The electromagnetic coupling constant $\alpha$ takes nearly its $Q^{2}=0$ value due to the small center-of-mass energy of the $e-\gamma$ collision. The scattering angle and energies are related by the relativistic kinematic constraint:

$$
k^{\prime}=\frac{k}{1+\frac{k}{m_{e}}(1-\cos \theta)} .
$$

Boosting this result into the SLC frame, one obtains:

$$
\frac{d \sigma}{d E_{2}}=\frac{\pi \alpha^{2}}{m k^{2}}\left(\frac{k^{\prime}}{k}+\frac{k}{k^{\prime}}+1-\left(1+\frac{m_{e}}{k}-\frac{m_{e}}{k^{\prime}}\right)^{2}\right) .
$$

Expressed in terms of SLC laboratory frame variables, $k$ and $k^{\prime}$ become

$$
k=\frac{E_{1 \gamma} E_{1}}{2 m_{e}},
$$

and

$$
k^{\prime}=\frac{E_{1 \gamma} E_{1}}{2 m_{e}}\left(1-\frac{\left(E_{1}-E_{2}\right)}{E_{1}}\right)
$$

where $E_{1}$ is the electron beam energy $(45.6 \mathrm{GeV}), E_{1 \gamma}$ is the incident laser beam energy $(2.33 \mathrm{eV})$, and $E_{2}$ is the scattered electron's energy.

Equations 3.3 and 3.5 are averages over the incident electron and photon spin states. The full polarization-dependent formula can be expressed as two terms - one symmetric in the initial longitudinal spin state configuration, and one antisymmetric in it. It is the product of the electron and photon spins that appears because the process obeys parity symmetry. The two $J=3 / 2$ initial state combinations have the same scattering cross section, which is larger in the backscattered case than the cross section of the two $J=1 / 2$ initial states.

$$
\frac{d \sigma}{d E_{2}}=\frac{d \sigma_{\text {unpolarized }}}{d E_{2}}\left[1+\mathcal{P}_{e} \mathcal{P}_{\gamma} A_{\text {Compton }}\left(E_{2}\right)\right]
$$

The quantity $A_{\text {Compton }}$ is defined to be the "analyzing power" for Compton scattering, the dilution of the polarization asymmetry when the asymmetry of the scattering is formed:

$$
A_{\text {Compton }} \mathcal{P}_{\mathrm{e}} \mathcal{P}_{\gamma}=\frac{\sigma_{3 / 2}(E)-\sigma_{1 / 2}(E)}{\sigma_{3 / 2}(E)+\sigma_{1 / 2}(E)}
$$

This asymmetry can be expressed in terms of SLC variables [97]:

$$
A_{C o m p t o n}=\frac{\left(A B^{3}-A^{3} B\right)+m_{e}^{2}\left(A B^{2}+A^{2} B-A^{3}-B^{3}\right)}{\left(A B^{3}+A^{3} B\right)+m_{e}^{2}\left(2 A^{2} B-2 A B^{2}\right)+m_{c}^{4}\left(A^{2}+B^{2}-2 A B\right)},
$$

where

$$
A=-E_{1} E_{1 \gamma}-\left|p_{1}\right| E_{1 \gamma}=-p_{1 e}^{\mu} k_{1 \gamma \mu}
$$

is the dot product of the initial electron's four-momentum into the initial photon's, and

$$
B=-E_{2} E_{1 \gamma}-\left|p_{2}\right| E_{1 \gamma} \cos \theta_{p}=-p_{2 e}^{\mu} k_{1 \gamma \mu}
$$




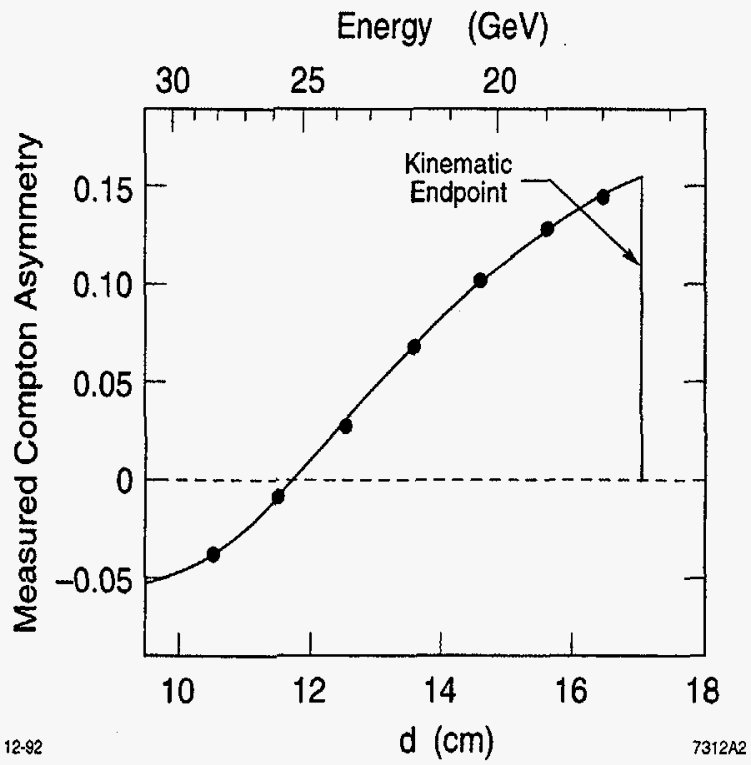

Figure 3.13: The asymmetry between the $J=3 / 2$ and $J=1 / 2$ states of Compton scattering as a function of the energy lost by the electron, compared against the measured asymmetry for $P_{e}=63 \%$. The distance scale is the defiection of the scattered electrons at the front of the Cherenkov detector.

is the dot product of the outgoing electron's four-momentum and the initial photon's. The electron's scattering angle $\theta_{p}$ is given by the following hinematic constraint:

$$
\cos \theta_{p}=\frac{E_{1} E_{2}+E_{1 \gamma} E_{2}-E_{1 \gamma} E_{1}-E_{1 \gamma}\left|p_{1}\right|-m_{e}^{2}}{\left|p_{2}\right|\left(\left|p_{1}\right|-E_{1 \gamma}\right)}
$$

where $\left|p_{1}\right|$ and $\left|p_{2}\right|$ are the momenta of the incoming and outgoing electron, respectively.

Because the Compton polarimeter detects only the scattered electrons, a higherorder QED calculation needs to be performed in terms of the energy of the scattered electron. Such a calculation has been performed to one loop, including bremsstrahlung [97]. The derived $A_{\text {Compton }}$ is shown in Figure 3.13.

To measure the asymmetry as a function of energy, the signal in each Cherenkov channel is accumulated separately for each combination of electron and laser helicity states, and also for those beam crossings on which the laser did not fire. Let $s_{j k}^{i}$ be the average of the signal in channel $i$ when the electrons are in state $j=(L, R)$ and the photons are in state $k=(L, R, o f f)$, and let $\sigma_{j k}^{i}$ be the statistical error on $s_{j k}^{i}$. Then the raw asymmetry for the left-handed electron beam is given for each channel by

$$
A_{\text {raw }}^{L, i}=\frac{s_{L R}^{i}-s_{L L}^{i}}{s_{L R}^{i}+s_{L L}^{i}-2 s_{L o f f}^{i}}
$$

and for the right-handed electron beam it is

$$
A_{r a w}^{R, i}=\frac{s_{R R}^{i}-s_{R L}^{i}}{s_{R R}^{i}+s_{R L}^{i}-2 s_{R o f f}^{i}}
$$

In this way the polarization of both helicity states of the electron beam may be measured independently, even if the backgrounds and beam currents are systematically: different for the two beam states. The statistical error on $A_{r a w}^{L, i}$ is given by

$$
\delta A_{\text {raw }}^{L, i}=\frac{2\left(\left(s_{L L}^{i}-s_{L o f f}^{i}\right)^{2}\left(\sigma_{L R}^{i}\right)^{2}+\left(s_{L R}^{i}-s_{L o f f}^{i}\right)^{2}\left(\sigma_{L L}^{i}\right)^{2}+\left(s_{L R}^{i}-s_{L L}^{i}\right)^{2}\left(\sigma_{L o f f}^{i}\right)^{2}\right)^{1 / 2}}{s_{L R}^{i}+s_{L L}^{I}-2 s_{L o f f}^{i}}
$$

with a similar expression holding for the right-handed case.

Two features of Compton scattering serve to provide absolute reference points in the scattering spectrum. The first of these is the kinematic scattering limit. Electrons of energy $E_{1}$ may lose a maximum energy of

$$
\Delta(E)_{\max }=E_{1}\left[1-\left(1-\frac{4 E_{1} E_{1 \gamma}}{m_{e}^{2}}\right)^{-1}\right]
$$

when they strike photons of energy $E_{1 \gamma}$, corresponding to backscattering in the Compton $C M$ frame. For $E_{1}=45.6 \mathrm{GeV}$ and $E_{1 \gamma}=2.33 \mathrm{eV}$, backscattered electrons have an energy of $17.37 \mathrm{GeV}$ in the laboratory frame. These electrons are deflected 52.7 mrad by the spectrometer magnets and are intercepted by the Compton detector 35.5 $\mathrm{cm}$ downstream from the bend point. Unscattered electrons are bent by 20 mrad, so the displacement of fully backscattered electrons from the beampipe at the detector is $11.6 \mathrm{~cm}$. 
The other landmark on the Compton spectrum is the zero asymmetry point, corresponding to a scattering angle of $\pi / 2$ in the Compton CM frame. This corresponds to an angle of $36.2 \mathrm{mrad}$ after the bend spectrometer. Together, these two points on the Compton spectrum serve to locate its image on the Cherenkov tubes, and to determine its distance scale. The Cherenkov detector was positioned so that the kinematic endpoint lay in the acceptance of the seventh channel from the beampipe, while the zero asymmetry point lay in the acceptance of the second channel. Studies with the EGS shower simulation program [98] were done to evaluate the effects of signal sharing between channels and the resulting expected asymmetry fit to the data is shown in Figure 3.13.

The Compton measurements are statistically powerful because they sample a large fraction of the beam crossings from SLC. Only the signal from the channel closest to the Compton endpoint is used to minimize systematic errors. The signal in the other channels of the detector are correlated with one another not only because of the width of the electromagnetic showers, but because the luminosity of the $\epsilon-\gamma$ collision depends on the temporal details of the laser pulse, which fluctuate significantly from pulse to pulse.

\section{Systematic Errors}

\section{Linearity}

One of the systematic errors on the polarization measurement arises from nonlinearities in the responses of the detector, phototubes, and electronics. The emission of Cherenkov radiation in the detector is an inherently linear process - there is no saturation effect as additional shower electrons travel through the same radiator. The signal may be degraded by coatings and contamination on the reflective surfaces on the channel walls and mirrors, but this has no effect on the asymmetries. Phototubes, on the other hand, saturate easily and care must be taken to keep the voltage on the different stages from sagging under the load of a large signal. A special phototube base was designed [99] to improve linearity. The linearity of the digitizers was measured with an external pulse generator $[100]$.

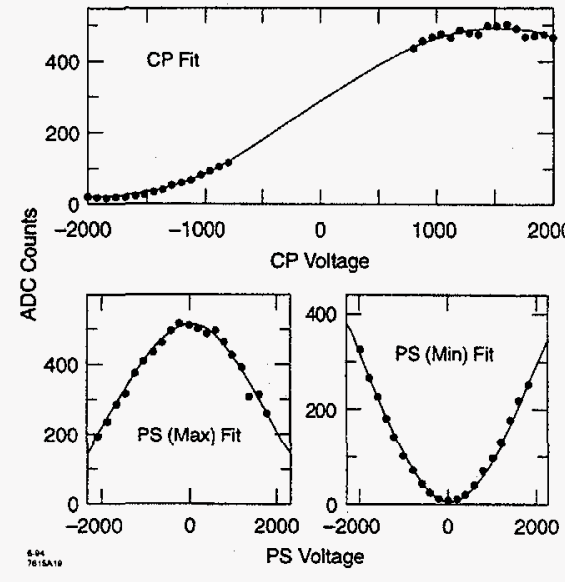

Figure 3.14: A scan over the voltages of the CP and PS Pockels cells to determine the quarter-wave voltage and the unpolarized fraction.

The linearity of the entire system may be measured in several ways. One is to adjust the voltage on a single phototube until it starts to saturate. The beam polarization is monitored by the other phototubes in the system and the test phototube's asymmetry is compared against the others. It is then estimated how far the normal operating point is from the nonlinear region.

A second method is to adjust the laser power until the signal gets large enough to affect the system's linearity. Because the phototube gains vary significantly, some channels will enter their nonlinear regions before others. Testing consistency between the polarizations derived from the raw asymmetries in each channel also provides an estimate of the reliability of the measurement. The estimated error from detector linearity for the 1994-1995 run is $0.5 \%$.

\section{Laser Polarization}

Another systematic error arises from the need to know the laser polarization to compute the electron polarization. Because the transport line contains many optical elements which can introduce relative phase shifts between the linear components 


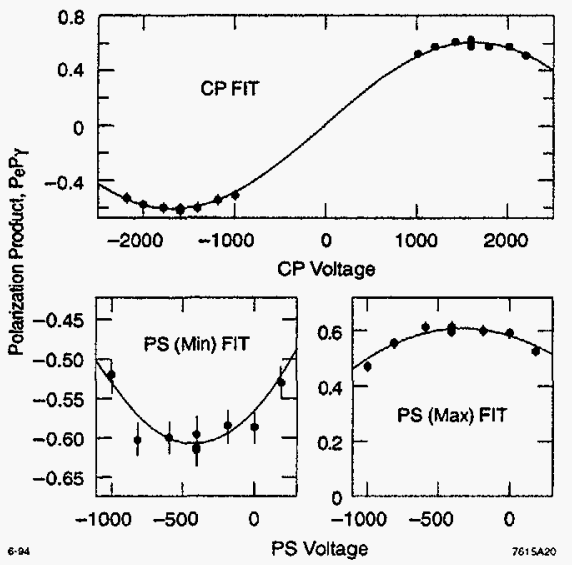

Figure 3.15: A scan over the voltages of the CP and PS Pockels cells to determine the optimal phase of the light to measure the electron beam polarization.

of the light, the absolute magnitude of the laser polarization at the scattering point must be treated carefully in order to reduce its uncertainty. The voltages on both Pockels cells are routinely scanned and the Compton asymmetry measured on each scan point. The asymmetry is then fit to a function sinusoidal in the voltages of both of the Pockels cells:

$$
P_{e}^{\text {meas }}=P_{e}^{0} \cos \left(V_{C P}-V_{C P}^{0}\right) \cos \left(V_{P S}-V_{P S}^{0}\right) .
$$

Here, $V_{C P}$ is the voltage on the circularly polarizing Pockels cell, and $V_{P S}$ is the voltage on the phase shift Pockels cell. The parameters $P_{\epsilon}^{0}, V_{C P}^{0}$, and $V_{P S}^{0}$ are allowed to float in the fit, and $P_{e}^{0}$ is the measured value of the polarization. A sample of a complete scan is shown in Figure 3.15. To determine the unpolarized fraction of laser light, every hour a fast scan over the two Pockels cell voltages was done and the photon polarization measured in the analysis box. A sample scan is shown in Figure 3.14 , where the unpolarized fraction is $<1 \%$. The total estimated systematic error on the electron polarization arising from uncertainties in the laser polarization is $0.2 \%(1994-1995)$ and $1.0 \%(1993)$.
To monitor the electron polarization during the run, the measurements are alternated between those near $V_{C P}=V_{C P}^{0}, V_{P S}=V_{P S}^{0}$, and measurements at other voltages. These measurements are less sensitive to fluctuations in the transport line's phase shift, owing to the zero derivative of the cosine function at its maximum, and are reported to the experiment and accelerator operators during the run.

\subsubsection{Møller Polarimeter}

Because the SLD measurement of $A_{L R}$ is the most precise single measurement of $\sin ^{2} \theta_{W}$, and because the main systematic error on that measurement arises from uncertainties in the electron polarization measurement, it was determined that a second measurement of the electron polarization needed to be done with a polarimeter with systematic uncertainties as independent as possible from those of the Compton polarimeter.

A second polarimeter [101], taking advantage of the unequal Møller cross-sections for aligned and antialigned incident and target electron spins, had already been installed in the SLAC beam switchyard for use with the fixed-target program. The target, a thin, magnetizable foil, is inserted in the beam in the switchyard. The dipole necessary for shunting electrons into the PEP injection line is then used as a momentum-analyzing magnet. A detector is then located at such a place that 46.6 $\mathrm{GeV}$ electrons scattering elastically on target electrons in the foil will be steered into it by the analyzing magnet. The detector consists of 64 silicon strips with a pitch of $600 \mu \mathrm{m}$, oriented perpendicular to the swath of electrons swept out by the analyzing magnet so as to form an energy spectrometer. Collimators and shields reduce backgrounds from electrons scattering on the nuclei in the target. The target is surrounded by three pairs of Helmholtz coils, which may magnetize the target in any direction. By using these three axes of target polarization, the three components of the beam polarization may be measured.

The scattering of high-energy polarized electrons and a stationary polarized electron target allows the measurement of the initial beam's polarization [101]. Typical targets are made of an iron-cobalt-vanadium alloy (Vanadium-Permendur: $49 \% \mathrm{Fe}$, $49 \% \mathrm{Co}$, and $2 \% \mathrm{~V}$ ). The observed asymmetry, however, is diluted by the fact that at 


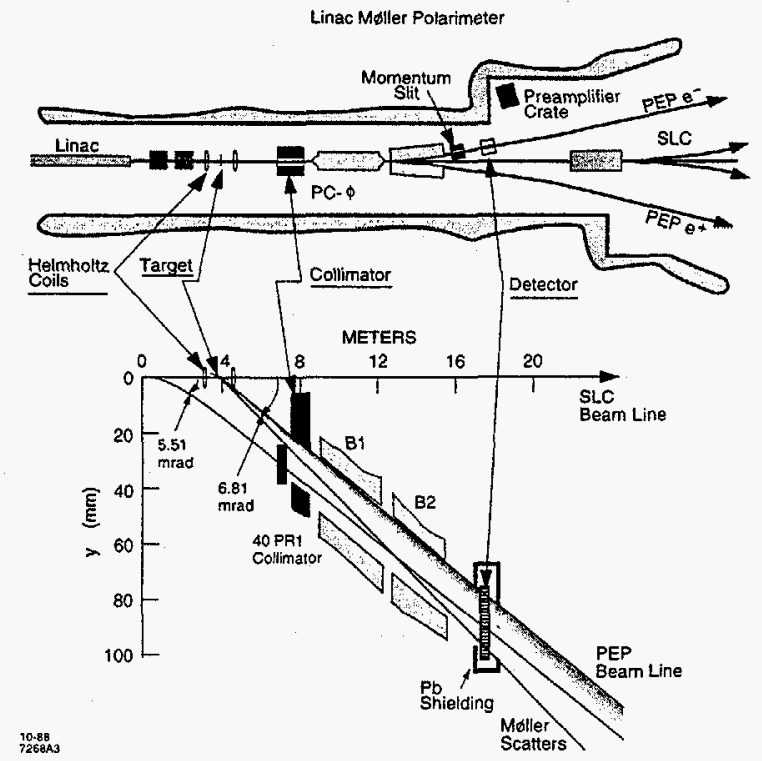

most one electron per atom participates in the magnetic ordering, while all electrons contribute to Møller scattering.

The analysis of Møller polarimeter data mainly consists of understanding the signal shape, estimating the background contribution, and fitting the asymmetry as a function of scattered electron energy. A naive fit of the data from this polarimeter is shown in Figure 3.16 and yields a polarization value of $(82.4 \pm 2.7) \%$, whereas the Compton measured $(65.7 \pm 0.9) \%$, after correcting for expected depolarization in the North SLC arc. It was originally believed that the arc depolarization was poorly modeled. When the energy spread was reduced to minimize spin diffusion, and the number of effective turns reduced, the discrepancy between the Compton and Linac Møller remained.

A more complete analysis [101] of the data from the Møller polarimeter was performed shortly after a paper by M. Levchuk [102] was found that described the effect of the motion of atomically bound electrons on the spectrum from a Møller polarimeter. The effect arises from the fact that the inner shell electrons in iron are unpolarized,
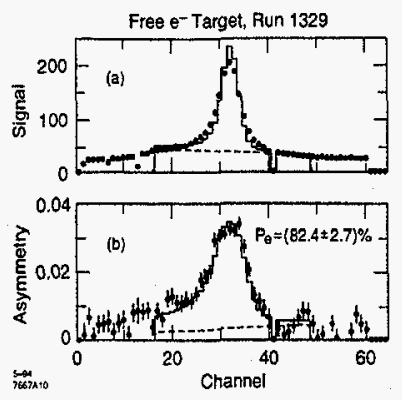

Figure 3.16: A fit to the measured signal and asymmetry (points) using the unbound electron hypothesis (histogram). Dashed lines indicate estimated background signal and asymmetry. From Reference [101].
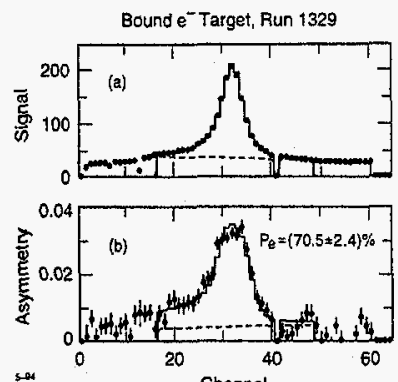

sostis

Figure 3.17: A fit to the measured signal and asymmetry (points) using the bound electron hypothesis (histogram). Dashed lines indicate estimated background signal and asymmetry. From Reference [101].

but they have a higher expectation value for their speed in the laboratory frame. While their energy is still much less than that of the incident beam, it greatly affects the energy in the collision's center of mass. This atomic motion serves to broaden the energy spectrum of the scattered electrons. Nonetheless, the very outermost electrons, which carry all of the atom's magnetization, have relatively little kinetic energy before they are struck. Their contribution to the energy spectrum of scattered electrons is narrower, and carries all of the asymmetry. If the analysis is performed assuming that the expectation value of the electron's spin is independent of its hinetic energy, then the extracted polarization may be a mismeasurement by as much as $15 \%$. A more complete fit is shown also in Figure 3.17, and the extracted polarization is (70.012.4)\%, consistent with the Compton measurement.

A third polarimeter was installed at the end of the 1993 SLD run as a further cross-check before the reanalysis of the Linac Møller data was performed. This polarimeter was another Møller installed in the south electron extraction line. In 1993, approximately $3 / 4$ of the scattered electrons were shadowed by a vacuum fitting and did not travel to the detector. It was left in place to be re-run at the beginning of 
the 1994 run with several different target materials to test the intra-atomic electron motion assumptions, and to remove the occlusions. Its polarization measurements are also consistent with those of the Compton.

The main systematic error in Møller polarimetry is the uncertainty on the polarization of the target. The magnetization of a thin foil in an applied field is expected to be non-uniform - the magnetization in the center of the target foil is expected to exceed that near the edges. The estimated error is roughly $4 \%$ of the measured value. Other errors arise from the assumptions made in the analysis of the asymmetry of the background. If a Møller scattered electron scatters again in the field of a nucleus of another target atom, it will contribute to another portion of the energy spectrum, carrying its original asymmetry with it. This effect can be mitigated by using a thinner target foil. The total systematic error from Møller polarimetry is expected to be irreducible beyond $4 \%$, and therefore no plans are made to use it in the future as a measurement or as a cross-check.

As a further cross-check on the absolute scale of the electron beam polarization. a sample of unstrained cathode material was measured in the laboratory, and similar samples cut from the same wafer were sent to Rice University and the University of California, Irvine, where existing Mott polarimeters, with a nominal $1 \%$ precision, measured polarizations consistent with those measured in the SLC gun cathode test laboratory. This test bench measured the polarization of the strained cathode used in the 1993 run to be $65 \%$, also confirming the scale of the Compton polarimeter. Not all of the data were conclusive, though - samples tested at Nagoya and also the PEGGY polarimeter [103] at SLAC reported higher polarizations than those measured on the cathode test bench.

\subsubsection{Energy Spectrometer}

The WIre Synchrotron Radiation Detector (WISRD) performs the absolute energy measurement for the SLD experiments [104]. It is located in the electron extraction line shortly before the beam dump. A second unit is located in the corresponding location of the positron extraction line. Each spectrometer consists of two strong horizontal bend magnets and a weaker vertical bend magnet, shown in Figure 3.18.

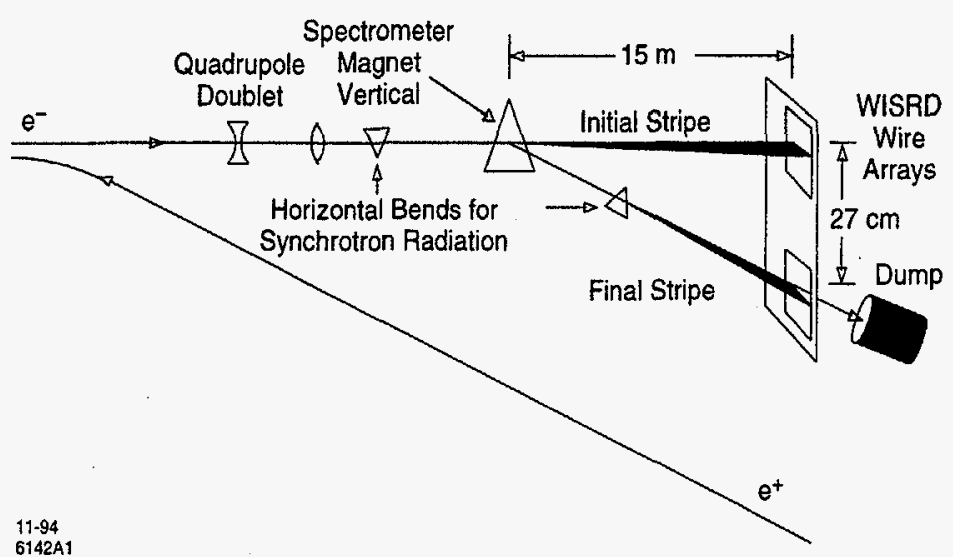

Figure 3.18: The Wire Imaging Synchrotron Radiation Detector (WISRD)

The first horizontal bend causes the electron beam to emit a horizontal swath of synchrotron radiation. It is immediately followed by a softer vertical bend, which deflects the beam downwards and serves as the energy analyzing magnet. The second hard horizontal bend deflects the electrons in the opposite direction as the first horizontal bend, and creates a second swath of synchrotron radiation. The vertical separation between these two swaths of radiation is inversely proportional to the energy of the beam. The difference in widths of the two swaths provides information about the energy spread of the beam.

The locations of the two swaths of synchrotron radiation are measured by proportional wire chambers located $15 \mathrm{~m}$ downstream from the magnets. The wire spacing is $100 \mu \mathrm{m}$ and they provide a resolution on the beam energy of $22 \mathrm{MeV}$. The energy spread resolution is less well known, and is best estimated with wire scans in high-dispersion locations in the arcs. Typical values of the energy spread are in the range of $50-100 \mathrm{MeV}$. Because the analysis presented in this thesis is only minimally dependent on the beam energies, errors from their measurement may be neglected. 
Table 3.2: SLC performance parameters for 1993-1995.

\begin{tabular}{|lcc|}
\hline Parameter & 1993 & $1994-1995$ \\
\hline$e^{+} /$bunch & $3.0 \times 10^{10}$ & $3.5 \times 10^{10}$ \\
$e^{-} /$bunch & $3.0 \times 10^{10}$ & $3.5 \times 10^{10}$ \\
$\sigma_{x}$ & $2.6 \mu \mathrm{m}$ & $2.3 \mu \mathrm{m}$ \\
$\sigma_{y}$ & $0.8 \mu \mathrm{m}$ & $0.5 \mu \mathrm{m}$ \\
$E_{C M}(\mathrm{GeV})$ & 91.26 & 91.26 \\
Energy Spread (\%) & 0.25 & 0.12 \\
$P_{e}$ & $63.0 \pm 1.1$ & $77.3 \pm 0.6$ \\
$\mathcal{L}\left(\mathrm{cm}^{2} \mathrm{~s}^{-1}\right)$ & $3.8 \times 10^{29}$ & $6 \times 10^{29}$ \\
Integrated $Z^{0}{ }^{\mathrm{s}}$ & $5.0 \times 10^{4}$ & $1.0 \times 10^{5}$ \\
\hline
\end{tabular}

\section{Chapter 4}

\section{Experimental Apparatus: SLD}

\subsection{Summary}

SLC's luminosity and polarization improve with every run. New cathodes for higher polarization, more feedback mechanisms for stability and increased collision time. rebuilt damping rings for higher current, and improved final focus optics for better chromatic behavior, have all contributed to SLC's recent successes. Many of the lessons learned at SLC will be valuable in designing and operating linear colliders of the future.

The Compton polarimeter is the highest-precision electron polarimeter operating at the beam energies of SLC, allowing precision tests of the Standard Model to be made.

\subsection{Luminosity Monitor}

The SLD luminosity monitor [106] consists of two distinct subsections: the Luminosity Monitor and Small Angle Tagger (LMSAT), which covers the angular region from 23 mrad from the electron beam to $68 \mathrm{mrad}$, and the Medium Angle Silicon Calorimeter (MASiC), which covers the region from $68 \mathrm{mrad}$ to $190 \mathrm{mrad}$.

The LMSAT is used primarily to detect $e^{+} e^{-}$pairs that have undergone Bhabha scattering at the SLC interaction point (IP). At low angles, the Bhabha cross-section is dominated by $t$-channel photon exchange and grows proportional to $\theta^{-4}$, where $\theta$ is the scattering angle. As Bhabha scattering is easily calculable to high precision 


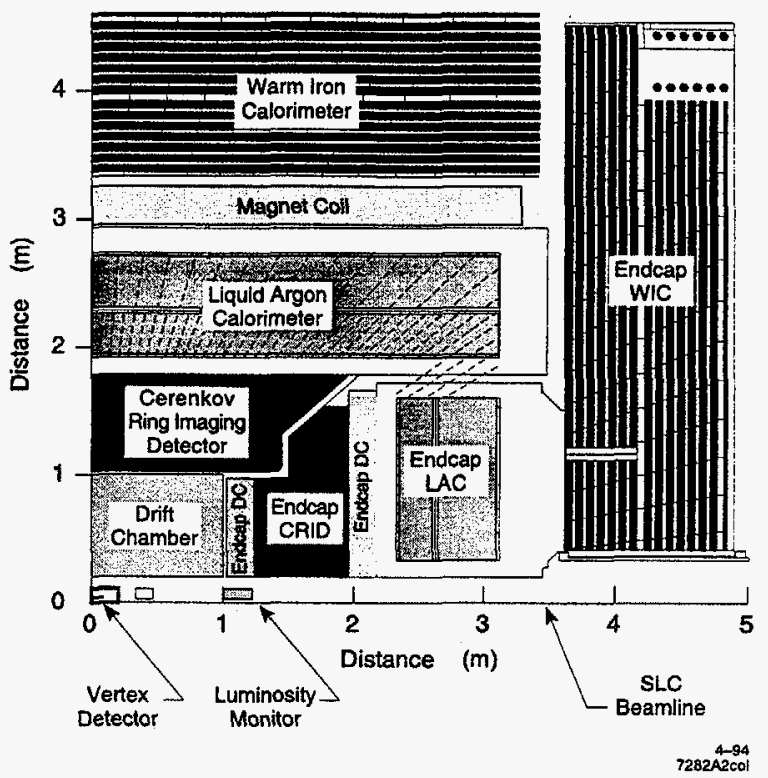

Figure 4.1: Quadrant view of SLD, showing the precision tracking, particle identification. calorimetry, and muon identification subsystems.

in QED, and because it occurs much more often than $Z^{0}$ production, it provides a precise measurement of the accelerator luminosity.

The LMSAT detectors are segmented into 32 sections in $\phi$ and 6 sections in $\theta$ (LMSAT). It is segmented into two longitudinal segments of 5.5 and 15.6 radiation lengths. The detectors are composed of tungsten radiator plates interleaved with silicon junction devices maintained in reverse bias. The energy resolution of the detector has been measured [107] to be $6 \%$ at $50 \mathrm{GeV}$.

\subsection{Tracking: Central Drift Chamber}

The bulk of the work of identifying and tracking the charged decay products of the $Z^{0}$ is performed by the Central Drift Chamber (CDC) [108]. Combined with the Vertex
Detector, it provides precision tracking necessary for heavy-quark physics at SLD.

\subsubsection{Mechanical Design}

The CDC consists of eighty layers of sense wires in a low-mass aluminum/Hexcel shell with an inner radius of $20 \mathrm{~cm}$ and an outer radius of $1 \mathrm{~m}$. The total length of the chamber is $2 \mathrm{~m}$, although dishing of the endplates causes the length of the wires to vary slightly from layer to layer. The $C D C$, as well as the particle identification system and the liquid argon calorimeter, lie inside a solenoidal magnet supplying a uniform $0.6 \mathrm{~T}$ field parallel to the bearn direction. The drift gas is a mixture of $75 \% \mathrm{CO}_{2}, 21 \%$ Argon, $4 \%$ Isobutane, and $0.2 \% \mathrm{H}_{2} \mathrm{O}$ and is maintained at a constant temperature of $20^{\circ} \mathrm{C}$ by a precisely controlled water cooling system. The pressure is allowed to equalize with the ambient atmospheric pressure. The drift velocity is 7.9 $\mu \mathrm{m} / \mathrm{ns}$ in the mean drift field of $0.9 \mathrm{kV} / \mathrm{cm}$. The estimated Lorentz angle is a very small $49 \mathrm{mrad}^{*}$.

The eighty layers of sense wires are grouped into ten "superlayers" of eight wires apiece ${ }^{\dagger}$. The first eight of these are arranged to be parallel to the beam ("axial layers"), while the next eight are tilted at an angle of $42 \mathrm{mrad}$ to provide stereo information (" $U$ layers"). The next eight are tilted also, but at an angle of $-42 \mathrm{mrad}$ (" $V$ " layers). The pattern is repeated, with the last eight wires forming an axial layer, and the overall layout is shown in Figure 4.2. The construction of the basic cell consists of a drift region with a nearly uniform field set up by the field and guard wires, and a nonlinear region between the guard wires and sense wires. Each cell measures roughly $6 \mathrm{~cm}$ wide by $5 \mathrm{~cm}$ high. A field map for a single cell is shown in Figure 4.3, illustrating the effects of the two ends of the cell on the electric field. The sense wires and guard wires of each cell are mounted on feedthroughs in a single block of Lexan on each end of the chamber. The field wires are supported by Celanex feedthroughs cold pressed into the chamber endplate. The sense wires are made of 25 $\mu \mathrm{m}$ gold-coated tungsten and are held at $100 \mathrm{gm}$ tension, while the guard and field

"It is the cosine of the Lorentz angle which corrects the drift distance, so the total effect is on the order of $0.1 \%$. Nonetheless, it is incorporated in both the simulation and reconstruction.

†There are actually ten "sense" wires in a superlayer, but the outer two are not digitized. The serve to enhance the feld uniformity for the other wires in the cell. 


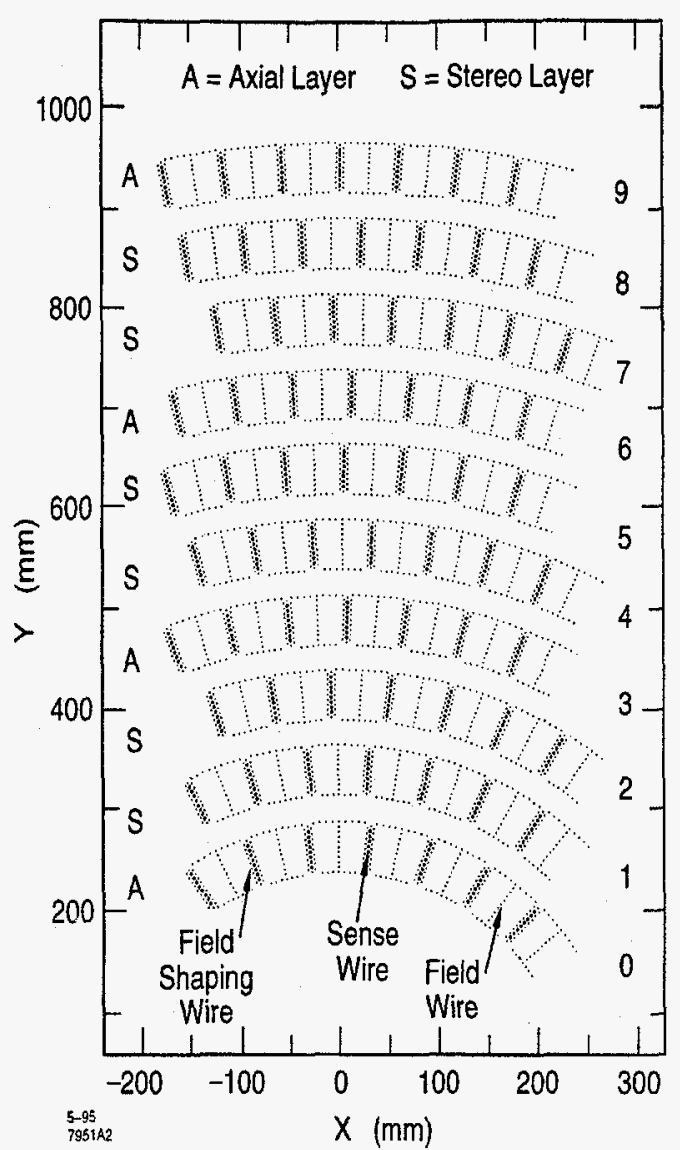

Figure 4.2: Cross-section of a portion of the $\mathrm{CDC}$ in the plane perpendicular to the beam axis: There are 540 projective drift cells in the chamber, each containing eight sense wires.

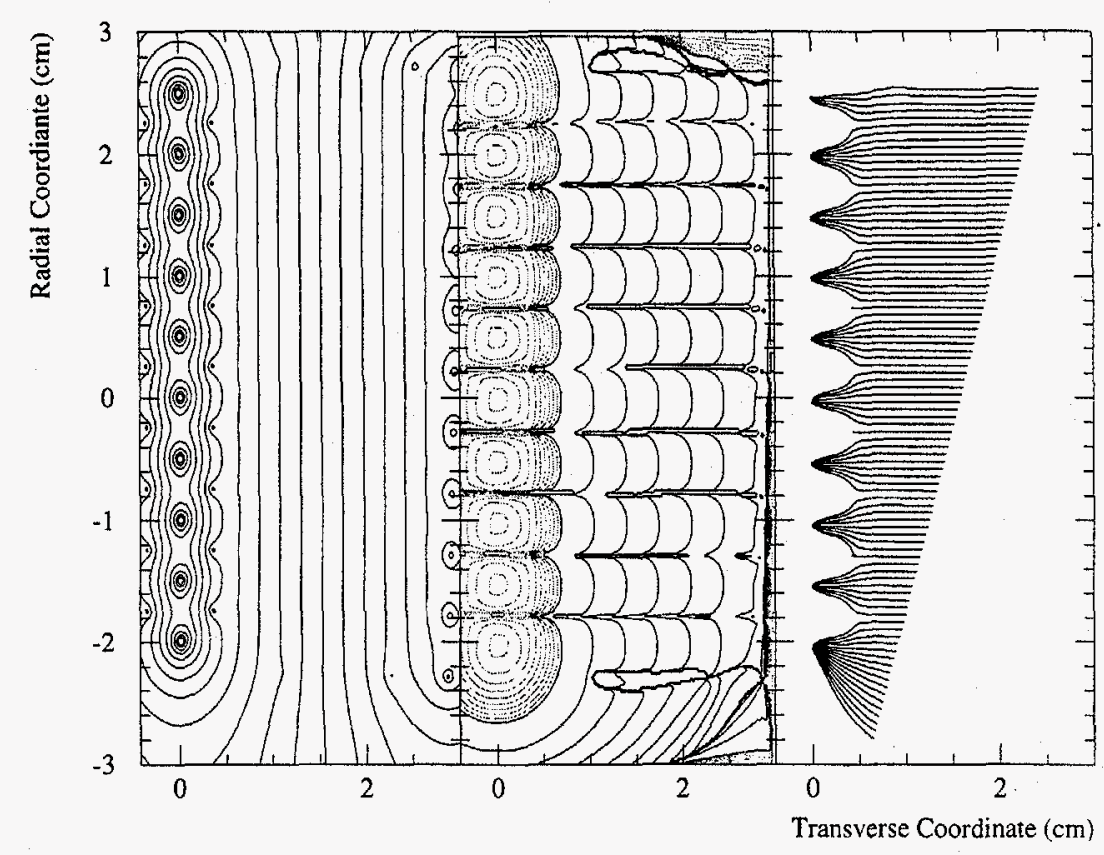

Figure 4.3: Diagrams showing the field in a half cell of the CDC. The leftmost figure displars the equipotentials of the electric field. Visible are four radially aligned layers of wires - the guard wires for the left half-cell, the sense wire plane, the right half-cell's guard wires, and the field wires to the right. The middle figure shows the drift-time isochrones for the same region. Isochrones near the sense wires are shown with $1 / 10$ the spacing as those outside. The rightmost figure shows the drift paths of representative electrons in the field of the drift cell for a sample track. The top and bottom sense wires are not read out. 
wires are made of $150 \mu \mathrm{m}$ gold-coated aluminum and held at $500 \mathrm{gm}$ of tension for the guard wires and $400 \mathrm{gm}$ of tension for the field wires.

Electrical pulses on the sense wires are read out on both ends by high-speed sampling electronics mounted directly on the endplates of the chamber. Signals are sampled at $119 \mathrm{MHz}$ and held in Hybrid Analog Memory Units (HAMUs), and digitized after the trigger decision has been made. Because the digitizers are mounted on the endplates, only digital information needs to travel to the data assembly electronics. Fiber optic transmission cables were chosen so as not to induce or receive electrical noise or create unintentional ground loops, and to reduce the total mass of cables on the detector.

Digitized signal values are then analyzed online by Waveform Sampling Modules (WSMs), located in Fastbus racks atop the detector. These modules apply a piecewise linear calibration correction to the incoming signals on a wire-by-wire basis. Pulses are found in the digitized waveforms by applying thresholds to the pulse height above baseline and the derivative of the pulse shape [109]. The found pulses are then analyzed to extract the pulse beginning time, end time, pulse height, width, and total charge. The signals on each end of the wire are analyzed separately for all wires on which a pulse is found. It is these quantities which are written to the SLD raw data tape in order to save space. A small fraction of randomly selected raw pulse data is also written for diagnostic purposes.

\subsubsection{CDC Reconstruction}

Tracks are found and fit in four stages - raw hit filtering, vector hit finding, pattern recognition, and track fitting. The first of these stages combines information for each pulse from both ends of the sense wire on which it arrived. The double-ended readout enables the position along the wire for that pulse to be estimated by calculating the charge division asymmetry for that pulse. The resolution on this estimate is approximately $2 \%$ of the wire length. Simple cuts are applied to the raw data pulses to remove common noise sources, such as synchrotron radiation and beam halo directly striking the amplifiers and HAMUs on the detector. These pulses are characterized by proximity in time to the beam crossing (no drift through the gas is involved), and small pulse height and total charge.

The second stage of reconstruction combines hits within superlayers which lie on straight lines or origin-constrained circles to form vector hits (VHs), which contain position and direction information for the track segments to which they correspond. Hit positions are then corrected for the effects of the relative angle between the track and the sense wire plane. The $z$ location of each vector hit is estimated from chargedivision information. The vector hit finder is described in more detail in Appendix C.

Pattern recognition [110] proceeds by analyzing the input VHs to form candidate tracks. Combinations of VHs on the axial layers of the chamber are formed by fitting them to circles. Discrimination between axial combinations is provided by attempting to add VHs from stereo layers. Because the $z$ resolution of each VH is poor, and in some cases nonexistent due to malfunctioning electronics on one end of the chamber for a group of cells, it is easy to assign nearly any plausible stereo VH to any axial combination. The $z$ position and dip angle provide enough freedom to match the position and angle of the VH to nearly any circle desired. The discriminating power comes from requiring that the derived $z$ positions and dip angles be consistent for all stereo VH's added to the axial combination. Axial combinations with 10 consistent. VH's are considered first, and the one with the best $\chi^{2}$ is taken as a candidate track. Its VHs are removed from the input list and the process is repeated. Once all possible 10-VH tracks are exhausted, the algorithm searches for 9-VH tracks, and continues until all tracks with at least $3 \mathrm{VHs}$ are found. No origin constraining bias is applied anywhere in the algorithm.

The fourth stage of track reconstruction is performed by the track fitter. The fitter starts with the estimated track parameters from the pattern recognition. It then swims a helical trajectory through the detector material, modifying it to take into-account the effects of energy loss, multiple scattering, and local variations in the magnetic field. A $\chi^{2}$ is formed, and its derivatives with respect to each of the five track parameters (the curvature, the dip angle, the azimuthal angle, and two position parameters) are estimated. In addition, the matrix of second derivatives, $\partial^{2} \chi^{2} / \partial x_{i} \partial x_{j}$ where $x_{i}$ and $x_{j}$ are the $i^{t h}$ and $j^{\text {th }}$ track parameters, is formed. The local minimum of $\chi^{2}$ is sought iteratively using these derivatives. On each iteration, 
the fitter is allowed to add or delete hits to improve the hit finding rate and $\chi^{2}$. When the iterations have converged, the matrix of second derivatives is inverted to form the error matrix for the track parameters.

\subsubsection{CDC Performance}

The performance of the CDC is quantified by the fraction of tracks reconstructed, and how well those tracks' reconstructed parameters match the properties of the particles that created them.

\section{Tracking Efficiency}

The reconstruction efficiency can be estimated by comparing the number of tracks found in data against those found in a Monte Carlo simulation. The JETSET 7.4 generator [30], with SLD adjusted heavy flavor decays, described in Chapter 5, is used in this comparison.

Figure 4.4 shows the reconstructed track multiplicity for selected hadronic events in data and Monte Carlo, after track and event selection described in Section 6.2.3. The effect of this discrepancy in the observed number of tracks can also be seen in Figures 4.6 and 4.5 , and its effects on the analysis of this thesis will be discussed in Section 6.3 .

Comparisons of the number of tracks in data and Monte Carlo as functions of momentum and polar angle $(\cos \theta)$ appear in Figures 4.6 and 4.5. The observed momentum dependence of the discrepancy between data and Monte Carlo could be a mismodeling of the momentum spectrum of charged tracks from $Z^{0}$ decay, or it could be the result of finding extra "fake" tracks in the fast, collimated cores of jets, where confusion between hits on neighboring tracks is greatest.

A related quantity to the tracking efficiency is the hit-finding efficiency. Most tracks selected within the angular acceptance of the CDC are expected to have a hit in each layer of the chamber. Figure 4.8 is the hit-finding efficiency of tracks with expected hits in a wire layer, as a function of the layer. The slow dip towards the inner layers is caused by the finite two-hit resolution as the tracks become closer

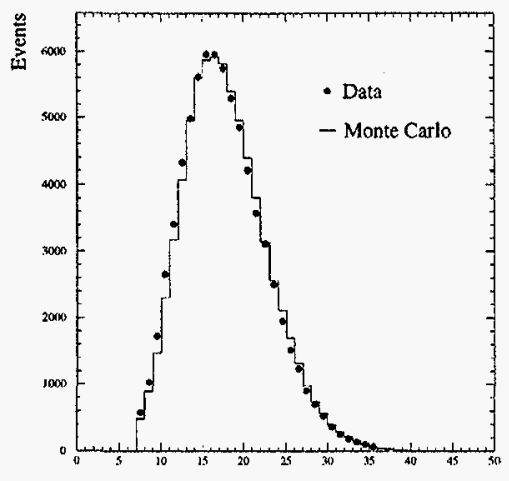

Track Multiplicity

Figure 4.4: Charged track multiplicity distribution for data and Monte Carlo. On average. the data has 0.4 tracks less than the Monte Carlo, which is a $2.3 \%$ discrepancy. The effect of this on the present analysis will be discussed in Chapters 6 and 7 .

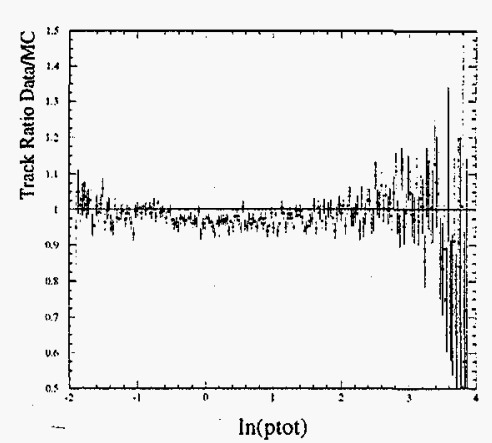

Figure 4.5: Ratio of tracks in Data to Monte Carlo as a function of $\ln \left(P_{t o t}\right)$, showing an overall inefficiency, but also an excess at high momentum.

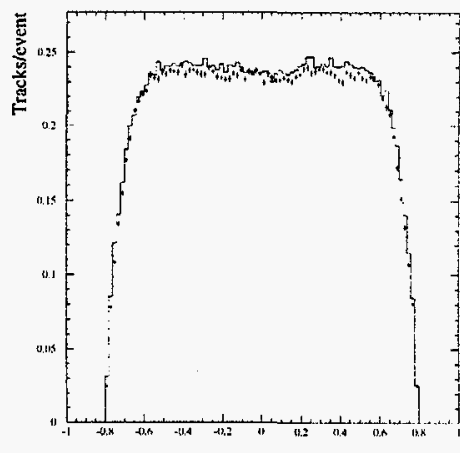

$\cos \theta$

Figure 4.6: Tracking distribution as a function of $\cos \theta$ for data (points) and simulation (histogram), showing a uniform dis. crepancy across the angular region. 


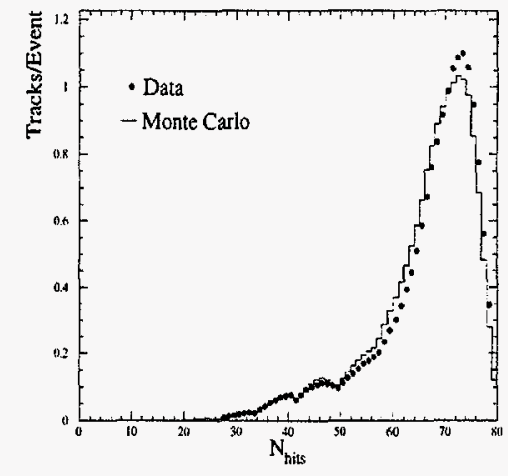

Figure 4.7: Number of hits found on selected tracks.

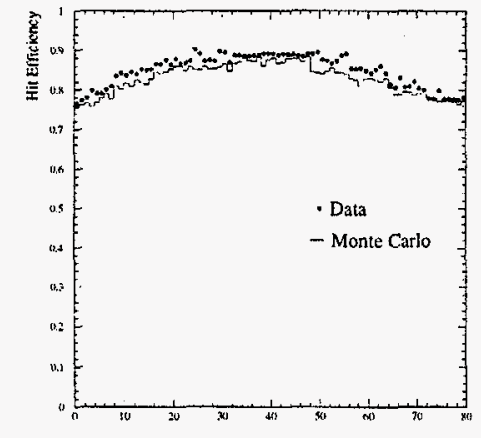

Wire Layer

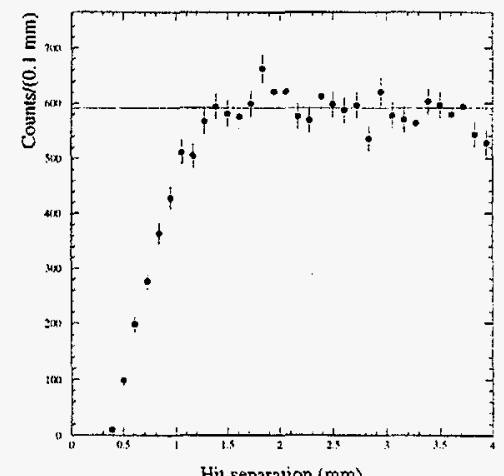

Hit separaiton $(\mathrm{mm})$
Figure 4.8: Hit-finding efficiency as a function of wire layer. The tracks are closer together in the inner layers, and the twohit resolution reduces the hit-finding efficiency there. In addition, electromagnetic backgrounds are more prevalent in the inner layers.

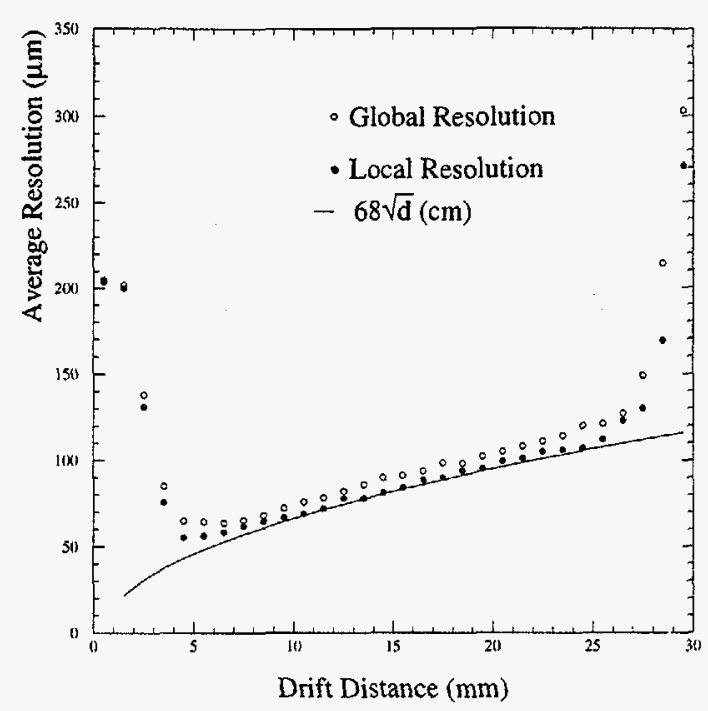

Figure 4.10: Drift distance resolution as a function of drift distance. The resolution worsens at the edges of the cell due to the larger electric fields near the field wires and sense wires, the non-uniformity of the field near the wires, and jonization statistics. The resolution in the central portion of the cell is governed by diffusion.

and more nearly parallel. The two-hit resolution itself may be measured by finding the fraction of the time a hit is found on a track if another track passed close by: Figure 4.9 shows the measured hit-finding probability for closely spaced hits. The distribution of the number of hits on tracks compared with its simulation in Figure 4.7 , supports the claim that the detector's hit recording efficiency and the CDC track finding is simulated well by the Monte Carlo.

\section{Resolution}

Whereas the tracking efficiency is difficult to estimate because the proper number of tracks is unknown, the resolution of the track parameters is straightforward to 
measure. The main ingredients to the position and momentum resolutions of the CDC are the drift distance resolution, the radius of the chamber, the magnetic field strength, and the number of wire layers. The choices of a slow gas and a fast clock on the readout greatly enhance the drift distance resolution, shown in Figure 4.10.

The resolution on the drift distance worsens near the sense wires and the field wires because of the variable strength and direction of the electric field in those regions. The drift velocity increases near the wires, magnifying position errors for finite drift time measurement errors. The resolution in the central region of the cell is dominated by charge diffusion in the drift gas. The slow drift gas also has a small diffusion constant to minimize the drift distance errors in the bulk of the cell.

Two resolutions are shown in Figure 4.10, a global resolution, and a local resolution. The global resolution is found by finding the width of the distributions of track fit residuals as a function of drift distance. The local resolution is obtained by comparing the fit residuals between neighboring hits in a cell. The local resolution is slightly better than the global resolution owing to residual alignment effects arising from the locations of the feedthrough holes in the endplate within their machining tolerances, and tilting of the Lexan blocks within their holes. Alignment corrections cannot be made infinitely precisely, because the equilibrium sag of the wires in the combined electrostatic and gravitational fields within the chamber vary each time the voltage trips off and is restored

The resolution on most hits in the chamber is better than $100 \mu \mathrm{m}$, with an average around $70 \mu \mathrm{m}$. These errors are incorporated into smearing the hit locations in the tracking simulation and are consistent with the observed momentum resolution.

The momentum resolution of the CDC may be estimated from the individual resolutions on the hits, and it may also be measured from $Z^{0} \rightarrow \mu^{+} \mu^{-}$decays. In the absence of hard photon radiation, the momenta of the muons are constrained to be equal and opposite at $45.6 \mathrm{GeV}$. Figure 4.11 shows the distribution of $\mathrm{Q} / \mathrm{P}$ for $Z^{0} \rightarrow \mu^{+} \mu^{-}$decays. This technique probes the momentum resolution the central region of the CDC $(|\cos \theta|<0.75)$, owing to acceptance of the SLD trigger for dimuon events. Tracks which exit the chamber before leaving hits in all layers are expected to have degraded momentum resolution. A fit of two Gaussians to Figure 4.11 yield

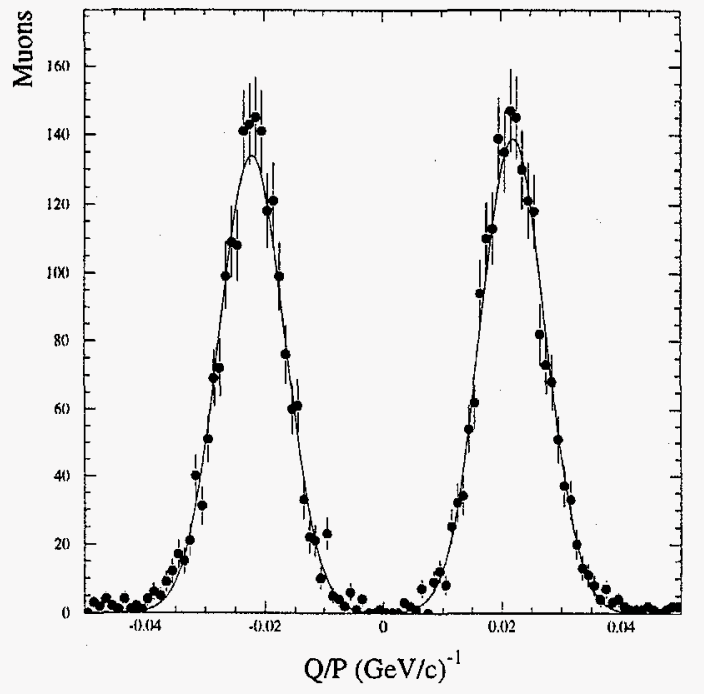

Figure 4.11: Drift chamber curvature measurements for tracks in $Z^{0} \rightarrow \mu^{+} \mu^{-}$events.

the constant term in the error of the curvature measurement. It is also seen that the probability of misassigning the sign of a charged track when it is isolated from other tracks is negligible. On the other hand, tracks may be assigned incorrect hits within dense jets, and charge misassignment becomes possible.

Cosmic ray tracks passing near the center of the CDC provide information about the momentum resolution as a function of momentum by comparing the momenta of the two halves of the track. The derived momentum resolution from these distributions is

$$
d p_{\perp} / p_{\perp}^{2}=\sqrt{0.0050^{2}+\left(0.010 / p_{\perp}\right)^{2}}
$$

where $p_{\perp}$ is the momentum of the particle perpendicular to the beam, measured in $\mathrm{GeV}[108]$.

As an independent verification of the momentum resolution of the chamber and of the absolute scales of the magnetic field within the CDC and its physical dimensions, 


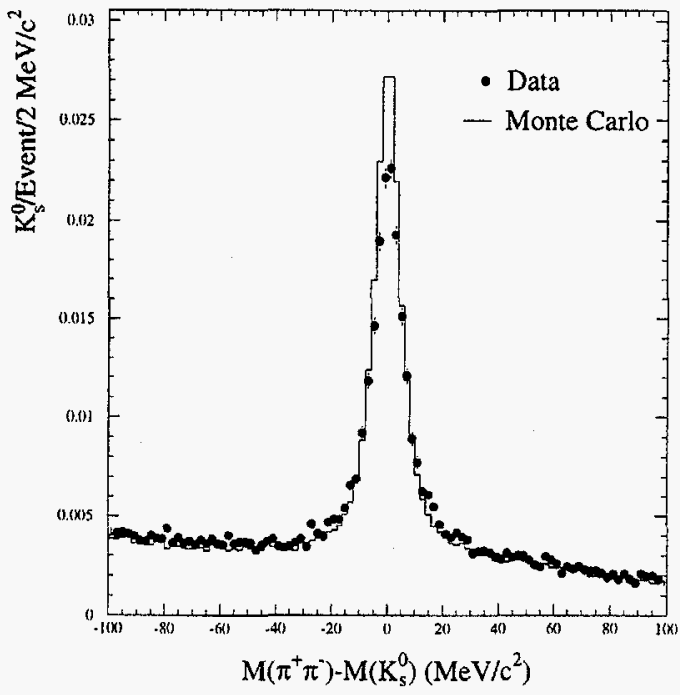

Figure 4.12: Comparison of the $K_{3}^{0}$ mass resolution in data and Monte Carlo. The kaon was required to start at least $10 \mathrm{~cm}$ from the beam axis. The Monte Carlo has a slightly more optimistic momentum resolution than the data.

the invariant mass of $K_{s}^{0} \rightarrow \pi^{+} \pi^{-}$decays may be calculated, as shown in Figure 4.12. The position and width of the peak, as compared with the Monte Carlo, indicate that the simulation accurately reproduces the momentum measurement.

\subsection{Tracking: Vertex Detector}

The SLD vertex detector (VXD) [111][112] greatly improves the measurements of the trajectories of charged particles using silicon CCD pixel detectors.

\subsubsection{Mechanical Design}

The individual $\mathrm{CCD}$ chips are mounted on alumina motherboards, nicknamed
Table 4.1: Vertex detector design parameters.

\begin{tabular}{|lc|}
\hline CCD count & 480 \\
Pixels/CCD & $400 \times 600$ \\
Pixel size & $22 \mu \mathrm{m} \times 22 \mu \mathrm{m}$ \\
Active area per CCD & $8.5 \mathrm{~mm} \times 12.7 \mathrm{~mm}$ \\
Readout time & $160 \mathrm{~ms}(19$ beam crossings $)$ \\
Operating temperature & $170 \mathrm{~K}$ \\
Layer 1 radius & $29.5 \mathrm{~mm}$ \\
Layer 2 radius & $33.5 \mathrm{~mm}$ \\
Layer 3 radius & $37.5 \mathrm{~mm}$ \\
Layer 4 radius & $41.5 \mathrm{~mm}$ \\
Radiation thickness per layer & $L / L_{R}=1.064 \%$ \\
Expected hits/track & 2.3 \\
Two-hit coverage & $|\cos \theta|<0.75$ \\
One-hit coverage & $|\cos \theta|<0.82$ \\
\hline
\end{tabular}

"ladders," with eight chips per board. The CCD's alternately face towards and away from the beampipe along a ladder. The geometrical layout of 60 ladders is shown in Figure 4.14, and the salient parameters of the VXD construction are listed in $\mathrm{Ta}$ bles 4.1. There are gaps in the azimuthal coverage of each layer, but the arrangement of the ladders in the other layers guarantees two-hit coverage everywhere in $\phi$.

The VXD provides $\sim 120$ million pieces of analog information on every event, which takes $160 \mathrm{~ms}$ to read out, allowing 19 beam crossings of background to accumulate. The analog signals are digitized and processed in the VXD data acquisition fastbus modules atop the detector. The first stage is to examine a $3 \times 3$ kernel around each pixel of the detector store the addresses of kernels whose signal passes a threshold criterion $\ddagger$. A Motorola 68020 processor then examines these selected $3 \times 3$ kernels "The chip that examines these $3 \times 3$ kernels, an ASIC called a CAP chip, incorporates an "extended
row filter." This filter forms the differences between neighboring pixels and compares it with the
difference between the neighbors of those pixels. Because the charge accumulates on a CCD row
readout, the task is to look for steps. This filter is effective in removing oscillatory background. 

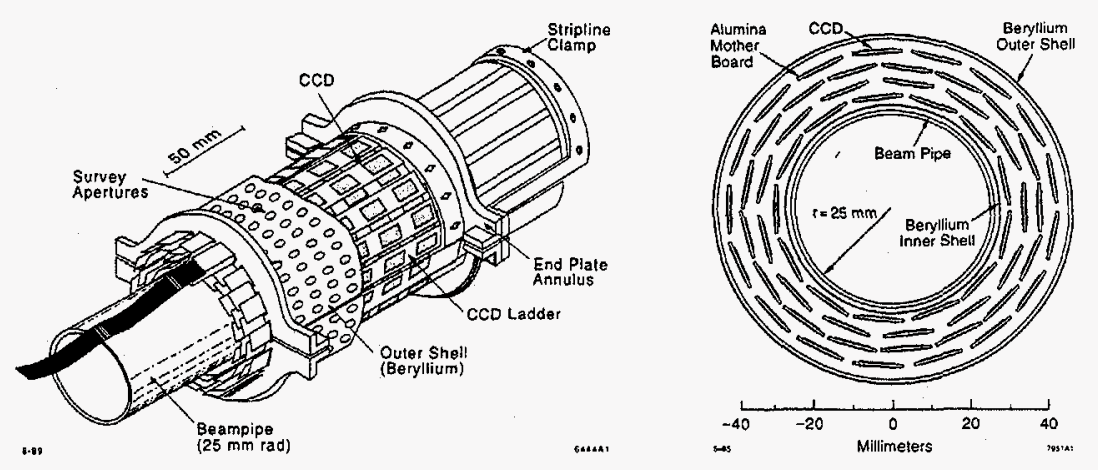

Figure 4.13: Perspective view of the VXD showing support structures and electronic attach ments. The gaps between the CCD's showing on the ladders are covered by CCD's on the opposing faces of the ladders.

\subsubsection{VXD Reconstruction}

The first procedure applied to VXD data is to remove noise hits by identifying clusters which form long streaks in the CCD's. These are created by charged particles produced upstream of the interaction point in $\mathrm{e}-\mathrm{N}$ scattering with the masks and beam walls.

The reconstruction of tracks in the VXD has two stages - finding track links and fitting the combined VXD/CDC track. The first stage has two parts to it: single hit finding and the attaching of a second hit. To find the first VXD hit, each CDC track is extrapolated to the planes of the VXD. A search ellipse is projected onto the CCD plane, with its axes determined by the CDC track error matrix and the angle of incidence. Multiple scattering in the material between the CDC and the VXD layers is taken into account when propagating this error matrix to the CCD plane. If a cluster is found, then the track parameters are adjusted so as to force the track to pass through the cluster. The errors are then recalculated with the cluster"s location providing extra location information. The major source of error at this point is the ability of the track to pivot in angle around the linked cluster. A new error ellipse is projected onto the second VXD layer in which a hit is expected. If a second cluster is found, then the track is fully linked, and a search is made for more clusters because CCD chips overlap partially. Tracks with more than one hit per layer enable an accurate local alignment of the detector.

In case the two-hit finding algorithn fails for a particular track, or in case a second cluster is not expected for a track owing to non-functional detector elements, a second approach is taken. Here, the CDC track parameters are recalculated to force the track through the nominal beam position while minimizing $\chi^{2}$. Then an error ellipse is projected onto a CCD plane where a hit might be expected. If a cluster is found within this ellipse, the track is allowed to pass with only one linked cluster. This technique allows high vertexing efficiency at steeper dip angles and lower momenta. It also improves the uniformity of the azimuthal coverage even with a non-functional ladder.

Adding VXD information to existing CDC tracks is accomplished after the CDC tracks have been fit and the track parameters and full $\mathrm{CDC}$ error matrix found. 

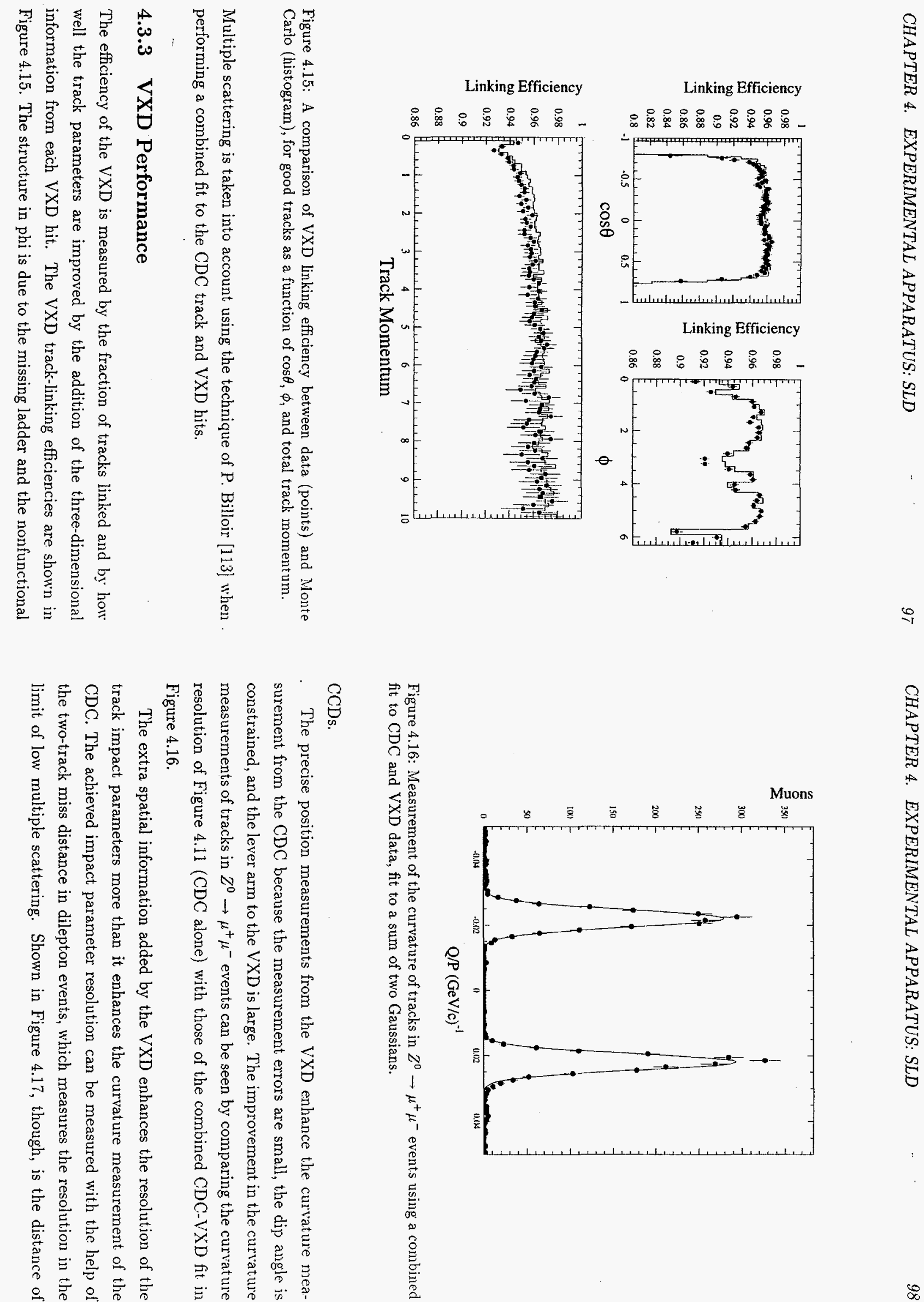

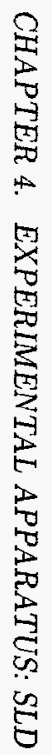




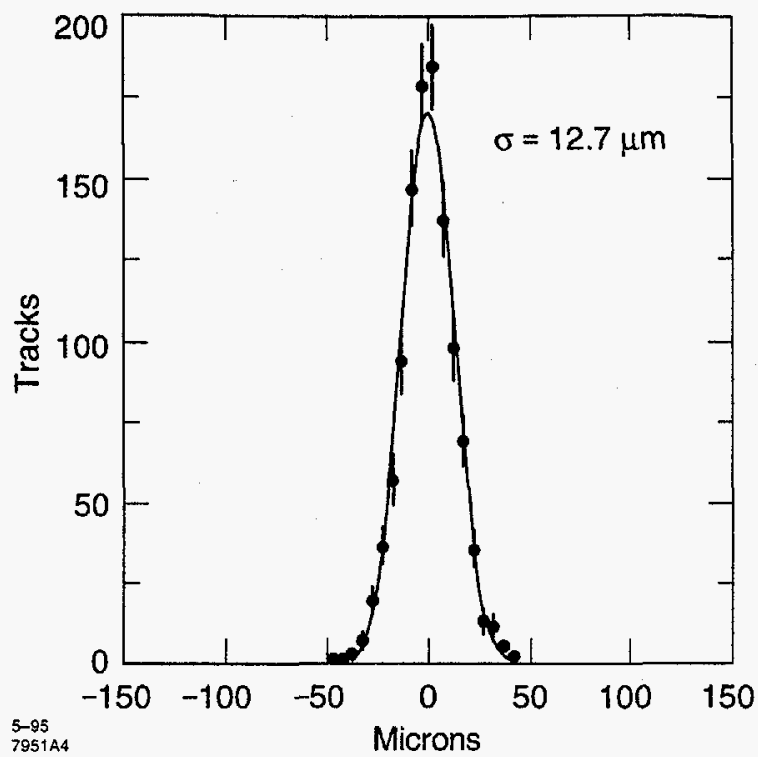

Figure 4.17: Impact parameter of tracks in $Z^{0} \rightarrow \mu^{+} \mu^{-}$events with respect to the average IP position determined from hadronic events, shown fit to a Gaussian of width $12.7 \mu \mathrm{m}$.

closest approach of muons to the fit SLC beam spot, which provides information about the resolution of the fit IP as well. The position of the beam spot is precisely measured by averaging the primary vertices of 30 hadronic $Z^{0}$ decays.

The impact parameter resolution for tracks of arbitrary momenta may be simulated in the Monte Carlo and compared with the data. distributions of the impact parameter, its error, and the impact parameter normalized by its error are shown in Figures 6.7 and 6.8. The agreement between the data and simulation on these variables indicates that the errors are being modeled properly by the Monte Carlo.

The $x-y$ impact parameter error from the track measurement can be roughly parameterized by

$$
\delta b \simeq \sqrt{(11 \mu \mathrm{m})^{2}+70 \mu \mathrm{m} /\left(p|\sin \theta|^{3 / 2}\right)}
$$

where $P$ is the momentum of the track and $\theta$ is its polar angle. The second tem is the contribution from multiple scattering. An additional error of $6 \mu \mathrm{m}$ on the average IP position must be added in quadrature to get the true impact parameter error. This parameterization is only approximate, because hard scattering in the detector material introduces tails in the impact parameter distribution, and because the resolution of the VXD is not uniform in $\phi$ due to a variable lever arm between the hits on the track.

\subsection{Particle ID: Cherenkov Ring Imaging Detector}

The Cherenkov Ring Imaging Detector (CRID) is the particle identification system for the SLD [114]. Although it is not used in the analysis presented in this thesis, it has much to offer that will help future measurements of $A_{b}$ with SLD. The CRID operates by measuring the opening angle of the cone of Cherenkov light emitted as a charged particle passes through a transparent medium in which the index of refraction retards the speed of light below the speed of the particle. This opening angle is given by

$$
\theta_{C}=\cos ^{-1}\left(\frac{1}{\beta \eta}\right),
$$

where $\beta$ is the speed of the particle divided by the vacuum speed of light, and $\eta$ is the index of refraction in the material.

The CRID has a dual radiator structure - a liquid radiator for better resolution of lower energy particles, and a gaseous radiator for distinguishing the identities of higher energy particles. The average number of photons emitted in the Cherenkov cones of a typical particle is about 17 (liquid) and 8 (gas), so the photon detection efficiency must be very high, and the positions of the photons must be recorded very accurately. To achieve this, three-dimensional time-projection chamber (TPC) drift_boxes are installed between the two radiators, separated by transparent quartz windows. The liquid radiator is a thin layer of $\mathrm{C}_{6} \mathrm{~F}_{12}$, and the TPC box is located very close to it. The angular resolution is supplied entirely by "proximity focusing." owing to the thinness of the Cherenkov cone and the closeness of the TPC. The TPC has a second role of detecting photons emitted in the gas radiator, $\mathrm{C}_{5} \mathrm{~F}_{12}$ mixed with $\mathrm{N}_{2}$. Because the path length the track must have in the gas required for it to emit 
sufficient photons is long $(30 \mathrm{~cm})$, the Cherenkov cones cannot be as thin as in the case of the liquid radiator. The Cherenkov light therefore needs to be focused back on the TPC sensitive planes by an array of 400 spherical mirrors mounted on the periphery of the CRID.

The TPCs are filled with a gas containing ethane, argon, and Tetrakis Dimethy] Amino Ethylene (TMAE). Of easily available substances, TMAE has the best quantum efficiency for photoionization. It also has a low energy threshold for photoionization, so the bandwidth of the system is increased. The upper edge of the bandwidth is fixed by the absorption edges of the quartz windows. A different material would have to be chosen for the windows if the sensitive material allowed less bandwidth. TMAE can also be cleaned, and the control on the $\mathrm{O}_{2}$ levels necessary to preserve the electron lifetime is manageable. Ionization drifts towards the sense wires mounted at the ends of the drift boxes. These sense wires are shielded by opaque baffles, owing to the fact that as the ionization avalanche progresses in the high field region near the sense wires, the TMAE fluoresces, and the signal would be picked up on neighboring wires if not shielded. TPC alignment fiducials are supplied by ultraviolet laser light directed along a known trajectory through the detector.

The $K-\pi$ separation can be estimated from the Monte Carlo simulation and checked against data using samples of known composition from $K_{s} \rightarrow \pi^{+} \pi^{-}$decays and $\Lambda \rightarrow p \pi^{-}$decays. In addition, low multiplicity decays of the $\tau$ consist mostly of pions with a well-measured $K$ contribution, and therefore can be used to estimate the $K-\pi$ separation in higher momentum ranges.

An interesting feature of the CRID is that its identification becomes very good when using the Cherenkov threshold region. For the gas, charged kaons do not radiate at all below $\sim 10 \mathrm{GeV}$, while the pion threshold is near $\sim 2.5 \mathrm{GeV}$. The particles can be identified by the lack of a ring. Recently Su Dong has performed an analysis of $A_{6}$ using kaons identified with the CRID [50], estimating an efficiency for tagging kaons in $Z^{0} \rightarrow b \bar{b}$ decays to be $30 \%$ with a background fraction from misidentified pions of $7.2 \%$.

Using the Cherenkov threshold is also important for separating electrons from pions below $4 \mathrm{GeV}$ and could double the electron identification efficiency. In the future,

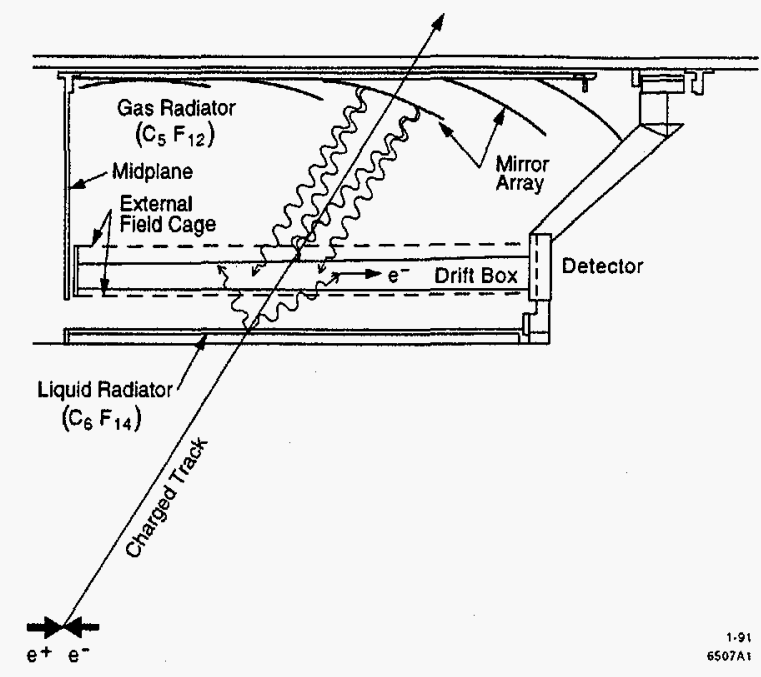

Figure 4.18: Mechanical layout of the barrel CRID

when exclusive reconstruction of $B$ hadrons is attempted, particle identification from the CRID will most likely play a central role.

\subsection{Calorimetry: Liquid Argon and WIC Pads}

The SLD measures particle energy deposition in a Liquid Argon Calorimeter (LAC) [115] and a Warm Iron Calorimeter (WIC) [116]. The majority of particles produced in $Z^{0}$ decay are stopped completely by the LAC, with very little shower leakage. The cylindrical barrel section of the LAC occupies the radial region between $177 \mathrm{~cm}$ and $291 \mathrm{~cm}$, including its cryostat, and it has a full length of $620 \mathrm{~cm}$, again including the cryostat.

The LAC is segmented longitudinally into four layers, EM1, EM2, HAD1, and HAD2. Their thicknesses in radiation lengths and interaction lengths are listed in Table 4.2. The electromagnetic thickness is chosen so that roughly half of the electromagnetic shower energy is deposited in EM1 and half in EM2, with almost no leakage 


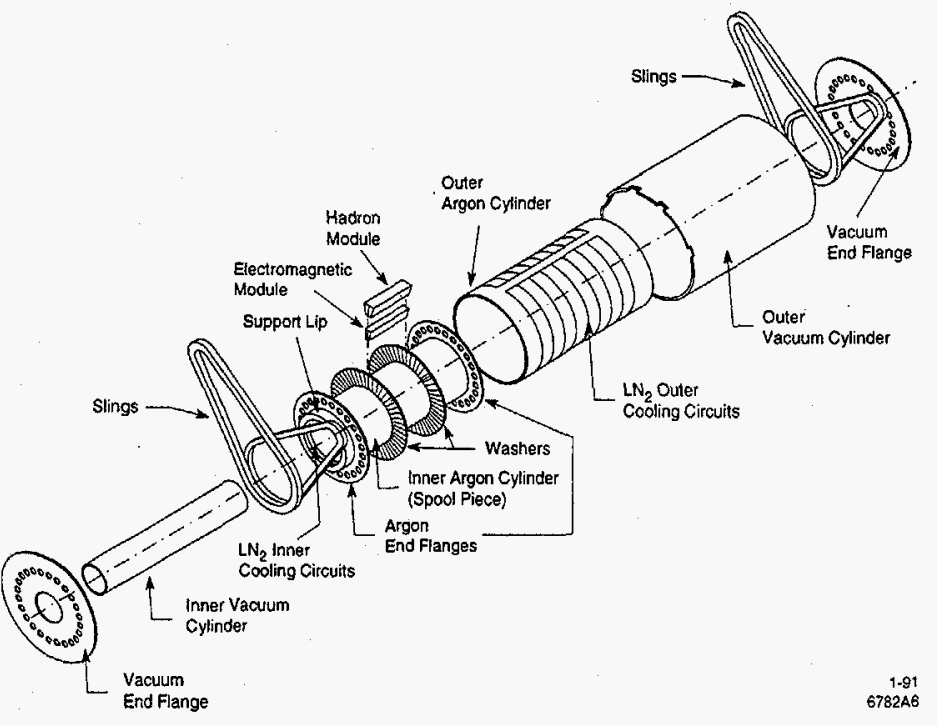

Figure 4.19: Construction of the barrel LAC

into the hadronic layers. The whole LAC is contained within the magnet coil so as not to degrade its energy resolution.

The LAC is finely segmented in polar and azimuthal angles as well. Each angular segment of lead plates is called a "tower," with argon interspersed. The tower dimensions in the electromagnetic layers are $33 \times 36 \mathrm{mrad}$ in $\theta$ and $\phi$ respectively. in the middle of the detector, while the hadronic segmentation is twice as coarse in both directions. The towers are arranged in a projective geometry, allowing for better transverse shower shape measurements.

In addition to the barrel section of the LAC, two endcap LAC sections fill in the coverage at high $\cos \theta$. These are also formed of projective towers of lead and liquid argon, segmented nearly identically to the barrel LAC. It extends the coverage of the LAC out to $|\cos \theta|<0.98$, although there is a region between the barrel and endcap at $|\cos \theta|=0.8470 \mathrm{mrad}$ wide, with degraded energy resolution due to the

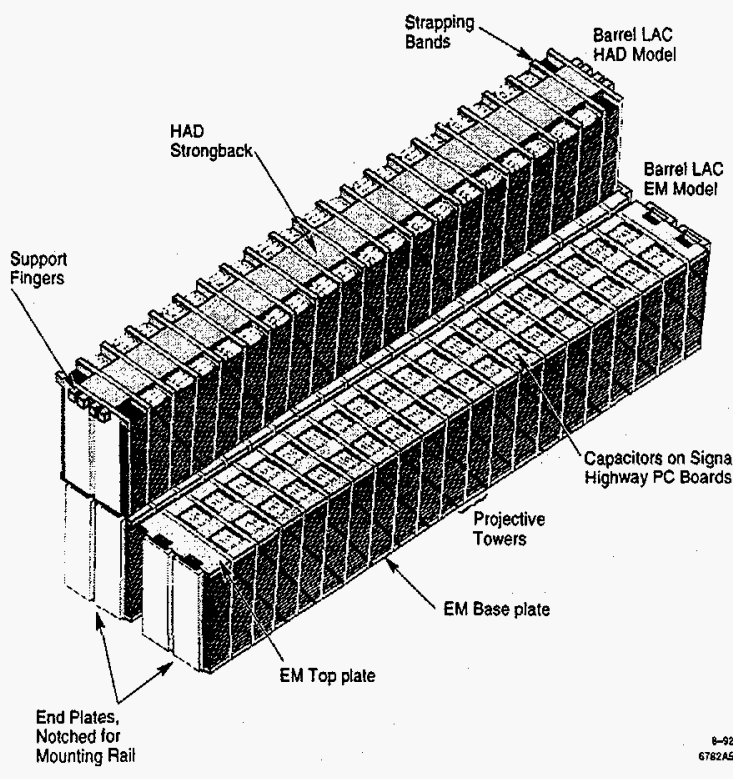

Figure 4.20: A single module of the barrel LAC

Table 4.2: Geometrical properties of the LAC and WIC pads. The thichnesses are reported at normal incidence.

\begin{tabular}{|lccc|}
\hline Layer & Radiation Lengths & Interaction Lengths & Angular Segmentation \\
\hline EM1 & 6.0 & 0.24 & $33 \mathrm{mr}$ \\
EM2 & 15.0 & 0.60 & $33 \mathrm{mr}$ \\
HAD1 & 25 & 1.00 & $66 \mathrm{mr}$ \\
HAD2 & 25 & 1.00 & $66 \mathrm{mr}$ \\
WIC1 & 50 & 2.0 & $66 \mathrm{mr}$ \\
WIC2 & 50 & 2.0 & $66 \mathrm{mr}$ \\
\hline Total & 171 & 6.84 & \\
\hline
\end{tabular}


overlapping cryostats and the loss of hadronic coverage, as can be seen best in the SLD quadrant view (Figure 4.1).

The energy resolution of the LAC has several contributing components. The material between the LAC and the beamline degrades the resolution somewhat in an angledependent way. The intrinsic resolution of the calorimeter depends on the statistics of individual showers, the sampling fraction, and the ability of the calorimeter to respond to the electromagnetic and hadronic components of a hadronic shower with comparable energy scales. The LAC is an uncompensated sampling calorimeter, so its electromagnetic resolution is much better than its hadronic resolution. Material in front of the calorimeter, more prevalent in the endcap regions, affects the electromagnetic resolution more than the hadronic resolution, owing to the fact that it takes more material to start a hadronic shower on average. The average electromagnetic resolution is $\delta E_{e m} / E_{e m} \simeq 15 \% / \sqrt{E_{e m}}$, where $E_{\text {em }}$ is the electromagnetic energy in GeV. The hadronic energy is roughly parameterized by $\delta E_{h a d} / E_{h a d} \simeq 65 \% / \sqrt{E_{h a d}}$. In the endcap LAC, the electromagnetic energy resolution degrades to $\delta E_{e m} / E_{e m} \simeq 25 \% / \sqrt{E_{e m}}$. It is the fine segmentation and good electromagnetic resolution that enables the LAC. to be used, in conjunction with tracking in the CDC, to identify electrons among the decay products of the $Z^{0}$.

Hadronic showers that fluctuate to longer lengths are contained in a secondary calorimetric device, the Warm Iron Calorimeter (WIC). Located outside of the magnet coil, the WIC also functions as a muon identifier, mechanical support, and magnetic flux return for the SLD. It consists of 18 layers of 2 " thick steel plates, with plastic streamer tubes ("Iarocci tubes") [117] in between, accounting for 4 interaction lengths of material. The Iarocci tubes are instrumented with long copper pickup strips for muon tracking and broad pads which record a signal proportional to the ionization count in the neighboring tubes. The segmentation of the pads is in the form of projective towers with the same angular segmentation as the HAD section of the $\mathrm{LAC}$, and divided into two layers longitudinally. Monte Carlo estimates of the energy punchthrough to the WIC are 5\% for typical hadronic showers. The WIC Pads are not used in this analysis.

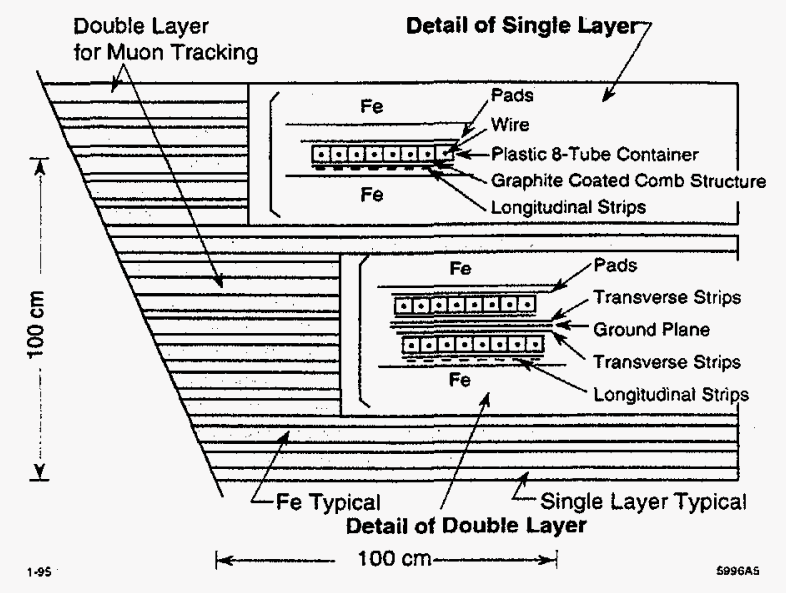

Figure 4.21: Mechanical layout of the Warm Iron Calorimeter.

\subsection{Muon Identification: WIC Strips}

The WIC provides information, though, which aids the study of the forward-backward left-right asymmetries [45][46]. Muons leave a characteristic signal in the WIC, as they are the only charged particles which can penetrate the steel and leave an ionization trail $^{\S}$. The WIC is arranged so that most of the Iarocci tubes, operated in limited streamer mode, are oriented parallel to the beamline. The WIC is organized into eight octants and two endcaps. The support arches are instrumented for coverage in the gap between the barrel octants and the endcaps. Copper strips are mounted along the lengths of the Iarocci tubes, and voltage is applied to the central wire in the tube, while the graphite coating on the tube is maintained at ground.

When a charged track passes through a tube, it ionizes the gas inside. As the oppositely charged ions travel to the field wire and to the graphite coating, an image charge builds up on the copper strip outside, producing a signal, which may be detected with sensitive amplifiers on the ends of the strips. The only information recorded by the WIC strips, though, is whether a strip was struck, not the magnitude

\footnotetext{
${ }^{\S}$ Except for a small amount of pion punchthrough.
} 
of the charge deposited. Because the ionization signal saturates the tube in limited streamer mode, no more information need be recorded. The tube size is $1 \mathrm{~cm} \times 1 \mathrm{~cm}$, so the fine segmentation of the system allows muon tracking with a positional resolution of $1.0 \mathrm{~cm} /$ hit.

\section{Chapter 5}

\section{The SLD Monte Carlo}

\subsection{Introduction}

Many analyses at the $Z^{0}$ rely on Monte Carlo models of both the detector and the underlying physical processes involved in the hard scattering under study. These models are indispensable for determining precisely measurement biases induced by real detectors with finite acceptances, efficiencies, and resolutions. If an analysis uses several subsystems of the detector, the modeling of the interplay between the efficiencies and acceptances of the different subsystems can become critical.

Analyses also need to correct for physical processes that are not directly under study. In particular, the analysis of measuring $A_{b}$ with momentum-weighted track charge is sensitive to the details of $B$ hadron fragmentation and decay, the properties of which have been measured at other experiments more accurately than they can be determined by SLD alone. These properties are incorporated into the physical model used, and uncertainties in them propagate into systematic errors in the final result.

The SLD Monte Carlo consists of two separate functional units: an event generator, which models the underlying physics of $Z^{\circ}$ decay and the physics of its unstable products, and a detector simulation model. 


\subsection{The Event Generator}

The event generator chosen for this analysis is the JETSET 7.4 model [30], incorporating LUND string fragmentation. The electroweak processes have been checked against the forms of Chapter 1 , including the effects of $\gamma-Z^{0}$ interference. The SLD Monte Carlo generates half of its events with an electron beam polarization of $+100 \%$, and half with $-100 \%$, with the positron beam unpolarized in both cases. In order to simulate properly the left-right asymmetry, a fraction of the right-handed events must be eliminated from the analysis, given by

$$
f_{R, t o s s}=\frac{2\left|P_{e}\right| A_{e}}{1+\left|P_{e}\right| A_{e}}
$$

where in this case $\left|P_{e}\right|=1.0$. Once the requisite balance of left-handed and righthanded events is achieved, the polarization in the Monte Carlo may be diluted by re-signing the polarization of a fraction of events.

$$
f_{\text {re-sign }}=\frac{\left|P_{e}\right|-P_{\text {des }}}{2\left|P_{e}\right|}
$$

where again $\left|P_{\mathrm{e}}\right|$ is the generated polarization of $100 \%$, and $P_{\text {des }}$ is the desired polarization.

JETSET implicitly includes initial- and final-state photon radiation, and uses a parton shower model [31] for final-state gluon radiation. Jetset also offers matrix element calculations of gluon emission to first- and second-order in $\alpha_{s}$, and then uses the parton shower model for the higher-order effects. Rather than used a mixed approach, the SLD event generation uses a pure parton shower. This also has the advantage of the fact that JETSET retains the direction of the quark as it is emitted from the $Z^{0}$ decay, which may be compared against the quark direction after the parton shower, in order to calculate the QCD correction to asymmetry measurements.

The fragmentation functions for light-flavored hadrons* is governed by the Lund symmetric function [30] of Equation 1.42, while that for heavy-flavored hadrons is the Peterson formula [118], given in Equation 1.44. These functions can be seen in Figure 1.2.

\footnotetext{
*hadrons containing only $u, d$, and $s$ quarks
}

Table 5.1: Mixing parameters of the SLD Monte Carlo.

\begin{tabular}{|lccc|}
\hline Species & Fraction & $x$ & $\chi$ \\
\hline$B^{0}$ & $40.6 \%$ & 0.75 & 0.180 \\
$B_{s}$ & $11.5 \%$ & 10.0 & 0.495 \\
Average B hadron & $100 \%$ & - & 0.130 \\
\hline Charmed Hadrons & & 0 & 0 \\
\hline
\end{tabular}

\subsection{Mixing and Decay of $B$ Mesons}

The SLD Monte Carlo models the mixing of heavy mesons separately for each species. The decay distribution for a particle which starts as a $B^{0}$ at time $t=0$ is

$$
\begin{aligned}
& P_{B^{0}}=\frac{1}{2} e^{-\Gamma t}[1+\cos \Delta m t], \quad \text { and } \\
& P_{\bar{B}^{0}}=\frac{1}{2} e^{-\Gamma t}[1-\cos \Delta m t],
\end{aligned}
$$

where $\Gamma$ is the reciprocal of the $B^{0}$ lifetime, and $\Delta m$ is the splitting between the mass eigenstates of the $B^{0}$ system. The time-integrated mixing parameter $\chi^{\prime} B^{0}$ can be expressed in terms of these quantities:

$$
\chi_{B^{0}}=\frac{N_{\bar{B}^{0}}}{N_{\bar{B}^{0}}+N_{B^{0}}}=\frac{1}{2} \frac{x^{2}}{1+x^{2}},
$$

where

$$
x=\frac{\Delta m}{\Gamma}
$$

is the parameter most often used in measurements of the time dependence of mixing. The values used in the SLD Monte Carlo are species dependent,and are listed in Table 5.1. The species-averaged mixing parameter for $B$ hadrons is denoted $\chi_{B}$.

JETSET 7.4 is used in the SLD generator to decay all unstable ${ }^{\dagger}$ particles except the $B^{0}$, the $B^{-}$, and the $B_{s}$, and their antiparticles. This strategy was chosen because the decay particle spectra of JETSET decays of those mesons disagree with available data from CLEO and ARGUS. The previous SLD event generator was based on

\footnotetext{
${ }^{\dagger}$ The $K_{s}^{0}$ and the $\Lambda$ are also not decayed. This point will be brought up in Section 5.5.1.
} 
Table 5.2: SLD semileptonic $B$ meson decay branching modes, after Reference [120]. The individual $D^{* *}$ fractions are broken down in Table 5.3. There is no simulation of $B \rightarrow$ $D^{* *} \tau \bar{\nu}$, as there is for the other leptons. The quantity for that column represents the non-resonant $B \rightarrow D^{(*)} \pi \tau \bar{\nu}_{\tau}$ modes present in the simulation.

\begin{tabular}{|lcc|}
\hline Decay Mode & Branching Fraction $(e, \mu)$ & Branching Fraction $(\tau)$ \\
\hline$B \rightarrow D l \nu$ & $2.554 \%$ & $0.4 \%$ \\
$B \rightarrow D^{*} l \nu$ & 5.874 & 1.1 \\
$B \rightarrow D^{* *} l \nu$ & 2.532 & $1.0 \%$ \\
\hline Total & $10.96 \%$ & $2.5 \%$ \\
\hline
\end{tabular}

JETSET 6.3 , which had even worse disagreements with the available data and thus had to be extensively tuned [119]. Rather than re-tune every upgraded version, it is simpler and more correct to divert the decays of $B$ mesons to an alternative package. The package chosen was the CLEO decay' simulation, described below.

\subsubsection{Semileptonic Decays}

The CLEO decay package simulates decays of $B \rightarrow l v X$ using the model of Isgur, Scora, Grinstein, and Wise (ISGW) [121]. The original paper by these authors only treated decays $B \rightarrow D l \nu$ and $B \rightarrow D^{*} l \nu$, but since then a set of $D^{* *}$ states has been identified [122]. Available spectra from CLEO, shown in Figure 5.1, are precise enough to demonstrate that the ISGW model will not fit unless a fraction of $B \rightarrow D^{* *} l \nu$ decays is incorporated. Previous Monte Carlo models invoked nonresonant $B \rightarrow D \pi l \nu$ decays, but it has been shown that only resonant decays are necessary at the current precision to match the available inclusive lepton spectra [120]. SLD's branching fractions for the semileptonic decay modes are shown in Table 5.2. Furthermore, the calculations of ISGW assume that the leptons are massless. A modification of the CLEO code for the SLD environment was made in order to keep the final-state particles on their respective mass shells by re-scaling the momenta of all decay products, making a negligible contribution except in the case of $B \rightarrow \tau \nu X$.

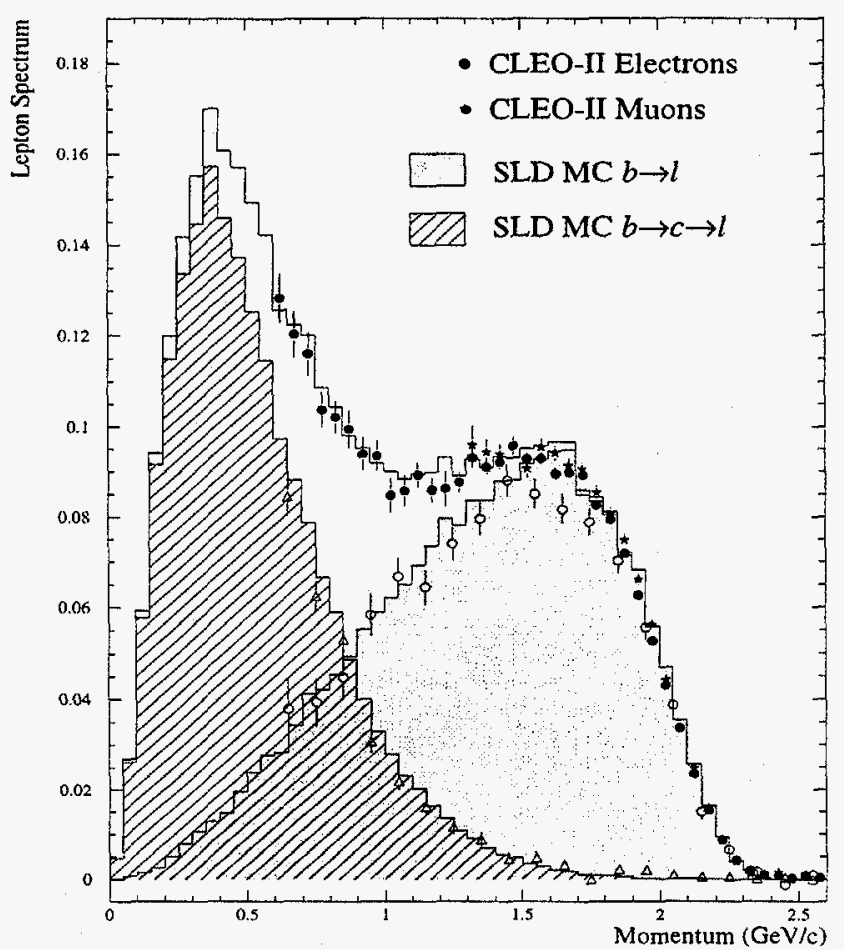

Figure 5.1: A comparison of the semileptonic decay spectra of a Monte Carlo sample composed of $50 \% B^{0}$ decays and $50 \% B^{ \pm}$decays, compared with data taken at the $\Upsilon(4 S)$ by CLEO [120]. Open circles represent inferences of the prompt $B \rightarrow l$ signal from double tags. The momentum spectrum in the Monte Carlo has been smeared to account for the additional boost the $B$ mesons receive at the $\Upsilon(4 S)$. The Monte Carlo contributions from prompt $B \rightarrow l$ and cascade $B \rightarrow D \rightarrow l$ are shown shaded and hatched, respectively. The CLEO data have been corrected for bremsstrahlung both at the lepton production vertex and in the detector material, and also have had $B \rightarrow J / \psi X, B \rightarrow \tau \nu X$, and $B \rightarrow D_{s} X$ decays subtracted. 
Table 5.3: Relative fractions of different $D^{* *}$ states generated in $B$ decay in the SLD Monte Carlo. Fractions originate from the CLEO Monte Carlo [123].

\begin{tabular}{|cc|}
\hline$D^{* *}$ State & Relative Fraction \\
\hline $3 P^{0}$ & $12.3 \%$ \\
$3 P^{1}$ & $24.7 \%$ \\
$1 P^{1}$ & $45.7 \%$ \\
$3 P^{2}$ & $17.3 \%$ \\
\hline
\end{tabular}

\subsubsection{Hadronic Decays}

The hadronic decays of the $B$ mesons are considerably less well constrained. Fortunately, a large fraction ( $45 \%$ ) of hadronic $B$ decays can be reliably tabulated in known exclusive modes [123]. The charmonium states in particular leave distinctive leptonic signals in the detector, and knowledge of the branching fractions of charmonium into leptons allows inference of the charmonium content of $B$ meson decay. But a large portion of hadronic decays must be modeled in a more inclusive manner, and the parameters of the model tuned to bring the inclusive spectra in agreement with available data.

The CLEO model handles hadronic decays by using a $V-A$ matrix element for the $W$ emission and decay, and then, after choosing a final-state multiplicity, fragments the two quarks from the $W$ decay. The available parameters that one may tune are the pseudoscalar fractions for the possible final-state mesons and the relative popping fractions of the quark species in the fragmentation process. These parameters are given in Table 5.4.

While neither CLEO nor ARGUS observes decays of the $B_{s}$ for kinematic reasons, Monte Carlo models may extrapolate from tuned models of $B^{0}$ and $B^{-}$mesons to the $B_{s}$ by replacing the light spectator with a strange quark. The $\Lambda_{b}$, on the other hand, is allowed to be decayed by JETSET.

Comparison of the CLEO decay model with data from CLEO [128] and ARGUS [124][125] are shown below. The total inclusive multiplicity observed by ARGUS is
Table 5.4: CLEO decay model hadronic tuning parameters.

\begin{tabular}{|lc|}
\hline Parameter & Value \\
\hline$s$ popping fraction & $20.0 \%$ \\
Light pseudoscalar fraction & $40.0 \%$ \\
Strange pseudoscalar fraction & $50.0 \%$ \\
Charm pseudoscalar fraction & $15.0 \%$ \\
\hline
\end{tabular}

Table 5.5: Comparison of inclusive multiplicities of $\pi^{ \pm}, K^{ \pm}$, and $P / \bar{P}$ between the CLEO/SLD decay model and ARGUS measurements [124],[125].

\begin{tabular}{|ccc|}
\hline Quantity & Argus $\Upsilon(4 \mathrm{~S})$ Data & CLEO/SLD Model \\
\hline$\left\langle n_{c h}\right\rangle$ & $10.81 \pm 0.05 \pm 0.23$ & 11.04 \\
Total $\pi^{ \pm}$ & $8.21 \pm 0.05 \pm 0.16$ & 8.40 \\
$\pi^{ \pm}$, no $K_{s}^{0}$ or $\Lambda$ decay & $7.17 \pm 0.05 \pm 0.14$ & 7.46 \\
\hline$K^{ \pm}$ & $1.55 \pm 0.03 \pm 0.05$ & 1.49 \\
Total $P / \bar{P}$ & $0.160 \pm 0.010 \pm 0.010$ & 0.13 \\
$P / \bar{P}$, no $\Lambda$ decay & $0.110 \pm 0.010 \pm 0.007$ & 0.11 \\
\hline
\end{tabular}

Table 5.6: Comparison of branching fractions of $B$ mesons at the $\Upsilon(4 \mathrm{~S})$ to the different $D$ mesons. Data are a CLEO/ARGUS average in August, 1994 [127].

\begin{tabular}{|ccc|}
\hline Quantity & CLEO $\Upsilon(4 S)$ Data & CLEO/SLD Model \\
\hline $\mathcal{B}\left(B \rightarrow D^{0} X\right)$ & $(62.1 \pm 2.6) \%$ & $64.8 \%$ \\
$\mathcal{B}\left(B \rightarrow D^{+} X\right)$ & $(23.9 \pm 3.7) \%$ & $26.6 \%$ \\
$\mathcal{B}\left(B \rightarrow D_{s}^{+} X\right)$ & $(10.0 \pm 2.5) \%$ & $10.7 \%$ \\
\hline
\end{tabular}


$10.81 \pm 0.05$ (stat.) \pm 0.23 (syst.), as compared with 11.04 in the SLD tuned Monte Carlo. Most distributions agree fairly closely, except the very highest momentum tail of the inclusive pion spectrum.

The comparison of the $D$ momentum spectrum from $B$ decay, seen in Figures 5.3 and 5.4, shows the advantages of using the CLEO model. The D vector fraction was increased for the SLD version of the Monte Carlo because the $D^{0}$ and $D^{+}$inclusive branching fractions disagreed with CLEO data. In order to raise the $D^{0}$ inclusive fraction, the vector fraction of charm mesons was increased, because of the preference for charmed vector mesons to decay to the $D^{0}$ state, owing to a small difference between the masses of the final states and the small phase space for the $D^{*}$ decay. The momentum spectra of $D$ mesons from $B$ decay in the CLEO model is in agreement with the available data.

The corresponding comparison for JETSET 7.4 shows a $D$ spectrum that is too hard, which would bias the estimated analyzing power of the momentum-weighted track charge technique.

\subsection{Decays of Charmed Mesons}

In order to model correctly the observed final-state particles from $B$ hadron decay, it is important to model correctly the decays of heavy daughter hadrons, in particular the charmed hadrons. The SLD Monte Carlo uses the JETSET decay model for charmed hadrons [31], but the tables of branching fractions have been adjusted by Su Dong [129] to agree better with CLEO, ARGUS, and MARK III data. These modified tables of the decay channels are supplied in Appendix $\mathrm{E}$ for completeness.

\subsection{Detector Simulation}

The Monte Carlo needs to simulate the efficiency, acceptance, and resolution effects for observables of interest to physics analyses. Equally important, though, is the need to incorporate in the models the efficiency of the particle reconstruction algorithms applied to the data. To accomplish these dual purposes, the output of the plyysics
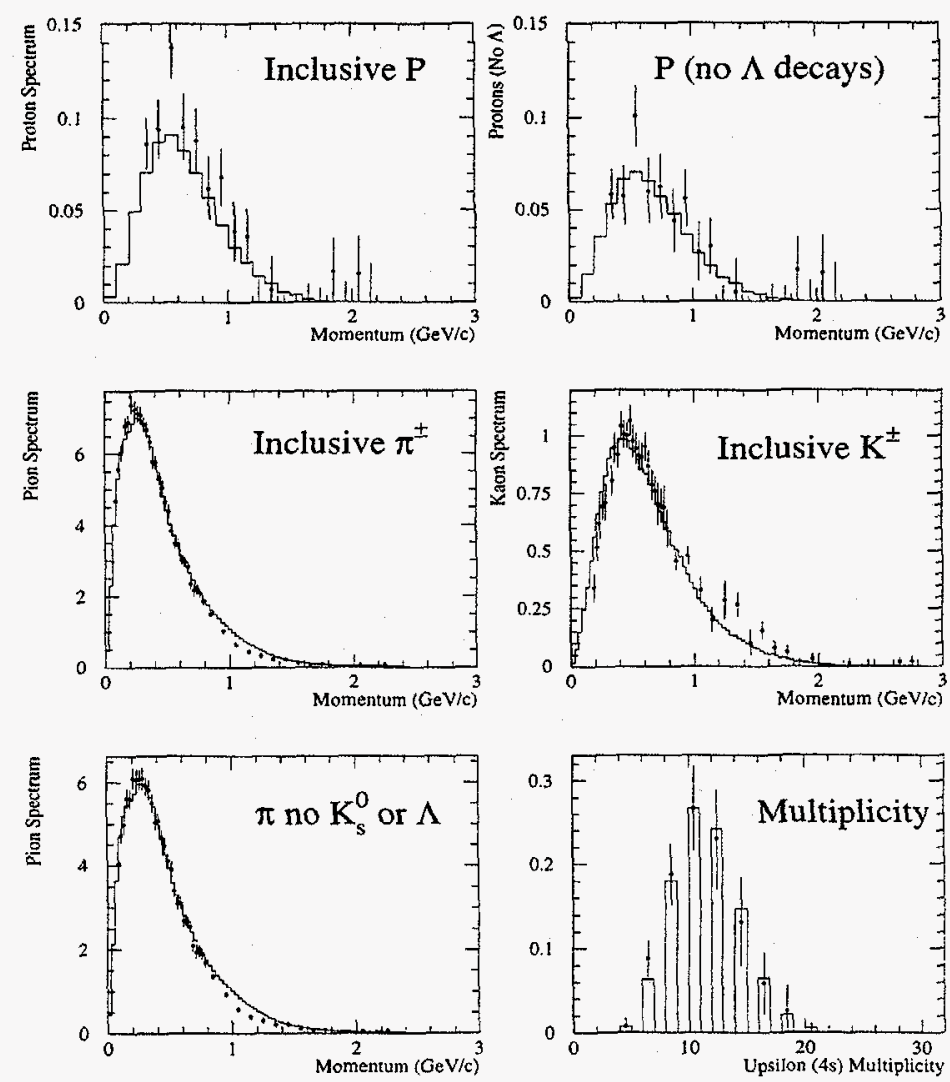

Figure 5.2: A comparison of the SLD-tuned CLEO $B$ meson decay model (histogram) with inclusive particle spectra from Argus [124] (points). The Monte Carlo sample is a $50 \% B^{0}$ and $50 \% B^{ \pm}$sample, with the decay products boosted into the lab frame assuming the $B$ meson was boosted from the $\Upsilon(4 S)$ decay. 

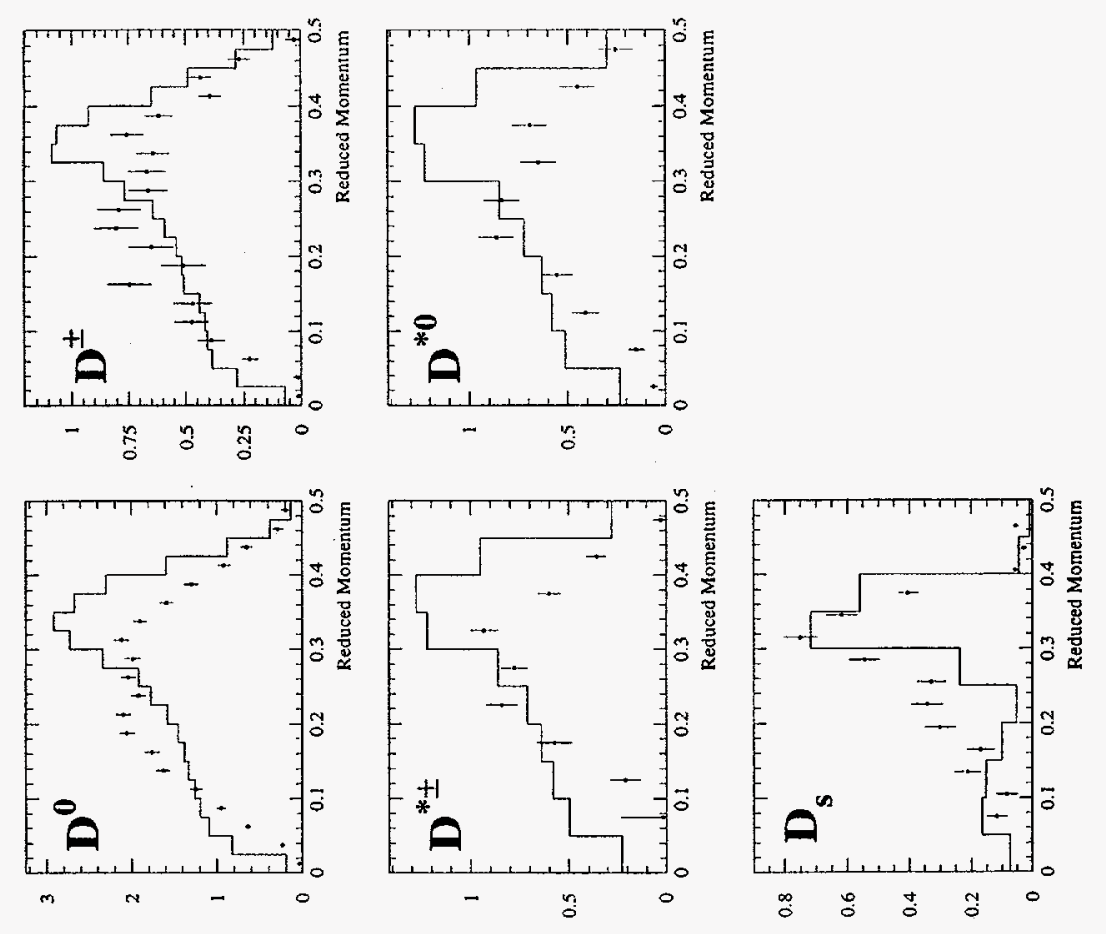

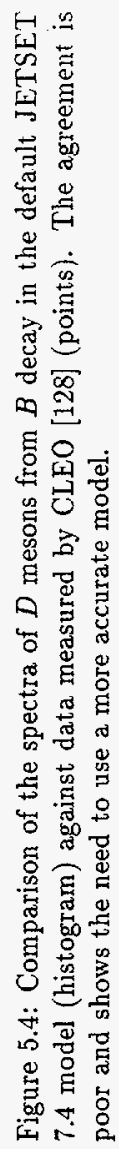

$\triangleq$


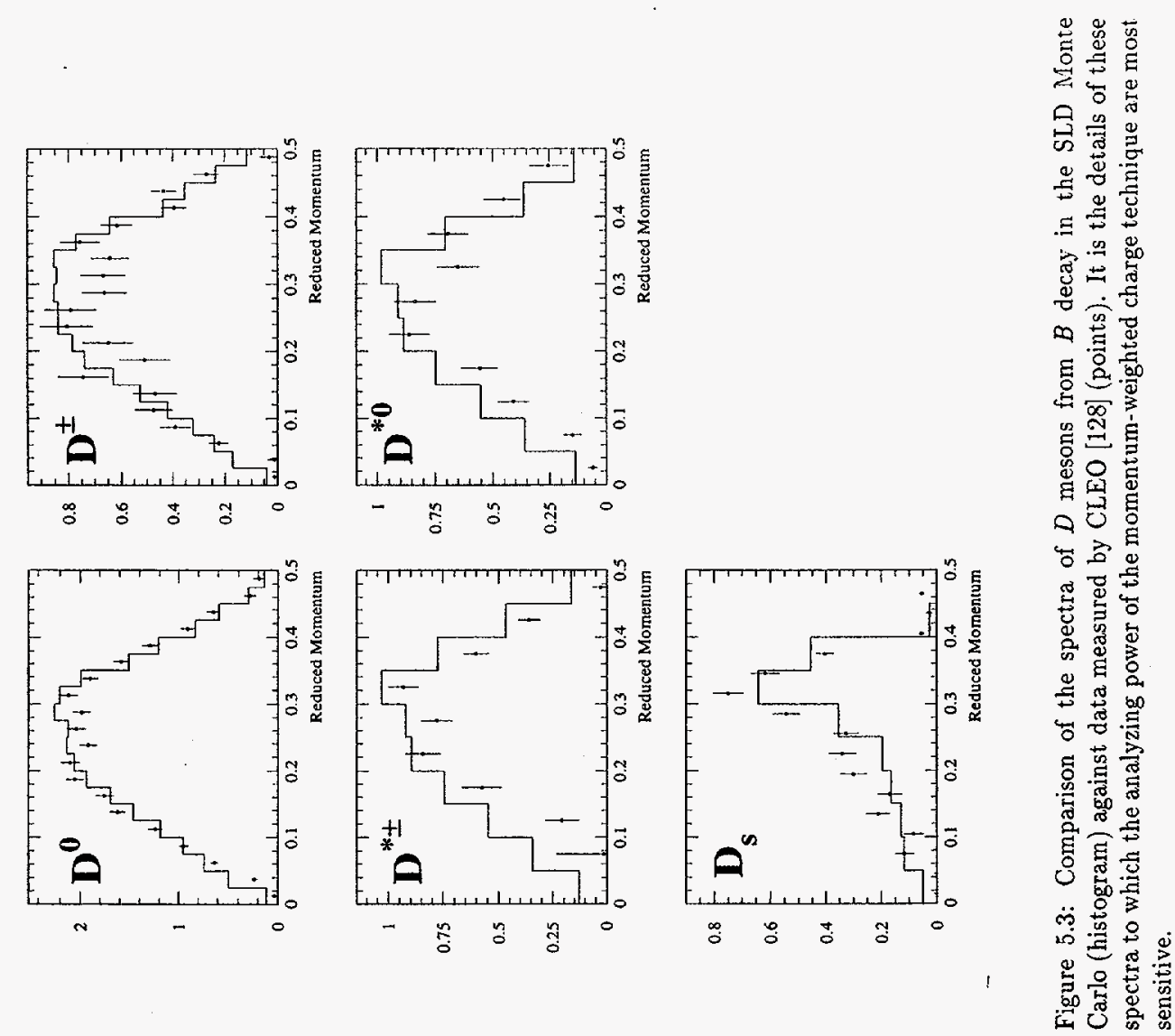
simulation process must be in the same format as the data that is written to tape by the detector and data acquisition, so that the same reconstruction programs may be applied to simulated data as are applied to the actual data.

The input to the simulation is the set of final-state particles created by the event generator. The types, positions, momenta, and the histories of the particles as they traverse the detector must be preserved through the simulation, so that studies of efficiency may be performed. It is also often necessary to isolate model dependence arising from the generator from systematic uncertainties arising from the detector response, so the underlying event is often of great use, even after full detector simulation.

\subsubsection{Detector/Particle Interactions}

The resolution and efficiency of the detector subsystems depend strongly on the amount and location of material particles must pass through. Multiple scattering degrades position and momentum resolution, and electromagnetic or hadronic showers initiated in the detector material before or within the tracking volume introduce extraneous particles whose effect on the analysis must be understood.

Nearly all particles from the interaction region stop in the calorimeter, but not after showers of secondary particles are created. Some of these particles emerge from the calorimeter traveling back into the tracking volume of the detector.

These interactions must be simulated by the Monte Carlo to evaluate detector performance and to adjust the analysis for possible biases. In order for measurements from SLD to be directly comparable with those from other experiments, these interactions must be treated in a consistent manner, incorporating the best knowledge of interactions of high-energy particles and stationary material. To meet this need, the CERN Application Software Group has written a particle-detector simulation package called GEANT [130]; SLD uses Version 3.21.

GEANT starts with a list of particles from the $Z^{0}$ decay generator, a detailed description of the detector material, and a magnetic field map. It then traces the particles' trajectories until they reach boundaries of detector material, at which point GEANT calculates the interaction probability per unit of path length and randomly chooses whether or not to simulate an interaction of the particle with the detector. Geant includes the software routines from EGS4 [98] for simulating electromagnetic interactions and from GEISHA [131] for simulating hadronic interactions.

The final-state particles from the generator also include long-lived particles such as the $K_{s}^{0}$ and the $\Lambda^{0}$. These particles must not be decayed by JETSET as particles with shorter lifetimes are, because they often decay in the detector volume. They may therefore interact with the detector material before decaying.

Interactions with the calorimeter are particularly important, as both the physical layout of the material within the calorimeter and the details of the modeling of interactions between high-energy particles and the material affect the calorimeter's energy and spatial resolution.

One of GEANT's options is to simulate in full detail the showers of particles in the calorimeter by calling the GEISHA and EGS packages. Due to the high multiplicity of $e^{+} e^{-}$pairs in high-energy electromagnetic showers, this process consumes large amounts of computer time. This very multiplicity, however, reduces the relative statistical fluctuations in energy deposition. In addition, the transverse width of electromagnetic showers is small. These properties enable successful parameterizations which deposit energy in the sensitive elements according to carefully selected probability functions [133]. These functions describe the longitudinal development of the showers and also their transverse extent as a function of the shower depth. The simulation used by SLD is based on the GFLASH shower parameterization [132] developed by the $\mathrm{H} 1$ collaboration.

\subsubsection{Digitization}

The next step after GEANT creates its list of new particles formed by detector interactions and modifies the flight paths of old ones due to multiple scattering is to simulate the detector response to all of the particles. This stage incorporates information about which portions of the detector consist of sensitive material.

The VXD digitization simulates the charge deposited in the depletion layer of the silicon, as well as the efficiencies of the different CCD chips for recovering this charge. It also simulates random misalignments of the $\mathrm{CCD}$ chips by randomly fluctuating 
the CCD locations on each event based on their alignment errors ${ }^{\ddagger}$.

Digitization for the CDC involves interpolating each charged particle's track to the charge collection plane for each wire that is to receive a signal from that track. The purpose is to find the closest distance from the track to the wire along the electric field lines, in order to calculate the time of the leading edge of the CDC pulse. The total ionization is calculated as a function of the particle's type, its momentum, and its total path length through the active region of the cell. The simulated charge is then distributed between the north and south readouts of the wire. Some fraction of the amplifiers and digitizers do not function, and the set of these, as determined from the data, are listed in blocks of time. If a set of electronics (usually confined to a motherboard) is non-functional in the data for a particular time, the Monte Carlo simulation of its signal will also not be present. The time of the event is taken from the background overlay event, to be discussed in Section 5.5.3. Also taken from the background overlay event is information about the state of the high voltage in each of the 10 superlayers of the CDC. If the voltage is not at least $95 \%$ of its nominal value, the layer is considered to be tripped off, and the Monte Carlo digitization does not deposit hits in the cells of that layer.

The digitization also takes into account known physical effects, such as the two-hit resolution of Figure 4.9. Additional hits are simulated at the digitization stage called "shadow hits." These are found in the data as hits on wires immediately after hits on real tracks, and typically have $40 \%$ of the charge or less. They are found more on tracks which have large charge deposition lengths and low momentum. On average, roughly $10 \%$ of real track hits are followed by shadow hits. It is hypothesized that they come from oscillations in the electronics and/or crosstalk between neighboring wires and/or the different arrival times at the sense wires of "clumps" of ionization. Some of them may be due to delta rays from the tracks, but these would produce an equal distribution of shadow hits on either side of the tracks. Only a small fraction of shadow hits are observed to precede the main track hit.

\footnotetext{
${ }^{\ddagger}$ The real misalignments are constant, but unknown, so a random fuctuation is not a strictly correct model. Nonetheless, a constant misalignment of the CCD's in the Monte Carlo introduces biases just as bad those in the data with no information about whether they would augment or cancel the data biases.
}

The LAC is digitized in combination with the showering process of GEANT. The parameterized showers simulate energy depositions in the towers. The hadronic tracks' path lengths are found in the towers of the LAC and their energy is deposited using the minimum-ionizing scale as determined from cosmic-ray muon events. Also simulated are dead towers and towers with low energy response. WIC pads digitization is grouped with the LAC digitization owing to its similar tower structure.

\subsubsection{Background Overlay}

Accelerator-related backgrounds are difficult to simulate with Monte Carlo models they are highly variable in time and have characteristics very different from particles from $Z^{0}$ decay. Muons generated far upstream traverse the calorimeter lengthwise, either passing through only lead tiles, or passing only through sensitive argon. Lowenergy electrons looping in the magnetic field raise the occupancy of the inner tracking layers. Sometimes a spray of background particles strikes the electronics on the CDC endplate, saturating some of the amplifiers and causing all of the amplifiers in the immediate vicinity to oscillate. These backgrounds introduce hit-finding inefficiencies in the tracking chambers and add background energy to the calorimeter.

The best way to simulate accelerator-related backgrounds is to measure them from the data. For each $Z^{0}$ event identified in the data sample, a random trigger (see Section 6.2.1) taken at a nearby time is also culled from the raw tapes. The signals from the random trigger are then merged with the digitized signals from the Monte Carlo simulation. For the calorimeter, the tower energies are simply added, but for the CDC, the two-hit resolution is applied. If a background hit comes immediately before a Monte Carlo simulated hit on a track, the later hit will be lost in the simulation. 


\subsubsection{Technique Overview}

To extract the value of $A_{b}$ using the left-right forward-backward asymmetry, the values of two observables must be measured for a sample of $Z^{0} \rightarrow b \bar{b}$ events. The first is the $b$ quark direction relative to the incident electron direction, expressed as

$$
\cos \theta=\vec{p}_{b} \cdot \vec{p}_{e^{-}} / p_{b} p_{e^{-}}
$$

\section{$A_{b}$ with Momentum-Weighted Charge: Binned Asymmetry}

\subsection{Introduction}

This chapter and the next present experimental determinations of $A_{b}$ using the polarized forward-backward asymmetry* of $b$ quarks from $Z^{0}$ decay. The sample of $Z^{0} \rightarrow b \bar{b}$ events was isolated using a lifetime tag [134], and discrimination between the $b$ and $\bar{b}$ directions of the decay axis was provided by the event momentum-weighted charge. The SLD Monte Carlo is used in this chapter to estimate the tag composition, the probability the decay axis is correctly signed, and the magnitude of radiative effects.

The remainder of this section will present a brief outline of the technique and justify its use. Following that is a detailed presentation of the measurement. its systematic errors, and cross-checks that have been investigated. Chapter 7 presents a second version of the analysis which reduces the systematic error by deriving its calibration from experimental data.

\footnotetext{
"Also called the "left-right forward-backward asymmetry."
}

where $\vec{p}_{b}$ and $\vec{p}_{e^{-}}$are the $b$ quark and electron momenta, respectively. In general, the $\bar{b}$ quark will travel in the direction opposite to the $b$ quark. The second is the electron beam polarization, $P_{e} . \tilde{A}_{F B}^{b}$, the asymmetry which is defined in Equation 1.70, and which is proportional to $A_{b}$ and $P_{e}$, can then be computed and $A_{b}$ extracted.

\section{Lifetime Tag}

The $Z^{0}$ decays into each fermion in Table 1.1 except for the $t$ quark, so the first step in the technique is to separate a sample of $Z^{0} \rightarrow b \bar{b}$ decays from the others, by taking advantage of the long lifetime and high mass of $B$ hadrons.

Track impact parameters to the interaction point (IP) have been used previously [135] to isolate enriched samples of $e^{+} e^{-} \rightarrow b \vec{b}$. The technique is often called a. "lifetime tag" because the average impact parameter of $B$ decay tracks is nearly. independent of the momentum of the parent $B$ hadron, but rather depends linearly on the average $B$ lifetime. $B$ hadrons can be separated from charmed hadrons since the latter decay more rapidly, and because $B$ hadrons have a larger mass, allowing for larger transverse components of the decay tracks' momenta. The parameters chosen for the tag are that three or more charged tracks miss the IP by more than $3 \sigma$ in the plane perpendicular to the beam axis, where $\sigma$ is the combined track measurement error and extrapolation uncertainty due to multiple scattering in the detector material. This tag is $61 \%$ efficient at a $Z^{0} \rightarrow b \bar{b}$ purity of $89 \%$ [134]. 


\section{Momentum-Weighted Track Charge}

The decay axis of the $Z^{0}$ is approximated using the thrust axis of the event [136], using LAC energy clusters ${ }^{\dagger}$. The task of finding the $b$ quark flight direction reduces to one of finding which of the two directions along the thrust axis the $b$ quark traveled, and which the $\bar{b}$. Techniques using leptons or charged kaons are effective at determining the sign of the $b$ quark [46][50], but the subset of $Z^{0} \rightarrow b \bar{b}$ decays for which these are present and confidently identified is not large. This analysis takes a more inclusive approach and uses a momentum-weighted sum of the charges of the tracks in each tagged event to identify the $b$ flight direction.

The event momentum-weighted charge $Q$ is defined to be

$$
Q=-\sum_{\text {tracks }} q_{i}\left|\vec{p}_{i} \cdot \vec{t}\right|^{\kappa} \operatorname{sgn}\left(\vec{p}_{i} \cdot \vec{t}\right),
$$

where $\vec{t}$ is the thrust axis, $q_{i}$ and $\vec{p}_{i}$ are the $i^{t h}$ track's charge and momentum, and $h$ is a parameter which may be adjusted to optimize the measurement sensitivity. This analysis chooses $\kappa=0.5$, a choice to be discussed in Section 6.4.1. The sign of $\vec{t}$ is chosen to make $Q$ positive, making $\vec{t}$ the estimator of the $b$ quark direction.

This technique of signing a parton's charge has a long history. It was first suggested by Feynman and Field [29] to distinguish between up- and down-type quark jets in hadronic collisions, and was named "jet charge." Momentum-weighted charge has been successfully used in $e^{+} e^{-}$experiments at lower energies at PEP and PETRA [137], TRISTAN [138], and more recently at LEP energies [139].

The SLD Monte Carlo suggests that the sign of $\vec{t}$ is chosen correctly $\sim 68 \%$ of the time, with better charge assignment when $\cos \theta$ is small, due to the CDC's acceptance. The correct-signing probability $P_{\text {correct }}$ can be expressed in terms of an "Analyzing Power" $(A P)$ :

$$
A P=P_{\text {correct }}-P_{\text {incorrect }}=2 P_{\text {correct }}-1 \sim 36 \% \text {. }
$$

$A P$ dilutes the asymmetry from its electroweak value, described in Section 1.4.1 down to its measured value. The analyzing power $A P$ depends on the details of $B$

tThe LAC provides a minimally biased estimate of the original direction of the partons emitted by the $Z^{0}$. Using tracks reconstructed by the CDC would bias the measured thrust axes toward the center of the detector, because of the loss of acceptance at high angles. Furthermore, the LAC allows reconstruction of neutral energy, reducing the uncertainty in the event axis. fragmentation at the $Z^{0}$, mixing and decay, as well as the detector response. Because of $\cos \theta$-dependent detector acceptance and radiative effects, $A P$ must be estimated as a function of $\cos \theta$ using a Monte Carlo with full simulation of these effects.

\section{Asymmetry and Fit}

Once the events are $b$-tagged and the signed direction of the $b$ in each one determined with momentum-weighted track charge, the left-right forward-backward asymmetry $\tilde{A}$ can be computed. Histograms binned in $\cos \theta_{T}$, the signed thrust axis, are accumulated separately with events produced with the left- and right-handed electron beams. Then

$$
\tilde{A}_{i}^{o b s}=\frac{N_{F L}^{i}-N_{B L}^{i}+N_{B R}^{i}-N_{F R}^{i}}{N_{F L}^{i}+N_{B L}^{i}+N_{B R}^{i}+N_{F R}^{i}}
$$

is calculated in each $\cos \theta$ bin, indexed with the letter $i$, with $L, R$ referring to the left- and right-handed beam helicity states, and $F, B$ referring to events with positive and negative $\cos \theta_{T}$.

This observed asymmetry must first be corrected for expectations of light-flavor contamination of the tag, an additive effect on the asymmetry.

$$
\tilde{A}_{i}^{\text {corr }}=\frac{\tilde{A}_{i}^{\text {obs }}}{\Pi_{i}}-\frac{1-\Pi_{i}}{\Pi_{i}} \tilde{A}_{i}^{\text {light }},
$$

where $\Pi_{i}$, the $b$-tag purity in each $\cos \theta$ bin, and $\tilde{A}_{i}^{\text {light }}$, the asymmetry of background events, are estimated from the Monte Carlo.

The corrected asymmetry $\hat{A}_{i}^{\text {corr }}$ is directly proportional to $P_{e} A_{b}$ in each bin of $\cos \theta_{T}$, and the coefficient is the analyzing power $A P$. The fit proceeds by finding the asymmetry in each bin of $\cos \theta_{T}$ in a pure Monte Carlo sample of $Z^{0} \rightarrow b \bar{b}$ events, with full modeling of $B$ fragmentation and decay, mixing, gluon radiation, and initial-state photon radiation ${ }^{\ddagger}$. The Monte Carlo asymmetries are compared with those in data, and an overall scale factor is determined for the Monte Carlo to determine the best fit. This scale factor constitutes a fully corrected measurement of $A_{b}$ using a binned fit.

The QED corrections mentioned in Section 1.5 are not applied to the measurement in this chapter because their effects are included in the Monte Carlo simulation. They will be necessary in Chapter 7, though 


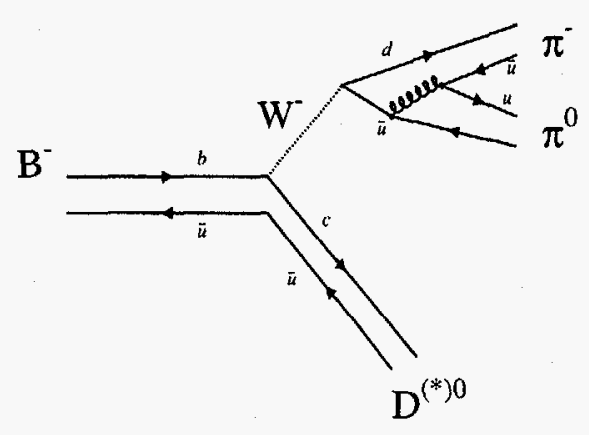

Figure 6.1: Feynman diagram of a typical $B$ decay, $B^{-} \rightarrow D^{\star 0} \pi^{+} \pi^{0}$.

\subsubsection{Why Momentum-Weighted Charge Works}

While momentum-weighted charge was originally proposed to distinguish between light-flavored jets [29], heavy-flavored decays of the $Z^{0}$ provide a much more optimal setting for its use. The reasons for this are twofold. First, the $B$ fragmentation function is extremely hard (see Figure 1.2 for a parameterization). The fraction of the beam energy a $B$ meson receives from a $Z^{0}$ decay is on average $\sim 70 \%$ [118][140], leaving relatively little energy for fragmentation tracks. When the $B$ loses a large fraction of energy to hard gluon radiation, the gluon jet has an average charge of zero [141] and develops no preference when momentum-weighting is applied.

The second reason is that the process of $B$ meson decay favors particles with charge correlated with that of the original $b$ quark with high momentum. In general, a $B$ decay contains $\dot{W}^{ \pm}$products, a charmed spectator, and perhaps some soft tracks from fragmentation of the spectator. The $W^{ \pm}$products, which come directly from the $B$ meson decay vertex, have a net charge of -1 when the $b$ quark itself has a charge of $-\frac{1}{3}$, and tend to have a high momentum in the laboratory, owing to the large mass of the recoiling spectator system and the available energy in the disintegration of the $B$. While the charmed spectator and its daughter products have a total charge that is anticorrelated with the original $b$, this is somewhat mitigated because the kaon into which the charmed hadron decays will have a charge that is positively correlated with the charge of the original $b$ quark. Because charmed mesons do not mix appreciably [142], the charge sign is not diluted through this step. Figure 6.2 shows the average charge in bins of $\ln \left(P_{t o t}\right)$ for particles which originate at the $B$ decay vertex, particles that originate at the cascade charm vertex (or vertices), and particles that originate at the $Z^{0}$ decay vertex. Because tracks with high momentum have more charge correlation with the originating quark, weighting the track charge with momentum improves the analyzing power.

An alternative approach to a track-charge analysis is to weight the charges with the rapidity of the tracks. This has been found to be about as effective as using momentum-weighting with $\kappa=0.5$.

\subsection{Experimental Results}

\subsubsection{Event Selection}

The SLD trigger has an efficiency $\epsilon \geq 96 \%$ [143] for accepting hadronic $Z^{\circ}$ decays. but it is also relatively efficient for backgrounds of various kinds. In addition, the $Z^{0}$ decays into final states which are not important for this analysis, such as the leptonic decays. The most serious background of the leptonic decay channels is the $\tau^{+} \tau^{-}$ final state. These events have a chance of passing the $b$-tag requirements because of the substantial lifetime of the $\tau$ lepton, and because the tracks have a high average momentum, which reduces the impact errors. 

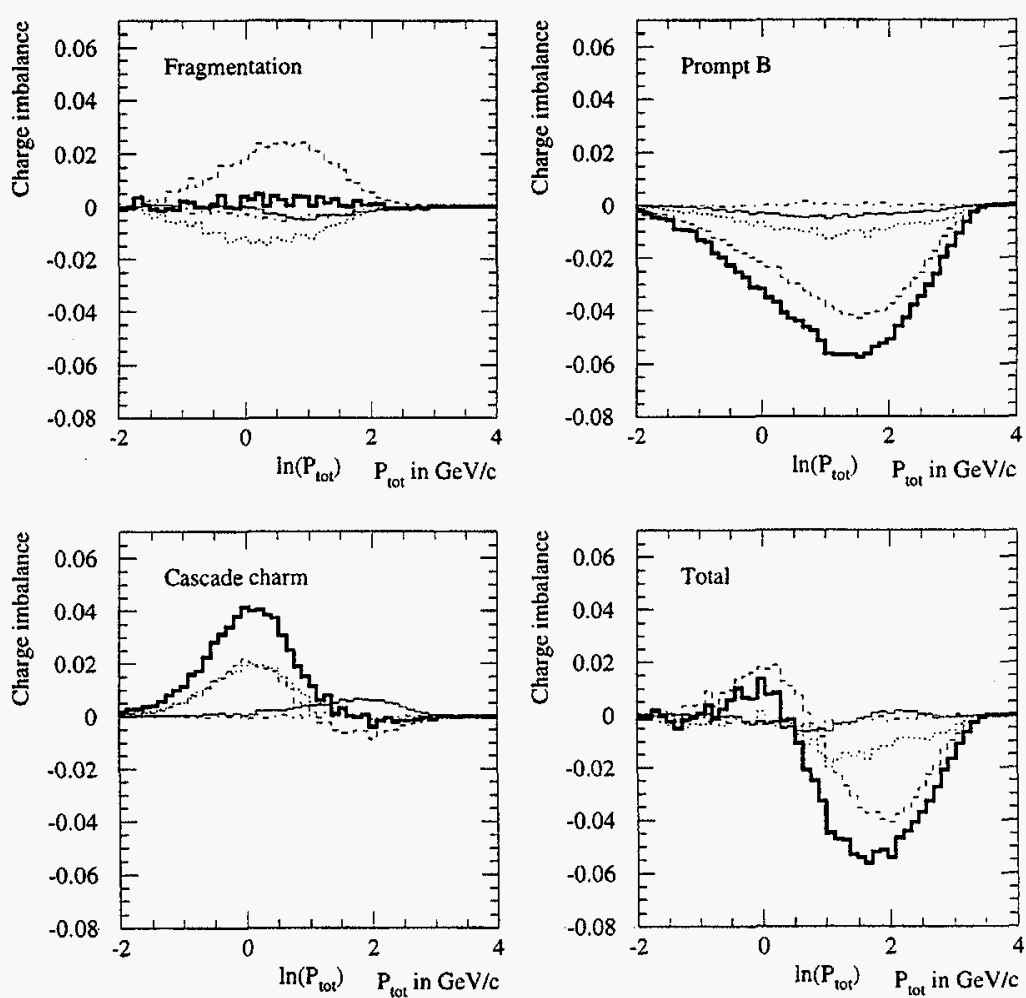

Figure 6.2: Average charge separation in bins of momentum for tracks from $B$ decay, cascade $D$ decay, and fragmentation, estimated with the SLD Monte Carlo. The histograms show the contributions from different species of $B$ hadrons - dashed: $B_{u}$, dotted: $B_{d}$, dot-dashed: $B_{s}$, and solid: $\Lambda_{b}$. The bold histogram is the sum over all species. The sign-correlated tracks from the $B$ decay vertex in general have more momentum than the oppositely-correlated tracks from the cascade charm decay vertex.

\subsubsection{Trigger and $Z^{0}$ Filter}

The first stage of event selection is the trigger applied online before the detector is read out. After the data are written to tape, they are quickly scanned by a filtering program, which applies loose calorimetric and tracking requirements on the events and writes a skimmed sample to tape. The combined trigger and filter efficiencies are estimated to be $93 \%$ [144][143]. Detailed descriptions of the trigger and filter are given in Appendix D.

During 1993, there was a flaw in one of the trigger requirements. A readout "veto" on triggers which incorporated tracking information, was configured improperly. The veto was designed to inhibit readout of the tracking chamber when the cell occupancy was too high, in order to reduce deadtime from accelerator backgrounds. The threshold was set too low from run 21573 to run 22553 , from here on called the "veto period." This period lasted from April 30, 1993, to June 17, 1993, and comprises roughly $2 / 5$ of the 1993 data sample. With this low threshold, the veto inhibited CDC readout on $\sim 20 \%$ of hadronic $Z^{0}$ events, with a bias against reading out highmultiplicity events. It is unfortunate for heavy flavor analyses because $Z^{0} \rightarrow b \bar{b}$ events have a higher average multiplicity than other hadronic decays and are therefore more likely to have been affected by this veto.

While the cell overflow veto is simulated in the Monte Carlo, it is more prudent to omit this data, as the vetoed sample's analyzing power may be different from that of the the non-vetoed sample.

\subsubsection{Analysis Requirements}

The filtered sample is too permissive a set to be useful for most analyses of the SLD data; only about $50 \%$ of it consists of hadronic $Z^{0}$ decays. A small fraction of it is mu pairs, tau pairs, and WAB's, with the remainder being combinations of various kinds of accelerator background. Each of the requirements listed in this section is designed to improve the purity of the hadronic $Z^{0}$ sample without introducing significant biases in the analysis results. Full detector displays of each of the different $Z^{0}$ decays can be seen in Figure 6.3. 

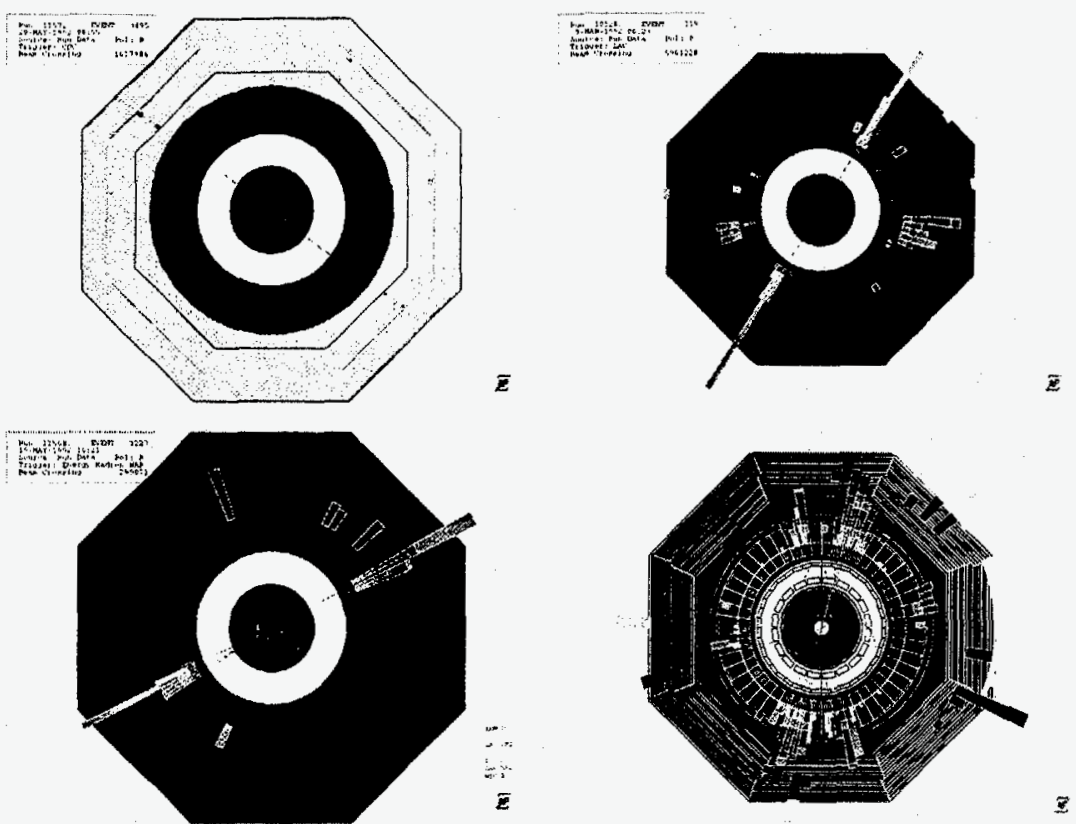

Figure 6.3: Different types of $Z^{0}$ decay. Clockwise from the upper left are $Z^{0} \rightarrow \mu^{+} \mu^{-}$, $Z^{0} \rightarrow e^{+} e^{-}, Z^{0} \rightarrow \tau^{+} \tau^{-}$, and $Z^{0} \rightarrow$ hadrons.

Hadronic decays of the $Z^{0}$ tend to have high charged multiplicities and large amounts of energy in the charged tracks. Leptonic $\left(e^{+} e^{-}\right.$or $\left.\mu^{+} \mu^{-}\right)$decays nearly always have charged multiplicities of 2 , while $Z^{0} \rightarrow \tau^{+} \tau^{-}$event decay multiplicities commonly reach up to 6 . The event selection will therefore rely on tracking information from the CDC.

\section{Track Selection}

In order to select events based on their charged tracks, one needs to require that the tracks themselves originate near the interaction region and be measured well. This
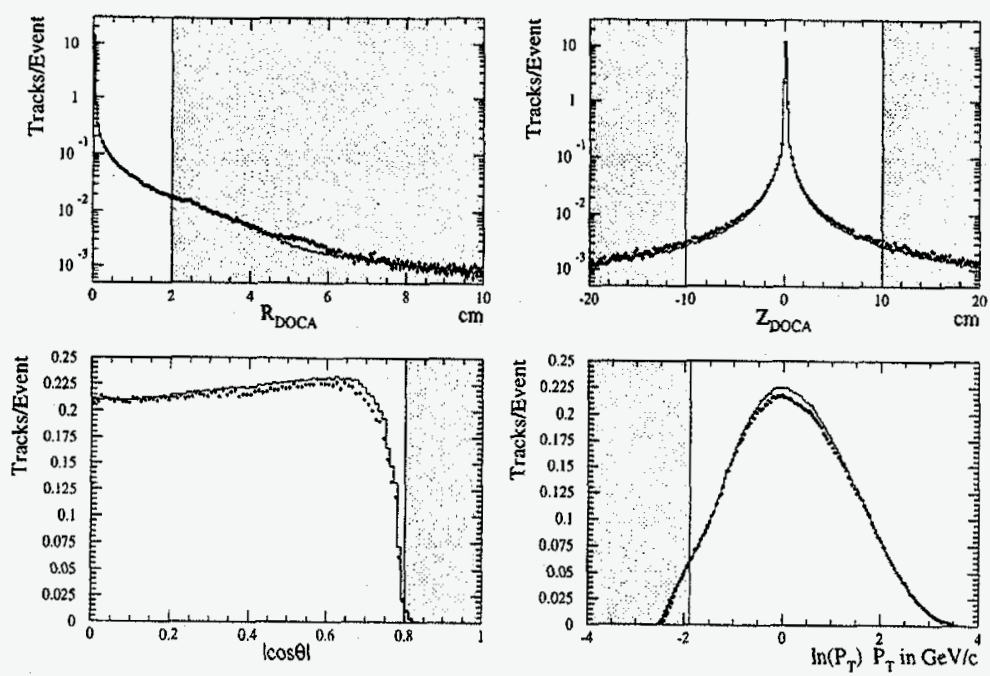

Figure 6.4: Distributions of the first four track selection variables (Equations 6.7-6.10). The data (points) are compared with the Monte Carlo (histogram). Shaded areas correspond to tracks which do not pass the selection criteria. In each plot, all track and event selection cuts have been applied except the one shown.

analysis requires that each track have

$$
\begin{aligned}
R_{D O C A} & <2.0 \mathrm{~cm}, \\
Z_{D O C A} & <10.0 \mathrm{~cm}, \\
|\cos \theta| & <0.8, \\
P_{t} & >150 \mathrm{MeV} / \mathrm{c}, \\
R_{\text {inner hit }} & <45 \mathrm{~cm}, \\
N_{\text {hits }} & >39 \text { of a possible } 80, \\
\chi^{2} / D O F & <5.0, \text { and } \\
P_{\text {tot }} & <50 \mathrm{GeV} / \mathrm{c},
\end{aligned}
$$



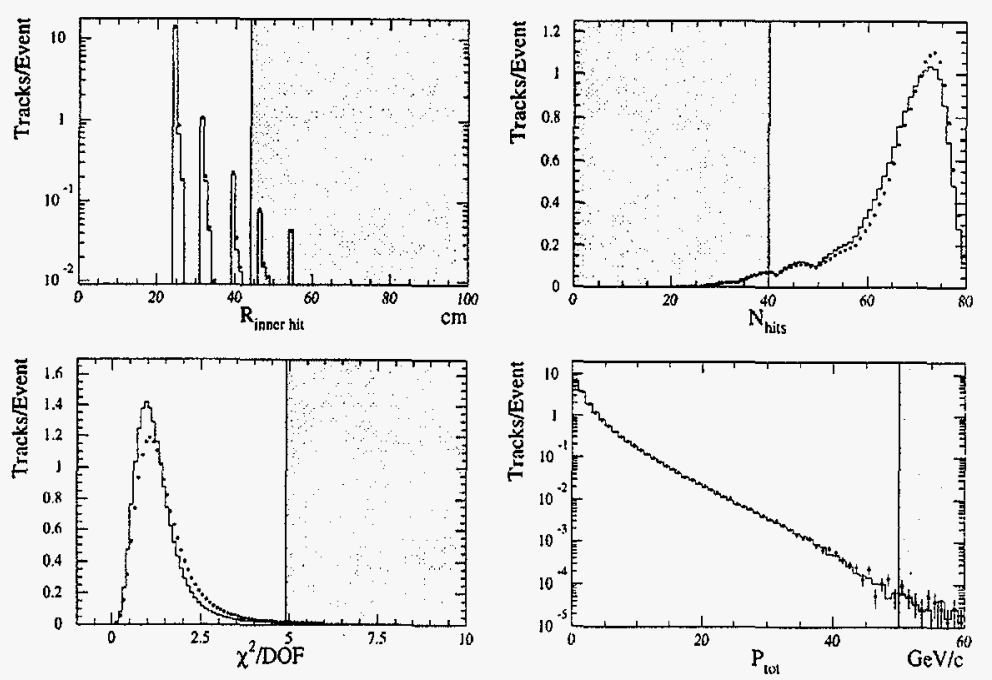

Figure 6.5: Distributions of the second four track selection variables (Equations 6.11-6.13). The data (points) are compared with the Monte Carlo (histogram). Shaded areas correspond to tracks which do not pass the selection criteria. In each plot, all track and event selection cuts have been applied except the one shown.

where $R_{D O C A}$ is the $\mathrm{x}-\mathrm{y}$ radius of the track at its point of closest approach to the beamline, $Z_{D O C A}$ is the $z$ coordinate of the same location, with $z=0$ within a few millimeters of the average IP position, $P_{t}$ is the momentum transverse to the beam axis, and $r_{\text {inner }}$ hit is the $\mathrm{x}-\mathrm{y}$ radius of the CDC hit closest to the beampipe. The purpose of the first two cuts above is to reject tracks that do not originate from the interaction point. One of the largest sources of extraneous charged tracks in the detector is the pair of tungsten synchrotron radiation masks M4, located at $z= \pm 40 \mathrm{~cm}$ from the IP [145]. Inelastic scattering in detector material of particles from $Z^{0}$ decay and stray particles from the beam also fall into this category. Figure 6.4 shows the $R_{D O C A}$ and $Z_{D O C A}$ distributions before cuts.

The acceptance of the CDC falls very quickly outside of $\left|\cos \theta_{t r a c k}\right|=0.75$, with
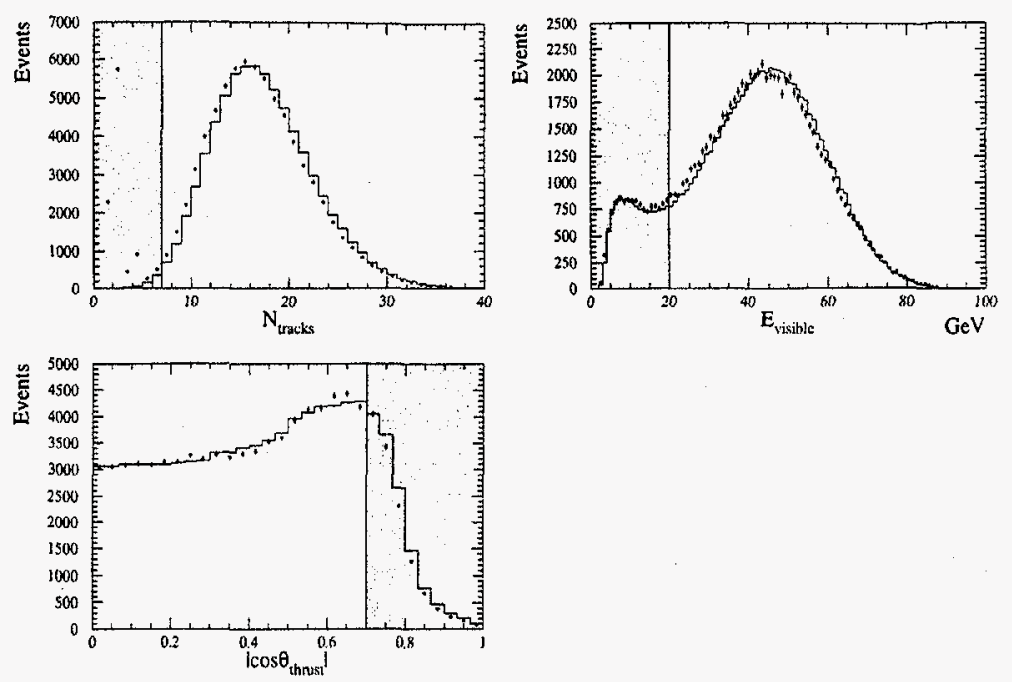

Figure 6.6: Distributions of the event selection variables (Equations 6.15-6.16). The data (points) are compared with the Monte Carlo (histogram). Shaded areas correspond to events which do not pass the selection criteria. In each plot, all track and event selection cuts have been applied except the one shown.

virtually no tracks left outside of $|\cos \theta|=0.8$, where an expected 7 out of 10 lavers are fully hit by the track. In this sense, the cut on $\cos \theta_{\text {track }}$ is almost not a cut at all. save for the fact that any track with $|\cos \theta|>0.8$ probably has not been reconstructed properly, or has come from some place other than the IP. This cut is also correlated with the cut on $N_{\text {hits }}$, and so contributes little.

The cut on $P_{t}$ serves two purposes. A large fraction of the beam-related background tracks have very low momentum transverse to the beam. Electromagnetic backgrounds such as Compton scatters from synchrotron radiation photons in the detector material have very low $P_{t}$ on this scale [145]. Tracks with $P_{t}$ under $\sim 100 \mathrm{MeV}$ also loop back into the tracking chamber and the track reconstruction may split them into multiple track segments. 
The radius of the closest hit requirement mainly rejects $K_{s}^{0}$ and $\Lambda$ decay product tracks that started midway through the CDC and may not be well measured. It also rejects the second halves of tracks that kinked because of decays or scatters and were split into two pieces. It is not a completely orthogonal cut to the $N_{\text {hits }}$ cut, which ensures that the track have enough measured points to determine the track parameters reliably. The $\chi^{2}$ cut attempts to reject tracks that have too many hits that were incorrectly assigned to them, and tracks that kinked but were not split into two tracks. If a track has a measured momentum greater than $50 \mathrm{GeV} / \mathrm{c}$, then it was probably mismeasured, perhaps due to a kink in the track, or hits that were taken from other tracks. The momentum measurement error of $45 \mathrm{GeV} / \mathrm{c}$ tracks with CDC-only information is $10.1 \mathrm{GeV}$, as given by Equation 6.29 ; the error drops by a factor of two when VXD information is added.

\section{Event Selection}

Once a set of clean tracks has been found, events are selected based on it. The requirements for this analysis are

$$
\begin{aligned}
N_{\text {tracks }} & \geq 7, \\
E_{\text {visible }} & \geq 20 \mathrm{GeV}, \text { and } \\
\left|\cos \theta_{\text {thrust }}\right| & <0.7,
\end{aligned}
$$

where $E_{\text {visible }}$ is the sum of the energies in charged tracks passing the above criteria. assuming each has the mass of a pion, and $\theta_{\text {thrust }}$ is the polar angle of the thrust axis found using energy clusters in the LAC. Because the $N_{\text {tracks }}$ requirement is based on selected tracks, it is effective in eliminating beam-related background triggers in which no tracks come from the interaction point. The cut of 7 tracks was chosen to remove nearly all of the $Z^{0} \rightarrow \tau^{+} \tau^{-}$background without too significant a loss of hadronic efficiency. The cut at 7 tracks requires at least one of the taus in the event to have a five-prong decay (the other must have at least three prongs in its decay), or for some tracks to be misreconstructed. The five-prong branching fraction of taus is $(1.25 \pm 0.24)^{-3}$ [146], and the three-prong fraction is $(14.38 \pm 0.24) \%$, and $\Gamma\left(Z^{0} \rightarrow \tau^{+} \tau^{-}\right) / \Gamma\left(Z^{0} \rightarrow\right.$ hadrons $) \simeq 4.8 \%$, so the contamination is expected to be very small. A Monte Carlo study indicates that the efficiency of the event selection for $Z^{0} \rightarrow \tau^{+} \tau^{-}$events is $(0.77 \pm 0.09) \%$, and that therefore the $Z^{0} \rightarrow \tau^{+} \tau^{-}$contamination is $(0.09 \pm 0.01) \%$. Similar Monte Carlo studies indicate that the selection efficiency for WABs is $0.02 \%$ and the selection efficiency for $2 \gamma$ events is $<0.02 \%$. Dimuon events are expected to be less efficient than WAB's, owing to less final-state radiation and bremsstrahlung in the detector material to convert into $e^{+} e^{-}$pairs.

The visible energy cut is also designed to help eliminate beam-related backgrounds and $2 \gamma$ events, as tracks within the detector acceptance for these triggers tend to be very soft. A comparison of this variable in data and Monte Carlo with all other selection cuts applied is shown in Figure 6.6. The cut on the polar angle of the thrust axis is applied to improve the quality of the tagged event sample. Because the coverage of the vertex detector extends only to tracks with $|\cos \theta|<0.75^{\S}$ [112], any event with a LAC thrust axis far beyond that is likely to have suffered severe QCD radiation or have large calorimeter backgrounds.

The efficiency of these cuts can be estimated from Monte Carlo to be $\sim 60 \%$, with the main inefficiency in this selection coming from the cut on $\left|\cos \theta_{\text {thrust }}\right|$. When the events are analyzed, only the tracks passing the selection criteria are used. A total of 15,858 hadronic events pass event selection in the 1993 sample (omitting the veto period). The total for $1994-1995$ is 59,430 events.

\subsubsection{B Tagged Sample}

To take advantage of the large mass and long lifetime $(\sim 1.5 \mathrm{ps})$ of the $B$ hadrons and of the fine resolution of the SLD tracking chambers, this analysis employs a 2dimensional signed impact parameter tag to identify $Z^{0} \rightarrow b \bar{b}$ events. This tag has been described in detail elsewhere [134][147]. The tracks and the beam spot are projected into the plane perpendicular to the beam axis for purposes of the tag. Because the impact parameter resolution is dependent on the momentum of the track and its dip angle, which together determine how severe the multiple scattering is expected

\footnotetext{
${ }^{5}$ While the innermost layer of the VXD extends out to $|\cos \theta|<0.85$, tracks are not guaranteed to hit it. One-hit VXD coverage extends roughly to $|\cos \theta|=0.80$.
} 
to be in the beampipe and detector material, this analysis uses the normalized impact parameter $b / \sigma_{b}$, the impact parameter divided by its measurement error, as the indicator of significance.

The sign of the impact parameter $b$ is chosen with the aid of jets formed from reconstructed tracks using the JADE algorithm [148], with $y_{\text {cut }}=0.02$. A low $y_{\text {cut }}$ is chosen to prevent combining distinct jets together into larger ones with less angular resolution.

If the 2-dimensional projection of a track intersects its jet's axis on the same side of the IP as the track's flight path, then the impact parameter is signed positive. In the converse case, the track appears to have originated on the far side of the beam spot, and it is given a negative impact parameter. Because the boost of the heary mesons is large at the $Z^{0}$, virtually no tracks at all will originate on the far side of the IP relative to the direction in which they travel. The negative side of the impact parameter distribution is therefore a measure of the resolution and correct-signing of the impact parameters alone.

In order to ensure that the tracks used in the tag have well-measured impact parameters, additional requirements are applied to the tracks. They are:

$$
\begin{aligned}
N_{V X D h i t s} & \geq 1, \\
b & <3 \mathrm{~mm}, \text { and } \\
\sigma_{b} & <250 \mu \mathrm{m},
\end{aligned}
$$

where $N_{V X \text { Dhits }}$ is the number of clusters in the Vertex Detector associated with the track, and $b$ is the impact parameter. Distributions of these variables are found in Figure 6.7. In addition, tracks identified as a decay product of a $K^{0}$ or a $\Lambda$, or the product of a $\gamma$ conversion in detector material, are omitted from the tag analysis. These additional requirements are not applied for the tracks used in the momentumweighted charge, as the loss of even one track in the selection cuts due to a scatter or a mislink to VXD hits will reduce the probability that the event will be signed correctly.

The normalized impact parameter distribution is shown in Figure 6.8 for 1994 data and Monte Carlo. Figure 6.9 shows a breakdown by flavor of the normalized
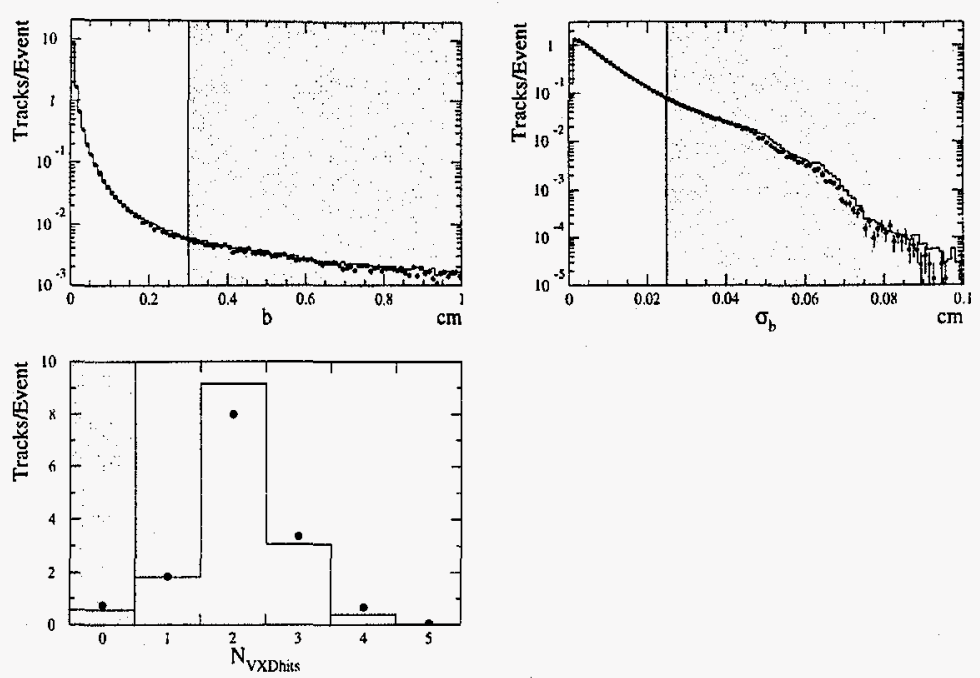

Figure 6.7: Distributions of vertexing-quality track cut variables. The data are shown as points and the Monte Carlo simulation values are shown as histograms. Data in the shaded regions are rejected by the track selection for purposes of $B$-tagging only.

impact parameter distributions as estimated in the Monte Carlo. No extra smearing of the Monte Carlo distributions has been applied, although because the data and Monte Carlo multiplicity distribution of Figure 4.4 do not match, an ad hoc tracking inefficiency has been applied to the Monte Carlo.

To tag an event, three tracks or more are required to have normalized impact parameters $b / \sigma_{b}>3.0$. An event which has many tracks with highly significant impāct parameters is shown in Figure 6.10. The performance of this tag can be seen in Figure 6.11, where the composition of the Monte Carlo is shown as a function of the number of such tracks. The total rate as a function of the number of significant tracks can be compared between data and Monte Carlo. The estimated efficiency of the $\operatorname{tag}$ is $61 \%$ and the purity is $89 \%$, with nearly all of the contamination, $10 \%$, 

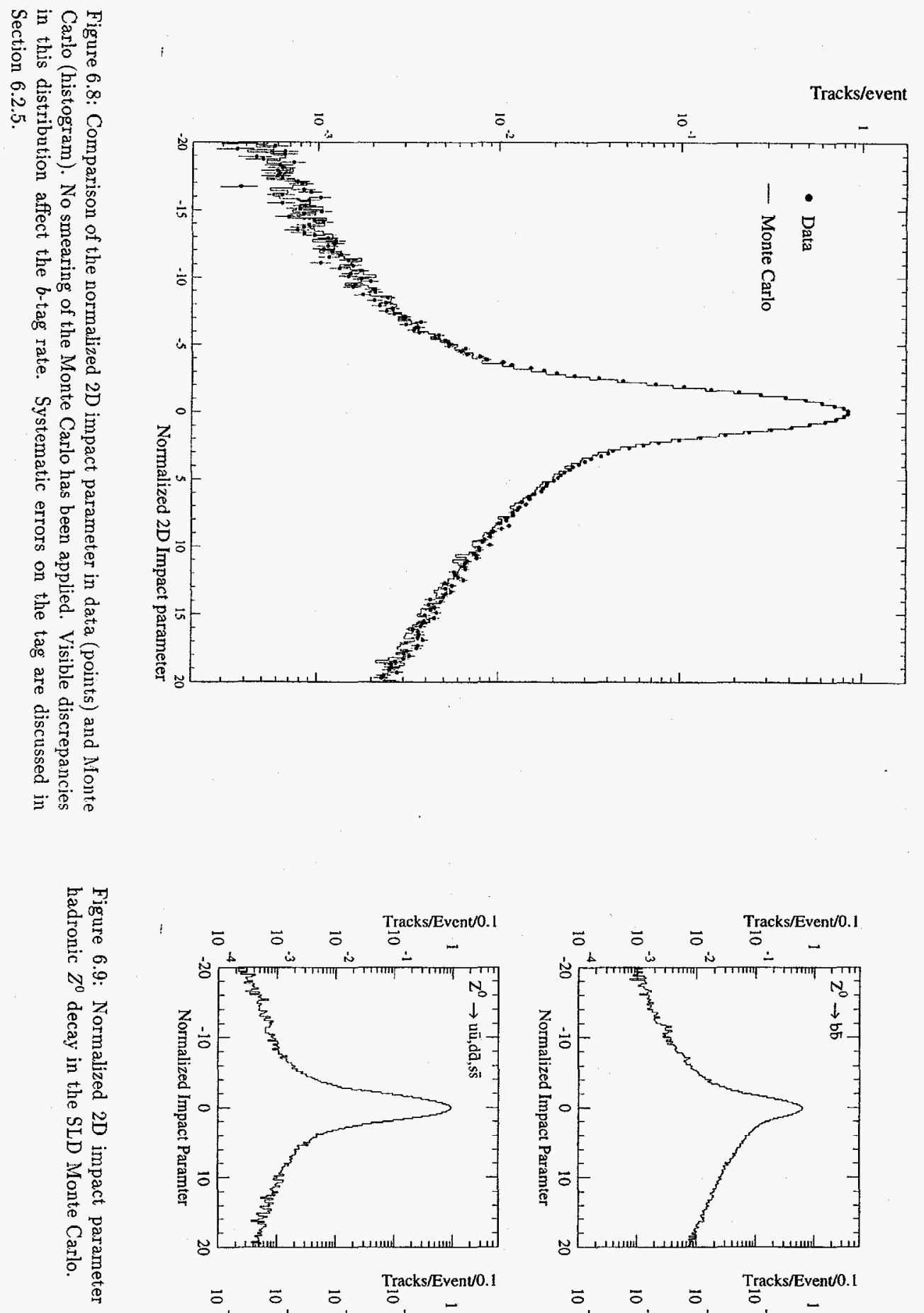



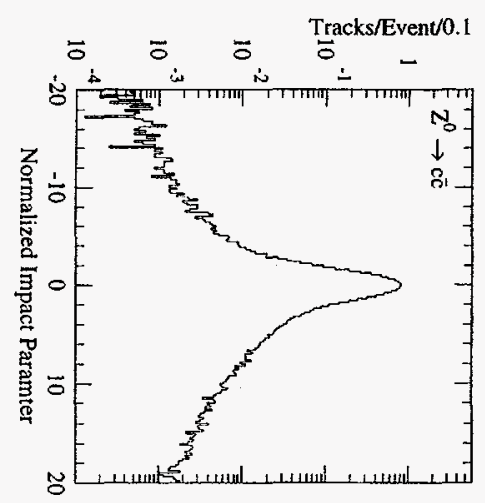




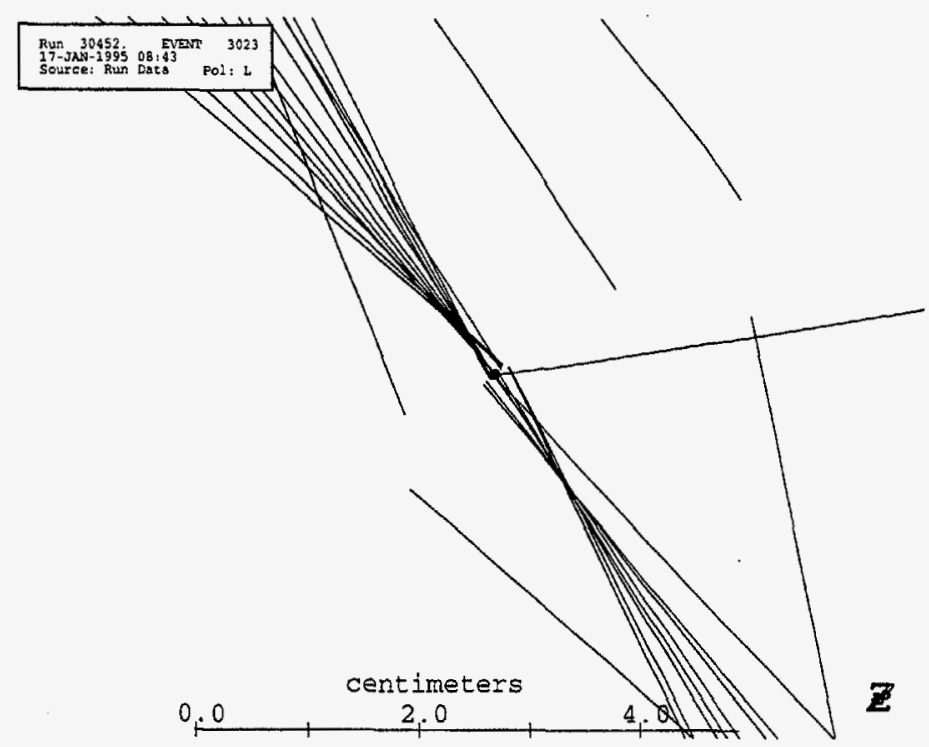

Figure 6.10: An event tagged with the normalized 2-D impact parameter tag. In this case, there are enough tracks with significant impact paramters to distinguish at least one displaced vertex in each hemisphere. The beam spot lies in the center of the square box with an error of $6 \mu \mathrm{m}$ in both directions in this projection.

coming from $Z^{0} \rightarrow c \bar{c}$ events, and only $1 \%$ from $Z^{0} \rightarrow u d s$ events.

The fraction of events tagged in the data is $(15.96 \pm 0.13) \%$ for events retained by the hadronic event selection. A Monte Carlo study indicates that $(7.3 \pm 3.2) \%$. $Z^{0} \rightarrow \tau^{+} \tau^{-}$decays which pass event selection also pass the 2-D impact parameter tag. The total estimate of the fraction of $Z^{0} \rightarrow \tau^{+} \tau^{-}$events in the tagged sample is $(0.04 \pm 0.02) \%$.

The tagged event sample consists of 2,504 events in the 1993 data set, and 9,241 events in the 1994-1995 data set.

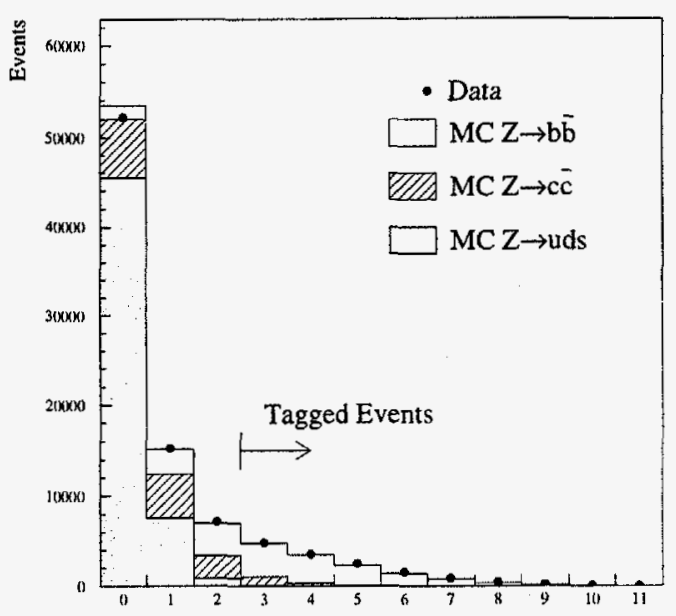

Number of Tracks with $\mathrm{b} / \sigma_{\mathrm{b}}>3$

Figure 6.11: Performance of the 2-Dimensional Normalized Impact-Parameter Tag

\subsubsection{Systematic Uncertainties on the B Tag}

The performance of the 2-D normalized impact parameter tag is affected by the properties of the SLD tracking systems and by the underlying processes of $B$ fragmentation and decay; additional model dependence comes from the simulation of $Z^{0} \rightarrow c \bar{c}$ events and gluon splitting.

Because the Monte Carlo simulation of the tag provides the estimation of the purity of the $Z^{0} \rightarrow b \bar{b}$ sample and also the composition of the contaminating fractions. it is crucial that the simulation be correct, and that conceivable deviations between the model and the true processes be well understood. In addition, the sensitivity of the analysis to the model parameters which may not be well constrained must be evaluated, and errors propagated through the $A_{b}$ measurement.

The only property of the tag to which this analysis is sensitive is the composition. 
Table 6.1: Systematic errors of the 3-tracks at $3 \sigma$ 2-D impact parameter tag, after Reference [134].

\begin{tabular}{|c|c|c|c|}
\hline Detector Modeling & & $\delta \epsilon_{b} / \epsilon_{b}(\%)$ & $\delta \Pi_{b} / \Pi_{b}(\%)$ \\
\hline $\begin{array}{c}\text { Tracking \& } \\
\text { Linking efficiency }\end{array}$ & & 2.9 & 0.32 \\
\hline Tracking resolution & & $<0.1 \%$ & $<0.1 \%$ \\
\hline $\begin{array}{c}\text { Trigger \& } \\
\text { Event Selection }\end{array}$ & & 0.5 & 0.06 \\
\hline Beam position tails & & 0 & 0.5 \\
\hline Physics Modeling & Variation & & \\
\hline$B$ lifetimes & $\begin{array}{l}\tau_{B_{\text {mesor }}}=1.55 \pm 0.10 \mathrm{ps} \\
\tau_{B_{\text {baryan }}}=1.10 \pm 0.30 \mathrm{ps}\end{array}$ & 2.6 & 0.29 \\
\hline$b$ fragmentation & Peterson $\left\langle x_{e}\right\rangle=0.695 \pm 0.021$ & 2.2 & 0.24 \\
\hline$b$ fragmentation & Bowler vs. Peterson at $\left\langle x_{e}\right\rangle=0.695$ & 0.2 & 0.02 \\
\hline$b$ baryon production & $(8.9 \pm 3.0) \%$ & 0.6 & 0.07 \\
\hline$B$ decay to $D^{+}$ & ( $\pm 6 \%$ absolute) & 0.3 & 0.03 \\
\hline$B$ decay multiplicity & \pm 0.25 tracks per $B$ decay & 2.2 & 0.24 \\
\hline$B$ model & Phase space vs. Tuned JETSET 6.3 & 0.7 & 0.08 \\
\hline$c$ fragmentation & Peterson $\left\langle x_{e}\right\rangle$ for $D^{*}=0.501 \pm 0.025$ & 0 & 0.5 \\
\hline$c$ fragmentation & Bowler vs. Peterson at $\left\langle x_{e}\right\rangle=0.501$ & 0 & 0.1 \\
\hline$c$ decay to $D^{+}$ & $\pm 5 \%$ absolute & 0 & $<0.1 \%$ \\
\hline$c$ decay multiplicity & & 0 & 0.9 \\
\hline$s$ production & $s \bar{s}$ popping $\pm 10 \%$ & 0 & 0.3 \\
\hline $\begin{array}{l}\text { vds decay } \\
\text { multiplicity }\end{array}$ & \pm 0.3 tracks & 0 & 0.1 \\
\hline$g \rightarrow b \bar{b}$ splitting & $\pm 50 \%$ of JETSET & 0 & 0.5 \\
\hline$g \rightarrow c \bar{c}$ splitting & $\pm 50 \%$ of JETSET & 0 & 0.3 \\
\hline$\Gamma\left(Z^{0} \rightarrow c \bar{c}\right)$ & $R_{c}=0.171 \pm 0.017$ & 0 & 1.0 \\
\hline Jet axis modeling & JADE $y_{\text {cut }}$ varied from 0.01 to 0.10 & 0.8 & .09 \\
\hline Total & & $5.2 \%$ & $1.8 \%$ \\
\hline
\end{tabular}

Some sensitivity to the efficiency comes from its effect on the purity, through the following relation

$$
\Pi_{b}=\frac{R_{b} \epsilon_{b}}{R_{b} \epsilon_{b}+R_{c} \epsilon_{c}+\left(1-R_{b}-R_{c}\right) \epsilon_{u d s}}
$$

where $\Pi_{b}$ is the tag purity, and $\epsilon_{b}$ is the tag efficiency. Systematic uncertainties for the efficiency and composition of the 2-D normalized impact parameter tag have been estimated in reference [134] and are presented in Table 6.1, with their resulting effects on just the $B$ tag purity.

The discrepancies visible in Figures 6.8 and 6.11 result in a $\sim 5 \%$ higher tag fraction in the data than in the Monte Carlo. Given that largest errors in the tag rate come from uncertainties in $\epsilon_{b}$ and not the purity, these discrepancies are of less concern for the measurement of $A_{b}$.

Because the tag is nearly $90 \%$ pure in $Z^{0} \rightarrow b \bar{b}$ decays and nearly all of the contamination comes from $Z^{\circ} \rightarrow c \bar{c}$ events, the largest contributions come from uncertainties in $R_{c}$ and the modeling of charm decays. Uncertainties in the $B$ tagging efficiency are scaled down by roughly 0.1 in their effect on uncertainties on the $B$ purity.

\subsubsection{Momentum-Weighted Charge Distributions}

Because the binned asymmetry fit technique uses the Monte Carlo to estimate how often the momentum-weighted track charge of Equation 6.2 signs the events properly: it is important to verify that the Monte Carlo reproduces the distribution of that variable. The comparison must be made with $|Q|$, where $Q$ is defined in Equation 6.2. The distribution of a signed $Q$ would introduce the polarization and $A_{b}$; the unsigned distribution depends only on the details of fragmentation, decay, and detector response.

The $Q$ distribution, shown in Figure 6.12, fits to a Gaussian with zero mean, with $\chi^{2}=43.4$ for the data, and $\chi^{2}=56$ for the Monte Carlo, both with 28 degrees of freedom?. The width of the $Q$ distribution in data is $(4.187 \pm 0.025)(\mathrm{GeV})^{\frac{1}{2}}$, and that of the data is $(4.330 \pm 0.015)(\mathrm{GeV})^{\frac{1}{2}}$. The property that $Q$ is nearly Gaussian will aid in the calibration of the analyzing power in the next chapter.

"In fact, the distribution of $Q$ is not expected to be perfectly Gaussian even if the underiving momentum-weighted charge distributions are. This point will be brought up in detail in Chapter 7 . 


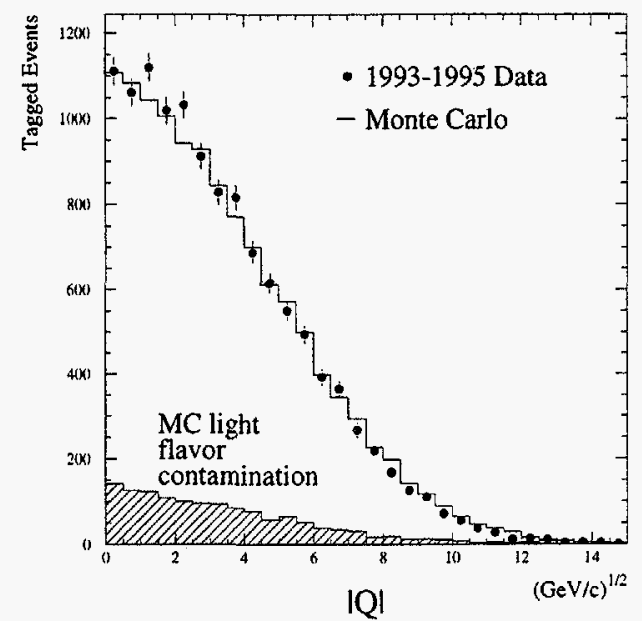

Figure 6.12: Momentum-Weighted Charge comparison of data and Monte Carlo for tagged events. The momentum-weighting exponent $\kappa$ is set to 0.5 . The Monte Carlo distribution is broken down into a signal $\left(Z^{0} \rightarrow b \bar{b}\right)$ subsample, shown as a histogram, and contamination from light flavored hadronic decays, shown hatched. Only the absolute value is shown, so as not to bias the comparison with asymmetry-dependent information.

A second quantity can be formed which yields information about the material in the detector. The quantity

$$
Q_{\text {sum }}=\sum_{\text {tracks }} q_{i}\left|\vec{p}_{i} \cdot \vec{t}\right|^{\kappa}
$$

ignores whether a track went into the forward hemisphere or the backward hemisphere, and therefore contains no information about the measured asymmetry. Furthermore, it is a signed quantity, and its average value is an indication of the contribution of extra positive charged tracks due to interactions with detector material of the particles originating at the $Z^{0}$ decay. This distribution, shown in Figure 6.13, is also nearly Gaussian with a mean of $0.088 \pm 0.033(\mathrm{GeV})^{\frac{1}{2}}$ and a width of $3.654 \pm 0.024$

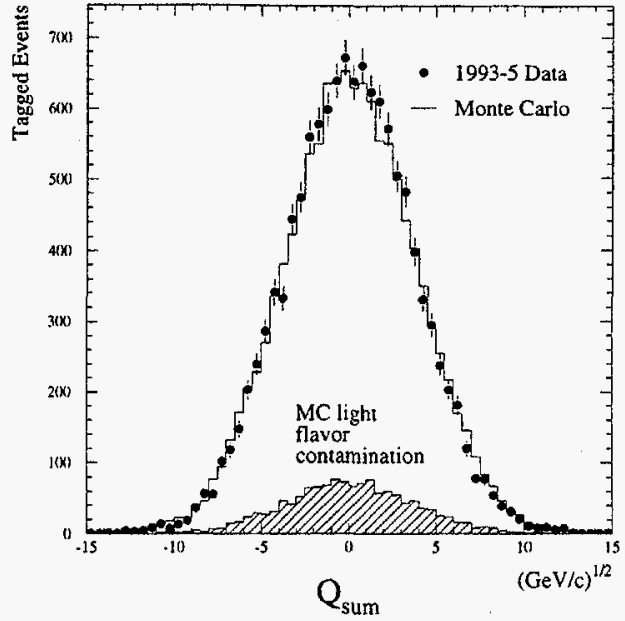

Figure 6.13: Distribution of $Q_{\text {sum }}$ for tagged events, compared between data and Monte Carlo. The Monte Carlo $Z^{0} \rightarrow b \bar{b}$ contribution is shown in the clear histogram, while the light-flavor $Z^{0}$ decays are shown in the hatched histogram.

$(\mathrm{GeV})^{\frac{1}{2}}$ in the data, and a mean of $0.010 \pm 0.020(\mathrm{GeV})^{\frac{1}{2}}$ and a width of $3.774 \pm 0.014$ $(\mathrm{GeV})^{\frac{1}{2}}$ in the Monte Carlo. The $\chi^{2}$ values are 48.4 and 70.1 for 51 degrees of freedom in the data and Monte Carlo, respectively. This width will become important later in the maximum-likelihood fit, as it carries information about the analyzing power.

\subsubsection{Asymmetry Fit}

Once the momentum-weighted charge $Q$, defined in Equation 6.2, has been calculated for the tagged events and the thrust axis signed, the left-right forward-backward asymmetry may be formed. To demonstrate the large asymmetry present in the $Q$ signed data sample, the events are binned in $\cos \theta$ separately for events produced when the electron beam was left- and right-handed polarized, shown in Figure 6.14. 


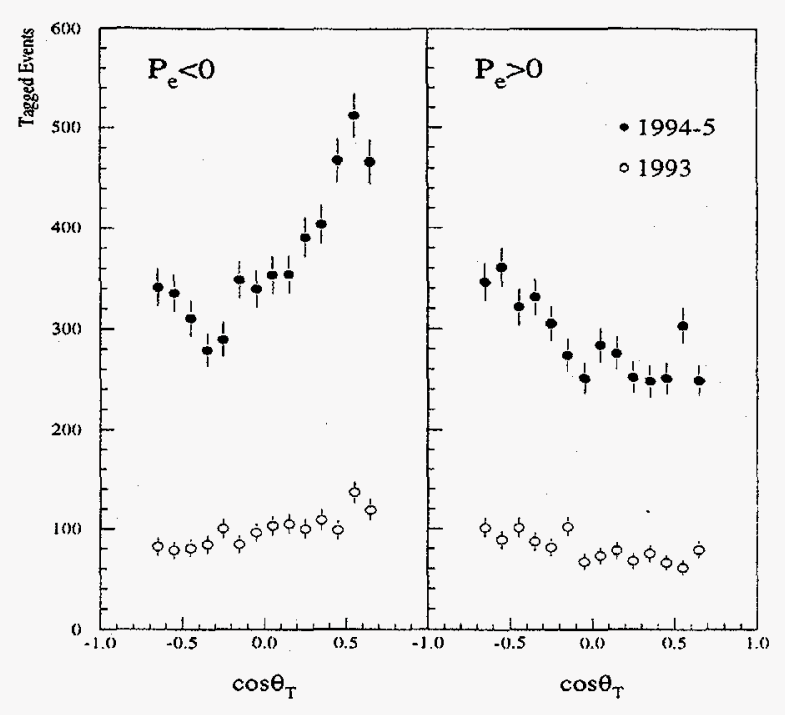

Figure 6.14: Calorimetric thrust axis $\cos \theta$, signed with momentum-weighted track charge. binned separately for events created with the left-handed and right-handed $e^{-}$beams. The effects of the increased polarization from $63 \%(1993)$ to $77 \%(1994-5)$, and the larger data sample in 1994-5, are visible.

The Standard Model prediction of the forward-backward asymmetry is larger for the events produced with the left-handed beam than for the right-handed beam. One may also see the effects of $A_{L R}$ - the $Z^{0}$ production cross-section is larger for the left-handed electrons than for right-handed electrons. There are 6,565 tagged events produced when the $e^{-}$beam was left-handed and 5,180 tagged events produced when the $\bar{e}^{-}$beam was right-handed in the 1993-1995 data sample.

The left-right forward-backward asymmetry is then formed as prescribed by Equation 6.4. The Monte Carlo is used to estimate the contamination fraction and asy'mmetry in each bin of $\cos \theta$. The contamination asymmetry is then subtracted from the data asymmetry according to Equation 6.5. Typically, the background asymmetry has a value of -0.75 times the signal asymmetry. The sign is negative because the

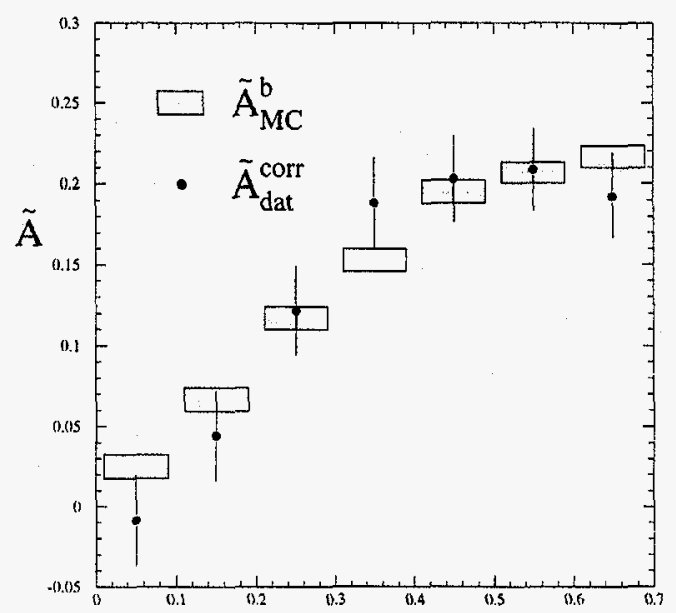

$\left|\cos \theta_{\mathrm{T}}\right|$

Figure 6.15: Binned fit of the $\mathrm{MC}$ asymmetry to the data asymmetry, taking into account $\cos \theta_{T}$-dependent effects on the analyzing power. The Monte Carlo statistical error is indicated by the shaded regions.

background is dominated by $Z^{0} \rightarrow c \bar{c}$ events, and the value is less than unity because $A_{c}<A_{b}$, and because $Z^{0} \rightarrow c \vec{c}$ events are expected to have less analyzing power on average than $Z^{0} \rightarrow b \bar{b}$ events. This results in a bin-by-bin correction of approximately $21 \%$. The correctness of the modeling necessary to subtract the contamination properly may be tested by repeating the entire analysis with stronger and weaker tags. the results of which are described in Section 6.4.2.

There remains a large amount of ordinary physics and detector processes which affect the measurement of $A_{b}$ with momentum-weighted track charge, and therefore must be accounted for with a detailed simulation. These effects include tracking efficiency, $B^{0}-\bar{B}^{0}$ mixing, charge dilution from the properties of the $B$ decay process. 
fragmentation tracks, stray particles produced by interactions with the detector material, thrust-axis resolution, radiation of hard gluons, heavy quark pair splitting from gluons, and initial state radiation. These effects are simulated by the SLD Monte Carlo, which incorporates a full implementation of JETSET 7.4, with the decays of $B$ mesons taken care of by the CLEO $B$-decay model. The decay tables of the $D$ mesons have also been altered from the default JETSET values in order to agree better with available measurements, and are presented in Chapter 5 . The average $B$ mixing parameter $\chi_{B}$ is 0.130 in the SLD Monte Carlo, with near saturation of $B_{s}$ mixing, and $\chi_{d}=0.180$ for the $B_{d}$ meson alone.

While all of the known "ordinary" physics effects which may dilute the observed asymmetry are modeled in the Monte Carlo, it is important to evaluate how much of an effect each is contributing to the answer, and to estimate the uncertainties in each, which will be the subject of the next section. Another important feature of most of these corrections is that they depend on the polar angle. Because the underlying asymmetry also has a dependence on polar angle given by Equation 1.72, and therefore the different bins in $\cos \theta$ receive different weights in the fit, these effects must be taken into account separately in each bin of $\cos \theta$. Fortunately, though, the asymmetry in each bin scales proportionally with $P_{e} A_{b}$, and therefore a simple scaling fit of the Monte Carlo asymmetry to the data asymmetry is justified. The Monte Carlo is then run at $P_{e}= \pm 100 \%$ and $A_{b}=1.0 \|$, and the measured $A_{b}$ is given by

$$
A_{b}=\frac{1}{\left\langle P_{e}\right\rangle_{\mathcal{L}}}\left(\frac{\sum_{i} \tilde{A}_{i}^{\mathrm{corr}} \tilde{A}_{M C, i}^{b} / \sigma_{i}^{2}}{\sum_{i}\left(\tilde{A}_{M C, i}^{b}\right)^{2} / \sigma_{i}^{2}}\right)
$$

where $\left\langle P_{e}\right\rangle_{\mathcal{L}}$ is the luminosity-weighted electron beam polarization, and $\tilde{A}_{M C, i}^{b}$ is the left-right forward-backward asymmetry calculated in bin $i$ of $\cos \theta$ in the Monte Carlo. for a pure sample of $Z^{0} \rightarrow b \bar{b}$ events that have been tagged. Typically $\tilde{A}_{M C, i}^{b}$ is $\sim 36 \%$ of the raw cross-section asymmetry in its bin, corresponding to a correct-sign fraction

\footnotetext{
"In practice, the Monte Carlo is run with $A_{b}^{M C}=0.9357$ and the resulting fit value of $A_{b}$ is multiplied by $A_{b}^{M C}$. In addition, the SLD Monte Carlo is generated with equal quantities of lefthanded events and right-handed events. This unphysical situation affects the calculation of $\bar{A}$ in the $\mathrm{MC}$, and therefore must be adjusted to reflect $A_{L R} \simeq 0.15$ by removing a fraction of the right-handed events, as described in Section 5.2 .
}

of $\sim 68 \%$. The bins are weighted by the statistical error on the data measurements $\sigma_{i}$, which is given by a binomial error expression

$$
\sigma_{i}=2 \sqrt{\frac{\left(N_{F L}^{i}+N_{B R}^{i}\right)\left(N_{B L}^{i}+N_{F R}^{i}\right)}{\left(N_{F L}^{i}+N_{B R}^{i}+N_{B L}^{i}+N_{F R}^{i}\right)^{3}}},
$$

in each bin of $\cos \theta$. The statistical error used in the fit is magnified by the correction for light-flavor contamination as prescribed by Equation 6.5:

$$
\sigma_{\tilde{A}^{c o r r}}^{i}=\frac{\sigma_{\tilde{A}^{o b s}}^{i}}{\Pi_{b}^{i}}
$$

also calculated separately in each bin of $\cos \theta$. The statistical error on the fit for $A_{b}$ in Equation 6.22 is given by

$$
\left.\delta A_{b} \text { (stat. }\right)=1 /\left(\left\langle P_{e}\right\rangle_{\mathcal{L}} \sqrt{\sum_{i}\left(\tilde{A}_{M C, i}^{b}\right)^{2} / \sigma_{i}^{2}}\right) .
$$

Using a luminosity-weighted polarization of

$$
\left\langle P_{\epsilon}\right\rangle_{\mathcal{L}}=0 . \overline{\mathrm{T}} 39
$$

averaged over 1993-1995 data (omitting the 1993 veto period), the value fit by this procedure is

$$
A_{b}=0.828 \pm 0.054 \text { (stat.) }
$$

The statistical error scales as $1 /\left(P_{e} \cdot A P \cdot \sqrt{N}\right)$, with much of the magnification coming from $1 / A P$. Gains in sensitivity can often be made more quickly by improving $A P$ than by collecting more data. This is the strategy taken by the semileptonic analyses and the inclusive kaon analysis, although the efficiency of the impact-parameter tag compensates in this case for the loss of analyzing power incurred by using moment umweighted track charge.

\subsection{Systematic Error Analysis}

Systematic errors on the measurement of $A_{b}$ arise from parts of the analysis in which a model is used to interpret the data. The most important of these is the binned 
Table 6.2: Details of the binned asymmetry fit for $A_{b} . \tilde{A}_{M C}^{b}$ is tabulated as if $A_{b}=P_{e}=1.00$ in the Monte Carlo; $\tilde{A}_{M C}^{\text {light }}$ is reported at the correct luminosity-weighted polarization and assumes Standard Model couplings, so that it may be directly used in Equation 6.5.

\begin{tabular}{|ccccc|}
\hline $\cos \theta_{T}$ & $\tilde{A}^{\text {obs }}$ & $\tilde{A}_{M C}^{\text {light }}$ & $\Pi_{b}$ & $\tilde{A}^{\text {corr }}$ \\
\hline $0.0-0.1$ & $-0.011 \pm 0.025$ & -0.032 & 0.887 & $-0.009 \pm 0.028$ \\
$0.1-0.2$ & $0.030 \pm 0.024$ & -0.064 & 0.879 & $0.043 \pm 0.028$ \\
$0.2-0.3$ & $0.106 \pm 0.025$ & -0.030 & 0.898 & $0.121 \pm 0.028$ \\
$0.3-0.4$ & $0.151 \pm 0.025$ & -0.131 & 0.888 & $0.187 \pm 0.028$ \\
$0.4-0.5$ & $0.166 \pm 0.024$ & -0.127 & 0.892 & $0.201 \pm 0.027$ \\
$0.5-0.6$ & $0.171 \pm 0.023$ & -0.142 & 0.898 & $0.207 \pm 0.025$ \\
$0.6-0.7$ & $0.158 \pm 0.023$ & -0.119 & 0.892 & $0.191 \pm 0.026$ \\
\hline
\end{tabular}

\begin{tabular}{|cccc|}
\hline $\cos \theta_{T}$ & $\frac{2 \cos \theta_{T}}{1+\cos \theta_{T}^{2}}$ & $\tilde{A}_{M C}^{b}$ & $\left\langle P_{e}\right\rangle_{\mathcal{L}} \cdot \tilde{A}_{M C}^{b}$ \\
\hline $0.0-0.1$ & 0.010 & $0.041 \pm 0.012$ & $0.030 \pm 0.009$ \\
$0.1-0.2$ & 0.293 & $0.108 \pm 0.012$ & $0.080 \pm 0.009$ \\
$0.2-0.3$ & 0.471 & $0.190 \pm 0.012$ & $0.140 \pm 0.009$ \\
$0.3-0.4$ & 0.624 & $0.249 \pm 0.011$ & $0.184 \pm 0.008$ \\
$0.4-0.5$ & 0.748 & $0.317 \pm 0.011$ & $0.235 \pm 0.008$ \\
$0.5-0.6$ & 0.846 & $0.335 \pm 0.010$ & $0.248 \pm 0.008$ \\
$0.6-0.7$ & 0.914 & $0.352 \pm 0.011$ & $0.260 \pm 0.008$ \\
\hline
\end{tabular}

asymmetry fit which accounts for the analyzing power of the measurement. The other point at which model dependence is introduced is the light-quark subtraction step. A summary of the systematic errors in this analysis is presented at the end of this section in Tables 6.6, 6.7, 6.8, and 6.9.

\subsubsection{Detector Modeling}

\section{Tracking Efficiency}

The tracking efficiency error is estimated by varying the tracking efficiency in the Monte Carlo and estimating its effect on the analyzing power. Because the effect of tracking efficiency decouples from the other simulation issues, and because detailed studies of the tracking performance show that any unmodeled inefficiency has at most a very weak dependence on track $\cos \theta$ and momentum [149], the model has been to remove tracks completely at random from the Monte Carlo. It was found that the sensitivity of the measured value of $A_{b}$ is

$$
\frac{1}{A_{b}} \frac{\partial A_{b}}{\partial \epsilon_{\text {inef }}} \simeq-2.0
$$

where $\epsilon_{\text {ine }}$ is the residual unmodeled overall tracking inefficiency.

This unmodeled inefficiency may be estimated by comparing the data multiplicity distributions with those obtained from the Monte Carlo simulation. Figure 4.4 shows the raw multiplicity distribution for all hadronic events passing event selection criteria. The average multiplicity of selected tracks in data events passing hadronic event selection is 17.10 , while the simulation reports 17.51 . Figure 4.6 shows the distribution in polar angle of tracks in those events compared between data and Monte Carlo, and Figure 4.5 shows a comparison as a function of the natural logarithm of the total momentum.

The difference in the observed multiplicity between data and Monte Carlo can arise from two sources: 1) unmodeled tracking inefficiency, and 2) mis-tuning of the Monte Carlo generator so that it does not produce the genuine $Z^{0}$ decay charged multiplicity. Given a multiplicity measurement from ALEPH of $\left\langle n_{c h}\right\rangle=20.85 \pm 0.24$ [150] and that the SLD Monte Carlo generator produces $\left\langle n_{c h}\right\rangle=20.9$, and an overall mulfiplicity discrepancy between data and Monte Carlo of 0.4 tracks/ event out of 17.1, the unmodeled tracking inefficiency is estimated to be $2.3 \%$. This may be easily corrected for after the fact, either by removing tracks from the Monte Carlo in a random fashion, or by applying an ad hoc correction to the answer after the analysis is complete. The former approach was chosen, and has been incorporated in the answer quoted in the previous section. 
The systematic error incurred in this process is cautiously estimated to be $2 \%$ on the tracking efficiency, which translates into a $4 \%$ relative uncertainty on $A_{b}$.

\section{Ghost Tracks}

A feature of Figure 4.5 is that while the data has fewer tracks than the Monte Carlo for momenta up to $3 \mathrm{GeV}$, it has more tracks at high momentum. It is likely that this corresponds to an error in the modeling of the fragmentation and hadronization processes by JETSET, but the most conservative approach to understanding this as an error is to take all of the extra tracks to be randomly-signed background.

The "ghost track" hypothesis has a conceivable mechanism. High-momentum tracks in the cores of jets may rob hits from each other, and extra tracks may be formed out of the shorter fragments left over after the long tracks have been found. These in general will be quite straight, due to the projective nature of the CDC cells, the high momenta of the original parent tracks, and the probability of misassigning hits to form tracks.

The Monte Carlo has been conservatively adjusted in this analysis to add extra, randomly-signed tracks of the right momentum-distribution to flatten out the ratio of Figure 4.5. The end result of this test is that the measured value of $A_{b}$ changed by a relative $1.0 \%^{* *}$.

\section{Momentum Resolution}

The momentum resolution of the CDC, discussed in Section 4.2.3, is measured using dilepton events and through-going cosmic rays, and is found to be

$$
\delta p_{\perp}=p_{\perp}^{2} \sqrt{0.0050^{2}+\left(0.010 / p_{\perp}\right)^{2}},
$$

where $p_{\perp}$ is the momentum transverse to the beam axis in $\mathrm{GeV}$. The effect of the momentum resolution on the analyzing power of the technique can be estimated from the Monte Carlo by calculating the analyzing power of Equation 6.3 with a generatorlevel Monte Carlo with a simple acceptance model and cuts. For the same events,

\footnotetext{
"* The effect of extra tracks at high momentum increases with larger $\kappa$.
}

the momentum of the tracks is smeared by the amount specified in Equation 6.29, and the analyzing power recomputed. Taking the entire difference as a conservative estimate of the effect yields a relative systematic error of $<0.2 \%$.

\section{Tag Purity}

Section 6.2.5 dealt with issues of the $B$ tag affecting the Monte Carlo estimation of its purity. To estimate the effect of mismodeling the purity, the analysis is re-run, forcing the contamination fraction to be different from what the Monte Carlo predicts by $1 \sigma$ of the error. In the worst case, the all of the impurity is assumed to be $Z^{0} \rightarrow c \bar{c}$ background, because these events have opposite asymmetry to $Z^{0} \rightarrow b \bar{b}$ events. It is found that

$$
\partial A_{b}^{\text {meas }} / \partial \Pi_{c}^{\text {estimated }}=1.48
$$

and so the total systematic error from the tag composition is $2.4 \%$. Contributions from $u d s$ contamination are smaller than the charm contribution by a factor of 10 , and furthermore have the same sign asymmetry as the $B$ asymmetry, and therefore do not contribute significantly.

The $Z^{0} \rightarrow \tau^{+} \tau^{-}$contamination was discussed above, and does not contribute more than $(0.04 \pm 0.02) \%$ of the tagged sample, and therefore its contribution to the asymmetry cannot be more than $0.04 \%$, assuming randomly signed directions. Non$Z^{0}$ events in the detector constitute a smaller fraction of the event sample than do the $Z^{0} \rightarrow \tau^{+} \tau^{-}$, due to the difficulty of satisfying both the event selection and the impact-parameter tag.

A more serious contribution from background tracks arises from the fact that some of these background events may overlay beam crossings on which a real $Z^{0}$ decayed, even ones that are tagged. This is modeled by the background overlay in the Monte Carlo.

\section{Geometrical Distortion of the CDC}

The tracking chamber has been aligned using high-momentum tracks from $Z^{0} \rightarrow$ $\mu^{+} \mu^{-}$and wide-angle Bhabhas. These provide a convenient source of monoenergetic 

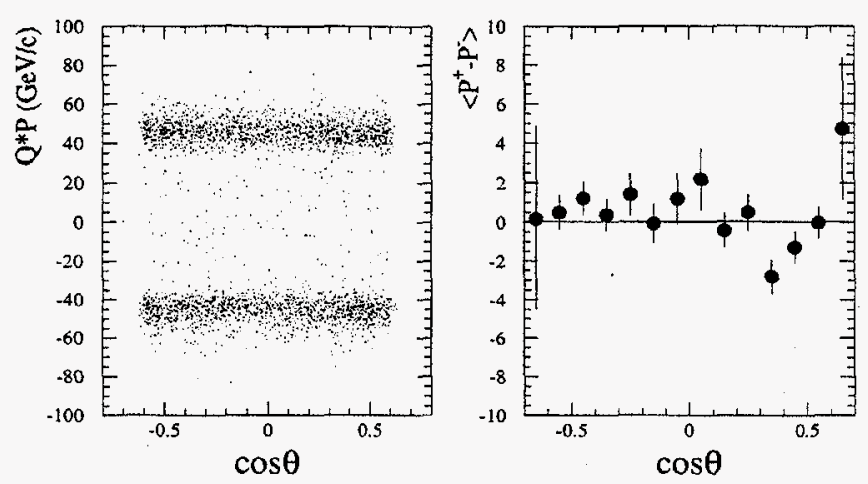

Figure 6.16: Total momenta of positive and negative muons separately as a function of $\cos \theta$. Negative muons appear with negative momenta in the plot on the left. The plot on the right shows the average difference between the measured total momentum of positive muons and negative muons as a function of polar angle.

tracks to test if there are any biases in the momentum of positive and negative tracks in the forward and backward hemispheres. Figure 6.16 shows the momentum of positive and negative leptons separately in di-lepton events as a function of $\cos \theta$. ruling out distortions which would bias the momentum of a $45 \mathrm{GeV}$ track by more than $2 \%$. Distortions produce constant offsets in the sagitta of tracks, and therefore affect lower-momentum tracks less than the dimuon sample.

The left-right forward-backward asymmetry is a particularly convenient observable from the point of view of this systematic error, in that the measured asymmetry is formed as a difference between the polarization states, and therefore, since the tracking chamber has the same geometrical distortions with the left-handed electron beam as with the right-handed beam, the distortions nearly cancel. The residual distortion effect is proportional to $A_{\varepsilon} \simeq 0.15$, owing to the fact that more events are produced with the left-handed beam. This particular distortion is a $\sim 6$ times more serious problem for the unpolarized forward-backward asymmetry.
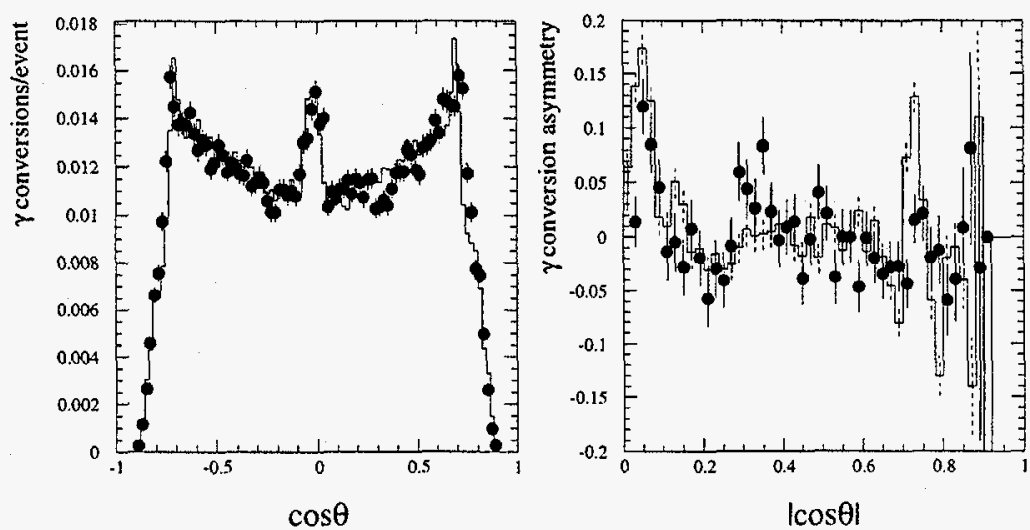

Figure 6.17: Data and Monte Carlo distributions of gamma conversions and their asymmetries.

\section{Detector Material Asymmetry}

Nuclear interactions of final-state particles with the material of the detector before the tracking volume introduce extra tracks into the detector, as well as degrading the momentum of the particles originating with the $Z^{0}$ decay. The positive charge of atomic nuclei lends the the extra tracks a net positive bias, and gives them a larger baryon fraction than is present in tracks originating at the $Z^{0}$ decay vertex. The extra tracks produced tend to have low momentum and are thus given lower weight in this analysis.

For the same reason that the geometrical distortion errors are suppressed by a factor of $A_{e}$, errors induced by asymmetry in the detector material distribution are also suppressed by the same amount.

Detector material asymmetry can be estimated by examining the rate of gamma conversions as a function of polar angle. Shown in Figure 6.17 is the gamma conversion rate on hadronic $Z^{0}$ decays passing the event selection compared between data and 
Monte Carlo for the 1994-1995 run. The asymmetry

$$
A_{\gamma}=\frac{n_{\gamma}(\cos \theta)-n_{\gamma}(-\cos \theta)}{n_{\gamma}(\cos \theta)+n_{\gamma}(-\cos \theta)}
$$

is also computed with its statistical error and compared against the Monte Carlo. The significant rise in asymmetry around $\cos \theta \sim 0.05$ owes itself to the presence of a steel screw and a steel band around the VXD cryostat at the physical midplane of the detector. Because the IP is $\sim 3.9 \mathrm{~mm}$ offset from the geometrical center of the detector, this band and screw are offset to one side. Fortunately, this material imbalance is in the central portion of the detector and contributes a vanishingly small amount to the asymmetry.

In order to quantify the detector material asymmetry's effect on an asymmetry measurement, a properly weighted average of it is necessary. Events at larger values of $|\cos \theta|$ contribute more to the fit, as per Equation 1.72. The statistical errors are magnified to reflect their contribution in the fit,

$$
\delta A_{\gamma} \rightarrow \delta A_{\gamma}\left(\frac{1+\cos ^{2} \theta}{2 \cos \theta}\right)
$$

and the asymmetries $A_{\gamma}$ are averaged with their new errors within the range $|\cos \theta|<$ 0.8 , the tracking acceptance cut. The averages found are $\left\langle A_{\gamma}\right\rangle=-0.62 \%$ for the data and $\left\langle A_{\gamma}\right\rangle=-0.91 \%$ for the Monte Carlo. The discrepancy between these, multiplied by $A_{e}$, gives the justification for quoting this error at $<0.1 \%$.

\section{Thrust Axis Resolution}

The tracking chamber can be used to estimate the reliability of the Monte Carlo simulation of the thrust axis resolution. The angular resolution of the tracks in the CDC is $0.45 \mathrm{mrad}$ in $\phi$ and $3.7 \mathrm{mrad}$ in $\theta$ [151], much more precise than the thrust axis needs to be known. The technique is to sample events with calorimetric thrust axes in a particular bin of $\cos \theta$ and then to histogram the $\cos \theta$ of tracks in all such events. To the extent that JETSET properly models the angular distribution of tracks in $Z^{0}$ decays, these distributions of track angles can be compared directly between data and Monte Carlo.
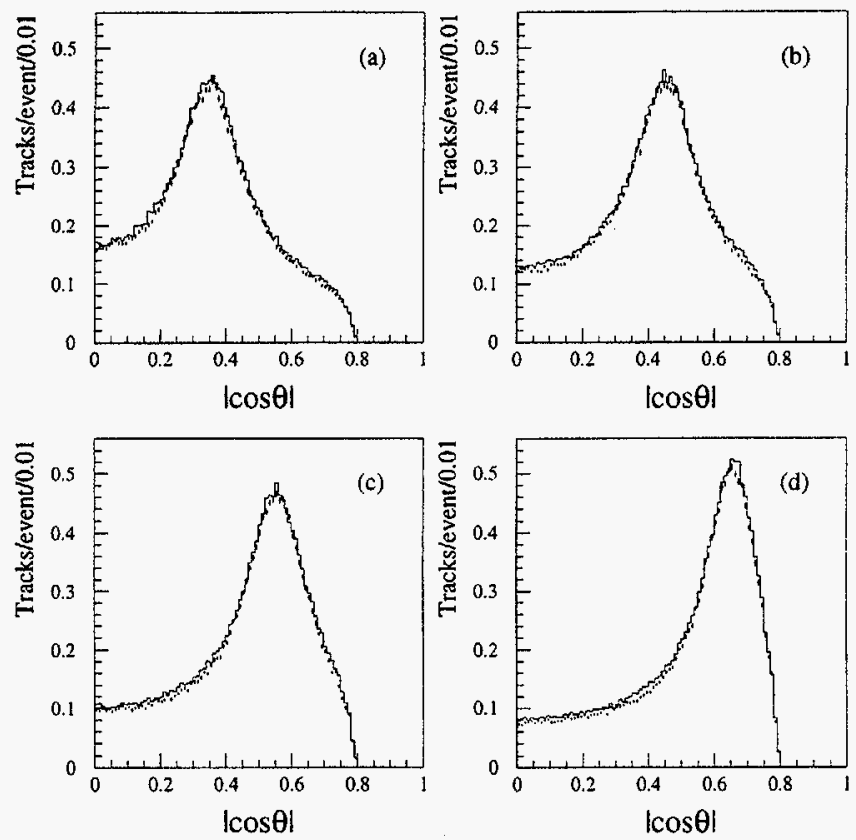

Figure 6.18: Comparison of data (points) and Monte Carlo (histogram) track $\cos \theta$, in bins of calorimeter thrust $\cos \theta_{T}$, showing that the calorimeter thrust axis direction is well modeled in the Monte Carlo. The $\cos \theta_{T}$ bins are: (a) $0.3<\left|\cos \theta_{T}\right|<.4$, (b) $0.4<\left|\cos \theta_{T}\right|<.5$, (c) $0.5<\left|\cos \theta_{T}\right|<.6$, and (d) $0.6<\left|\cos \theta_{T}\right|<.7$. 
If the data has an unmodeled smearing of the thrust axis, or if the Monte Carlo smears the axis too much, the effect of that will appear in these distributions. The smearing must be sufficient to move an event from one region in $\cos \theta$ to another with a significantly different expected value of asymmetry as predicted by Equation 1.72. A comparison of these distributions, shown in Figure 6.18, puts an upper bound on the thrust axis bias at $\delta(\cos \theta)=0.005$, which would bias the $A_{b}$ answer by $0.6 \%$ relative to itself.

The momentum-weighted track charge in Equation 6.2 is insensitive to small angular smears of the thrust axis. Changes to the dot product of the momentum into the thrust axis are second order in the smearing angle. The effect on the transverse momentum of particles with respect to the thrust axis is much larger, although not important for this analysis.

\section{Event Pileup}

A small, known deficiency of the Monte Carlo is that it only generates one hadronic $Z^{0}$ decay per event, while each beam crossing at SLC has a finite probability of producing two $Z^{0}$ decays on one beam crossing. Because SLC has a higher luminosity than LEP per beam crossing, it has a larger fraction of pileup events. Assuming 150,000 hadronic $Z^{0}$ decays are collected at an average running luminosity of 50 decays/hour with SLC running at $120 \mathrm{~Hz}$, the pileup fraction should be $\sim 1 / 8640$, yielding a total of 17.3 pileup events. After correcting for event selection efficiency and the tagging fraction, $\sim 4.7$ events are expected to be pileup events. Of a total tagged sample of 12002 events, the pileup fraction amounts to $0.04 \%$. If the pileup events are assumed to be randomly signed, then the systematic error on $A_{b}$ is also $0.04 \%$. In order to tag, though, a pileup trigger has to contain at least one $Z^{0} \rightarrow b \bar{b}$, so the effect is very likely smaller.

\section{Final-State $\mathrm{QCD}$ radiation}

The asymmetry-diluting effects of final-state QCD radiation are incorporated into the JETSET Monte Carlo through its parton-shower model. The magnitude of the difference between the raw asymmetry and the QCD corrected asymmetry can be

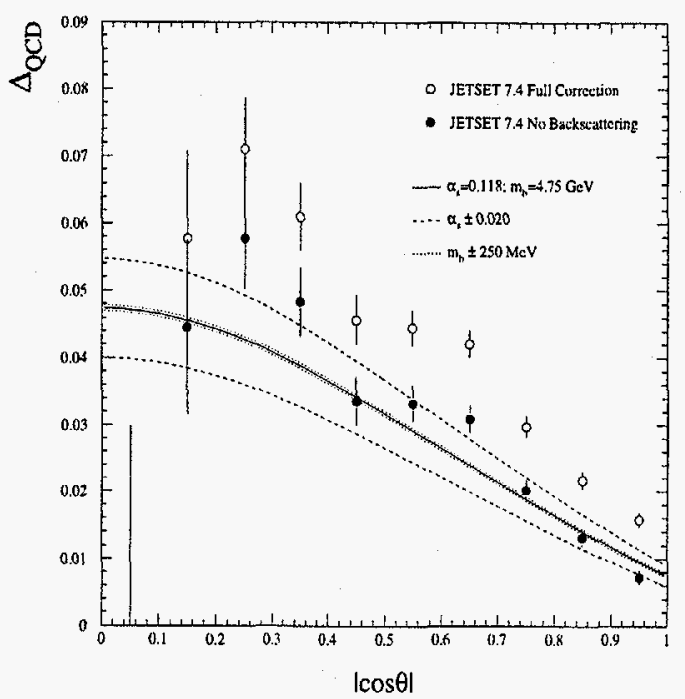

Figure 6.19: QCD corrections as calculated in [58], compared against those estimated from JETSET 7.4. Errors on the curves include $\pm 2 \sigma$ of the theoretical error of $\alpha_{s}$ and $\pm 250 \mathrm{MeV}$ on the $b$ quark mass. The open circles are the full correction as estimated by the JETSET generator, and the filled circles are the correction omitting events in which the two quarks are in the same hemisphere. This second form will become important in Section 7.3.6. The difference between JETSET and the analytic calculation gives a $-1 \%$ relative correction to $A_{b}$.

calculated using JETSET by comparing the asymmetry using the quark axis before the initiation of the parton shower and the asymmetry using the quark axis after the parton shower has reached its cutoff. The former asymmetry is simply the raw electroweak value given in Equation 1.72 as a function of $\cos \theta$. This smear in the quark axis angle dilutes the asymmetry and constitutes a high-order calculation of the QCD correction, which may be compared against first- and second-order analytic calculations. This comparison of the fractional change in asymmetry using the Monte Carlo estimate and the calculation in Reference [58] is shown in Figure 6.19. 
Table 6.3: Systematic uncertainties on the electron beam polarization as measured by the Compton Polarimeter [42]. The later 2/3 of the 1993 running benefited from routine laser polarization scanning and has smaller errors.

\begin{tabular}{|lccc|}
\hline & End 1993 & Total 1993 & $1994-1995$ \\
Systematic Uncertainty & $\delta P_{e} / P_{e}(\%)$ & $\delta P_{e} / P_{e}(\%)$ & $\delta P_{e} / P_{e}(\%)$ \\
\hline Laser Polarization & 0.6 & 1.0 & 0.2 \\
Detector Linearity & 0.6 & 0.6 & 0.5 \\
Electronic Crosstalk & 0.2 & 0.2 & 0.2 \\
Analyzing Power Calibration & 0.4 & 0.4 & 0.3 \\
Cross Checks & 0.5 & 0.5 & 0.4 \\
\hline Polarimeter Total & 1.1 & 1.3 & 0.76 \\
\hline Final Focus Chromaticity & 1.1 & 1.1 & 0.2 \\
\hline Total & 1.6 & 1.7 & 0.78 \\
\hline
\end{tabular}

It is apparent from Figure 6.19 that JETSET estimates a larger QCD correction than the analytic calculation. Because the analytic version is used in Chapter $\tau$, consistency requires a commensurate $\Delta_{Q C D}$ to be used in this result. The values of the estimate Monte Carlo asymmetry in Table 6.2 have been increased by a relative $1 \%$, which lowers the measured value of $A_{b}$ by $1 \%$.

The systematic error estimated from this contribution is given by allowing the value of $\alpha_{s}$ to vary by $2 \sigma$ of its current theoretical ambiguity, as well as comparing a second-order calculation [54] against the first-order version [55]. The second-order calculation was not used owing to the fact that it neglected the $b$ quark mass, did. not give a dependence on $\cos \theta$, and was calculated assuming the $b$ quark direction was-measured, instead of the thrust axis. It has been estimated that using the thrust axis instead of the $b$ quark axis reduces the QCD correction to $90 \%$ of its value [152]. but another calculation [57] quotes a $95 \%$ correction, and this discrepancy is also accounted in the systematic error.

\section{Polarization}

The systematic error due to the measurement error of the electron beam polarization is one of the smallest errors due to the effort involved in producing the required precision for the $A_{L R}$ measurement. The Compton polarimeter measures a raw scattering asymmetry, which is then divided by the laser polarization and an expected asymmetry function which depends on a QED prediction and a detector model. The raw Compton asymmetry is affected by detector/ADC linearity and interchannel crosstalk. The raw asymmetry also involves subtracting the laser-off background from the observed Compton signal. If there is crosstalk from the laser electronics over to the detector amplifiers and digitizers which contributes to the laser-on signal but not to the measured background, it will affect the measured asymmetry. Fortunately, this electrical pickup can be measured when the SLC electron beam is off.

The laser polarization is monitored by scanning the voltages on the Pockels Cells to determine the phase shift of the transport optics down to the Compton interaction point, and to determine the unpolarized fraction, which dominates the uncertainty in laser polarization. This scanning of Pockels Cell voltages was not installed until about $1 / 3$ of the way through the 1993 run, increasing the laser polarization error for the 1993 result.

An additional error in the polarization used arises from the fact that the Compton polarimeter measures the average polarization of the entire bunch of electrons, while only a portion of the bunch is focused tightly enough to contribute to the luminosity: If the electron bunch has a large energy spread, or worse, a long energy tail, then the off-energy electrons in the bunch will not arrive at the SLC IP properly focused, owing to the chromaticity of the final focus. The energy of the electrons is correlated with the orientation of their polarization, from Equation 3.2. Energy spread in the bunch depolarizes the bunch, but a chromatic final focus will pick out a more coherently polarized portion of the bunch. This effect has been estimated for 1993 running parameters to be a $(1.7 \pm 1.1) \%$ correction to the luminosity-weighted polarization [ 41 ]. For 1994-1995 running, the energy spread of the beam was carefully controlled by "overcompressing" the bunch in the NRTL transfer line, and by upgrading the optics of the final focus. For 1994-1995, the chromatic effect is estimated to be $0.2 \pm 0.2 \%$. and it is incorporated in both the polarization values and errors for both runs. 


\section{Beam Parameter Asymmetries}

A potential source of error for an asymmetry measurement is the difference in the average absolute value of the polarization on the left-handed beam pulses as compared to the right-handed beam pulses. If we follow Equation 1.61 but set $P_{e_{1} \text { Right }}=|P|+\epsilon_{p}$, and $P_{e, L e f t}=-|P|+\epsilon_{p}$, where $|P|$ is the average magnitude of the polarization of both beams, then the left-right forward-backward asymmetry of Equation 1.72 becomes

$$
\tilde{A}=\frac{2 A_{b}|P| \cos \theta}{\left(1+\cos ^{2} \theta\right)\left(1-A_{e} \epsilon_{p}\right)} .
$$

The Compton polarimeter is able to measure the polarization of both helicity states of the beam separately, and is measured to be $\Delta P_{e} / P_{e}=0.0033 \pm 0.0001$ [153] and is smaller in 1994-1995 [42]. The effect on the measured $A_{b}$ is a relative $0.05 \%$.

A related systematic error contribution is the difference in average luminosity between the left-handed beam and the right-handed beam ${ }^{\dagger \dagger}$. This asymmetry can be measured with the electron beam toroids and corrected after the run. During the 1993 run, the NLTR spin rotator solenoid was reversed for approximately half of the accumulated luminosity, nearly canceling this asymmetry; The net current asymmetry for 1993 was estimated to be $(0.038 \pm 0.050) \%$ [153]. For 1994-1995, the spin rotator reversals were arranged less fortuitously, and the average luminosity asymmetry became $(0.087 \pm 0.043) \%$ [42]. If $\mathcal{L}_{R}=\bar{L}\left(1+\epsilon_{\mathcal{L}}\right)$ and $\mathcal{L}_{L}=\bar{L}\left(1-\epsilon_{\mathcal{L}}\right)$, the expected asymmetry function becomes

$$
\tilde{A}=\frac{2 A_{b}\left|P_{e}\right| \cos \theta}{\left(1+\cos ^{2} \theta\right)\left(1-\left(1+\left|P_{e}\right|\right) A_{e} \epsilon_{\mathcal{L}}\right)} .
$$

This effect, too, is multiplied by a factor of $A_{\varepsilon}$, but has an additional $\left(1+\left|P_{\varepsilon}\right|\right)$. The total error incurred from neglecting this effect is $0.03 \%$.

ThWhile the polarized SLC is constructed to cancel most effects that could generate such an asymmetry, effects at the polarized electron source can remain. In particular, misalignment or circular dichroism in the source optics can introduce a difference in the amount of light reaching the cathode for the two photon helicities.

\subsubsection{Modeling Physical Processes}

\section{Weighting Scheme}

The task of evaluating the sensitivity of an analysis that is sensitive to the details of $B$ hadron fragmentation and decay using a Monte Carlo model is a large one. The technique used here is to generate a large sample of JETSET $7.4 Z^{0} \rightarrow b \bar{b}$ decays and to calculate the analyzing power. No detector simulation is performed, because systematic effects from the underlying physics model and detector-induced effects are expected to be independent multiplicative factors in the analysis.

The strategy employed is to evaluate the decay axis from the original quark directions given by JETSET 7.4, calculate the momentum-weighted charge sum for the event using observable final-state particles (after decays of particles, including the $K_{s}^{0}$ and the $\Lambda$. Then, to calculate the error coming from contribution $j$, the sums of correct and incorrect events, with weights to be described below, are formed:

$$
N_{\text {right }}^{j}=\sum_{\text {CorrectEvents }}\left(1+\epsilon_{i}^{j}\right)
$$

where $i$ is the subscript of correct events, and $\epsilon_{i}^{j}$ is the weight on that event for systematic error $j$. Similarly, the incorrect sum is

$$
N_{\text {wrong }}^{j}=\sum_{\text {InorrectEvents }}\left(1+\epsilon_{i}^{j}\right) \text {. }
$$

The weighted analyzing power is given by

$$
A P^{j}=\frac{N_{\text {right }}^{j}-N_{w r o n g}^{j}}{N_{\text {right }}^{j}+N_{w r o n g}^{j}},
$$

and compared against the analyzing power computed for the same generated sample with $\epsilon=0$ on each event. The fractional change in analyzing power is the fractional error on the measurement of $A_{b}$, corresponding to a fluctuation in a model paraineter described by the weights $\epsilon_{i}^{j}$. There is no need to normalize the weights in this scheme because of the the cancelation of normalization factors in the numerator and denominator of Equation 6.37. Some of the errors mentioned below will use special estimations not incorporating the weighting scheme. 
Table 6.4: Generator-level analyzing powers for events with different numbers of fragmentation protons. The errors are statistical errors on this Monte Carlo study.

\begin{tabular}{|ccc|}
\hline \# Fragmentation Protons & Event Fraction & Analyzing Power \\
\hline 0 & $0.534 \pm 0.001$ & $0.4767 \pm 0.0027$ \\
1 & $0.237 \pm 0.001$ & $0.4339 \pm 0.0041$ \\
2 & $0.159 \pm 0.001$ & $0.4371 \pm 0.00050$ \\
3 & $0.046 \pm 0.0005$ & $0.4037 \pm 0.0095$ \\
4 & $0.0178 \pm 0.0003$ & $0.414 \pm 0.015$ \\
5 & $0.0039 \pm 0.0001$ & $0.374 \pm 0.033$ \\
$\geq 6$ & $0.0014 \pm 0.0001$ & $0.465 \pm 0.054$ \\
\hline
\end{tabular}

\section{$B$ Fragmentation and Hadronization}

The first systematic error to test is the fragmentation function, which will use special Monte Carlo generations, as the fragmentation function affects the momentum and distribution of all particles in the event. If a choice were made to weight the events with a function of the $B$ hadron momentum, then the sample may be biased towards events with hard gluon radiation, rather than the details of string breaking, so the weighting procedure was not chosen.

The analyzing power of the momentum-weighted track charge technique was calculated with the Peterson fragmentation function [118] with $\epsilon_{b}=0.006$, yielding an average $x_{e}=E(B) / E($ beam $)=0.695$. The Peterson parameter $\epsilon_{b}$ was varied between 0.01 and 0.002 to estimate this systematic error. Also investigated was the use of the Bowler fragmentation function [154], tuned to have the same mean $B$ hadron energy as the default Peterson function, and the discrepancy in analyzing power is given as a separate error

The uncertainty in the production fractions of $B$ hadron species contributes a systematic error because the different species have different analyzing powers. The $B^{0}$ and $B^{+}$fractions are constrained to be equal by isospin symmetry, but the $B_{s}$ and $\Lambda_{b}$ fractions need to be measured experimentally [155][156][157].
Table 6.5: Generator-level analyzing powers for events with different numbers of fragmentation $K^{ \pm}$. The errors are statistical errors on this Monte Carlo study.

\begin{tabular}{|ccc|}
\hline \# Fragmentation $K^{ \pm}$ & Event Fraction & Analyzing Power \\
\hline 0 & $0.382 \pm 0.001$ & $0.4873 \pm 0.0032$ \\
1 & $0.277 \pm 0.001$ & $0.4541 \pm 0.0038$ \\
2 & $0.189 \pm 0.001$ & $0.4444 \pm 0.00046$ \\
3 & $0.0884 \pm 0.0006$ & $0.4072 \pm 0.0069$ \\
4 & $0.0399 \pm 0.0004$ & $0.3942 \pm 0.010$ \\
5 & $0.0148 \pm 0.0003$ & $0.3848 \pm 0.016$ \\
$\geq 6$ & $0.0086 \pm 0.0002$ & $0.3617 \pm 0.023$ \\
\hline
\end{tabular}

Another detail of fragmentation is the fraction of protons and other baryons produced by the hadronization process. Because protons are much more massive than the other light particles which contribute to the momentum-weighted charge sum, they can substantially affect the analyzing power. A Monte Carlo run consisting of only $Z^{0} \rightarrow b \bar{b}$ was performed, and the analyzing power computed separately for events with different numbers of fragmentation protons present. The results of this study are presented in Table 6.4. The SLD Monte Carlo generates 1.053 protons/event [36], while the PDG value is $0.92 \pm 0.11$ [146]. This does not constrain the proton production in $Z^{0} \rightarrow b \bar{b}$ events, though, which the Monte Carlo predicts at 0.78 protons/event, and so the uncertainty is doubled to be conservative. If the sample of events with fragmentation protons was reduced by $20 \%$ (absolute) and replaced with events with no fragmentation protons in them, the analyzing power would change by a relative $2.4 \%$

The multiplicity of fragmentation $K^{ \pm}$was treated similarly, and this result is displayed in Table 6.5. The 1994 PDG reports an inclusive $2.12 \pm 0.13 K^{ \pm}$event, while the SLD Monte Carlo predicts 2.28 inclusive kaons in all hadronic $Z^{0}$ decays, including the decay products of heavy hadrons. The SLD Monte Carlo predicts 1.2 fragmentation kaons per event in $Z^{0} \rightarrow b \bar{b}$ decays. If a conservative $20 \%$ of all events were replaced with events without fragmentation kaons, then the analyzing power 
Table 6.6: Summary of the detector modeling and general systematics of the binned asymmetry fit to $A_{b}$.

\begin{tabular}{|c|c|c|}
\hline Error Source & Variation & $\delta A_{b} / A_{b}$ \\
\hline \multicolumn{3}{|l|}{ Tracking } \\
\hline Tracking efficiency & $2.0 \%$ & $4.0 \%$ \\
\hline Ghost Tracks & $3 \%$ at high momentum & $1.0 \%$ \\
\hline Momentum Resolution & $100 \%$ of effect & $<0.2 \%$ \\
\hline Thrust Axis Resolution & & $0.6 \%$ \\
\hline Chamber Distortion & & $<0.3 \%$ \\
\hline Material Asymmetry & & $<0.1 \%$ \\
\hline \multicolumn{3}{|l|}{ Tag Contamination } \\
\hline$Z^{0} \rightarrow c \bar{c}$ Contribution to Tag & See Table 6.1 & $2.6 \%$ \\
\hline$Z^{0} \rightarrow u d s$ Contribution to Tag & See Table 6.1 & $0.3 \%$ \\
\hline$Z^{0} \rightarrow \tau^{+} \tau^{-}$Contribution to Tag & Full Value & $<0.04 \%$ \\
\hline \multicolumn{3}{|l|}{ General } \\
\hline Monte Carlo Statistics & $1 \sigma$ & $1.6 \%$ \\
\hline QCD Radiation & $\alpha_{s} \pm 0.02,2^{\text {nd }}$ order terms & $0.9 \%$ \\
\hline Polarization & $1 \sigma$ & $0.8 \%$ \\
\hline Event Pileup & $(0.0 \pm 0.26) \%$ & $<0.04 \%$ \\
\hline Subtotal: Detector Modeling and & General & $5.3 \%$ \\
\hline
\end{tabular}

Table 6.7: Summary of the $B$ hadron fragmentation systematic errors of the binned asymmetry fit to $A_{b}$.

\begin{tabular}{|lcc|}
\hline Error Source & Variation & $\delta A_{b} / A_{b}$ \\
\hline$B$ Fragmentation $\left\langle x_{B}\right\rangle$ & $\left\langle x_{B}\right\rangle=0.695 \pm 0.021$ & $1.3 \%$ \\
$B$ Fragmentation Function & Bowler-Peterson, same $\left\langle x_{e}\right\rangle$ & $0.5 \%$ \\
Fragmentation Protons & $(0.78 \pm 0.16) \%$ & $2.4 \%$ \\
Fragmentation $K^{ \pm}$ & $(1.20 \pm 0.24) \%$ & $2.5 \%$ \\
$b \rightarrow B^{0}$ Fraction & $(40.6 \pm 2.0) \%$ & $0.39 \%$ \\
$b \rightarrow B^{+}$Fraction & $(40.6 \pm 2.0) \%$ & $0.80 \%$ \\
$b \rightarrow B_{s}$ Fraction & $(11.5 \pm 2.0) \%$ & $0.63 \%$ \\
$b \rightarrow \Lambda_{b}$ Fraction & $(7.3 \pm 3.6) \%$ & $0.58 \%$ \\
gluon $\rightarrow b \bar{b}$ & $\pm 50 \%$ of JETSET & $0.02 \%$ \\
gluon $\rightarrow c \bar{c}$ & $\pm 50 \%$ of JETSET & $0.07 \%$ \\
\hline Subtotal: $B$ Fragmentation & & $3.9 \%$ \\
\hline
\end{tabular}

would change by a relative $2.5 \%$.

The inclusive pion yield in the SLD Monte Carlo matches the 1994 PDG value to within $0.4 \%$, and the error in the PDG average is $2 \%$, so fragmentation pions are not expected to be a significant source of systematic error. The inclusive non-leading multiplicity has also been measured by SLD [158], and found to be in agreement with the SLD Monte Carlo model.

\section{$B^{0}-\bar{B}^{0}$ Mixing}

The-systematic error from uncertainties in the species-averaged time-integrated mixing parameter $\chi_{B}$ have been evaluated by disabling the mixing in the Monte Carlo, and checking the difference in analyzing power between that model and the Monte Carlo with a full mixing value of $\chi_{B}=0.130$ applied. The total effect on the analyzing power is a relative $11 \%$, and the fractional uncertainty in $\chi_{B}$ is proportional to the 
Table 6.8: Summary of the $B$ hadron mixing and decay systematic errors of the binned asymmetry fit to $A_{b}$.

\begin{tabular}{|lcc|}
\hline Error Source & Variation & $\delta A_{b} / A_{b}$ \\
\hline$B$ Mixing & $\chi_{B}=0.115 \pm 0.006$ & $0.6 \%$ \\
$\tau_{B^{+}} / \tau_{B^{0}}$ Lifetime Ratio & $1.00 \pm 0.20$ & $0.22 \%$ \\
$D^{0}$ fraction in $B$ decay & $(64.8 \pm 2.6) \%$ & $0.24 \%$ \\
$D^{ \pm}$fraction in $B$ decay & $(26.6 \pm 3.7) \%$ & $0.20 \%$ \\
$D_{s}$ fraction in $B$ decay & $(10.7 \pm 2.5) \%$ & $0.9 \%$ \\
$B \rightarrow D$ Momentum & $\left\langle P_{D}\right\rangle=(2.50 \pm 0.13) \mathrm{GeV} / \mathrm{c}$ & $0.10 \%$ \\
$B^{+} \rightarrow D$ vs $B^{0} \rightarrow D$ Momentum & $\pm 20 \%$ & $3.7 \%$ \\
$B$ Decay Multiplicity & $11.04 \pm 0.25$ & $0.41 \%$ \\
$B \rightarrow K^{ \pm}$Multiplicity & $1.49 \pm .06$ & $0.22 \%$ \\
$B \rightarrow P$ Multiplicity & $0.13 \pm .02$ & $0.09 \%$ \\
$B \rightarrow \pi^{ \pm}$Multiplicity & $8.40 \pm 0.25$ & $0.32 \%$ \\
$B \rightarrow K^{ \pm}$Momentum & $4 \times$ hard tail & $0.62 \%$ \\
$B \rightarrow P$ Momentum & $4 \times$ hard tail & $0.10 \%$ \\
$B \rightarrow \pi^{ \pm}$Momentum & $4 \times$ hard tail & $3.0 \%$ \\
$B \rightarrow l \nu X$ B.R. Fraction & $10.96 \pm 0.55$ & $0.46 \%$ \\
$B \rightarrow D^{*}$ Fraction & $(62 \pm 12) \%$ & $0.32 \%$ \\
$\Lambda_{b}$ Polarization & $100 \%$ of expectation & $0.8 \%$ \\
\hline$S u b t o t a l: B$ Mixing and Decay & & $5.1 \%$ \\
\hline
\end{tabular}

Table 6.9: Summary of the charmed hadron modeling systematic errors of the binned asymmetry fit to $A_{b}$.

\begin{tabular}{|lcc|}
\hline Error Source & Variation & $\delta A_{b} / A_{b}$ \\
\hline Charm Fragmentation & & \\
\hline Fragmentation $\left\langle x_{D}^{*}\right\rangle$ & $\left\langle x_{D}^{*}\right\rangle=0.498 \pm 0.023$ & $0.4 \%$ \\
$c \rightarrow D^{0}$ Fraction & $(53 \pm 3) \%$ & $0.07 \%$ \\
$c \rightarrow D^{ \pm}$Fraction & $(25 \pm 5) \%$ & $0.15 \%$ \\
$c \rightarrow D_{s}$ Fraction & $(22 \pm 6) \%$ & $0.11 \%$ \\
\hline Charmed Hadron Decay & & \\
\hline Charm Decay Multiplicity & each $\pm 2.2 \%$ & $0.06 \%$ \\
$D \rightarrow l \nu X$ B.R. Fraction & each $\pm 5 \%(\mathrm{rel})$. & $<.1 \%$ \\
\hline Subtotal: Charm Modeling & & $0.46 \%$ \\
\hline
\end{tabular}

fractional uncertainty in $A_{b}$, supplying the systematic error. A correction must be applied because the latest measurement of the mixing parameter $\chi_{B}=0.115 \pm 0.006$ [61], rather than the older value used in the Monte Carlo. This amounts to a downwards correction to $A_{b}$ of $\simeq 1 \%$.

An important feature is that the mixing dependence of $A_{b}$ when measured with momentum-weighted track charge is less than similar analyses using semileptonic $B$ decays and/or kaons. The reason for this is that the $B^{0}$ and the $B_{s}$ have intrinsically smaller analyzing powers to begin with, so the dilution of the information coming from their decays matters less in the overall sum.

\section{$B$ Meson Decay Model}

The most important sources of model dependence due to the particular choice of the $B$ hadron decay model are described below. Allowed variations in the model parameters are guided by discrepancies between the SLD Monte Carlo and observed data, as mentioned in Chapter 5 , and also by the uncertainties in the available measurements. 


\section{Differences in the $B^{ \pm}$and $B^{0} D$ Spectrum}

The inclusive particle spectra of Figure 5.2 provide no information about the charge of the particles, nor about whether they came from $B^{ \pm}$or $B^{0}$ decay. The $D$ spectra of Figure 5.3 also have no indication of what the individual spectra of $B^{ \pm}$and $B^{0} D$ momentum spectra might be. An error associated with this uncertainty was evaluated by introducing a weight to the $B^{ \pm} \rightarrow D$ momentum spectrum and an opposite weight the $B^{0} \rightarrow D$ spectrum, so that the overall mean would be conserved. The weights are chosen in inverse proportion to the branching fractions, so as to keep the mean $D$ momentum constant.

\section{Hadronic Decays}

One of the main discrepancies between the SLD Monte Carlo and the ARGUS data is in the high-momentum end of the inclusive pion spectrum. We weight events with $\epsilon=1$ for events with $B$-decay pions above $2.0 \mathrm{GeV}$ and report the relative difference in analyzing power as a systematic error. Other errors include varying the average $B$ decay multiplicity, the charm momentum spectrum, the fractions of $D^{0}, D^{+}$, and $D_{s}$ in $B$ hadron decay, as well as the charm decay multiplicity. Errors were also evaluated for the inclusive fractions of kaons, pions, and protons from $B$ decay, as well as their momentum spectra, although these errors may overcount somewhat the charm fractions and spectra errors.

\section{Semileptonic Decays}

While semileptonic decays constitute only $\sim 11 \%$ of the decays of $B$ hadrons, they are an important portion of the sample. The charged lepton has a hard momentum spectrum in the laboratory frame, and it is very correlated with the charge of the $b$ quark emitted by the $Z^{0} ¥$. Leptons from cascade charm decays are anticorrelated with the $b$ sign, but have less momentum than the prompt $B$ decay leptons.

\footnotetext{
${ }^{\ddagger}$ It is not perfectly correlated, because of $B^{0}-\bar{B}^{0}$ mixing.
}

\section{Charm Modeling}

The lifetimes, decay properties, and production momentum spectra of $D$ mesons have already been included in the estimation of the error on the purity of the $B$ tag, but these uncertainties also affect the estimation of how often the correct sign of the decay axis is determined by the momentum-weighted charge in $Z^{0} \rightarrow c \bar{c}$ events. These errors include uncertainties in the species fractions, their multiplicities, and their momentum spectra. Uncertainties in their lifetimes do not enter here, as they are decoupled from the momentum-weighted charge measurements, except to the extent that they alter the composition of the tagged sample.

\section{$\tau_{B^{+}} / \tau_{B^{0}}$ Lifetime Ratio}

A difference in the $B^{+}$and $B^{0}$ lifetimes would have an effect on the analyzing power of the technique, owing to the different analyzing powers of hemispheres containing $B^{+}$and $B^{0}$, and the fact that the tag preferentially selects events containing longlived $B$ hadrons. The magnitude of this effect is diluted somewhat by the fact that there are two $B$ hadrons in each event, each with its chance of contributing enough tracks to tag the event. The SLD Monte Carlo sets the $B^{0}$ and $B^{+}$lifetimes equal to each other, consistent with the latest measurements of $\tau_{B^{+}} / \tau_{B^{0}}$ from LEP and the Tevatron [159] of $1.01 \pm 0.05$.

To investigate this, Monte Carlo events are generated with an artificially modified $\tau_{B^{+}} / \tau_{B^{0}}=1.2$, and the tag and geometrical acceptance are simulated at the generator level. The analyzing power is then computed and compared against generator-level events with equal lifetimes, and the difference between the ratio of the analyzing powers and unity, scaled by the true uncertainty in the lifetime ratio, constitutes the systematic error.

\section{Final-State Polarization}

The average final-state polarization of $b$ quarks emerging from the hard scattering process is $A_{b}$, which in the Standard Model is expected to be close to $94 \%$. If this polarization is transmitted to the final-state hadrons, it has the ability to shift the 
momentum-weighted charge distributions and affect the analyzing power of the technique presented in this chapter.

A recent paper by Falk and Peskin [160] discusses the preservation of polarization of final-state hadrons from $Z^{0}$ decay. In it, they prove a "no-win" theorem, in which it is shown that for the case of $b \rightarrow$ pseudoscalar meson, polarization information is lost. The meson itself is unpolarizable, but the argument of Falk and Peskin shows that even the polarization of a $B^{*}$ cannot affect the final-state distribution of particles, because the angular distribution of the transition photon emitted in $B^{*} \rightarrow B$ has an isotropic distribution. Even transition pions from more highly excited mesons, $B_{1}, B_{2}^{*}$ are expected to have no forward-backwards asymmetry along the direction of helicity of the excited mesons.

Heavy baryons, on the other hand, have the ability to retain their polarization. Because the light diquark in a $\Lambda_{b}$ is in a $j=0$ state, it has no angular momentum to impart to the heavy quark. This argument has been used to show that fragmentation does not dilute $\Lambda_{b}$ polarization when the $\Lambda_{b}$ is produced directly [161][162]. Falk and Peskin, however, add in $30 \% \Sigma_{b}$ and $\Sigma_{b}^{*}$, which have less expected polarization, and arrive at a net polarization of the $\Lambda_{b}$ to be $68 \%$. A recent measurement from ALEPH quotes the $\Lambda_{b}$ polarization, measured with the energies of the lepton and neutrino in semileptonic $\Lambda_{b}$ decays, to be $-0.26 \pm 0.26$ [164], consistent with the high fraction ( $\sim 50 \%$ of the $b$ baryon production) of $\Sigma_{b}$ and $\Sigma_{b}^{*}$ production measured by DELPHI [140].

The polarization of the $\Lambda_{b}$ has an effect on the present analysis because polarized $\Lambda_{b}$ 's are expected to have a forward-backward asymmetry of $\Lambda$ 's as decay products in their rest frame. Körner and Krämer have also calculated a final-state asymmetry in semileptonic decays of polarized $\Lambda_{b}$ 's [163][161][162]. It is expected that the manifestation of polarization in a general hadronic decay of the $\Lambda_{b}$ will be diluted even more than in the semileptonic case.

The expected $\operatorname{BR}\left(b \rightarrow \Lambda_{b}\right)$ is $\sim 7 \%$ [156][157], and so $\sim 14 \%$ of $Z^{0} \rightarrow b \bar{b}$ decays have a $\Lambda_{b}$ in them. The average momentum-weighted charge for the $\Lambda_{b}$ is estimated to be one-half that of the average for the $B$ mesons. Given that an event with a $\Lambda_{b}$ is likely to have another hadron in the other hemisphere, even if the sign of the $\Lambda_{b}$ is determined only by its unmodeled polarization, the other hemisphere is likely to cancel the information. A conservative estimate of this systematic error is $(14 \%)(0.23)(0.50)(0.50)=0.8 \%$ (relative), taking into account the branching fraction, expected polarization, polarization information dilution in hadronic $\Lambda_{b}$ decays, and charge dilution of $B$ baryons relative to the meson in the opposite hemisphere.

\subsubsection{Monte Carlo Statistics}

The finite sample of Monte Carlo available for this analysis incurs an error on the measurement. While it is statistical in nature, this error is best classified as a systematic error because it enters through the modeling necessary to extract $A_{b}$ from the data.

The Monte Carlo statistical error can best be evaluated by adding the MC statistical errors to the data statistical errors in quadrature before performing the fit of Equation 6.22. Subtracting of in quadrature the statistical error in the fit from the data errors alone yields the Monte Carlo statistical error, estimated to be a relative $1.64 \%$.

\subsection{Cross-Checks}

Among the most serious errors above are the $B$ fragmentation and decay modeling: and the light-quark subtraction. While the momentum-weighting exponent $\kappa$ and the parameters of the $B$ tag have been chosen to minimize the measurement error, it is a useful cross-check to vary these parameters of the analysis to see if any dependence of the answer appears.

\subsubsection{Kappa dependence}

At a fixed, very pure tag, the check of $\kappa$-dependence of the answer is a sensitive test of the validity of the fragmentation and decay model. For $\kappa \sim 0$, the technique simply counts the positively and negatively charged tracks in each hemisphere, subtracts them, and assigns the $b$ quark direction to point along the direction with the largest 


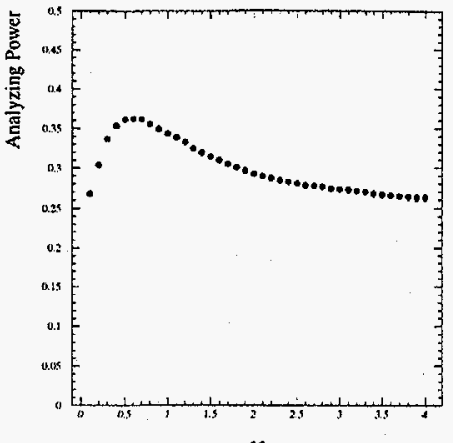

$\kappa$

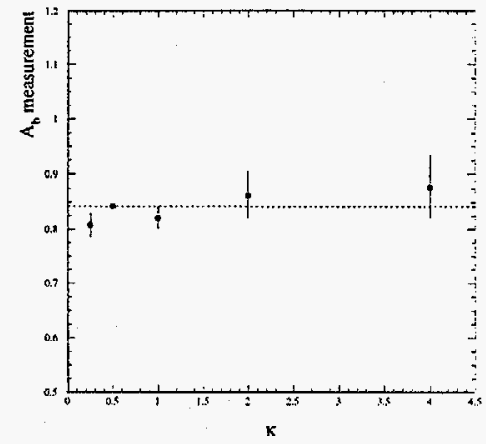

Figure 6.20: Analyzing power $A P$ in fully simulated Monte Carlo as a function of the momentum-weighting exponent $\kappa$.
Figure 6.21: Dependence of the value of $A_{b}$ measured with the binned asymmetry fit on the momentum-weighting exponent $\kappa$. Correlated statistical and systematic errors have been removed, taking $\kappa=0.5$ as a reference point.

count of negative tracks. In the limit as $\kappa \rightarrow \infty$, the momentum-weighted charge sum in 6.2 is dominated by the single most energetic track in the event. Because of the relatively low momentum of fragmentation tracks and the stiff fragmentation function of the $B$ mesons, the low- $\kappa$ limit probes the hadronization model, while the high- $\kappa$ limit probes the details of $B$ decay.

The Monte Carlo expectation of the $\kappa$-dependence of the analyzing power, defined in Equation 6.3, is shown in Figure 6.20, demonstrating that the different processes active in $B$ production and decay have different effects on the analyzing power.

Experiments at the $Z^{0}$ are uniquely qualified at this point in time to study the charge-momentum correlations in $B$-decay, owing to the clean separation of the decay products of the two $B$ hadrons. Experiments in which a $\Upsilon(4 S)$ is produced at rest for the most part confuse the decay products of the two $B$ hadrons and therefore cannot do any charge-dependent correlations. Some progress has been made in that realm with double-tagged semileptonic decays as well as with fully reconstructed $B$ decays.

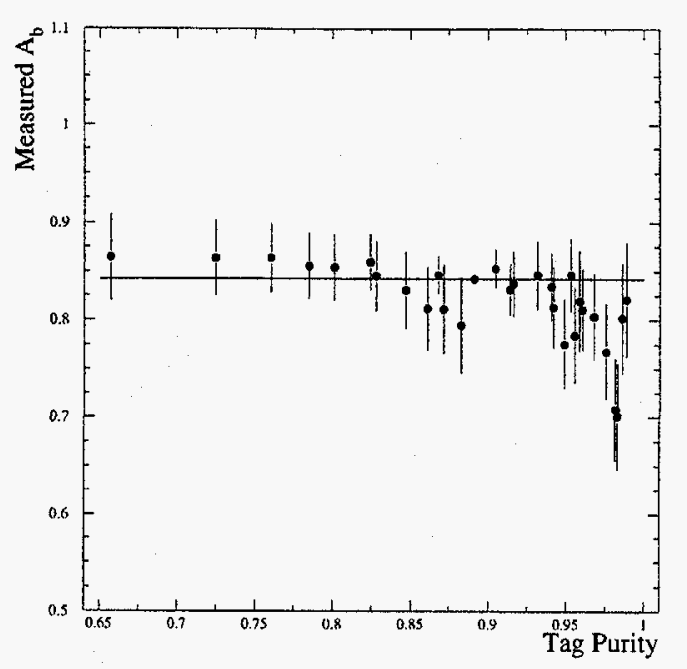

Figure 6.22: Tag dependence of the binned asymmetry $A_{b}$ measurement. The weakest tag is 2 tracks at $2 \sigma$, while the strongest tag is 4 tracks at $9 \sigma$. Correlated statistical and systematic errors have been removed, taking the 3 tracks at $3 \sigma$ point (with horizontal line) as a reference.

but that information cannot be used in an inclusive analysis such as this one.

The $\kappa$-dependence of the measured $A_{b}$ is shown in Figure 6.21. The flatness of this graph can in fact be used to constrain some of the details of the $B$-decay model in the Monte Carlo.

\section{4:2 Tag dependence}

Another important cross-check is to repeat the analysis at a fixed $\kappa$ but to vary the tag parameters. Because the asymmetry of the tag contamination is subtracted off using a Monte Carlo model, and because the background asymmetry has opposite sign from the signal asymmetry, this is a sensitive test of the ability of the Monte 
Carlo to simulate the background properly.

Also tested is the tag bias on the analyzing power. The tag is more efficient for events with many high-energy charged tracks in them, as the errors in the tracks: impact parameters are smaller and the tag becomes easier to satisfy. Events with large amounts of visible energy in charged tracks are expected to have more analyzing power than those whose distributions fluctuated more towards neutral energy. The tag efficiency depends most strongly, however, on the decay times of the $B$ hadrons, though, and the analyzing power depends only very weakly on those decay times, and that dependence comes from time-dependent mixing, and the mixing systematic error is small.

\subsubsection{Hemisphere consistency}

A feature of $Z^{0} \rightarrow b \bar{b}$ decays is that there are two $b$-type quarks in the final state; each event effectively has two chances to get the proper sign. More importantly, though. a comparison of the consistency between the signing of the two quarks using momentumweighted track charge can be used to determine how often the signing procedure yields the correct answer. The technique is to form the hemisphere momentum-weighted charges and see if they have the same sign or different sign. If both hemispheres' charges are signed correctly, then they should have oppositely signed charges. The relevant quantity to form then is

$$
H=P_{\text {agree }}-P_{\text {disagree }}
$$

where $P_{\text {agree }}$ is the fraction of tagged events with hemispheres of opposite charge, and $P_{\text {disagree }}$ is the fraction of events with the same sign charge. The hemisphere analyzing power $A P_{\text {hem }}$ is then given by

$$
A P_{h e m}=\sqrt{H},
$$

and can be checked for consistency between data and Monte Carlo. This variable is not quite the analyzing power for an analysis using only hemisphere charges owing to additional correlations between the two hemispheres, but it is a useful cross-check on the modeling of the Monte Carlo. It is the separate information from the hemisphere charges that allows the self-calibrated analysis of the next chapter to be performed.
Table 6.10: Asymmetries in the consistent-inconsistent hemisphere fractions, as defined in Equation 6.38 for tagged data and $\mathrm{MC}$ at different values of $\kappa$. Errors are statistical. A low values of $\kappa$, hemisphere correlations become more important, enlarging the fraction of events with agreeing hemispheres without increasing the analyzing power.

\begin{tabular}{|ccc|}
\hline$\kappa$ & Data $H$ & Monte Carlo $H$ \\
\hline 0.25 & $(12.66 \pm 0.92) \%$ & $(13.44 \pm 0.52) \%$ \\
0.50 & $(10.06 \pm 0.92) \%$ & $(9.28 \pm 0.52) \%$ \\
1.00 & $(6.65 \pm 0.92) \%$ & $(7.15 \pm 0.52) \%$ \\
2.00 & $(4.87 \pm 0.92) \%$ & $(5.27 \pm 0.52) \%$ \\
4.00 & $(4.70 \pm 0.92) \%$ & $(5.05 \pm 0.52) \%$ \\
\hline
\end{tabular}

The results of the comparison between data and Monte Carlo are summarized below for events passing the 2-D normalized impact parameter tag, and at several different values of $\kappa$.

\subsection{Summary}

This chapter has presented a binned-asymmetry fit for $A_{b}$ using the polarized forwardbackward asymmetry of lifetime-tagged $Z^{0} \rightarrow b \bar{b}$ events signed with momentumweighted track charge. The calibration of the tag composition, correct-sign probability, and radiative effects have been estimated with the SLD Monte Carlo. The resulting $A_{b}$ is found to be

$$
A_{b}=0.828 \pm 0.054 \text { (stat.) } \pm 0.070 \text { (syst.) }
$$

with the systematic error dominated by uncertainties in the details of $B$ fragmentation and decay, as well as modeling the tracking efficiency. The next chapter presents a technique designed to overcome these modeling errors by reducing the dependence on the Monte Carlo for calibration. 


\subsection{General Formulation}

The technique of maximum likelihood is described in many standard texts on statistics [165][166], so only an outline of its features necessary for this analysis will be presented. Briefly put, the technique asks the question: "What is the most probable

\section{Chapter 7}

\section{$A_{b}$ with Momentum-Weighted Charge: Maximum Likelihood}

An improvement to the technique of the previous chapter is to take advantage of the value of $Q$, defined in Equation 6.2, on each event. If $|Q|$ is large, the probability of assigning the charge correctly is near unity, while very little information about the sign is given for $|Q|$ near zero. This leads us to incorporate this information in a maximum-likelihood analysis, which is able to make optimal use of the quantities measured in the data.

A more important feature of the analysis presented in this chapter is that the effectiveness of the momentum-weighted charge technique may be calibrated by taking advantage of the momentum-weighted charge separately in the two hemispheres of each event. This procedure is a natural extension of the hemisphere consistency crosscheck of Section 6.4.3. It reduces the model dependence of the measurement of $A_{b}$. which, as indicated in Chapter 6, would ultimately limit the measurement.

This chapter describes maximum likelihood techniques in general, the specific likelihood function used in this analysis, and the calibration of its input parameters. It then describes the extraction of $A_{b}$, the remaining model dependencies, and crosschecks that have been performed. value of the parameters of the model given the data observed, and given no other a priori knowledge of the measurement?" The set of parameters defining a model to describe the data will be denoted $\left\{\xi_{i}\right\}^{*}$. The probability of the set of $\xi_{i}$ as a function of the observed data is often not known, but one can usually construct the probability of observing a data sample given $\xi_{i}$. Bayes's theorem allows the calculation of the probability:

$$
p\left(\left\{\xi_{i}\right\} \mid \text { data }\right)=\frac{p\left(\left\{\xi_{i}\right\}\right) p\left(\text { data } \mid\left\{\xi_{i}\right\}\right)}{p(\text { data })} .
$$

With no a priori biases, $p\left(\xi_{i}\right)$ is the same for all hypotheses. The denominator, $p($ data $)$, the likelihood of observing the data, does not depend on the $\xi_{i}$ and is unimportant in what follows. One may find the optimal $\left\{\xi_{i}\right\}$ by maximizing the probability of the data given $\left\{\xi_{i}\right\}$.

This probability function can be constructed out of multiplicative components for each event in the analysis:

$$
p\left(\operatorname{data} \mid\left\{\xi_{i}\right\}\right)=\prod_{\text {events }} p\left(\text { event },\left\{\xi_{i}\right\}\right)
$$

The mathematical tractability of this product is improved by converting it into a sum of logarithms:

$$
\ln \mathcal{L}=\sum_{\text {events }} \ln \left(p\left(\text { event },\left\{\xi_{i}\right\}\right)\right)
$$

Often it is $-\ln \mathcal{L}$ which is used in order to allow function minimization programs to be emptoyed. Statistical errors may be estimated from $\ln \mathcal{L}$ : the region of hypothesized parameter space within $1 \sigma$ of the fit value is that region for which $\ln \mathcal{L}$ is within 0.5 of its maximum value. In the case that there are several parameters to be fit, this will in general be an ellipsoid in that space, yielding both errors and statistical correlations.

\footnotetext{
"In our case, the only parameter we will fit for is $A_{b}$.
} 


\subsection{Likelihood Function}

The probability of observing an event as a function of its properties is given by the differential cross-section, Equation 1.61, with the $A_{f}$ as parameters to be measured or assumed. To be used as a likelihood function, though, the probability of mistagging or mis-signing an event must be incorporated. In addition, the effects of QCD radiation modify the observed distribution and must be included at this point. Overall scale factors on the differential cross-section, and even overall scale factors on individual events' probabilities do not enter, as they become constant offsets to $\ln \mathcal{L}$, and therefore affect neither the position of the maximum nor the width. The probability function used in this analysis is

$$
\begin{aligned}
& p\left(\text { event }_{i}, A_{b}, A_{c}, A_{b c k g}\right)=\left(1-A_{e} P_{e}^{i}\right)\left(1+\cos ^{2} \theta_{i}\right)+2\left(A_{e}-P_{e}\right) \cos \theta_{i}[ \\
& A_{b} f_{i}^{b}\left(2 p_{i}^{\text {correct }, b}-1\right)\left(1-\Delta_{Q C D, b}^{i}\right)+ \\
& A_{c} f_{i}^{c}\left(2 p_{i}^{\text {correct, }, c}-1\right)\left(1-\Delta_{Q C D, c}^{i}\right)+ \\
&\left.A_{b c k g}\left(1-f_{i}^{b}-f_{i}^{c}\right)\left(2 p_{i}^{\text {"correct", bckg }}-1\right)\right], \quad(7.4)
\end{aligned}
$$

where $A_{\mathrm{e}}$ is the asymmetry in electron coupling to the $Z^{0}, P_{\mathrm{e}}^{i}$ is the signed polarization of the electron beam when the event was recorded, $f_{i}^{b}$ is the probability that that event was a $Z^{0} \rightarrow b \bar{b}$ decay, and is parameterized in terms of the number of tracks missing the origin by $3 \sigma, f_{c}^{i}$ is a similar quantity for the $Z^{0} \rightarrow c \bar{c}$ probability, and $\Delta_{Q C D, b, c}^{i}$ are final-state QCD corrections [58][57], to be discussed later. $A_{b c k g}$ is an estimated asymmetry from $u \bar{u}, d \bar{d}$, and $s \bar{s}$ decays of the $Z^{0}$. The right-sign probabilities $p_{i}^{\text {correct, } b}$ and $p_{i}^{\text {correct,c }}$ are estimated as functions of the momentum weighted charge $|Q|$ of the event, defined in Equation 6.2. The $p_{i}^{\text {correct,b,c }}$ parameterize how well the algorithm signs the thrust axis on an event, and may be estimated from the Monte Carlo, but as will be described in Section 7.3, they can be inferred from the data with a much reduced model dependence.

It is interesting to note that $A_{e}$ appears in the probability function for the maximum likelihood technique, while it cancels from the left-right forward-backward asymmetry combination $\tilde{A}_{F B}$. In fact, it is easy to tell that its effect must vanish in the limit of a large data sample. If the experiment were run with $P_{e}= \pm 1$, then
$\left(A_{e}-P_{e}\right)=\mp\left(1-A_{e} P_{e}\right)$ and the dependence on $A_{e}$ vanishes entirely, because one can factor out arbitrary scale factors from the probability function for each event without affecting the answer. The experiment was run with $\left|P_{e}\right|<1$, though, but the observable asymmetry should scale simply with $P_{e}$, except for the fact that a finite data sample introduces statistical fluctuations. Any dependence on $A_{\varepsilon}$ in the final result with this technique will scale down with increasing data sample and will also become smaller as $\left|P_{e}\right| \rightarrow 1$.

\subsection{Calibrating the Analyzing Power}

The largest systematic errors in the binned asymmetry fit arise from using the Monte Carlo to estimate the correct-sign probability. The statistical error available with the 1994-1995 data is already smaller than the systematic error in the binned fit. The goal of this section is to find some observables available in the data that provide a calibration of $p^{\text {correct,b}}(|Q|)$. The technique used takes advantage of the fact that the two hemispheres give nearly independent estimations of the correct sign of the thrust axis. Presented below is a version of the analyzing power check of Section 6.4.3 which extracts maximal information by taking advantage of the values of the hemisphere charges instead of just their relative signs.

\subsubsection{Ansatz}

To compute $p^{\text {correct,b}}(|Q|)$, two assumptions must be made. First, an assumption of the functional form of the momentum-weighted charge distributions in the two hemispheres of an event must be made. The form chosen here is a Gaussian. The discussion below will be cast in terms of $Q_{b}$ and $Q_{\bar{b}}$, the momentum-weighted charges in the $b$ and $\bar{b}$ hemispheres. That sometimes the $b$ and $\bar{b}$ sometimes appear in the same hemisphere to complicate this assumption will be handled in Section 7.3.6. Relaxing the assumption that the distributions are Gaussian to determine systematic errors introduced by it will be described in Section 7.3.5. A Gaussian is likely to be a close representation of the $Q_{b}$ and $Q_{\bar{b}}$ distributions because the conditions of the central 
limit theorem [167] are nearly met. With a weighting exponent of $\kappa=0.5$, no single track dominates the $Q$ sum, and the mean $B$-decay multiplicity is $\sim 5.4$. For larger values of $\kappa$, single tracks become more important in the sum and the distribution becomes far less Gaussian. For $\kappa \rightarrow 0$, the distribution becomes discrete. For the case of a discrete distribution, the procedure for calibrating $p^{\text {correct, } b}$ can be performed without an assumption about the shape. The details will be given in Chapter 9 .

The second assumption necessary at this point is that the momentum-weighted charges in the $b$ and $\bar{b}$ hemispheres are statistically uncorrelated. It is important to separate the $b$ from the $\bar{b}$ hemisphere at this point, because the momentum-weighted charges in the two hemispheres of an event are most definitely correlated: if one hemisphere has a $b$, the other almost certainly will have $a \vec{b}$. It is this correlation which provides the signal for the calibration; correlation in addition to this can affect the technique's accuracy. Additional correlation can arise from charge conservation in the event and the requirement that both hemispheres be color singlets. The details of how these quantities are conserved affect the interhemisphere correlation. The definition and magnitude of this extra correlation will be discussed in Section 7.3.3.

These two assumptions can be formulated with

$$
p\left(Q_{b}, Q_{\bar{b}}\right)=p\left(Q_{b}\right) p\left(Q_{\bar{b}}\right),
$$

with

$$
p\left(Q_{b}\right)=\frac{1}{\sqrt{2 \pi \sigma_{0}^{2}}} e^{-\left(Q_{b}+Q_{0}\right)^{2} / 2 \sigma_{0}^{2}}
$$

and

$$
p\left(Q_{\bar{b}}\right)=\frac{1}{\sqrt{2 \pi \sigma_{0}^{2}}} e^{-\left(Q_{\bar{b}}-Q_{0}\right)^{2} / 2 \sigma_{0}^{2}}
$$

where $Q_{0}>0$. is the average momentum-weighted charge in the $\bar{b}$ hemisphere, and $\sigma_{0}$ is the single-hemisphere charge width.

A third assumption at this point is that the momentum-weighted charge distributions do not violate $C P$. While not strictly true for the $B$ hadrons [168], the decay modes with the largest $C P$-violating asymmetries have small branching fractions and do not affect the momentum-weighted charges because they are charge self-conjugate.
The most important $C P$-violating effect, though, is the presence of nuclear interactions of final-state particles with the detector material, adding extra positive charge on average to both the $b$ and $\bar{b}$ hemispheres. This effect can be measured with the data and it is discussed in Section 7.6. The $C P$ symmetry assumption appears in Equations 7.6 and 7.7 because

$$
\left\langle Q_{\bar{b}}\right\rangle=-\left\langle Q_{b}\right\rangle \equiv Q_{0} .
$$

The assumption of $C P$ symmetry will also constrain the possible form of the hemisphere correlations.

The Monte Carlo provides a guide as to how believable these two assumptions are. Shown in Figure 7.1 are the distributions for $Q_{b}$ and $Q_{\bar{b}}$, and their joint distribution. The distributions individually fit well to Gaussians. Their means are opposite at -0.57 and +0.69 , with widths of 2.79 and 2.78 , respectively. The Monte Carlo $B$ decay model manifestly conserves $C P$, and so the difference in means is entirely attributable to the simulation of nuclear scattering in the detector material. The interhemisphere correlation, to be defined and discussed in Section 7.3.3, is estimated to be $\sim 3 \%$.

\subsubsection{Correct-Sign Probability}

With the assumptions that the distributions are Gaussian and uncorrelated, the function $p^{\text {correct, } b}(|Q|)$ can be determined from the data. Convenient combinations of the hemisphere momentum-weighted charges are

$$
\begin{aligned}
& Q_{\text {sum }}=Q_{b}+Q_{\bar{b}}, \text { and } \\
& Q_{\text {diff }}=Q_{b}-Q_{\bar{b}} .
\end{aligned}
$$

The unsigned event momentum-weighted charge $|Q|$ used in Chapter 6 can be identified with $\left|Q_{\text {diff }}\right|$ defined above:

$$
\left|Q_{\text {diff }}\right|=|Q|=\left.\left|\sum_{\text {tracks }} q_{i}\right| \vec{p}_{i} \cdot \vec{t}\right|^{\kappa} \operatorname{sgn}\left(\vec{p}_{i} \cdot \vec{t}\right) \mid
$$

and henceforth will be referred to as $\left|Q_{\text {diff }}\right|$. Only the absolute value of $Q_{\text {diff }}$ is measurable because no knowledge of which hemisphere contains the $b$ and which the 

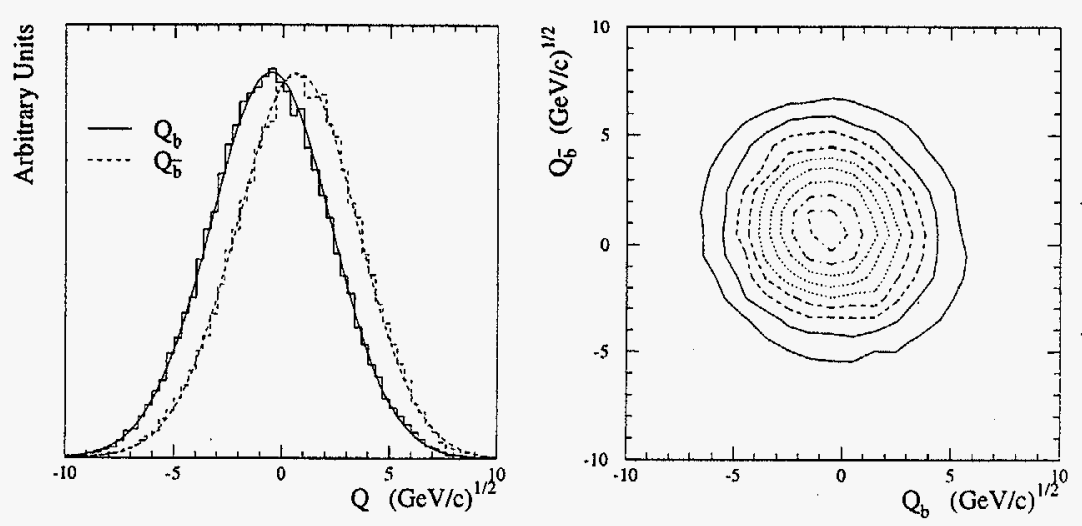

Figure 7.1: Monte Carlo distributions of $Q_{b}$ and $Q_{\bar{b}}$, with fits to a Gaussian hypothesis. The joint probability distribution has a correlation of $\sim 3 \%$.

$\bar{b}$ is assumed. The sum of the hemisphere charges, also mentioned in Chapter 6 , retains its definition:

$$
Q_{\text {sum }}=\sum_{\text {tracks }} q_{i}\left|\vec{p}_{i} \cdot \vec{t}\right|^{\kappa} .
$$

It can be directly measured in the data because it is invariant under an interchange of $b$ and $\bar{b}$.

The desired correct-sign probability is the probability $Q_{\text {diff }}$ has the sign expected from the charges of the $b$ and $\bar{b}$ :

$$
p^{\text {correct, } b}\left(\left|Q_{\text {diff } f}\right|\right)=p\left(Q_{\text {diff }}<0\right) \text {. }
$$

In terms of the Gaussian distributions of Equations 7.6 and 7.7 ,

$$
p\left(Q_{d i f f}\right)=\frac{1}{\sqrt{2 \pi \sigma_{Q_{d i f f}}^{2}}} e^{-\left(Q_{d i f f}+2 Q_{0}\right)^{2} / 2 \sigma_{Q_{d i f f}^{2}}^{2}}
$$

where

$$
\sigma_{Q_{\text {diff }}}=\sigma_{0} \sqrt{2}
$$

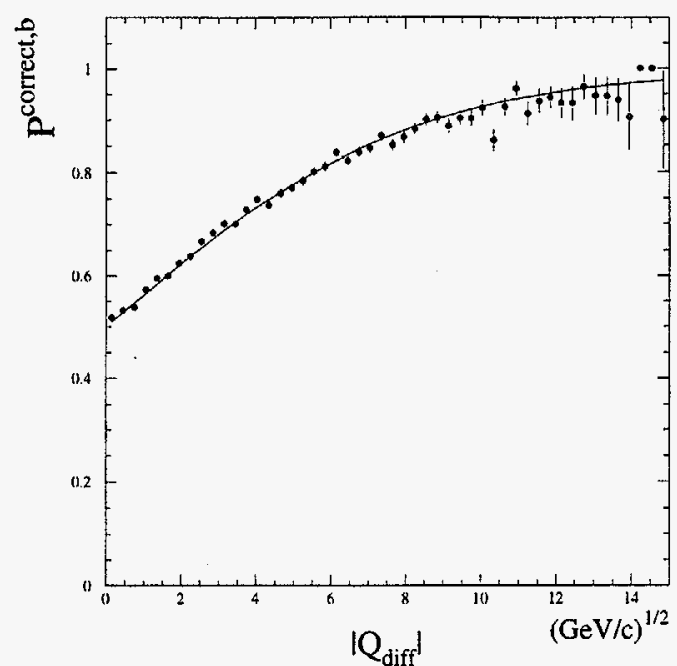

Figure 7.2: Correct-sign probability $p^{\text {correct,b }}$ as a function of $\left|Q_{\text {diff }}\right|$, as estimated from the Monte Carlo. The function appearing in Equation 7.16 with $\alpha_{b}=0.2505 \pm 0.0022$ $(\mathrm{GeV} / \mathrm{c})^{-1 / 2}$, where the error is statistical on the Monte Carlo.

is the width of the $Q_{\text {diff }}$ distribution. The correct-sign probability is then given by

$$
p^{\text {correct, },}\left(\left|Q_{\text {diff }}\right|\right)=\frac{p\left(Q_{\text {diff }}<0\right)}{p\left(Q_{\text {diff }}<0\right)+p\left(Q_{\text {diff }}>0\right)}=\frac{1}{1+e^{-\alpha_{b}\left|Q_{d i f f}\right|}},
$$

with

$$
\alpha_{b}=4 Q_{0} / \sigma_{Q_{d i f j}^{2}}^{2}
$$

The quantity $\alpha_{b}$ is a parameterization of how well the momentum-weighted charge technique signs the thrust axis. Larger values of $\alpha_{b}$ correspond to more reliable charge assignment, either due to a larger charge separation between $b$ and $\bar{b}$, or smaller statistical fluctuations of the measured charge. It is also the case that

$$
0.5 \leq p^{\text {correct,b}}\left(\left|Q_{\operatorname{dif} f}\right|\right) \leq 1
$$


The task then becomes how to calculate $\alpha_{b}$ from event observables. Equation 7.16 can be fit to the Monte Carlo, where the $b$ and $\bar{b}$ axes are known and a value of $\alpha_{b}$ can be fit, but the object is to reduce the Monte Carlo dependence. Fortunately, a comparison of the widths of the $\left|Q_{\text {sum }}\right|$ and $\left|Q_{\text {diff }}\right|$ distributions provides the required information. In terms of the Gaussians of Equations 7.6 and 7.7,

$$
p\left(Q_{\text {sum }}\right)=\frac{1}{\sqrt{2 \pi \sigma_{s u m}^{2}}} e^{-Q_{s u m}^{2} / 2 \sigma_{s u m}^{2}}
$$

where

$$
\sigma_{s u m}=\sigma_{Q_{\text {diff }}}=\sigma_{0} \sqrt{2}
$$

is the width of the $Q_{\text {sum }}$ distribution. The fact that $Q_{\text {sum }}$ and $Q_{\text {diff }}$ have the same width is a consequence of the assumption that $Q_{b}$ and $Q_{\bar{b}}$ are uncorrelated. Relaxing this assumption will be discussed in Section 7.3.3. Measuring the width of the $Q_{\text {sum }}$ distribution in the data yields $\sigma_{0}$ immediately.

The next step is to evaluate $Q_{0}$ from data observables. While the distribution of $Q_{\text {diff }}$ is Gaussian, the distribution of $\left|Q_{\text {diff }}\right|$ is the sum of two Gaussians ${ }^{t}$ :

$$
p\left(\left|Q_{d i f f}\right|\right)=\frac{1}{\sqrt{2 \pi \sigma_{Q_{d i} f}^{2}}}\left(e^{-\left(\left|Q_{d i f f}\right|-2 Q_{0}\right)^{2} / 2 \sigma_{Q_{d i f f}^{2}}^{2}}+e^{-\left(\left|Q_{d i f f}\right|+2 Q_{0}\right)^{2} / 2 \sigma_{Q_{d i f f}}^{2}}\right) .
$$

This sum of two Gaussian distributions can be approximated very well by a single Gaussian distribution, as long as $Q_{0} \ll \sigma_{0}^{\ddagger}$.

$$
p\left(\left|Q_{\text {diff } f}\right|\right) \simeq \frac{2}{\sqrt{2 \pi \sigma_{\left|Q_{\text {diff }}\right|}^{2}}} e^{-\left|Q_{\text {diff }}\right|^{2} / 2 \sigma_{\mid Q_{d i f f}}^{2}} .
$$

The width of this single distribution may be found by setting Equations 7.21 and 7.22 equal at $\left|Q_{\text {diff }}\right|=0$ and solving for $\sigma_{\left|Q_{\text {diff }}\right|}$, yielding

$$
\sigma_{\left|Q_{d i f f}\right|} \simeq \sigma_{Q_{d i f f}}\left(1+2 Q_{0}^{2} / \sigma_{Q_{d i f f}}^{2}\right)
$$

IIn the high statistics limit, if $Q_{\text {sum }}$ fits well to a Gaussian, $\left|Q_{d i f f}\right|$ will not. With the current data sample, however, the distributions of both $Q_{\text {sum }}$ and $\left|Q_{d i f f}\right|$ are indistinguishable from Gaussian.

IIn our case, $Q_{0} \simeq 0.99 \mathrm{GeV}^{1 / 2}, \sigma \simeq 2.60 \mathrm{GeV}^{1 / 2}$, and the single-Gaussian approximation to Equation 7.21 using the width of Equation 7.23 yields correct-sign probabilities which deviate by no more than $0.7 \%$ from what would be obtained without the approximation. This point will return again in Section 7.3.5. We will see that this approximation introduces a small bias to the technique which will be accounted for. again using the approximation that $Q_{0} \ll \sigma_{0}$. This result can also be obtained by setting the second derivative of Equations 7.21 and 7.22 equal at $\left|Q_{d i j f}\right|=0$.

The variable $\sigma_{\left|Q_{i i f f}\right|}$ is a measurable quantity; it is just the RMS deviation of $\left|Q_{\text {dijf }}\right|$ from zero. The width of $Q_{s u m}$ can also be found by its RMS deviation from zero, as it is expected to have zero mean. Using Equations 7.23 and $7.20, Q_{0}$ can be obtained in terms of observables $\sigma_{\text {sum }}$ and $\sigma_{\left|Q_{\text {diff }}\right|}$ :

$$
Q_{0}=\sqrt{\left(\sigma_{s u m} \sigma_{\left|Q_{d i f f}\right|}-\sigma_{s u m}^{2}\right) / 2}
$$

Combining Equations 7.24 and 7.20 with 7.17 , one obtains $\alpha_{b}$ in terms of data observables:

$$
\alpha_{b}=\frac{2 \sqrt{2\left(\frac{\sigma_{\left|Q_{\text {diff }}\right|}}{\sigma_{\text {sum }}}-1\right)}}{\sigma_{\text {sum }}} .
$$

The data used to calculate $\sigma_{\text {sum }}$ and $\sigma_{\left|Q_{\text {diff }}\right|}$ are shown in Figure 7.3.

Because $\alpha_{b}$ can be derived from observables in the data, the technique is called self-calibrating. Errors introduced by modeling tracking efficiency, $B$ decays, fragmentation, and mixing do not appear in the result because they are calibrated with this $\alpha_{b}$. It should be noted that only data events passing the 2-D normalized impact parameter tag are used to form $Q_{s u m}$ and $\left|Q_{\text {diff }}\right|$. In this manner, a very pure sample of $Z^{0} \rightarrow b \bar{b}$ events is used to calibrate its own analyzing power, and any biases introduced by the tag requirements are included in the calibration.

\subsubsection{Hemisphere Correlations}

The assumption that the distributions of $Q_{b}$ and $Q_{\bar{b}}$ are uncorrelated has already been shown to be almost, but not quite, true. Deviations from an uncorrelated distribution will modify the expected value of $\alpha_{b}$ as obtained from $\sigma_{\text {sum }}$ and $\sigma_{\left|Q_{\text {diff }}\right|}$.

The joint probability distribution of $Q_{b}$ and $Q_{\bar{b}}$, in the absence of correlation, is a round, symmetric, two-dimensional Gaussian distribution (Equations 7.5-7.7). Correlations will stretch this distribution along some axis. Because the widths of the projections along the $Q_{b}$ and $Q_{\vec{b}}$ axes must be equivalent by $C P$ symmetry, the axis of deformation must be along the $45^{\circ}$ axis shown in Figure 7.4. The correlation can 


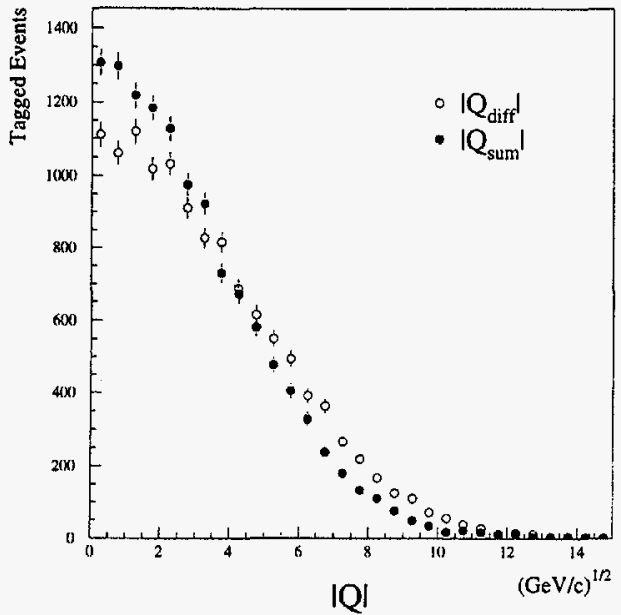

Figure 7.3: Distributions of the hemisphere charge sums and differences for 1993-1995 data with $\kappa=0.5$.

then be defined to be

$$
\lambda \equiv \frac{\sigma_{\text {diff }}}{\sigma_{\text {sum }}}-1
$$

For the fully simulated SLD Monte Carlo $Z^{0} \rightarrow b \bar{b}$ sample, $\lambda=2.9 \%$.

To incorporate $\lambda$ into the calculation of the analyzing power parameter $\alpha_{b}$, one notes that the right-sign probability $p^{\text {correct,b}}\left(\left|Q_{\text {diff }}\right|\right)$ depends only on modeling the probability distribution projected along the $Q_{\text {diff }}$ direction, as can be seen from Equations 7.16 and 7.17. It is $\sigma_{\text {diff }}$, therefore, which is to be used in Equation 7.25. But $\sigma_{\text {sum }}$ is measured, so a multiplicative correction of $(1+\lambda)$ must be applied before using it to find $\alpha_{b}$ :

$$
\alpha_{b}=\frac{2 \sqrt{2\left(\frac{\sigma_{\left|Q_{\text {diff }}\right|}}{(\mathfrak{1}+\lambda) \sigma_{s u m}}-1\right)}}{(1+\lambda) \sigma_{\text {sum }}} .
$$

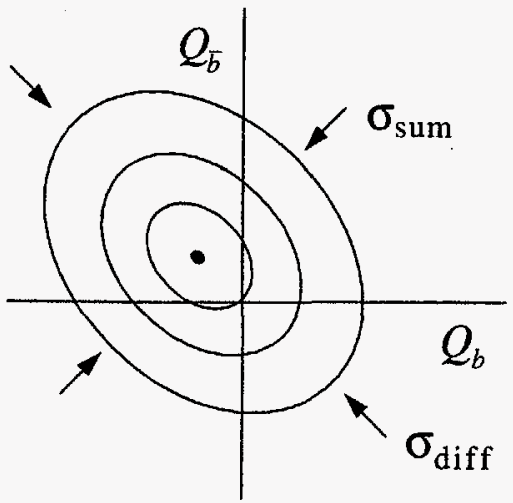

Figure 7.4: Possible effect of correlation on $\mathrm{p}\left(Q_{b}, Q_{\bar{b}}\right)$. CP symmetry requires that the major and minor axes of the ellipse be oriented at $45^{\circ}$ to the $Q_{b}$ and $Q_{\bar{b}}$ axes.

The magnitude of the correlation is difficult to estimate from the data itself, because a lengthening of the joint-probability distribution along the $Q_{\text {diff }}$ axis has the same effect on $Q_{\text {diff }}$ as an increased charge separation from a larger $Q_{0}$. There is, however, a pair of observables in each event that does in fact carry the appropriate information, $\left|Q_{b}\right|$ and $\left|Q_{\bar{b}}\right|$, although it cannot be determined which is which. Nonetheless, if the two unsigned hemisphere charges have some correlation, it can only arise from $\lambda$ and not $Q_{0}$. This technique has not been pursued here because it is not statistically powerful enough - one would need a sample of tagged events four times as large than the one at hand in order to measure the correlation to an absolute precision of $\pm 1 \%$, which would be a $30 \%$ relative error on $\lambda$, the uncertainty incurred in this analysis by using the Monte Carlo to estimate $\lambda$. This uncertainty is estimated by using different Monte Carlo models - JETSET 7.4 [30][31], HERWIG 5.7 [169], and JETSET's independent jet model - to calculate the correlation. 


\subsubsection{Light-Flavor Subtraction}

A concern about using the widths of the momentum-weighted charge distributions to calibrate the analyzing power is that the tagged sample is not a pure sample of $Z^{0} \rightarrow b \bar{b}$. The light-flavor contamination must be accounted for using a Monte Carlo model. Fortunately, though, given the nature of the widths of $Q_{\text {sum }}$ and $Q_{\text {diff }}$, the light-quark fractions subtract off in quadrature and the final answer has very little dependence on the details of this subtraction.

The widths $\sigma_{\text {sum }}$ and $\sigma_{\left|Q_{d i f f}\right|}$ are calculated by finding the RMS deviations of their respective distributions from zero:

$$
\sigma_{s u m}=\sqrt{\frac{1}{N} \sum_{\substack{\text { tagged } \\ \text { events }}} Q_{\text {sum }}^{2}}
$$

and

$$
\sigma_{\left|Q_{\text {diff }}\right|}=\sqrt{\frac{1}{N} \sum_{\substack{\text { tagged } \\ \text { events }}} Q_{\text {diff }}^{2}}
$$

$N$ is the number of tagged events. These expressions can be broken down into their flavor components and the light flavor parts subtracted off to give widths that correspond only to the $Z^{0} \rightarrow b \bar{b}$ sample:

$$
\sigma_{s u m}^{b}=\left[\frac{\sigma_{s u m, o b s}^{2}-\Pi_{c} \sigma_{s u m, c}^{2}-\Pi_{u d s} \sigma_{s u m, u d s}^{2}}{\Pi_{b}}\right]^{\frac{1}{2}},
$$

and

$$
\sigma_{\left|Q_{d i f f}\right|}^{b}=\left[\frac{\sigma_{\left|Q_{d i f f}^{2}\right|, o b s}-\Pi_{c} \sigma_{\left|Q_{d i f f}\right|, c}^{2}-\Pi_{u d s} \sigma_{\left|Q_{d i f f}\right|, u d s}^{2}}{\Pi_{b}}\right]^{\frac{1}{2}}
$$

where $\sigma_{s u m, o b s}$ is the width of the sum distribution measured in the data, $\sigma_{\text {sum,c }}$ and $\sigma_{\text {sum, } u d s}$ are the charm and $u d s$ widths estimated from Monte Carlo, respectively, and $\Pi_{b}, \Pi_{c}$, and $\Pi_{u d s}=1-\Pi_{b}-\Pi_{c}$ are the contributions to the tag of $b, c$, and $u d s$ events.

The details of the light-flavor subtraction are shown in Table 7.1. It can be seen that the Monte Carlo charm widths are very similar to the data widths, and therefore do not change the average width by much in the corrections 7.30 and 7.31 . The total
Table 7.1: Contributions to the light-flavor subtraction to the data $\sigma_{\text {sum }}$ and $\sigma_{\left|Q_{\text {drfs }}\right|}$. The "All Data" row is included for comparison. The widths have not been corrected for correlations. Errors are statistical only.

\begin{tabular}{|lccc|}
\hline Sample & $\sigma_{\text {sum }}(\mathrm{GeV} / \mathrm{c})^{\frac{1}{2}}$ & $\sigma_{\mid Q_{\text {di } f f \mid}}(\mathrm{GeV} / \mathrm{c})^{\frac{1}{2}}$ & Contribution to Tag \\
\hline Tagged Data & $3.673 \pm 0.024$ & $4.200 \pm 0.027$ & $100 \%$ \\
All Data & $3.684 \pm 0.009$ & $4.322 \pm 0.013$ & - \\
MC $Z^{0} \rightarrow b \bar{b}$ & $3.797 \pm 0.011$ & $4.302 \pm 0.013$ & $89 \%$ \\
MC $Z^{0} \rightarrow c \bar{c}$ & $3.671 \pm 0.012$ & $4.113 \pm 0.014$ & $10 \%$ \\
MC $Z^{0} \rightarrow u d s$ & $3.742 \pm 0.008$ & $4.220 \pm 0.010$ & $1 \%$ \\
\hline
\end{tabular}

effect on $\alpha_{b}$ is a relative shift of $0.34 \%$ and is included in the systematic errors with $100 \%$ of the correction in order to be conservative.

\subsubsection{Checking the Gaussian Hypothesis}

The Gaussian hypothesis of Equations 7.6 and 7.7 is supported by the Monte Carlo. but the sensitivity of the analysis to the shape of $Q_{b}$ and $Q_{\bar{b}}$ needs to be estimated in the case that it deviates from being Gaussian.

The technique used to determine this sensitivity is to generate a large quantity of "toy" Monte Carlo which simulates events using the differential cross-section of Equation 1.61 at a fixed value of $A_{b}$ and $P_{\epsilon}$. Each event is given uncorrelated values of $Q_{b}$ and $Q_{\bar{b}}$, distributed according to a probability function which is chosen arbitrarity: The constraints on this distribution are that

$$
p\left(Q_{b}\right)=p\left(-Q_{\bar{b}}\right)
$$

and the width of $Q_{\text {sum }}$ and $\left|Q_{\text {diff }}\right|$ match those measured in the data.

Four different probability distributions were chosen, shown in Figure 7.5. The events generated with these distributions were then analyzed with the same selfcalibrated maximum-likelihood technique as was used with the data, with the exception that the correlation $\lambda$ was set to zero. Table 7.2 shows the estimated biases to 
Table 7.2: Fit biases estimated from a Monte Carlo simulation with $Q_{b}$ generated with different distributions. A positive fit bias means that the self-calibrated technique would overestimate the true asymmetry if the $Q_{b}$ distribution had the hypothetical shape given.

\begin{tabular}{|lcc|}
\hline Trial Distribution & Fit Bias $\left(\triangle A_{b} / A_{b}\right)$ & $\chi^{2}(D O F=12)$ \\
\hline Gaussian & $+(0.57 \pm 0.01) \%$ & 15.24 \\
Isosceles Triangular & $-(0.4 \pm 0.1) \%$ & 279.6 \\
Right Triangle (L) & $-(2.2 \pm 0.2) \%$ & $588.0^{* *}$ \\
Right Triangle (R) & $+(2.4 \pm 0.2) \%$ & $588.0^{* *}$ \\
Double Gaussian & $+(1.96 \pm 0.1) \%$ & 328.8 \\
\hline
\end{tabular}

the fit when the different probability distributions of Figure 7.5 were used.

The best observable quantity which can be used to constrain the underlying momentum-weighted charge distribution is $p\left(\left|Q_{h e m}\right|\right)$, where $Q_{h e m}$ is the momentumweighted charge in a single hemisphere ${ }^{\S}$. The absolute value for the hemisphere charge needs to be taken because it is unknown which hemisphere contains the $b$ and which the $\bar{b}$. For each of the probability distributions in Figure 7.5, a functional form can be derived for $p\left(\left|Q_{h e m}\right|\right)$, and these fit to the data distributions. The $\chi^{2}$ per degree of freedom is listed for each of these, ruling out all but the Gaussian distribution at high confidence.

It was discovered with a high-statistics toy Monte Carlo run that if the moment umweighted charge distributions are genuinely Gaussian, then there is a small bias in the fit answer, listed in Table 7.2. This correction, which is due to the approximation of Equation 7.22, will be applied to the final result, as well as on the Monte Carlo cross-check of Section 7.4 and the normalized momentum-weighted charge analysis of 7.7 .

\footnotetext{
${ }^{5}$ The distribution of $Q_{s u m}$ is also a discriminator, but is less powerful because the distribution of the sum of two randomly distributed independent variables will in general fit better to a Gaussian than either of the components of the sum, by the Central Limit Theorem.

"* The asymmetric triangle fits to $p\left(\left|Q_{h e m}\right|\right)$ are optimal when the vertical side of the triangle is at $Q_{\text {hem }}=0$, which would imply $100 \%$ analyzing power. For a more realistic analyzing power, the chisquared of these fits rises to 3,300 , with 12 degrees of freedom.
}

a)

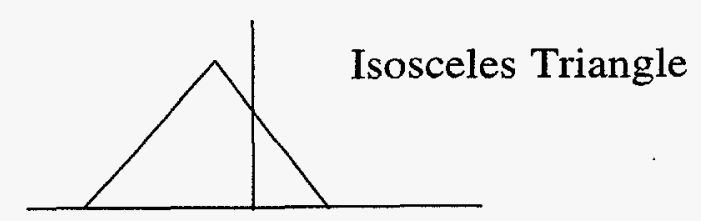

b)

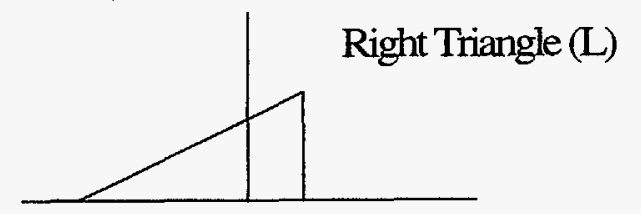

c)

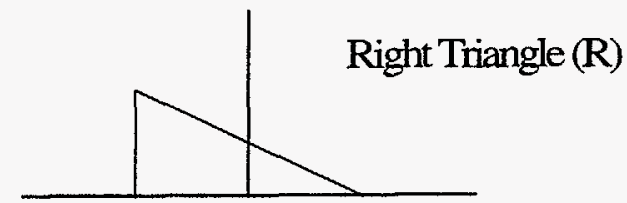

d)

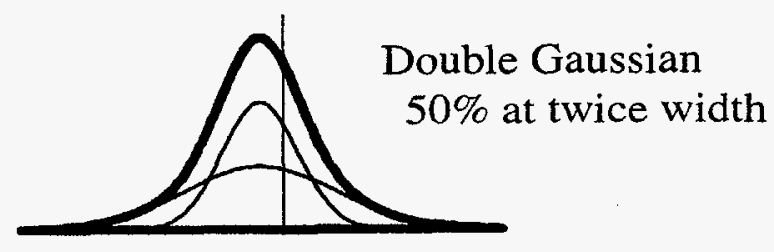

Figure 7.5: Different hypothetical $Q_{b}$ distributions used to test the sensitivity of the fit to $p\left(Q_{b}\right)$. Each distribution is given two degrees of freedom - the mean and the width. 


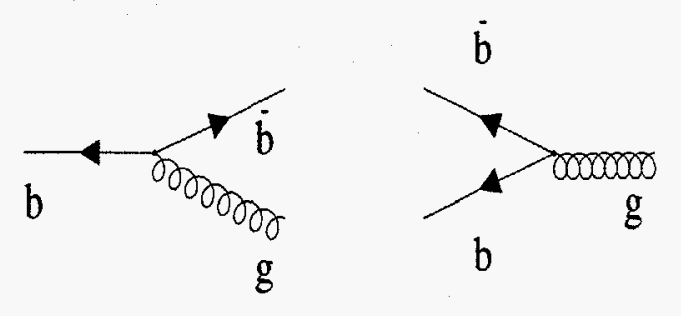

Figure 7.6: Gluon bremsstrahlung smearing the angle of the final-state quark (left): and backscattering it into the opposite hemisphere (right).

\subsubsection{QCD Corrections}

Corrections due to hard gluon radiation, discussed in Sections 1.5 and 6.3.1, are applied with the $\cos \theta$-dependent $1-\Delta_{Q C D}^{i}$ terms in the likelihood function of Equation 7.4. The self-calibrated technique introduces one complication to $\triangle_{Q C D}$, though, in that part of the correction is included in the self-calibration technique.

Most of the asymmetry dilution due to hard gluon radiation comes from smearing the quark axis before its detection. Using the thrust axis reduces the effect somewhat because the thrust axis is a more reliable indicator of the decay axis because it favors the fastest jet in the event, which has usually undergone less gluon radiation than an average jet. A second component of the QCD correction comes from events in which the gluon radiation is so hard that the two quarks end up in the same hemisphere. This affects not just the ability to find the angle, but the ability to sign the event correctly. The event-signing probability is calibrated by $\sigma_{s u m}$ and $\sigma_{\left|Q_{d i f f}\right|}$, but the angular smearing is not. The two contributions are illustrated in Figure 7.6. Figure 6.19 itemizes, using the JETSET 7.4 generator, the effects of these two contributions.

\subsection{Likelihood fit to MC}

A test of bias inherent in the self-calibrated maximum-likelihood fit is to generate fully simulated Monte Carlo and analyze it as if it were the data. Because the Monte Carlo is used to estimate the light flavor contamination in the tag, the errors arising from tag contamination vanish in this comparison. The Monte Carlo was generated with $A_{b}=0.9357$, and the value extracted from the Monte Carlo using this technique is $A_{b}^{M C \text {, meas }}=0.950 \pm 0.020$ (stat) \pm 0.014 ( $\alpha$ stat).

A more serious test of the calibration and fit is to alter the analyzing power in the Monte Carlo and to re-fit for $A_{b}$. This test was performed with an additional tracking inefficiency of $20 \%$ applied randomly to the Monte Carlo. If this simulation were used to estimate $A_{b}$ using the binned asymmetry fit, the value of $A_{b}$ would change by a relative $40 \%$, assuming that the corrections are still linear. The inefficient Monte Carlo is used to calibrate its own analyzing power using $\sigma_{\text {sum }}$ and $\sigma_{\left|Q_{\text {diff }}\right|}$, and the fit result is $A_{b}^{M C \text {,meas }}=0.991 \pm 0.032$ (stat) \pm 0.061 ( $\alpha$ stat). These tests demonstrate that the technique is unbiased and that it produces a reliable result regardless of the analyzing power.

\subsection{Measurement of $A_{b}$ with Maximum Likelihood Track Charge}

The measurement of $A_{b}$ puts together the pieces described in Sections 7.1-7.3. First, events are tagged with the normalized 2-D impact parameter tag, retaining only those events with more than 3 tracks missing the origin by $+3 \sigma$, the same tag used in the previous analysis. The quantities $\sigma_{\text {sum }}$ and $\sigma_{\left|Q_{\text {diff }}\right|}$ are measured in the tagged data sample. The Monte Carlo is then used to estimate $\lambda$, as well as the light-flavor contributions to the the sum and difference widths. The data widths are corrected for the tag contamination using Equations 7.30 and 7.31. The value of $\alpha_{b}$ is then computed for the data with Equation 7.27, using the Monte Carlo correlation $\lambda$. The effect of hard gluon radiation is accounted for with $\Delta_{Q C D}(\cos \theta)$, calculated in [58]. Systematic errors from this correction will be discussed in Section 7.6.9. 
Because the tracking acceptance is not uniform in polar angle, $\alpha_{b}$ is expected to be a function of $\cos \theta$. This dependence is estimated using the Monte Carlo, while the average value of $\alpha_{b}$ is scaled to match the data. The procedure is to compute in the Monte Carlo the correct-sign probability of tagged $Z^{0} \rightarrow b \bar{b}$ events as a function of $\left|Q_{\text {diff } f}\right|$ and fit an $\alpha_{b}$ in each of ten bins of $\cos \theta$, as well as in the aggregate $|\cos \theta|<0.7$. The ratio $\alpha_{b, d a t a} / \alpha_{b, M C}^{|\cos \theta|<0.7}$ is used to scale the $\cos \theta$-dependent $\alpha$ values obtained from the Monte Carlo. It is these values that are used to estimate $p^{\text {correct,b}}\left(\left|Q_{\text {diff }}\right|\right)$ in the likelihood function.

The other ingredients to the likelihood function are taken from the Monte Carlo simulation. The flavor probabilities $f^{b}$ and $f^{c}$ are estimated as functions of the number of tracks with significant impact parameters and read from of Figure 6.11. The analyzing power function for charm is estimated from the Monte Carlo by forming $p^{\text {correct,c }}\left(\left|Q_{\text {diff }}\right|\right)$ for $Z^{0} \rightarrow c \bar{c}$ events and fitting for $\alpha_{c}(\cos \theta)$ using Equation 7.16. The QCD correction applied to the charm portion of the likelihood function is discussed also in Reference [58] and are somewhat larger than for the $b$ case. The value of $A_{c}$ is fixed at its Standard Model value of 0.67 , which is within errors of the current LEP measurement of $0.65 \pm 0.05$, found by combining the LEP measurement of $A_{F B}^{a_{c}}=$ $0.0729 \pm 0.0058[64]$ with the combined LEP + SLD $A_{e}$ measurements, as described in Chapter 2. The reason not to fit simultaneously for $A_{c}$ in this analysis is that the hard tag requirement leaves only a $10 \%$ residue of charm contamination in the sample. If the tag is weakened to include events with fewer significant tracks to allow a larger charmed sample, then $A_{c}$ could indeed be measured, although its value would depend on modeling of $\alpha_{c}$, which is estimated from Monte Carlo and therefore has large modeling uncertainties. In addition, the fraction of $u d s$ events in the weaker tags is not well understood.

The likelihood sum over all tagged events as a function of $A_{b}$ is shown in Figure 7.7 . Its attains its maximum value at $A_{b}=0.851 \pm 0.046$, where the statistical error is estimated by finding the interval in $A_{b}$ for which $\ln \mathcal{L}_{\max }-\ln \mathcal{L}<0.5$, by fitting a parabola to the likelihood function. The resulting fit value must be corrected for the estimated fractional technique bias of $-0.57 \%$, discussed in Section 7.3 .5 , as well as QED radiative effects, amounting to a fractional $+0.17 \%$ described in Section 1.5 .
Table 7.3: Details of the momentum-weighted charge maximum-likelihood fit for $A_{b}$.

\begin{tabular}{|lcc|}
\hline & Data & Monte Carlo \\
\hline$\sigma_{\text {sum }}(\mathrm{GeV} / \mathrm{c})^{\frac{1}{2}}$ & $3.673 \pm 0.024$ & $3.797 \pm 0.011$ \\
$\sigma_{\left|Q_{\text {diff }}\right|}(\mathrm{GeV} / \mathrm{c})^{\frac{1}{2}}$ & $4.200 \pm 0.027$ & $4.302 \pm 0.013$ \\
$\alpha_{b}(\mathrm{GeV})^{-\frac{1}{2}}$ & $0.253 \pm 0.013$ & $0.245 \pm 0.005$ \\
$\lambda$ & Same as MC & $2.85 \%$ \\
Measured $A_{b}$ & $0.848 \pm 0.046$ (stat.) & $0.950 \pm 0.020$ (stat.) \\
$\delta A_{b} / A_{b}$ from $\alpha_{b}$ statistics & $3.4 \%$ & $1.5 \%$ \\
\hline
\end{tabular}

A further correction arising from the b-quark's $\beta<1$, described in Equation 1.60 . lowers the measured value of $A_{b}$ by a relative $0.02 \%$. The corrected value obtained is then $A_{b}=0.848 \pm 0.046$ (stat.).

\subsection{Systematic Error Analysis}

\subsubsection{Statistical Error on $\alpha_{b}$}

The dependence of the measured $A_{b}$ on $\alpha_{b}$ was determined by re-analyzing the data at several different values of $\alpha_{b}$. The same procedure was applied to find the dependence on $\alpha_{c}$.

$\frac{\partial A_{b}}{\partial \alpha_{b}}=-1.944$

and

$$
\frac{\partial A_{b}}{\partial \alpha_{c}}=-0.209 \text {. }
$$

The statistical error on $\alpha_{b}$ was propagated from the statistical errors on $\sigma_{\text {sum }}$ and $\sigma_{\left|Q_{\text {diff }}\right|}$, and appears as an error on $A_{b}$. This error is classified as a systematic error because it is a model of the analyzing power, even if the information for it comes from the data. This error is expected to diminish in proportion to $1 / \sqrt{N}$. 


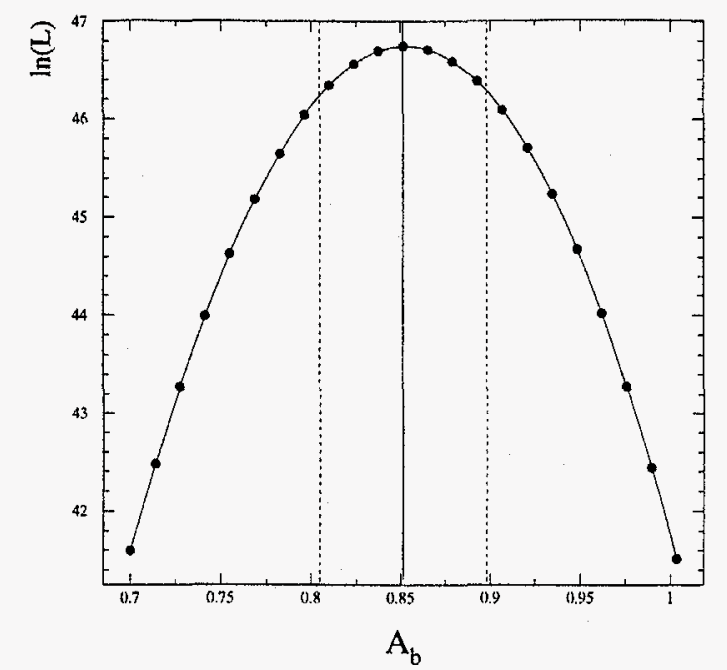

Figure 7.7: Log likelihood as a function of $A_{b}$, shown fitted to a parabola. The maximum occurs for $A_{b}=0.851 \pm 0.046$.

\subsubsection{Shape of the $Q_{b}$ distribution}

The different $Q_{b}$ shapes investigated in Section 7.3.5 show a somewhat wider distribution of estimated biases than is likely to be the case for the data, indicated by the fact that none of the trial functions fits the data. A conservative estimate of the systematic error from this contribution is $1.0 \%$.

\subsubsection{Angular Dependence of $\alpha_{b}$}

Another entry for model dependence is the use of the Monte Carlo to estimate the angular dependence of $\alpha_{b}$. Because the analyzing power of the technique is expected to fall with increasing $\cos \theta$ because of loss of tracking outside the detector acceptance, other functional forms of $\alpha_{b}(\cos \theta)$ can be used if they are related to tracling

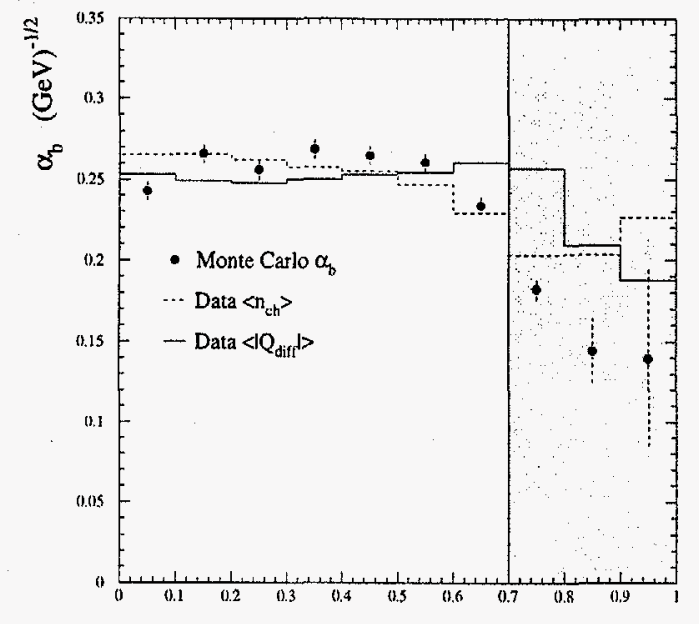

$\left|\cos \theta_{1}\right|$

Figure 7.8: Trial shapes for the $\cos \theta$-dependence of $\alpha_{b}$. Points are the $\mathrm{MC}$ estimate: the dashed line is the average multiplicity in hadronic events, and the solid line is $\left\langle\left|Q_{d i f f}\right|\right\rangle$ in hadronic events. Each curve has been scaled to the data $\alpha_{b}$. The shaded region is excluded by the analysis cut.

observables in the data. The functions tried are the average multiplicity versus $\cos \theta$ for all selected hadronic events, and also the average value of $\left|Q_{\text {diff } f}\right|$ for all selected hadronic events. These distributions may be scaled to the data $\alpha_{b}$ in the same way the Monte Carlo $\cos \theta$-dependent $\alpha_{b}$ was, and the variations provide a conservative estimate of the systematic error. from using the Monte Carlo shape of $\alpha_{b}(\cos \theta)$.

\subsubsection{Interhemisphere Correlation}

The correlation between the momentum-weighted charges of the two hemispheres is believed to be brought about by the requirement that the total charge in the event is zero, that both hemispheres must consist only of color singlets, and also because 


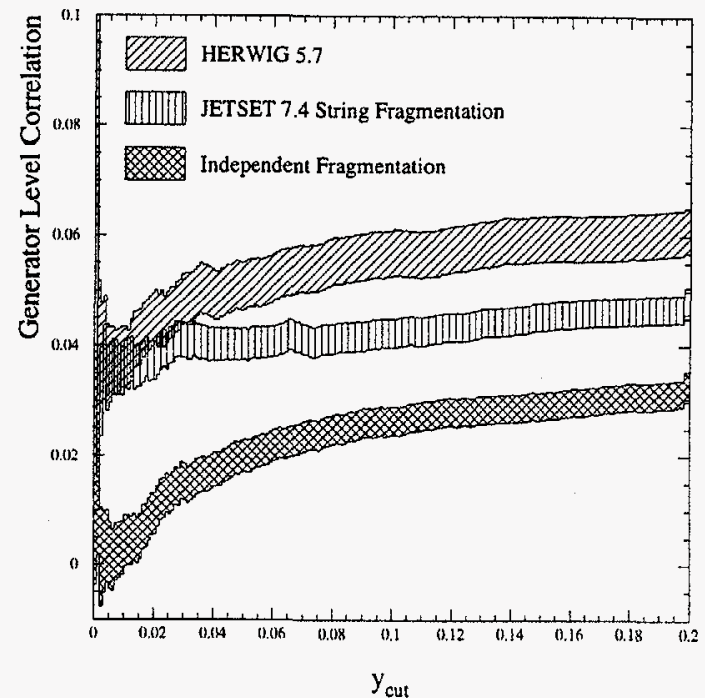

Figure 7.9: Comparison of generator-level correlations in the JETSET 7.4 string fragmentation model, JETSET's independent fragmentation option, and HERWIG 5.7. The correlations are shown for two-jet events where jets are found using the JADE algorithm [148], as a function of the jet resolution parameter $y_{\text {cut }}$. The widths of the shaded bands indicate the statistical error on the Monte Carlo simulation. The last bin to the right is the inclusive sample of all hadronic Monte Carlo decays.

some tracks may migrate from one hemisphere to the other, especially in events with hard gluon radiation.

The systematic error from this correlation was estimated by comparing the correlation parameter $\lambda$ for three different generators: JETSET 7.4 with parton shower and string fragmentation, JETSET 7.4 with the independent fragmentation option selected, and HERWIG 5.7 with default cluster fragmentation. The generators were allowed to decay unstable products, and a simple model of detector acceptance and analysis cuts was applied.

Figure 7.9 shows the generator-level correlations for two-jet events for the two
Table 7.4: Differences between the hemisphere correlations and their effects on the measured value of $A_{b}$. The fully-simulated JETSET string model was used in the analysis and is the basis of comparison for the other models. Monte Carlo statistical errors on the estimations of $\lambda$ are not propagated because the systematic variations are much larger.

\begin{tabular}{|lccc|}
\hline Model & Generator-Level $\lambda$ & Simulated $\lambda$ & $\Delta A_{b} / A_{b}$ \\
\hline JETSET 7.4 String Frag. & $(4.82 \pm 0.25) \%$ & $2.85 \%$ & - \\
JETSET 7.4 Indep't Frag. & $(3.30 \pm 0.25) \%$ & $1.96 \%$ & $-3.3 \%$ \\
HERWIG 5.7 & $(6.10 \pm 0.41) \%$ & $3.62 \%$ & $+3.2 \%$ \\
\hline
\end{tabular}

generators as a function of $y_{c u t}$, using the JADE algorithm on all stable generated particles. It is expected that the more two-jet like an event is, the less correlated it will be, as the task of conserving charge at the last quark popping will have lower momentum relative to the very fast reaction products in the two jets. For a threejet event, there is a more detailed charge conservation between the jets. Even the independent-fragmentation model shows a sizeable correlation at large values of $y_{c u t}$.

The relative difference between the correlations of JETSET's string fragmentation model and JETSET's independent fragmentation model was then taken as an estimate of the correlation uncertainty - the discrepancy with HERWIG's correlation was found to be smaller. The sensitivity of the measured $A_{b}$ to $\lambda$, holding $\sigma_{s u m}$ and $\sigma_{\left|Q_{\text {diff } f}\right|}$ fixed, was found to be

$$
\frac{\partial A_{6}}{\partial \lambda}=3.34
$$

The resulting relative systematic error from hemisphere correlations is a relative $3.3 \%$.

\subsubsection{Light Flavor Subtraction}

Accounting for light-flavor contamination when calculating $\sigma_{s u m, b}$ and $\sigma_{\left|Q_{\text {diff }}\right|, b}$ using Equations 7.30 and 7.31 changes the resulting $\alpha_{b}$ by very little. The systematic error is conservatively taken to be the difference between using an $\alpha$ corrected for lightflavor contamination and using an $\alpha$ that omits this correction. The net effect on the measured value of $A_{b}$ is a relative $0.2 \%$. 


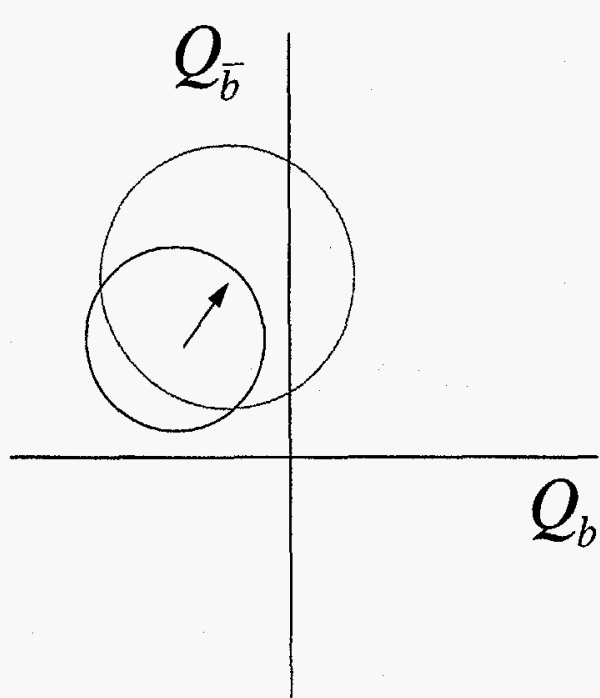

Figure 7.10: Effect of detector material on the joint probability distribution of $Q_{b}$ and $Q_{\bar{b}}$. The distributions widen, and an average positive offset is given to both $Q_{b}$ and $Q_{\bar{b}}$

\subsubsection{Detector material}

The extra positively-charged tracks introduced by nuclear scattering with the detector material have two effects on the ability of the momentum-weighted charge measurement to extract $A_{b}$. The first effect is to add some positive charge to the $b$ and $\bar{b}$ hemispheres on average, and the second is to broaden the $Q_{b}$ and $Q_{\bar{b}}$ distributions.

The second effect is calibrated by the sum and difference widths, as it makes the joint Gaussian distribution of $Q_{b}$ and $Q_{\bar{b}}$ larger in both directions by the same amount. The first effect is a genuine bias to the extraction of the analyzing power. It shifts the position of the mean of the joint distribution, increasing the $\sigma_{\text {sum }}$ without affecting $\sigma_{\left|Q_{\text {diff }}\right|}$. Fortunately, this bias can be measured by finding $\left\langle Q_{\text {sum }}\right\rangle$ in the data. This has the effect of modifying the sum width:

$$
\sigma_{\text {sum }} \rightarrow \sqrt{\sigma_{\text {sum }}^{2}+\left\langle Q_{\text {sum }}\right\rangle^{2}}
$$

The measured value of $\left\langle Q_{\text {sum }}\right\rangle$ is $0.088 \pm 0.034$. The systematic error is taken to be the difference in the measured $A_{b}$ with $\sigma_{\text {sum }}$ corrected for this effect, and without, and amounts to a relative $0.1 \%$ error.

\subsubsection{Light-flavor coupling asymmetries}

The sensitivity of the measured $A_{b}$ to the assumptions of the values of $A_{c}, A_{u d s}$, and $A_{\varepsilon}$ was estimated by re-analyzing the data with different values to compute the derivatives:

$$
\begin{aligned}
& \frac{\partial A_{b}^{\text {meas }}}{\partial A_{c}^{\text {assumed }}}=0.091, \\
& \frac{\partial A_{b}^{\text {meas }}}{\partial A_{b c k g}^{\text {assumed }}}=0.01,
\end{aligned}
$$

and

$$
\frac{\partial A_{b}^{\text {meas }}}{\partial A_{\epsilon}^{\text {assumed }}}=0.001
$$

A feature of the likelihood function is that the assumptions for $A_{b c k g}$ and $\alpha_{b c k g}$ : which parameterize the $p^{\text {"correct",bckg }}\left(\left|Q_{\text {diff }}\right|\right)$ of Equation 7.4, really constitute just one assumption. The strategy chosen here is to hold fixed a maximal $\alpha_{b c k g}$, and then to vary $A_{b c k g}$ within large limits. The $\alpha_{b c k g}$ was chosen to be that estimated from Monte Carlo $Z^{0} \rightarrow u \bar{u}$ events, the largest $\alpha$ of the five flavors. Then $A_{b c k g}$ was varied between +0.5 and -0.5 ; the central value chosen for the analysis was zero. The value of $A_{b c k g}$ is expected to be small because of the presence of the three light quark flavors with partially canceling charge asymmetries. The variation in the measured $A_{b}$ is quoted as a $0.6 \%$ relative systematic error.

The value of $A_{c}$ was fixed to the Standard Model value and its allowed range was large enough to accommodate conservatively the full range of the LEP average of $0.65 \pm 0.05$. The dependence of the answer on $A_{c}$ has been discussed in Section 7.2, although since it appears in the likelihood function, it constitutes model dependence on the answer. Its value was chosen within the range $A_{e}=0.1506 \pm 0.0028$, from 


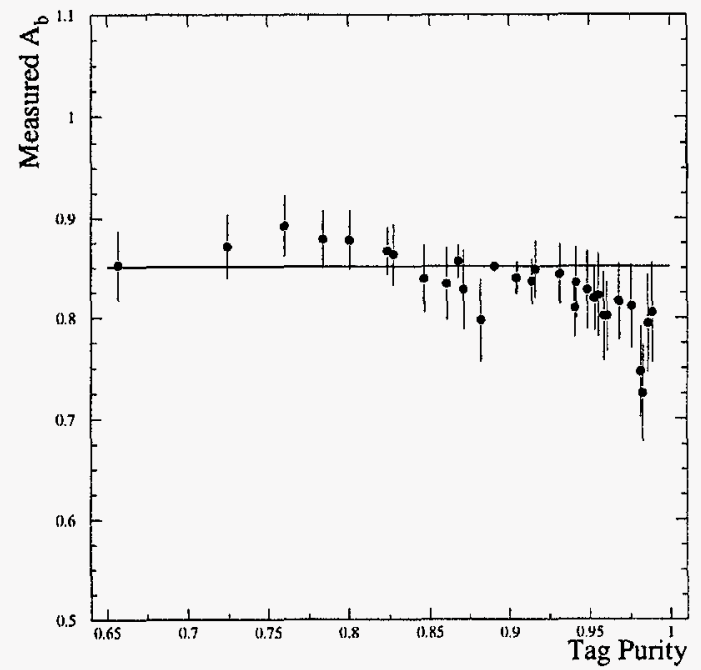

Figure 7.11: Tag dependence of the maximum-likelihood $A_{b}$ measurement, for tags ranging from 2 tracks at $2 \sigma$ to 4 tracks at $9 \sigma$. Correlated statistical and systematic errors have been removed, taking the 3 tracks at $3 \sigma$ point (with horizontal line) as a reference.

Table 2.1, which corresponds to the combined measurement of the LEP lepton asymmetries and the SLD $A_{L R}$ measurement.

\subsubsection{Tag purity}

Contributions to the systematic error arising from the modeling of the 2-D impact parameter tag are estimated with the aid of Table 6.1. Estimating the effect on the maximum-likelihood fit of $A_{b}$ is done by adding extra charm contamination to the estimation of the tag composition and re-fitting for $A_{b}$. The addition of uds events has half of the effect on the answer, owing to its smaller asymmetry. Furthermore, it is expected that the dominant contamination to the $B$-tagged sample is $Z^{0} \rightarrow c \bar{c}$ events, as the error from beam-position tails in Table 6.1 is not a leading contribution.
The same cross-check used for the binned asymmetry fit of checking the answer as a function of the hardness of the tag, described in Section 6.4.2, has been repeated for this analysis. The dependence, shown for tags ranging in purity from 2 tracks at $2 \sigma$ to 4 tracks at $9 \sigma$, are shown in Figure 7.11.

\subsubsection{QCD correction}

The systematic error arising from injecting a model of hard gluon radiation has several components. There is no second-order calculation of the QCD correction using massive quarks. Instead, first-order calculations, which incorporate quark mass, are used in the analysis, and the relative size of the second-order term, compared to the massless first-order correction, are taken as a systematic error. In addition, the uncertainty in the value of $\alpha_{s}$ introduces an uncertainty in this correction. The error is taken to be the quadrature sum of the estimated mistake by omitting the second-order terms and twice the current theoretical uncertainty in the value of $\alpha_{s}=0.118 \pm 0.010$ (theory).

\subsection{Normalized Momentum-Weighted Charge}

The approach followed by most competing analyses is to normalize the momentumweighted charge instead of using the unnormalized sum of Equation 6.2. The normalized momentum-weighted charge is given by

$$
Q_{\text {norm }}=\frac{-\sum_{\text {tracks }} q_{i}\left|\vec{p}_{i} \cdot \vec{t}\right|^{\kappa} \operatorname{sgn}\left(\vec{p}_{i} \cdot \vec{t}\right)}{\sum_{\text {tracks }}\left|\vec{p}_{i} \cdot \vec{t}\right|^{\kappa}}
$$

This quantity can be computed in the two hemispheres of each event and the selfcalibrated analysis of this chapter repeated. It is important to normalize the hemspheres separately before forming $Q_{\text {sum }}$ and $Q_{\text {diff }}$, in order to minimize the hemisphere correlation. The details of this analysis are given in Table 7.6. The fit to the data has slightly larger errors from statistics and $\alpha$ statistics than does the unnormalized fit, although the result is in excellent agreement. The normalized fit to the Monte Carlo accurately reproduces the input value of 0.9357 . The Monte Carlo distribution of $Q_{\text {norm }}$ fits slightly less well to a Gaussian than does the unnormalized $Q$ - the 
Table 7.5: Systematic error summary for the self-calibrated maximum-likelihood measurement of $A_{b}$

\begin{tabular}{|lcc|}
\hline Error Source & Variation & $\delta A_{b} / A_{b}$ \\
\hline Self-Calibration & & \\
\hline$\alpha_{b}$ Statistics & $1 \sigma$ & $3.4 \%$ \\
$p\left(Q_{b}\right)$ Shape & Triangular, other shapes & $1.0 \%$ \\
$\cos \theta$ shape of $\alpha_{b}$ & $\left\langle n_{c h}\right\rangle$ and $\left\langle Q_{\text {diff } f}\right\rangle$ shapes & $1.5 \%$ \\
Hemisphere Correlation & JETSET,HERWIG,Indep't & $3.3 \%$ \\
Light Flavor Subtraction & $100 \%$ & $0.2 \%$ \\
Detector Material & $100 \%$ & $0.1 \%$ \\
\hline Analysis & & \\
\hline$A_{c}$ & $0.67 \pm 0.07$ & $1.0 \%$ \\
$A_{u d s}$ & $0.0 \pm 0.50$ & $0.6 \%$ \\
$A_{c}$ & $0.1506 \pm 0.0028$ & $\ll 0.1 \%$ \\
$\alpha_{c}$ & Mostly $x_{D} \pm 5 \%$ & $0.2 \%$ \\
Tag Composition & See Table 6.1 & $2.6 \%$ \\
$P_{e}$ & $0.8 \%$ & $0.8 \%$ \\
QCD & $\alpha_{s} \pm 0.02,2^{n d}$ order terms & $0.9 \%$ \\
\hline Total & & $5.9 \%$ \\
\hline
\end{tabular}

Monte Carlo $p^{\text {correct,b }}\left(\left|Q_{\text {norm }}\right|\right)$ is shown in Figure 7.12, which may be compared with Figure 7.2. The normalized momentum-weighted charge is therefore not used in this analysis.

\subsection{Summary}

This chapter has presented a measurement of $A_{b}$ with a self-calibrated maximumlikelihood analysis. The calibration of the effectiveness of the technique was extracted from the widths of two distributions - the sum and the difference of the
Table 7.6: Details of the normalized momentum-weighted charge maximum-likelihood fit for $A_{b}$

\begin{tabular}{|lcc|}
\hline & Data & MC \\
\hline$\sigma_{\text {sum }}$ & $0.3289 \pm 0.0020$ & $0.3339 \pm 0.00089$ \\
$\sigma_{\left|Q_{\text {diff }}\right|}$ & $0.3745 \pm 0.0024$ & $0.37881 \pm 0.00101$ \\
$\alpha_{b}$ & $2.80 \pm 0.14$ & $2.70 \pm 0.06$ \\
$\lambda$ & Same as MC & $2.51 \%$ \\
Measured $A_{b}$ & $0.8497 \pm 0.048$ (stat.) & $0.944 \pm 0.020$ (stat.) \\
$\delta A_{b} / A_{b}$ from $\alpha$ statistics & $3.7 \%$ & $1.8 \%$ \\
\hline
\end{tabular}

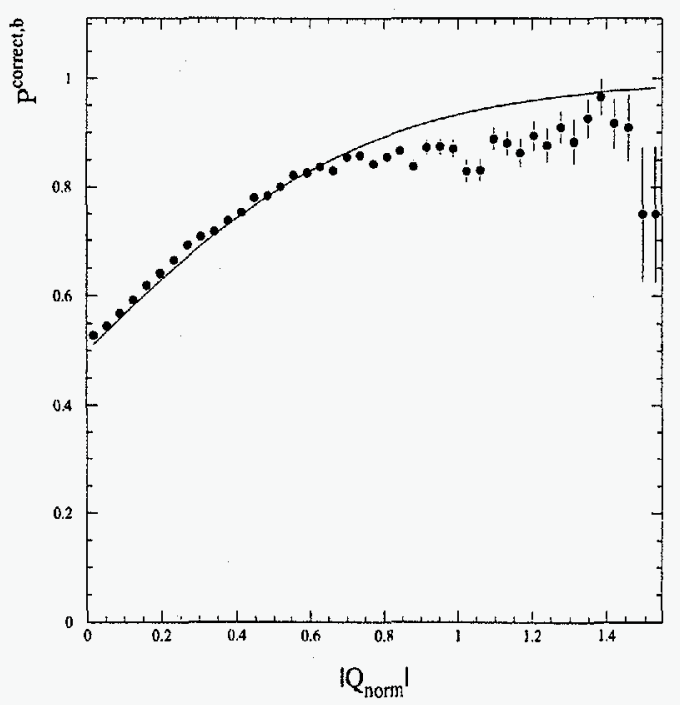

Figure 7.12: Correct-sign probability estimate for normalized momentum-weighted charge. The better fit of Figure 7.2 encourages the use of the unnormalized momentum-weighted charge. 
two hemisphere's momentum-weighted charges. Residual model dependencies come from interhemisphere correlation, the composition of the $b$-tagged sample, and $A_{c}$. Additional systematic error comes from the statistical error on the calibration. The measured value is

$$
A_{b}=0.848 \pm 0.046 \text { (stat.) } \pm 0.050 \text { (syst.). }
$$

\section{Chapter 8}

\section{Global Fit to $Z b b$ Vertex Parameters}

The contribution SLD makes to the knowledge of the coupling of the $b$ quark to the $Z^{0}$ is substantial. Takeuchi, Grant, and Rosner recognized this [170] and proposed a model-independent parameterization of $Z b b$ vertex couplings similar to the $S, T$. and $U$ parameters of Peskin and Takeuchi [60][61], which describe propagator corrections. Alternative parameterizations of the vertex couplings exist [173], and the parameters are easily re-expressed in terms of those of Takeuchi, Grant, and Rosner.

\subsection{Independent Parameters}

There are only two independent parameters describing the coupling of any fermion to the $Z^{\circ}$, and different combinations have different physical interpretations *. There are the vector and axial-vector couplings $v$ and $a$; there are the left-handed and righthanded couplings $c_{R}$ and $c_{L}$; and there are the overall coupling strength and parityviolating asymmetry, given by the observables $R_{f}$ and $A_{f}$. Because the coupling of left-handed $b$ quarks to the $Z^{0}$ is so much stronger than the coupling of righthanded $b$ quarks, a measurement of the overall coupling strength is most sensitive

*Some couplings are forced to zero by symmetry principles, such as the right-handed couplings to neutrinos. 
to corrections to the left-handed coupling, while a measurement of the asymmetry is most sensitive to corrections to the right-handed coupling. The current experimental state places a much looser constraint on the right-handed coupling of the $b$ quark to the $Z^{0}$ than it does the left-handed coupling. To extract the best estimate of the left and right-handed couplings, a global fit to available data sensitive to these quantities is required.

A complication arises in that the asymmetry $A_{b}$ depends slightly on $\sin ^{2} \theta_{W}$. Furthermore, the measurements of $A_{F B}^{b}$ from LEP are indirect measurements of $A_{b}$. Those measurements are significantly dependent on $\sin ^{2} \theta_{W}$ through the asymmetry in the initial state electron couplings to the $Z^{0}$.

To parameterize conveniently the dependence on the left- and right-handed $b$ couplings and also $\sin ^{2} \theta_{W}$, Takeuchi, Grant, and Rosner proposed the following scheme to perform a global fit to available observables:

$$
\begin{aligned}
\sigma_{\text {had }}^{0} & =\left[\sigma_{\text {had }}^{0}\right]_{S M}\left(1+0.11 \delta s^{2}+0.41 \xi_{b}\right), \\
R_{Z} \equiv \Gamma_{\text {had }} / \Gamma_{l^{+} l^{-}} & =\left[R_{Z}\right]_{S M}\left(1-0.85 \delta s^{2}-1.02 \xi_{b}\right), \\
R_{b} \equiv \Gamma_{b \bar{b}} / \Gamma_{\text {had }} & =\left[R_{b}\right]_{S M}\left(1+0.18 \delta s^{2}-3.63 \xi_{b}\right), \\
R_{c} \equiv \Gamma_{c \bar{c}} & =\left[R_{c}\right]_{S M}\left(1-0.35 \delta s^{2}+1.02 \xi_{b}\right), \\
A_{b} & =\left[A_{b}\right]_{S M}\left(1-0.68 \delta s^{2}-1.76 \zeta_{b}\right), \\
A_{F B}^{b}=\frac{3}{4} A_{e} A_{b} & =\left[A_{F B}^{b}\right]_{S M}\left(1-55.7 \delta s^{2}-1.76 \zeta_{b}\right) .
\end{aligned}
$$

The variables chosen are

$$
\begin{aligned}
\delta s^{2} & =\sin ^{2} \theta_{W}^{e f f}-\left[\sin ^{2} \theta_{W}^{e f f}\right]_{S M}, \\
\zeta_{b} & =\left(\sin \phi_{b}\right) \delta c_{L}^{b}+\left(\cos \phi_{b}\right) \delta c_{R}^{b}, \\
\xi_{b} & =\left(\cos \phi_{b}\right) \delta c_{L}^{b}-\left(\sin \phi_{b}\right) \delta c_{R}^{b},
\end{aligned}
$$

where

$$
\phi_{b}=\tan ^{-1}\left|c_{R}^{b} / c_{L}^{b}\right| \approx 0.181 .
$$

This linear combination of the chiral couplings $c_{L, R}^{b}$, defined in Chapter 1 , is convenient because it forms a basis in which the measurements of production rates and ratios are orthogonal to the measurements of asymmetries.
Table 8.1: Measured observables contributing to the global fit to $Z b b$ vertex parameters. The measurements are preliminary results reported at the 1995 Summer Europhysics Conference in Brussels [64]. The SLD measurement of $A_{b}$ comes from this thesis, the semileptonic $b$ asymmetry measurement [47], and the kaon measurement [50]. The value of $\sin ^{2} \theta_{W}$ from LEP is averaged only over lepton channel measurements to avoid circularity. The SM predictions were obtained using ZFITTER [51], with $m_{\text {Higgs }}=300 \mathrm{GeV} / \mathrm{c}^{2}, m_{\imath}=$ $180 \mathrm{GeV} / \mathrm{c}^{2}$, and $\alpha_{s}=0.117$, and $\alpha_{E M}=1 / 128.96[171]$.

\begin{tabular}{|lcc|}
\hline Measurement & Value & SM Prediction \\
\hline $\sin ^{2} \theta_{W}$ (SLD) & $0.23049 \pm 0.0050$ & 0.23163 \\
$\sin ^{2} \theta_{W}$ (LEP) & $0.23160 \pm 0.0049$ & 0.23163 \\
$A_{b}$ (SLD) & $0.859 \pm 0.053$ & 0.9346 \\
$A_{F B}^{b}$ (LEP) & $0.0997 \pm 0.0031$ & 0.10247 \\
$\sigma_{\text {had }}^{0}$ (LEP) & $41.488 \pm 0.078 \mathrm{nb}$ & 41.485 \\
$R_{Z}$ (LEP) & $20.788 \pm 0.039$ & 20.730 \\
$R_{b}$ (LEP, SLD) & $0.2219 \pm 0.0017$ & 0.21552 \\
$R_{c}$ (LEP) & $0.1540 \pm 0.0074$ & 0.1723 \\
\hline
\end{tabular}

\subsection{Fit Inputs}

The best available data at the time of this writing comes from presentations at the 1995 Europhysics conference [64][65], and a summary of available observables is given in Table 8.1. In addition to the observables, Standard Model values must be calculated for these observables. The most poorly constrained parameters important to the predictions of these quantities are the values of $m_{t}, m_{\text {Higgs }}$, and $\alpha_{s}\left(m_{Z}\right)$. The values chosen for this analysis are $m_{t}=180 \mathrm{GeV}, m_{\text {Higgs }}=300 \mathrm{GeV}$, and $\alpha_{s}\left(m_{Z}\right)=0.120 \pm$ 0.006 , as determined from event shapes, jet rates, and energy correlations. A fit value of $\alpha_{s}$ using lineshape data would introduce correlations with the $\sigma_{\text {had }}^{\mathrm{o}}$ and $R_{Z}$ inputs to the fit. In what will be shown below, the range of predictions of the Standard Model for a range of $m_{t}$ and $m_{\text {Higgs }}$ will be shown. 


\subsection{Constraints on Parameters}

A global fit to the available data taking into account experimental correlations has been performed by Bruce Schumm [172], and the results are summarized here. It can be seen that the rate parameter $\xi_{b}$ differs from its expected value in excess of $3 \sigma$, while the asymmetry parameter $\zeta_{b}$ differs by $2 \sigma$. These are shown graphically in Figures 8.1 and 8.2 .

$$
\begin{aligned}
& \delta s^{2}=-0.00055 \pm 0.00036 \\
& \delta \xi_{b}=-0.0047 \pm 0.0015 \\
& \delta \zeta_{b}=0.032 \pm 0.018
\end{aligned}
$$

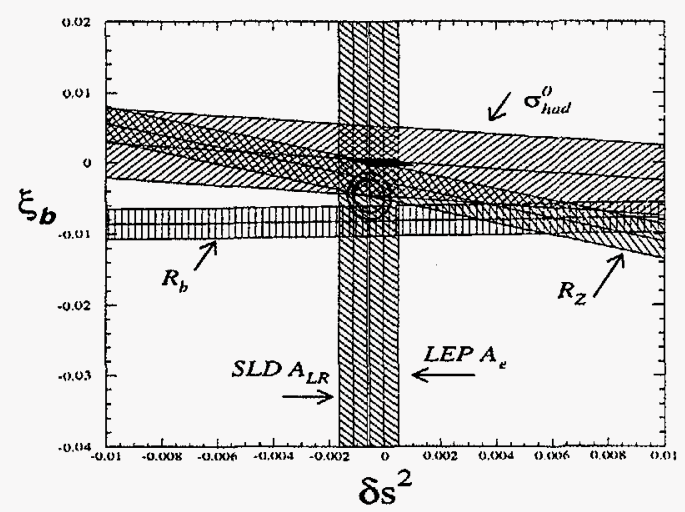

Figure 8.1: Combined constraints on the $b$ rate-like parameter $\xi_{b}$ shown with $\delta s^{2}$. The band for $R_{c}$ falls below the figure with a large error bar. The ellipses are the $68 \%$ and $95 \%$ confidence-level fits to the bands in the figure, and the parallelogram is the range of Standard Model predictions, with $m_{t}=192$ on the top side, $m_{t}=168$ on the bottom, $m_{H}=60$ on the left side, and $m_{H}=1000$ on the right. This diagram displays graphically: the discrepancy of the $R_{b}$ measurements with the Standard prediction, perhaps suggesting new phenomena which modify the $Z b b$ coupling.

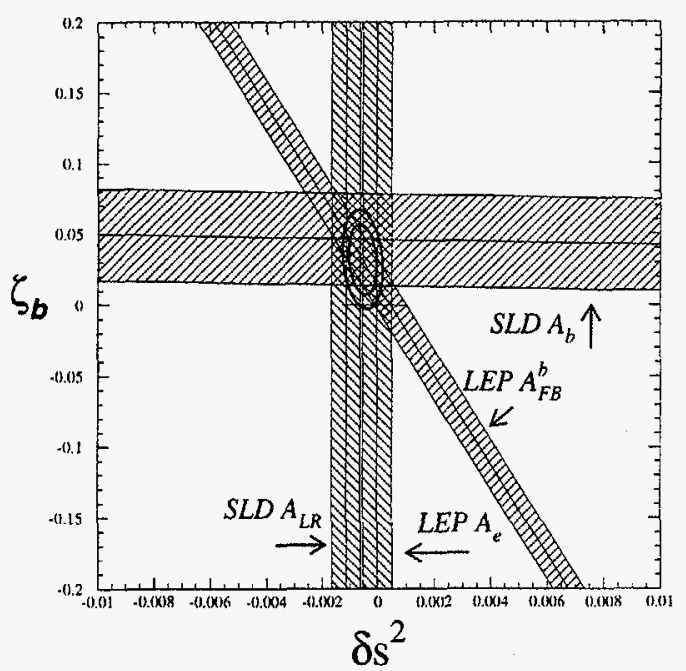

Figure 8.2: Combined constraints on the $b$ asymmetry-like parameter $\zeta_{b}$ shown with $\delta s^{2}$, The ellipses are the $68 \%$ and $95 \%$ confidence-level fits to the bands in the figure, and the horizontal line is the Standard Model allowing $m_{t}$ to vary between 168 and $192 \mathrm{GeV} / \mathrm{c}^{2}$, and allowing $m_{H}$ to vary between 60 and $1000 \mathrm{GeV} / \mathrm{c}^{2}$. Light Higgs and heavy top correspond to the leftmost Standard Model points. The Standard Model's prediction for $\zeta_{b}$, probed most directly by measurements of $A_{b}$, is rather rigid under variations of its parameters. 


\section{Chapter 9}

\section{Summary and Outlook}

\subsection{Results and Comparison}

This thesis has presented two direct measurements of $A_{b}$, the parity-violating asymmetry of the coupling of $b$ quarks to the $Z^{0}$ boson. The first technique lifetime-tags $Z^{0} \rightarrow b \bar{b}$ events and fits the data binned to form the left-right forward-backward asymmetry where the thrust axis has been signed with the event momentum-weighted track charge. The SLD Monte Carlo, incorporating JETSET 7.4, the CLEO B meson decay model, and GEANT detector simulation, was used to estimate the effectiveness of the momentum-weighted track charge assignment and to correct for efficiency, acceptance, and resolution effects, as well as the effects of initial-state QED radiation and final-state gluon radiation. This measurement yields

$$
A_{b}=0.828 \pm 0.054 \text { (stat.) } \pm 0.070 \text { (syst.). }
$$

The systematic error arises almost entirely from the use of the Monte Carlo to estimate the performance of the momentum-weighted charge-signing procedure and the composition of the tag.

The second technique, a self-calibrated maximum-likelihood method, was developed in order to reduce the total error on the measurement as an increasingly large sample of hadronic $Z^{0}$ decays becomes available. It minimizes the statistical error on the measurement by taking advantage of the magnitude of the momentum-weighted charge of each event, as well as its sign. Each event receives its own weight in the likelihood sum depending on how reliable the estimation of the sign is.

The systematic error in the maximum-likelihood technique has been reduced by introducing a calibration procedure which uses the data almost exclusively to estimate how often the momentum-weighted track charge signs $Z^{0} \rightarrow b \bar{b}$ events properly. This technique uses the momentum-weighted charge in the two hemispheres of each event to determine the correct-sign probability. Residual model dependence comes from estimating the correlation of the momentum-weighted charge in the two hemispheres of an event, estimating the light-flavor contamination to the tag and its asymmetry, and the modeling of QCD final-state radiation. The resulting measurement of this analysis is

$$
A_{b}=0.848 \pm 0.046 \text { (stat.) } \pm 0.050 \text { (syst.): }
$$

While these measurements and the Standard Model prediction of $A_{b}=0.936$ are consistent, higher-precision measurements in the future may begin to show a deviation.

SLD is in a unique position to measure $A_{b}$ because experiments with unpolarized beams may only measure the combination $A_{F B}^{b}=\frac{3}{4} A_{\varepsilon} A_{b}$, while SLC's polarized electron beam allows a factorization of the component asymmetries. If one takes the average $A_{F B}^{b}$ from the four LEP experiments and divides it by $A_{\varepsilon}=0.1506 \pm 0.0028$ as determined from lepton forward-backward asymmetries, then the LEP measurement of $A_{b}$ is [174]

$$
A_{b}=0.883 \pm 0.032
$$

where lepton and momentum-weighted track charge measurements from each of the four experiments have been combined*. It can be seen in Chapter 2 that the measurement presented in this thesis is one of the the best measurements ${ }^{\dagger}$ of $A_{6}$ at present. and uses only $150,000 Z^{0}$ decays.

"L3 did not contribute a momentum-weighted track charge measurement.
TOnly the ALEPH semileptonic $A_{F B}^{b}$ has a smaller total error. It is one of the outputs of a fit for $A_{F B}^{c}, \chi_{B}$, and $\operatorname{Br}(b \rightarrow c \rightarrow l)$, and so errors on these other outputs are not included in the error on $A F B$. 


\subsection{Track Charge Prospects}

Even with the calibration from the data, the systematic error on the measurement of Chapter 7 is larger than the statistical error. If the necessary precision of $\sim 1 \%$ is to be achieved, further work must be done on the systematic error. The statistical error on $\alpha_{b}$ will scale with $1 / \sqrt{N}$, where $N$ is the total event count, and the error on the tag composition may be reduced by using tighter event tags or hemisphere double tags. The correlation error appears to be the most difficult to understand.

One approach to reduce the error from uncertainties in the hemisphere correlation is to measure it from the data. The procedure of finding the correlation of the unsigned hemisphere charges was described in Section 7.3.3, although its statistical porver is limited by available data. A search for more effective measurements of the correlation should be made.

A strategy for improving the momentum-weighted track charge technique is to try different weighting functions rather than raising the momentum to a fixed power. Care must be taken, however, not to choose a function merely because it maximizes the observed asymmetry in the data, as that can introduce measurement bias.

Other information can be used to help discriminate $b$ from $\bar{b}$. The VXD offers precise measurements of track impact parameters to the IP. Extra weight could be given to those tracks with significant impact parameters, or to identified kaons, electrons. or muons.

Progress is being made to improve the $b$-tag. A three-dimensional probability tag [147] can provide an event tag that has a $b$-purity of $\sim 95 \%$ at the same efficiency. as the tag used in this thesis. This can reduce the systematic error contributions from the tag composition by a factor of two, and reduce the statistical error at the same time. The raw statistical error is expected to scale inversely with $\sqrt{\epsilon \Pi}$, where $\epsilon$ is the tagging efficiency and $\Pi$ is the purity, a result which can be obtained from Equation 6.5.

\subsection{New Techniques}

The main shortcoming of momentum-weighted track charge is its analyzing power of $\sim 36 \%$. The statistical power of the technique scales as $1 /\left(A P \cdot P_{e} \cdot \sqrt{N}\right)$, and the large gains can be made by increasing $A P$. "Exclusive" techniques have higher $A P$ but also a reduced $N$, because they must select a specific, and often very restricted, subsample of events that have a high average analyzing power.

A measurement using charged kaons [50] has already been performed at SLD. Kaon tags can be used alone or in combination with vertexing to achieve a sample with a high-purity of correctly-signed $Z^{0} \rightarrow b \bar{b}$ events.

Measurements of $A_{c}$ using $D^{*}$ mesons have been recently performed at SLD [175][176] and elsewhere [177][178][179]. These measurements typically use a hard momentum cut on the $D^{*}$ in order to reject cascade $B \rightarrow D^{*}$ decays. The SLD analysis also uses displaced vertices and the precise beam spot to reject cascaded decays because the $B$ and $D$ flight paths are not collinear. These analyses can open their requirements to include $B \rightarrow D^{*}$ and fit for $A_{b}$ as well as $A_{c}$. The analyzing power of the $D^{*}$ is very high, although $B$ mixing has to be accounted for.

Recently an effort has been invested in finding topological vertices using the VXD [180]. It has been used successfully to separate enriched samples of $B^{0}$ and $B^{ \pm}$mesons for a measurement of the lifetime ratio $\tau_{B^{ \pm}} / \tau_{B^{0}}$. A pure sample of $B^{+}$mesons could identify every event's decay axis sign correctly in an asymmetry analysis. The only tasks are to raise the efficiency sufficiently, and to measure the purity.

All of these techniques will benefit from using SLD's upgraded vertex detector, VXD3 [181]. The upgraded detector will increase the lever arm of the measurement by expanding the outer layers while the inner layer stays fixed. Each layer will have larger CCD's that are half as thick as those of the current detector. The expected measurement errors on impact parameters and vertex locations are about half those of the current vertex detector over the entire momentum range. This new detector will greatly improve the ability to assign tracks to vertices unambiguously, which will aid in determining the charge of long-lived heavy hadrons. It will also allow more efficient samples of $D^{*}$ mesons to be made, because tightening the requirements on 
the vertex will allow other selection cuts to be relaxed.

\subsection{Calibrating New Techniques}

The analyzing power of the new techniques is not expected to be exactly unity mixing, double-charm decay, and other physics effects, as well as detector response, will reduce the analyzing power. The distributions of discriminating variables for these new techniques is often discrete, so the Gaussian approximation of Chapter 7 will not be useful.

The analyzing power for a technique with a discrete-valued discriminant can still be calibrated using the data from the two event hemispheres, though. If $P_{b}(n)$ is the probability of observing $n$ in the $b$ hemisphere, and

$$
P_{\bar{b}}(n)=P_{b}(-n)
$$

is the probability of observing $n$ in the $\bar{b}$ hemisphere, assuming $C P$ symmetry. If we define hemisphere sums and differences as in Chapter 7 ,

$$
\begin{aligned}
& n_{\text {sum }}=n_{b}+n_{\bar{b}}, \text { and } \\
& n_{\text {diff }}=n_{b}-n_{\bar{b}},
\end{aligned}
$$

then the probabilities $P_{s}\left(n_{\text {sum }}\right)$ and $P_{d}\left(\left|n_{d i f f}\right|\right)$ are directly measurable from the data. If $C P$ symmetry holds, then the condition

$$
P_{s}\left(n_{s u m}\right)=P_{s}\left(-n_{s u m}\right)
$$

can be used to improve the statistical power.

This leads us to an over-constrained system of quadratic equations:

$$
\begin{aligned}
& P_{s}\left(\left|n_{\text {sum }}\right|\right)=\sum_{\left|n_{b}+n_{\bar{b}}\right|=\left|n_{s u m}\right|} P_{b}\left(n_{b}\right) P_{b}\left(-n_{\bar{b}}\right), \quad \text { and } \\
& P_{d}\left(\left|n_{\text {diff }}\right|\right)=\sum_{\left|n_{b}-n_{\bar{b}}\right|=\left|n_{\text {diff }}\right|} P_{b}\left(n_{b}\right) P_{b}\left(-n_{\bar{b}}\right) .
\end{aligned}
$$

If one is interested in solving for $2 N+1$ values of $P_{b}(n)$, where $n \in[-N, N]$, then one has $4 N+2$ equations above to constrain them, as $\left|n_{s u m, d i f f}\right| \in[0,2 N]$. In addition. there is the constraint that $P_{b}(n)$ be normalized.
This procedure makes no assumptions about the shape of $P_{b}(n)$, so virtually any combination of detector variables may be used without concern about modeling its distribution. It does assume that the hemispheres are uncorrelated, though, and it works best when the fraction of events with both hemispheres contributing information is high. Combination techniques with momentum-weighted track charge may be used to improve the efficiency to perform this calibration.

Techniques which provide continuous outputs from unknown distributions can also be calibrated with this procedure, if the measured observables are first binned. Understanding the underlying distributions, however, will always provide an advantage.

\subsection{Outlook}

SLC is scheduled to run until 1998 , by which time 500,000 hadronic $Z^{\circ}$ decays are expected to have been recorded. The LEP ring at CERN stopped running at the $Z^{0}$ in October, 1995, leaving SLD in a leading position in tests of the Electroweak Standard Model. The analysis presented in this thesis is one of the important tests that can be made at SLD and may, with improvements available with more data, reach the precision necessary to discriminate between extensions to the current models. 


\section{Appendix A}

\section{SLD Collaboration}

K. Abe, ${ }^{(29)}$ I. Abt, ${ }^{(14)}$ C.J. Ahn, ${ }^{(26)}$ T. Akagi, ${ }^{(27)}$ N.J. Allen, ${ }^{(4)}$ W.W. Ash, ${ }^{(27) \dagger}$ D. Aston, ${ }^{(27)}$ K.G. Baird, ${ }^{(25)}$ C. Baltay, ${ }^{(33)}$ H.R. Band, ${ }^{(32)}$ M.B. Barakat, ${ }^{(33)}$

G. Baranko, ${ }^{(10)}$ O. Bardon, ${ }^{(16)}$ T. Barklow, ${ }^{(27)}$ A.O. Bazarko, ${ }^{(11)}$ R. Ben-David, ${ }^{(33)}$ A.C. Benvenuti, ${ }^{(2)}$ T. Bienz, ${ }^{(27)}$ G.M. Bilei, ${ }^{(22)}$ D. Bisello, ${ }^{(21)}$ G. Blaylock, ${ }^{(7)}$ J.R. Bogart, ${ }^{(27)}$ T. Bolton, ${ }^{(11)}$ G.R. Bower, ${ }^{(27)}$ J.E. Brau, ${ }^{(20)}$ M. Breidenbach, ${ }^{(2 i)}$ W.M. Bugg, ${ }^{(28)}$ D. Burke, ${ }^{(27)}$ T.H. Burnett, ${ }^{(31)}$ P.N. Burrows, ${ }^{(16)}$ W. Busza ${ }^{(16)}$

A. Calcaterra, ${ }^{(13)}$ D.O. Caldwell, ${ }^{(6)}$ D. Calloway, ${ }^{(27)}$ B. Camanzi, ${ }^{(12)}$ M. Carpinelli, ${ }^{(23)}$ R. Cassell, ${ }^{(27)}$ R. Castaldi, ${ }^{(23)(a)}$ A. Castro, ${ }^{(21)}$ M. Cavalli-Sforza, ${ }^{(7)}$

E. Church, ${ }^{(31)}$ H.O. Cohn, ${ }^{(28)}$ J.A. Coller, ${ }^{(3)}$ V. Cook, ${ }^{(31)}$ R. Cotton, ${ }^{(4)}$

R.F. Cowan, ${ }^{(16)}$ D.G. Coyne, ${ }^{(7)}$ A. D'Oliveira, ${ }^{(8)}$ C.J.S. Damerell, ${ }^{(24)}$ M. Daoudi, ${ }^{(2 i)}$

R. De Sangro, ${ }^{(13)}$ P. De Simone, ${ }^{(13)}$ R. Dell'Orso, ${ }^{(23)}$ M. Dima, ${ }^{(9)}$ P.Y.C. Du, ${ }^{(28)}$

R. Dubois, ${ }^{(27)}$ B.I. Eisenstein, ${ }^{(14)}$ R. Elia, ${ }^{(27)}$ E. Etzion, ${ }^{(4)}$ D. Falciai, ${ }^{(22)}$

M.J. Fero, ${ }^{(16)}$ R. Frey, ${ }^{(20)}$ K. Furuno, ${ }^{(20)}$ T. Gillman, ${ }^{(24)}$ G. Gladding, ${ }^{(14)}$

S. Gonzalez, ${ }^{(16)}$ G.D. Hallewell, ${ }^{(27)}$ E.L. Hart, ${ }^{(28)}$ Y. Hasegawa, ${ }^{(29)}$ S. Hedges, ${ }^{(4)}$ S.S. Hertzbach, ${ }^{(17)}$ M.D. Hildreth, ${ }^{(27)}$ J. Huber, ${ }^{(20)}$ M.E. Huffer, ${ }^{(27)}$ E.W. Hughes, ${ }^{(2 \pi)}$

H. Hwang, ${ }^{(20)}$ Y. Iwasaki, ${ }^{(29)}$ D.J. Jackson, ${ }^{(24)}$ P. Jacques, ${ }^{(25)} \mathrm{J} . \mathrm{Jaros},{ }^{(27)}$

A.S. Johnson, ${ }^{(3)}$ J.R. Johnson, ${ }^{(32)}$ R.A. Johnson, ${ }^{(8)}$ T. Junk, ${ }^{(27)}$ R. Kajjkawa. ${ }^{(19)}$

M. Kalelkar, ${ }^{(25)}$ H. J. Kang, ${ }^{(26)}$ I. Karliner, ${ }^{(14)}$ H. Kawahara, ${ }^{(27)}$ H.W. Kendall, ${ }^{(16)}$

Y. Kim, ${ }^{(26)}$ M.E. King, ${ }^{(27)}$ R. King, ${ }^{(27)}$ R.R. Kofier, ${ }^{(17)}$ N.M. Krishna, ${ }^{(10)}$

R.S. Kroeger, ${ }^{(18)}$ J.F. Labs, ${ }^{(27)}$ M. Langston, ${ }^{(20)}$ A. Lath, ${ }^{(16)}$ J.A. Lauber, ${ }^{(10)}$
D.W.G. Leith, ${ }^{(27)}$ M.X. Liu, ${ }^{(33)}$ X. Liu, ${ }^{(7)}$ M. Loreti, ${ }^{(21)}$ A. Lu, ${ }^{(6)}$ H.L. Lynch, ${ }^{(27)}$ J. Ma, ${ }^{(31)}$ G. Mancinelli, ${ }^{(22)}$ S. Manly, ${ }^{(33)}$ G. Mantovani, ${ }^{(22)}$ T.W. Markiewicz, ${ }^{(27)}$

T. Maruyama, ${ }^{(27)}$ R. Massetti, ${ }^{(2)}$ H. Masuda, ${ }^{(27)}$ E. Mazzucato, ${ }^{(12)}$

A.K. McKemey, ${ }^{(4)}$ B.T. Meadows, ${ }^{(8)}$ R. Messner, ${ }^{(2 \tau)}$ P.M. Mockett ${ }^{(31)}$

K.C. Moffeit, ${ }^{(27)}$ B. Mours, ${ }^{(27)}$ G. Müller, ${ }^{(27)}$ D. Muller, ${ }^{(27)}$ T. Nagamine, ${ }^{(27)}$

U. Nauenberg, ${ }^{(10)}$ H. Neal, ${ }^{(27)}$ M. Nussbaum, ${ }^{(8)}$ Y. Ohnishi, ${ }^{(19)}$ L.S. Osborne, ${ }^{(16)}$

R.S. Panvini, ${ }^{(30)}$ H. Park, ${ }^{(20)}$ T.J. Pavel, ${ }^{(27)}$ I. Peruzzi, ${ }^{(13)(b)}$ M. Piccolo, ${ }^{(13)}$

L. Piemontese, ${ }^{(12)}$ E. Pieroni, ${ }^{(23)}$ K.T. Pitts, ${ }^{(20)}$ R.J. Plano, ${ }^{(25)}$ R. Prepost, ${ }^{(32)}$ C.Y. Prescott, ${ }^{(27)}$ G.D. Punkar, ${ }^{(27)}$ J. Quigley, ${ }^{(16)}$ B.N. Ratcliff, ${ }^{(27)}$ T.W. Reeves, ${ }^{(30)}$ J. Reidy, ${ }^{(18)}$ P.E. Rensing, ${ }^{(27)}$ L.S. Rochester, ${ }^{(27)}$ J.E. Rothberg, ${ }^{(31)}$ P.C. Rowson, ${ }^{(11)}$ J.J. Russell, ${ }^{(27)}$ O.H. Saxton, ${ }^{(27)}$ S.F. Schaffner, ${ }^{(27)}$ T. Schalk, ${ }^{(7)}$ R.H. Schindler, ${ }^{(27)}$

U. Schneekloth, ${ }^{(16)}$ B.A. Schumm, ${ }^{(15)}$ A. Seiden, ${ }^{(7)}$ S. Sen, ${ }^{(33)}$ V.V. Serbo, ${ }^{(32)}$

M.H. Shaevitz, ${ }^{(11)}$ J.T. Shank, ${ }^{(3)}$ G. Shapiro, ${ }^{(15)}$ S.L. Shapiro, ${ }^{(27)}$ D.J. Sherden, ${ }^{(27)}$ K.D. Shmakov, ${ }^{(28)}$ C. Simopoulos, ${ }^{(27)}$ N.B. Sinev, ${ }^{(20)}$ S.R. Smith, ${ }^{(27)}$ J.A. Snyder, ${ }^{(33)}$ P. Stamer, ${ }^{(25)}$ H. Steiner, ${ }^{(15)}$ R. Steiner, ${ }^{(1)}$ M.G. Strauss, ${ }^{(17)}$ D. Su, ${ }^{(2 \pi)}$

F. Suekane, ${ }^{(29)}$ A. Sugiyama, ${ }^{(19)}$ S. Suzuki, ${ }^{(19)}$ M. Swartz, ${ }^{(27)}$ A. Szumilo, ${ }^{(31)}$

T. Takahashi, ${ }^{(27)}$ F.E. Taylor, ${ }^{(16)}$ E. Torrence, ${ }^{(15)}$ J.D. Turk, ${ }^{(33)}$ T. Usher, ${ }^{(2 T)}$

J. Va'vra, ${ }^{(27)}$ C. Vannini, ${ }^{(23)}$ E. Vella, ${ }^{(27)}$ J.P. Venuti, ${ }^{\left({ }^{20}\right)}$ R. Verdier, ${ }^{(16)}$ P.G. Verdini, ${ }^{(23)}$ S.R. Wagner, ${ }^{(27)}$ A.P. Waite, ${ }^{(27)}$ S.J. Watts, ${ }^{(4)}$

A.W. Weidemann, ${ }^{(28)}$ E.R. Weiss, ${ }^{(31)}$ J.S. Whitaker, ${ }^{(3)}$ S.L. White, ${ }^{(28)}$

F.J. Wickens, ${ }^{(24)}$ D.A. Williams, ${ }^{(7)}$ D.C. Williams, ${ }^{(16)}$ S.H. Williams, ${ }^{(27)}$

S. Willocq, ${ }^{(33)}$ R.J. Wilson, ${ }^{(9)}$ W.J. Wisniewski, ${ }^{(5)}$ M. Woods, ${ }^{(27)}$ G.B. Word, ${ }^{(25)}$

J. Wyss, ${ }^{(21)}$ R.K. Yamamoto, ${ }^{(16)}$ J.M. Yamartino, ${ }^{(16)}$ X. Yang, ${ }^{(20)}$ S.J. Yellin, ${ }^{(6)}$

C.C. Young, ${ }^{(27)}$ H. Yuta, ${ }^{(29)}$ G. Zapalac, ${ }^{(32)}$ R.W. Zdarko, ${ }^{(27)}$ C. Zeitlin, ${ }^{(20)}$ Z. Zhang, ${ }^{(16)}$ and J. Zhou, ${ }^{(20)}$

(1) Adelphi University, Garden City, New York 11530

(2) INFN Sezione di Bologna, I-40126 Bologna, Italy

(3) Boston University, Boston, Massachusetts 02215

${ }^{(4)}$ Brunel University, Uxbridge, Middlesex UB8 3PH, United Kingdom 
(5) California Institute of Technology, Pasadena, California 91125

${ }^{(6)}$ University of California at Santa Barbara, Santa Barbara, California 93106

(7) University of California at Santa Cruz, Santa Cruz, California 9.5064

(8) University of Cincinnati, Cincinnati, Ohio 45221

(9) Colorado State University, Fort Collins, Colorado 80523

(10) University of Colorado, Boulder, Colorado 80309

(11) Columbia University, New York, New York 10027

${ }^{(12)}$ INFN Sezione di Ferrara and Università di Ferrara, I-44100 Ferrara, Italy

${ }^{(13)}$ INFN Lab. Nazionali di Frascati, I-00044 Frascati, Italy

(14) University of Illinois, Urbana, Illinois 61801

${ }^{(15)}$ Lawrence Berkeley Laboratory, University of California, Berkeley, California 94720

${ }^{(16)}$ Massachusetts Institute of Technology, Cambridge, Massachusetts 02139

(17) University of Massachusetts, Amherst, Massachusetts 01003

(18) University of Mississippi, University, Mississippi 38677

${ }^{(19)}$ Nagoya University, Chikusa-ku, Nagoya 464 Japan

${ }^{(20)}$ University of Oregon, Eugene, Oregon 97403

(21) INFN Sezione di Padova and Università di Padova, I-35100 Padova, Italy

${ }^{(22)}$ INFN Sezione di Perugia and Università di Perugia, I-06100 Perugia, Italy

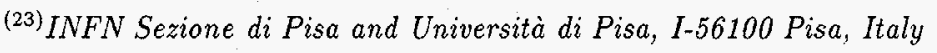

${ }^{(25)}$ Rutgers University, Piscataway, New Jersey 08855

${ }^{(24)}$ Rutherford Appleton Laboratory, Chilton, Didcot, Oxon OX11 $0 Q X$ United Kingdom

${ }^{(26)}$ Sogang University, Seoul, Korea

${ }^{(27)}$ Stanford Linear Accelerator Center, Stanford University, Stanford, California 94309

(28) University of Tennessee, Knoxville, Tennessee 37996 (29) Tohoku University, Sendai 980 Japan

(30) Vanderbilt University, Nashville, Tennessee 37235

(31) University of Washington, Seattle, Washington 98195

${ }^{(32)}$ University of Wisconsin, Madison, Wisconsin 53706
(33) Yale University, New Haven, Connecticut 06511

$$
\dagger_{\text {Deceased }}
$$

(a) Also at the Università di Genova

(b) Also at the Università di Perugia 


\section{Appendix B}

\section{Compton Polarimeter Online}

\section{Software}

The SLD Compton polarimeter measures the electron beam polarization by backscattering $532 \mathrm{~nm}$ laser photons off of the electron beam as it passes through the South Final Focus. Its operation is described in Chapter 3. In this Appendix the details of the online acquisition system specific to the Compton Polarimeter are given.

\section{B.1 Structure and Requirements}

Figure B.1 gives an overall road map to the acquisition, and provides an outline to the tasks performed. These tasks are:

- to acquire data from the hardware;

- to check the data for integrity;

- to calculate polarization and other useful quantities, such as signal and noise averages;

- to log the data to the SLD acquisition tape;

- to display the data online for monitoring and diagnostics;
- to distribute the calculated polarization to SLC and keep a history of it;

- to display the calculated polarization on the SLD status display, as well as detailed detector and data status;

- to perform routine scans of polarimeter detector parameters to control systematic errors;

- to provide remote control of the detector for diagnostics and measurements of systematic effects;

- to limit access to the control features to ensure that no hardware option is adjusted during data taking or during a special systematic test;

- to allow full monitoring ability, even for those users not directly controlling the data flow;

- to operate automatically with a minimum of intervention from shift personnel; and

- to be easy to upgrade when the Compton hardware is modified.

These tasks are similar to those faced by most of the online acquisition systems on SLD, and therefore the acquisition has a similar structure. The model used is a distributed client-server structure, in which the main acquisition chain is performed by dedicated batch processes, and individual users may run their own client programs in order to observe or modify the acquisition process or control detector parameters.

One of the most basic elements of a real-time acquisition system is hardware that can guarantee a response when data are ready at the inputs. The SLD host computer, a VAX 8800 running VMS, a commercial time-sharing operating system, is not able to guarantee timely response to external inputs. To implement a real-time acquisition using such a host computer, additional hardware is necessary. The choice made was to use a DEC RT MicroVAX running VAXELN, an operating system built for realtime data acquisition and control tasks. The ADC's and control modules reside in three CAMAC crates, which are controlled by a Kinetic Systems 2160 Serial Highway 


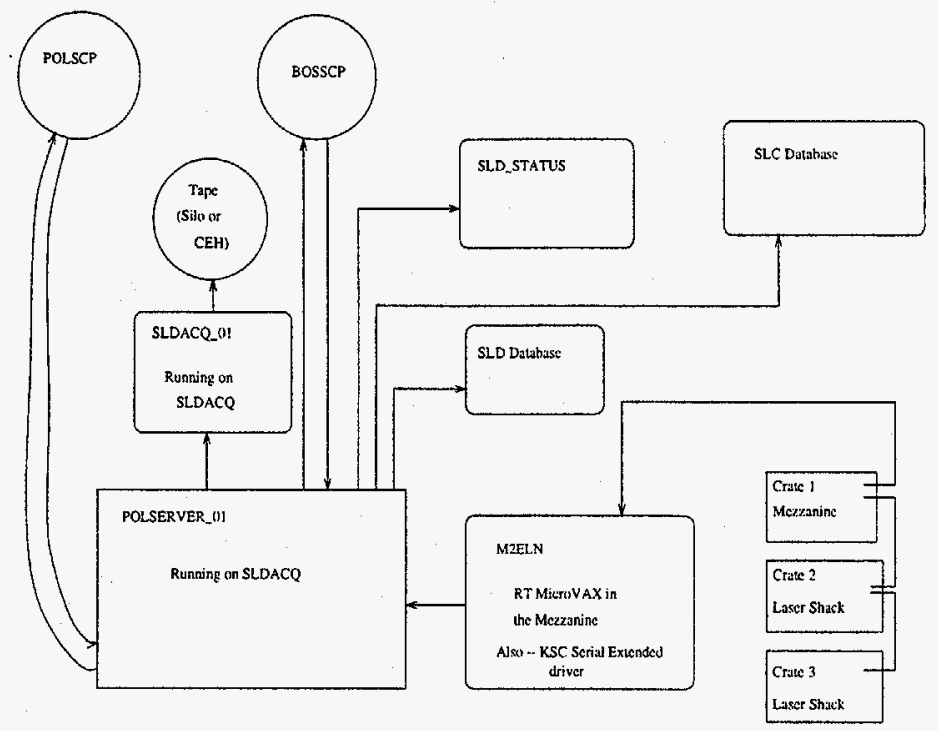

Figure B.1: Schematic diagram of the Compton polarimeter data acquisition.

Driver. One of the crates occupies a space in a rack in the mezzanine of the Collider Experimental Hall ( $\mathrm{CEH}$ ), while the other two are situated in the Compton Laser Shack and are linked by fiber optic cable to the first crate. Each crate has a Kinetic Systems enhanced serial crate controller and a CAMAC list sequencer. On each beam crossing, a pre-loaded list of CAMAC commands executes within each crate, and the resulting data are transferred to the MicroVAX via the Serial Highway Driver.

The MicroVAX accumulates the data in a buffer, and checks to see if the $1 / O$ operätions completed properly and on time. After a fixed number of beam crossings (usually 400), the accumulated buffer is sent to SLDACQ, the host VAX.

A dedicated process on SLDACQ, called POLSERVER, receives the data. It fornss the averages of the signals from each channel of the polarimeter, binned separately for the four $e-\gamma$ helicity combinations and also for the states in which the laser is off. Statistical errors are also formed for each of the averages. After a fixed accumulation time, usually 20,000 beam crossings, the polarization is calculated

\section{B.2 Raw Data}

Also calculated is the quality factor, defined to be the ratio of the mean square of the background-subtracted signal in a polarimeter channel, divided by the mean square deviation of the background from its average value. This was chosen because random fluctuations of the background can make the statistical errors of the measurement poor, especially when the signal strength is poor. If the background is high but very constant on a pulse-to-pulse basis, then the polarization measurement becomes statistically precise. Random fluctuations of the Compton scattering signal contribute the most to the statistical error on the polarization measurement, but these are caused by the temporal and spatial structure of the Compton laser pulse, and are therefore not under the control of shift operators and are omitted from the quality factor. Examples of a typical Cherenkov channel's response during clean running and dirty running are shown in Figures B.2 and B.3.

During the accumulation, raw data is written to tape. It consists of the raw readings for each channel in the acquisition, listed in Table B.1. These are written out only on those pulses on which the laser fires, and one pulse with the laser off for each pulse with the laser on, in order to save data tape, without significantly reducing the statistical power of the average. The averages formed online and the sums written in the summary records contain information from all collected beam crossings.

\section{B.3 Summed Data}

The.averages and errors are calculated for each channel's signal binned on the different helicity states. The beam crossings are subject to data-quality vetoes, though, in order to insure a stable measurement of the polarization. These vetoes consist of:

- Electron Toroid Veto. The reading from the electron toroid at the exiting end of SLD must be at least 45 counts. Pedestal is usually 20 counts and full SLC beam running is $300-800$ counts. 


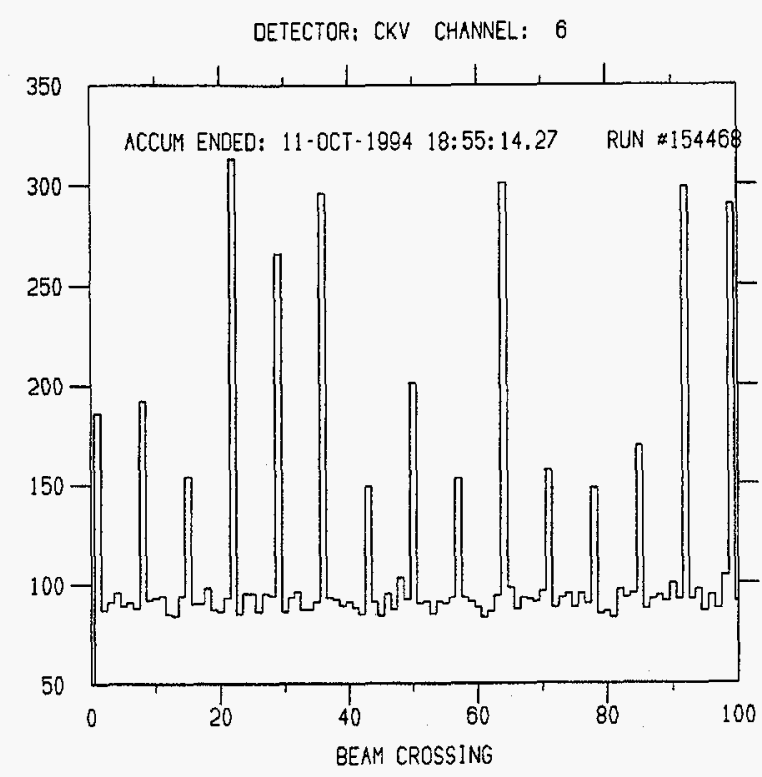

Figure B.2: Raw signal observed in Channel 6 of the Cherenkov detector during clean running.

- Electron Dumper Veto. One of the bits from the register in the mezzanine informs the Compton acquisition if the electron beam has been intentionally dumpered in the BSY. This veto is redundant and weaker than the electron toroid veto.

- Positron Toroid Veto The reading from the positron toroid after the SLD IP should be in excess of 100 counts. The nominal range of positron current readings are the same as for the electrons.

- Excessive Noise Veto This veto compares the signal in Cherenkov channel \# 9 against 1000 counts out of a possible 2048, with a pedestal of $\sim 30$ counts. Channel \# 9 lies off the kinematic endpoint for Compton scattering, and thus measures predominantly accelerator backgrounds with almost no bias for the
OETECTOR: CKV CHANNEL:

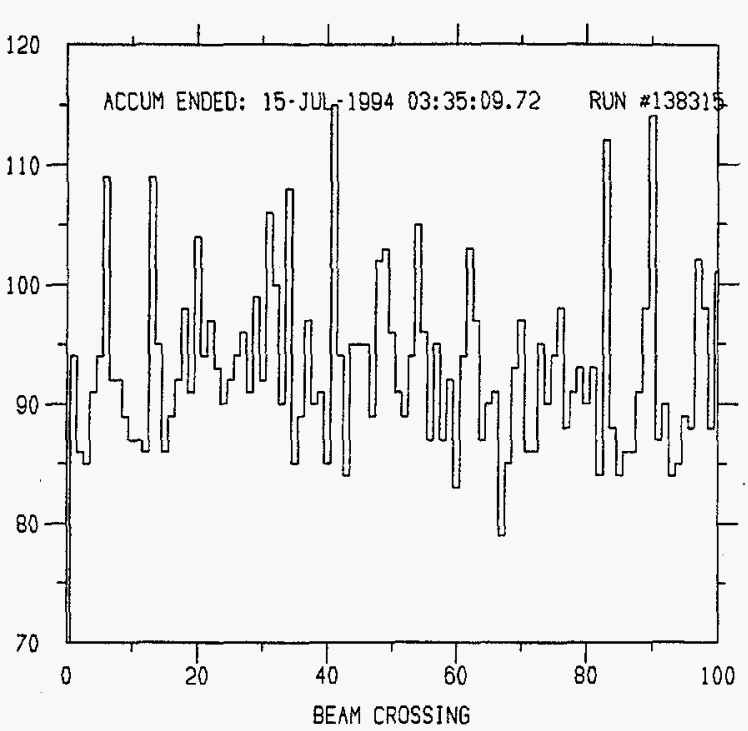

Figure B.3: Raw signal observed in Channel 6 of the Cherenkov detector during dirty running. This particular run had a smaller signal level, contributing to the lack of statistical accuracy on the measurement.

asymmetry measurement. If the other channels saturate, then linearity may be compromised, and the asymmetry affected.

After the requisite number of beam crossings' worth of data has been accumulated. the polarization is calculated and distributed, with its statistical error, to the SLD database, the SLC database, and the SLD status display. In order to be written to the SLC database, the polarization must have a statistical error of $i 5 \%$, as well as having the detector controls set to their nominal positions.

The polarization of the laser light on the optics bench and in the analysis box in the tunnel are also calculated, as are signal ratios in the different channels of the detector. These are all written to the SLD database. The SLD time history process periodically fetches the values from the database and forms a running history. The polarization 
Table B.1: Contents of the raw Compton polarimeter data structure. These can be found in QR94A.TEMPLATE.

\begin{tabular}{|lccc|}
\hline Item & Quantity & Item Size & Size (bytes/beam crossing) \\
\hline Status Word & 1 & 4 & 4 \\
Toroids & 4 & 2 & 16 \\
Polarity Registers & 2 & 2 & 4 \\
Time Digitizers & 4 & 2 & 8 \\
Spare Channels & 5 & 2 & 10 \\
PTD Detector Channels & 16 & 2 & 32 \\
XYZ Polarimeter Channels & 12 & 2 & 24 \\
Cherenkov Channels & 9 & 2 & 18 \\
PMON Information & 2 & 2 & 4 \\
Laser Photodiodes & 15 & 2 & 30 \\
Beamsstrahlung Monitor & 1 & 2 & 2 \\
\hline Total & & & 152 \\
\hline
\end{tabular}

written to the SLD database has to meet the same criteria as those written to the SLC database, although time histories are kept of unfiltered polarization measurements. Often, the electron beam will be shut off in the middle of a Compton measurement. If so, the polarization may be poorly determined and cause a large scatter on a history plot.

A number of quantities are read out of the SLC database regarding the most recent beam-beam deflection scan, collimator settings, source quantum efficiency, and related quantities. These are stored in a QS94A bank, along with the Compton channel averages and RMS's (really sums and summed squares, but information enough to reconstruct the asymmetries and their errors). Time history is not performed for quantities read out of the SLC database, because the SLC time history can be used instead.
The QS94A bank also contains a small amount of raw data at its end. It carries 100 consecutive beam crossings in the same format as QR94A. The consecutive beam crossings are important for offline studies of the random number sequence. The QS94A bank is written to SLD acquisition tape after each Compton measurement, roughly every 3 minutes. The filter job which runs at the end of each SLD run strips the QS94A banks off separately and writes them to files listed in POL.DATACAT.

\section{B.4 Scans}

The first scans implemented for the polarimeter were put in place to enable the targeting of the laser beam on the SLC electron beam to be optimized. These involved scanning the position of the steering lens in the laser transport line, and scanning the timing offset of the laser Q-switch relative to the electron timing signal from SLC. Because of the finite crossing angle of the two beams, these two parameters provide enough degrees of freedom to target the beams, although the timing scan also involved moving the effective Compton IP along the beam axis.

Scans consist of repeatedly taking short runs and changing motors and power supply voltages. The datataking is the same as it is in normal acquisition, except the runs are shorter, the polarization is not calculated, the database writes are disabled, and logging is disabled. The results of the scans are stored in separate buffers for viewing by the SCPs. Targeting scans needed only to retain average signal-background information.

Other scans became necessary as the required precision from the polarimeter increased. The most important of these are the Pockels cell scans. Two varieties were implemented. The first is a quick scan, much like the targeting scans, where short runs-were taken at each of many voltages on the Pockels cells. The statistics retained for these measurements were the laser polarizations on the laser bench and in the analysis box.

The second type involved scanning over the voltages slowly and recording the asymmetry of Compton scattering at each voltage point. Because the necessary time to accumulate enough data to make the measurements significant is large, this scan 
had to be interleaved with nominal data-taking. A typical scan would take roughly one hour.

Other scans were implemented later. Slow scans over phototube voltage and detector table positions provide valuable information about detector linearity and position calibration.

\section{Appendix C}

\section{CDC Vector Hit Finder}

\section{C.1 The need for Vector Hits}

One of the first tasks the SLD reconstruction is faced with is to find the paths of charged tracks in the Central Drift Chamber (CDC). After being fit to helices, these are used directly in analyses, and they are extrapolated both inwards to the Vertex Detector and outward to the CRID and LAC. Ideally, a full fit over all possible combinations of hits in the CDC that maximizes the number and length of tracks found, and minimizes the chisquared of the combined fit, would produce an optimal set of found and fit charged tracks in the chamber. The SLD track fitter is elaborate. though, invoking the general swimmer to take into account multiple scattering and energy loss, and involves the inversion of large matrices at each step of a track fit.

A more efficient solution is to use simpler fitting techniques that can scan over possible combinations of hits quickly and select the best set, which can then be passed to the fitter. This is the approach taken by the SLD pattern recognition. Even this stage, though, would be too cumbersome unless some preprocessing and grouping of locally related hits were not performed.

A natural division of the problem of finding the charged tracks in an event is to divide the hits first into groups by superlayer. Most tracks pass through each superlayer only once, and tracks that bend around in the magnetic field to traverse a superlayer a second time do so at a location distant from the first traversal. Each 
superlayer consists of eight layers of wires, so each track should leave eight hits in each superlayer it traverses. The wires are spaced by $0.5 \mathrm{~cm}$ radially, so the hits from a single track lie nearly on a straight line. The sagitta for a $1 \mathrm{GeV}$ track across a superlayer is $\simeq 36 \mu \mathrm{m}$. By grouping hits, eight at a time (or fewer if they are not all present) into small line segments and allowing the pattern-recognition algorithm use higher-level objects in its search, a large savings in reconstruction effort is effected.

The goals of the vector hit finder, and of the pattern recognition itself, are listed below.

- Maximum efficiency for finding tracks and track segments.

- Maximum purity of the found tracks and segments. Finding background tracks is acceptable and desirable, as long as real tracks are not composed partially of background hits.

- Minimum bias for physics analyses.

The vector hit finder is mainly concerned with the first and second items, as they affect the track-finding efficiency. A perfectly efficient and pure track-finding algorithm will also introduce no more bias for physics than is already present in the chamber.

\section{C.2 Algorithm}

The vector hit $(\mathrm{VH})$ finder breaks the pattern recognition problem down one step further by searching for track segments one cell at a time. A very small fraction of tracks cross cell boundaries, and many of those that do contribute at least one $\mathrm{VH}$ in their layer anyway. The VH finder first finds the drift distance and charge-division $z$ position of each hit in the cell, and arranges them by wire number. Ambiguity partners are listed separately with opposite drift distances*.

"Two common strategies for drift chamber design would help break the drift distance sign ambiguity - staggering the wires and orienting the cells so that the sense wire plane does not contain the electron beam. Neither option was chosen for SLD, although the high spatial resolution partially compensates at the pattern recognition stage.
Because the VH finder is looking for line segments, it seeks first the endpoints, because they provide the most reliable information about the position and direction of the line segment. The search for endpoints starts with the first and last wires in the cell. If a VH cannot be found with endpoints in the first and last layers, combinations of the first and next-to-last layers are tried. Then second and last. Then second and next-to-last, and so forth until the allowed possibilities are exhausted.

A line is drawn between candidate endpoints, and hits on the intervening wires are assigned to the line segment if they are close enough. The criterion is that

$$
\left|d_{\text {hit }}-d_{\text {line }}\right| / \sigma_{\text {hit }}<\sigma_{0},
$$

where $d_{h i t}$ is the signed drift distance from the hit to the wire, $d_{\text {line }}$ is the expected drift distance if the hit were to lie on a line segment containing the two endpoints. $\sigma_{h i t}$ is the drift distance error, and $\sigma_{0}$ is and adjustable parameter of the algorithm, set loosely at 6.0 for the time being. If two hits on a single intervening wire are close enough to the line segment to be added, the closer is chosen.

A list of added hits and their

$$
\chi^{2}=\sum_{\text {addedhits }}\left(d_{\text {hit }}-d_{\text {line }}\right)^{2} / \sigma_{\text {hit }}^{2}
$$

is kept for comparison with other combinations. In addition to trying a straight line segment as a road, a parabolic approximation to a circular segment is also tried to find a set of hits which minimizes the total $\chi^{2}$. The circle used is the one that goes through the two candidate endpoints and the SLD IP. Given the small sagitta for real physics tracks, this additional search provides little discrimination. Very low momentum loopers in the first few layers, mostly the result of synchrotron-induced Compton scatters and beam-wall interactions, however, have noticeable curvature.

The algorithm searches over all combinations of pairs of candidate endpoints and selects the one with the most added intervening hits as a VH. In the case of a tie, the $\mathrm{VH}$ candidate with the smallest $\chi^{2}$ is chosen. If no combination has

$$
n_{\text {hits }} \geq n_{\text {hits,min, }}
$$

where $n_{h i t s, \min }$ is another adjustable parameter, set at 4 hits, then a different pair of wires is chosen to search for candidate endpoints. 


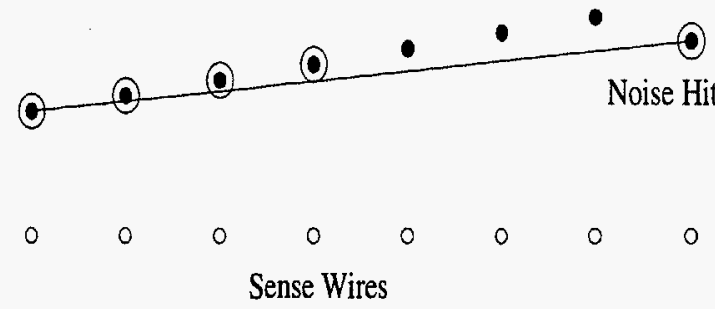

Figure C.1: A vector hit with a noise hit replacing one of the true endpoints.

Because the algorithm uses the endpoints as the seed for VH combinations, it must return and check to see if its seeds are appropriate for the found VH. Once the best combination of hits is found for a given pair of candidate endpoint wires, its hits are fit to a straight line and its $\chi^{2}$ is found. At this point, one of the endpoints is dropped, the remaining hits are fit to a straight line, and the remaining wires that did not contribute hits are scanned for hits that now may lie on the new line segment. If the VH can benefit from dropping one of its endpoints by adding at least one extra hit with a lower chisquared, or by adding more than one extra hit, then the new combination is accepted. The other endpoint is also removed and tested.

The reason for doing this is that if the real endpoint hit were missing from the chamber, and a noise hit were to take its place, then the line segment between a real endpoint hit and the noise one will come in the vicinity of only a fraction of the intervening hits, as diagrammed in Figure C.1. By omitting the endpoint and refitting, more hits may be added and a more accurate vector hit found. If two hits on the end of a VH are noise hits, then the situation is not resolved. In fact, it quickly becomes impossible to distinguish from a real track a string of noise hits that lie on a straight line.

Once a VH is found, its hits are marked as used, and the VH is fit to a line segment and given an absolute position in space given the $\mathrm{CDC}$ wire map. The ambiguity partners of each hit are also marked as used and fit separately for a line segment themselves. Because the drift model incorporates a Lorentz angle correction. the ambiguity partners must be fit separately.
The $z$ coordinate of a VH is an interesting problem, because the charge division resolution is $\sim 6 \mathrm{~cm}$ per hit with a long, non-Gaussian tail. The goal is to arrive at a robust averaging procedure for the charge-division $z$ measurements for each hit on the VH. The previous VH finder's algorithm was to omit the largest $z$ and the smallest $z$ and to average the rest. It was found that often the VH finder would group hits with two on one end of the wire, and six on the other end. It is therefore more reliable to find clusters of $z$ measurements and to average those.

The algorithm used is simple. For each hit in the VH, a count of how many hits have $z$ measurements within $z_{\text {cut }}$ of the hit is made. The parameter $z_{\text {cut }}$ is adjustable and is currently set to $6 \mathrm{~cm}$. The hit with the maximum number of nearby hits is then used to construct a list of close measurements. The hits on that list contribute their $z$ measurements to the average. If no hit has another hit with a $z$ measurement within $z_{\text {cut }}$ of its $z$ measurement, then the VH is claimed to have no $z$ information. Further, if no hits within the VH have reliable charge-division $z$ measurements, usually due to failed electronics on one end of the chamber, then that $\mathrm{VH}$ is also labeled as having no $z$ information. If a VH lacks $z$ information, it is reported as located in the middle of the detector and flagged appropriately.

In addition to finding the average $z$ position of the $\mathrm{VH}$, a dip angle is fit using the charge division information. This dip angle suffers from a large error owing to the measurement error on the $z$ of each hit.

\section{C.3 Performance}

The efficiency of the VH finder, defined in the Monte Carlo to be the probability a VH with a plurality of hits from the Monte Carlo track being investigated is found. The probability is averaged over all Monte Carlo tracks and all layers which the Monte Carlo tracks traverse. This efficiency is found to be $\sim 91 \%$ in hadronic $Z^{0}$ events, largely independent of layer and track momentum, but with worse efficiency as the track $|\cos \theta|$ increases. When it is required that the Monte Carlo track deposit at least 4 hits in the layer in question, the efficiency rises to $\sim 98 \%$, with a smaller drop to $\sim 95 \%$ with increasing $|\cos \theta|$. 

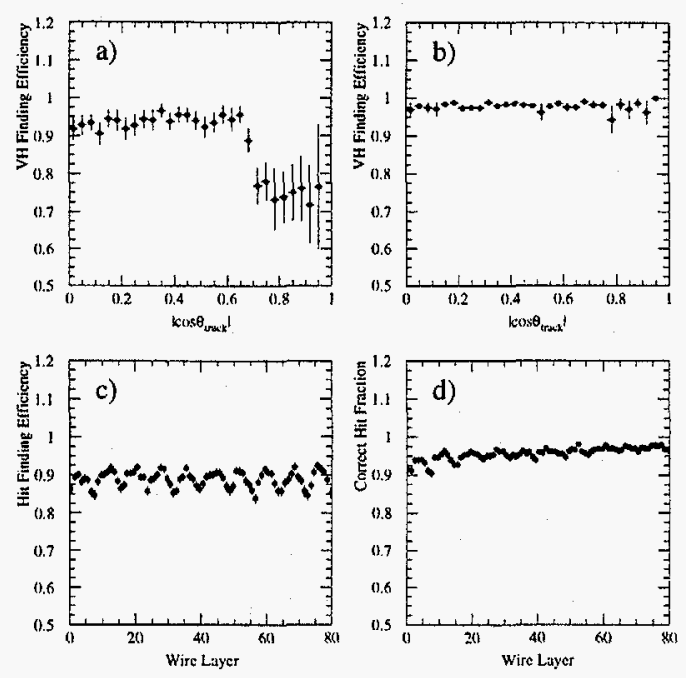

Figure C.2: Performance of the CDC Vector Hit (VH) finder. The probability of finding a VH in a cell where a Monte Carlo track passes is given in Figure a. Figure b shows the same probability if the track is required to have four or more hits in the layer where the $\mathrm{VH}$ is sought. Figure $\mathrm{c}$ shows the hit finding efficiency as a function of wire layer number for found VH's. Structure is seen due to tracks which cross cells and hit loss on the ends of cells. Figure d shows the correct-hit fraction as a function of wire layer.

It is estimated that the losses in efficiency of the VH finder are largely due to correlated hit loss in the CDC. For a track that passes too close to the plane of field wires between cells, each hit can be lost in the dead region. The same is true for a second track which passes through a cell close to another track and also parallel to it. The two-hit resolution as shown in Figure 4.9 indicates that two tracks which pass within $1 \mathrm{~mm}$ of each other will reduce the efficiency of finding the second track's hits. The effect is doubled by the ambiguity of the two halves of a cell. A track will induce two dead regions in a cell - one on its side, and one for its reflection across the sense wire plane. The impact of both of these efficiency losses would be reduced by
Table C.1: Percentages of found Vector Hits, listed by the number of total hits and the number of correct hits, indicating the purities of Vector Hits with different lengths.

\begin{tabular}{|c|cccccccc|}
\hline & \multicolumn{8}{|c|}{ Correct Hits } \\
\hline Total Hits & 1 & 2 & 3 & 4 & 5 & 6 & 7 & 8 \\
\hline 8 & 0.0 & 0.0 & 0.2 & 0.6 & 0.9 & 1.7 & 4.7 & 47.2 \\
7 & 0.0 & 0.0 & 0.2 & 0.7 & 0.7 & 2.1 & 17.6 & \\
6 & 0.0 & 0.0 & 0.4 & 0.6 & 1.0 & 7.3 & & \\
5 & 0.0 & 0.3 & 0.8 & 1.1 & 5.0 & & & \\
4 & 0.2 & 1.0 & 1.2 & 4.5 & & & & \\
\hline
\end{tabular}

"tilting" the cells - arranging the sense and field wire planes so they do not contain the electron beam line. Tilted cells have inefficiencies in the corners, though, but it is less likely to lose all hits in a layer when the geometry is non-projective. Tilted cells would also reduce the impact ambiguity partners have on the pattern recognition stage in dense jets.

One shortcoming of the VH finder as it stands is that it makes no special attempt. to find VH's which cross the field wire plane into another cell. Were this feature to be added, a small fraction $(<5 \%$, and then largely for low-momentum tracks which curve enough to point non-projectively) of the VH's would have their ambiguity remored. The hit-finding efficiency at the ends of the cells is reduced because a fraction of tracks leave the cell near the ends.

The composition of the vector hits is summarized in Table C.1. These are given for a sample of hadronic Monte Carlo events with full simulation and background addition. A hit on a VH which does not come from the track contributing the plurality of hits is counted as a contamination hit. Hits from background, other tracks, and ambiguity partners of correct hits are examples of contamination. The contamination fraction increases as the number of hits present in the VH falls. Missing hits reduce the purity because long VH's are preferred over short ones and the VH finder will look farther to find a hit to add. 


\section{Appendix D}

\section{Trigger and Filter}

\section{D.1 Trigger}

The SLD trigger has several independent components: an energy trigger using the LAC, a tracking trigger which forms candidate track hypotheses in the CDC, a Bhabha trigger using information from the luminosity monitor, and several combinations using information from multiple sources. In addition, there is a "random" trigger, which periodically reads out the entire detector regardless of its contents, and is used for the background overlay events mentioned in Chapter 5 . The trigger types are described in detail in Table D.1.

In addition to the requirements a beam crossing must pass in order for a trigger to fire, there are additional requirements it must pass in order for the readout not to be vetoed. Due to the variable noise environment of SLC, it is important not to read out the detector on a beam crossing in which either beam's bunch has a problem serious enough to cause beam loss close to the detector and fill all of the channels with muons andfor synchrotron radiation. Such an event can be called a "beam splash" event, or a "flyer." Flyers can be a significant source of deadtime when they are frequent, as it takes longer to read out SLD when its occupancy is high. Table D.1 describes these veto conditions.

In 1993 , the CDC Cell overflow veto, $N_{C D C \text { cell }} \geq 275$ was applied to the Hadron as well as the track trigger, and the Energy trigger only read out the calorimetric
Table D.1: Description of the SLD triggers.

\begin{tabular}{|c|c|c|c|c|}
\hline Name & Condition & Prescale & Veto & Readout \\
\hline Random & any crossing & 1 in $2400 \pm 50$ & none & SLD \\
\hline Energy & $\mathrm{KAL}_{h i} \geq 12 \mathrm{GeV}$ & none & $N_{K A L, 10} \geq 1000$ & SLD \\
\hline Bhabha & $\begin{array}{l}\text { Back-to-Back Octants } \\
\text { in LUM } \geq 12 \mathrm{GeV}\end{array}$ & none & none & KAL \\
\hline Track & $\geq 2$ Tracks, Back-to-Back & none & $N_{C D C \text { cell }} \geq 275$ & SLD \\
\hline Hadron & $\geq 1$ Track \& $\mathrm{KAL}_{h i} \geq 4 \mathrm{GeV}$ & none & none & SLD \\
\hline WAB & $\mathrm{KAL}_{h i}(\mathrm{EM}$ only $) \geq 30 \mathrm{GeV}$ & none & $N_{K A L, l o} \geq 1000$ & SLD \\
\hline Muon & $\geq 1$ Track \& WIC CLU & $\leq 5$ in 12000 & $N_{K A L, 10} \geq 1000$ & SLD \\
\hline ELUMMON & $\mathrm{LUM}_{N} \& \mathrm{LUM}_{S} \geq 10 \mathrm{GeV}$ & $1 / 100$ & none & $\mathrm{KAL}$ \\
\hline Cyclic & any crossing & $2 / 120$ & none & SLD \\
\hline
\end{tabular}

Table D.2: LAC layer thresholds and online energy conversions.

\begin{tabular}{|c|c|c|c|}
\hline Layer & High Threshold & Low Threshold & $\mathrm{MeV} /$ Count \\
\hline EM1 and EM2 & 60 & 8 & 4.09 \\
\hline HAD1 and HAD2 & 120 & 12 & 10.81 \\
\hline
\end{tabular}

subsystems of SLD (LAC, WIC Pads, and the LUM). During the "veto period." described in Section 6.2.2, the threshold on the cell count was inappropriately set for the then current definition of how many cells had been hit*.

The terms used in Table D.1 are defined as follows:

$\mathrm{KAL}_{h i}$ is the sum of energy in towers in the LAC above the high threshold. The thresholds and energy scales are described below in Section D.2.1.

$\mathrm{KAL}_{l o}$ is the sum of energy in towers in the LAC above the low threshold The thresholds and energy scales are described below in Section D.2.1.

$N_{K: A L, k i}$ is the number of towers contributing to the $\mathrm{KAL}_{h i}$ energy sum.

$N_{K A L, l o}$ is the number of towers contributing to the $\mathrm{KAL}_{l 0}$ energy sum.

*The cell count was derived from the tracking trigger, which counts nonexistent hardware cells which correspond to the ends of short CDC motherboards as always being hit. A mistally of these put the wrong offset into the CDC hit cell count and the veto threshold was not changed 
$\mathrm{LUM}_{N}$ and $\mathrm{LUM}_{S}$ are the energies deposited in the North and South LUM detectors, respectively.

WIC CLU is the WIC coincidence module, which seeks sets of layers of hits in the WIC. Can be configured to require coincidence of two octants to form a cosmic ray trigger.

The Prescale counts beam crossings after a particular trigger has caused the detector to read out. While the counter is counting its fixed number of beam crossings, that trigger cannot cause another readout. It is built-in deadtime. The Random trigger has a special prescale which randomly jitters the beam crossing to be read out by \pm 50 beam crossings. This is done so as not to lock the random trigger readout to a particular phase of any periodic behavior of SLC.

\section{D.2 Z Filters}

The second stage of event selection is common to all analyses at SLD - the first pass through the raw data with a loose set of event selection criteria. These are designed to select $Z^{0}$ decay events with maximum efficiency, while reducing the number of background triggers passed to the reconstruction and analysis stages. Analyses later apply more restrictive requirements to obtain samples more pure in the decays of interest. There are several filters applied, and if an event passes any one of them, it is included in the filtered data sample available for all SLD experimenters. These consist. of: 1) two calorimetric filters, (EIT and $\mathrm{KZOF}$ ); 2) a first-pass tau filter; and 3) a mu-pair filter. In addition, extra sets are filtered off into non- $Z^{0}$ samples: luminosity. monitor data, $120 \mathrm{~Hz}$ data, random triggers, and Compton polarimeter data.

\section{D.2.1 The EIT Calorimetric Filter}

The EIT filter [144] starts with the subsample of events that pass the energy trigger and imposes stricter requirements:

$$
\begin{aligned}
\text { NEMHI } & \geq 10 \\
E H I & >15 \mathrm{GeV}
\end{aligned}
$$

$$
\begin{aligned}
& E L O<140 \mathrm{GeV} \\
& E H I>1.5 *(E L O-70)
\end{aligned}
$$

where NEMHI is the number of EM towers passing the high threshold, EHI is the total summed energy in the EM and HAD sections for towers passing the high threshold, and ELO is the same sum, but for towers passing the low thresholds. The thresholds and energy scales are defined in Table D.2. The efficiency of this selection has been checked in the sample passed by the KZOF filter and found to be $97.2 \%$ efficient for events also passing KZOF. Its efficiency is estimated at $96 \%$ using the SLD Monte Carlo simulation. The combined trigger and EIT filter efficiency is estimated to be $\sim 93 \%$ [143].

Because the EIT filter is a strict subset of the trigger and a strict subset of the filtered events, and because it is simulated in the Monte Carlo, this analysis requires that events pass the EIT selection in both data and Monte Carlo. If any trigger/filter bias is present, this technique ensures that it is modeled. The inefficiency of the EIT filter for hadronic $Z^{0}$ decay is only $1.8 \%$ within the analysis acceptance, as defined by selection cuts described below, and for tagged events it is also $1.8 \%$. The filter is unlikely to introduce biases to the asymmetry analysis because efficiency effects cancel in the asymmetry ratio.

\section{D.2.2 Additional Filters: Tau, Mu Pair, and KZ0F}

Additional filters are applied during PASS1 reconstruction of events in order to provide data samples for analyses needing two-prong dilepton events and tau erents. These filters by construction pass only events that fail the event selection cuts described in the next section, so they will not be discussed in detail. The KZOF calorimetric sample is also highly correlated with the EIT sample, and allowing events which pass it and not EIT into the analysis increases the size of the event sample by $2 \%$. Its use is not included in this analysis. 


\section{Appendix E}

\section{Monte Carlo Charm Decays}

Listed in this Appendix are the decay tables for the $D^{0}, D^{ \pm}, D_{s}$, and $D^{*}$ mesons and the $\Lambda_{c}^{+}$baryon, as adjusted by Su Dong [129], in order to match better with available data from CLEO, ARGUS, and MARK III. Branching fractions are reported here normalized to unity, to match the implementation in JETSET 7.4.

Table E.1: SLD decay table for the $D^{*+}$ meson.

\begin{tabular}{|lc|}
\hline Mode & Branching Fraction \\
\hline$D^{*+} \rightarrow D^{0} \pi^{+}$ & 0.68100 \\
$D^{*+} \rightarrow D^{+} \pi^{0}$ & 0.30800 \\
$D^{*+} \rightarrow D^{+} \gamma^{+}$ & 0.01100 \\
\hline
\end{tabular}

Table E.2: SLD decay table for the $D^{* 0}$ meson.

\begin{tabular}{|lc|}
\hline Mode & Branching Fraction \\
\hline$D^{* 0} \rightarrow D^{0} \pi^{0}$ & 0.63600 \\
$D^{* 0} \rightarrow D^{0} \gamma$ & 0.36400 \\
\hline
\end{tabular}

Table E.3: SLD decay table for the $D^{0}$ meson.

\begin{tabular}{|c|c|c|c|}
\hline Mode & Branching Fraction & Mode & Branching Fraction \\
\hline$D^{0} \rightarrow K^{-} \pi^{+}$ & 0.04000 & $D^{0} \rightarrow \pi^{0} \pi^{0} \pi^{0} \bar{K}^{0}$ & 0.01500 \\
\hline$D^{0} \rightarrow \pi^{0} \bar{K}^{0}$ & 0.02100 & $D^{0} \rightarrow \bar{K}^{0} \pi^{-} \pi^{+} \pi^{-} \pi^{+}$ & 0.00850 \\
\hline$D^{0} \rightarrow K^{-} K^{+}$ & 0.00410 & $D^{0} \rightarrow \pi^{0} \pi^{0} \bar{K}^{0} \pi^{-} \pi^{+}$ & 0.04000 \\
\hline$D^{0} \rightarrow \bar{K}^{0} K^{0}$ & 0.00110 & $D^{0} \rightarrow K^{-} \pi^{-} \pi^{-} \pi^{+} \pi^{+} \pi^{+}$ & 0.00500 \\
\hline$D^{0} \rightarrow \pi^{-} \pi^{+}$ & 0.00163 & $D^{0} \rightarrow \bar{K}^{0} K^{+} K^{-}$ & 0.00520 \\
\hline$D^{0} \rightarrow \pi^{0} \pi^{0}$ & 0.00013 & $D^{0} \rightarrow \pi^{0} \bar{K}^{0} K^{+} K^{-}$ & 0.00900 \\
\hline$D^{0} \rightarrow \eta \bar{K}^{0}$ & 0.01520 & $D^{0} \rightarrow \phi \pi^{-} \pi^{+}$ & 0.00240 \\
\hline$D^{0} \rightarrow \eta \pi^{0}$ & 0.00008 & $D^{0} \rightarrow K^{+} K^{-} \pi^{+} \pi^{-}$ & 0.00007 \\
\hline$D^{\circ} \rightarrow \eta \eta$ & 0.00016 & $D^{0} \rightarrow K^{0} K^{-} \pi^{+}$ & 0.00220 \\
\hline$D^{0} \rightarrow \eta^{\prime} \bar{K}^{0}$ & 0.01700 & $D^{0} \rightarrow \pi^{0} \pi^{+} \pi^{-}$ & 0.01500 \\
\hline$D^{0} \rightarrow \eta^{\prime} \pi^{0}$ & 0.00004 & $D^{0} \rightarrow \pi^{0} \pi^{-} \pi^{-} \pi^{+} \pi^{+}$ & 0.01700 \\
\hline$D^{0} \rightarrow \rho^{+} K^{-}$ & 0.09000 & $D^{0} \rightarrow \pi^{0} \pi^{0} K^{-} \pi^{-} \pi^{+} \pi^{+}$. & 0.01200 \\
\hline$D^{0} \rightarrow \rho^{0} \bar{K}^{0}$ & 0.00610 & $D^{0} \rightarrow \pi^{0} K^{-} \pi^{-} \pi^{+} \pi^{+}$ & 0.03500 \\
\hline$D^{0} \rightarrow \phi \bar{K}^{0}$ & 0.00880 & $D^{0} \rightarrow \pi^{0} \pi^{0} \pi^{0} K^{-} \pi^{+}$ & 0.01000 \\
\hline$D^{0} \rightarrow K^{*-} \pi^{+}$ & 0.04500 & $D^{0} \rightarrow \rho^{0} \mathrm{~K}^{-} \pi^{+}$ & 0.00630 \\
\hline$D^{0} \rightarrow K^{*} \pi^{0}$ & 0.02100 & $D^{0} \rightarrow \bar{K}^{* 0} \pi^{+} \pi^{-}$ & 0.01600 \\
\hline$D^{0} \rightarrow \phi \pi^{0}$ & 0.00026 & $D^{0}-\bar{K}^{* 0} \pi^{0} \pi^{+} \pi^{-}$ & 0.01600 \\
\hline$D^{0} \rightarrow \omega \bar{K}^{0}$ & 0.02500 & $D^{0} \rightarrow \pi^{0} \mathrm{~K}^{-} \mathrm{K}^{+} \pi^{+} \pi^{-}$ & 0.00280 \\
\hline$D^{0} \rightarrow K^{*-} K^{+}$ & 0.00200 & $D^{0} \rightarrow e^{+} \nu_{e} K^{-}$ & 0.03500 \\
\hline$D^{0} \rightarrow K^{*+} K^{-}$ & 0.00350 & $D^{0} \rightarrow e^{+} \nu_{e} K^{*-}$ & 0.02500 \\
\hline$D^{0} \rightarrow K^{*-} \rho^{+}$ & 0.06200 & $D^{0} \rightarrow e^{+} \nu_{e} \bar{K}^{0} \pi^{-}$ & 0.00200 \\
\hline$D^{0} \rightarrow K^{-} a_{1}^{+}$ & 0.07400 & $D^{0} \rightarrow e^{+} \nu_{e} K^{-} \pi^{0}$ & 0.00200 \\
\hline$D^{0} \rightarrow K_{1}^{-} \pi^{+}$ & 0.01090 & $D^{0} \rightarrow e^{+} \nu_{e} \bar{K}^{* 0} \pi^{-}$ & 0.00400 \\
\hline$D^{0} \rightarrow \bar{K}^{0} a_{1}^{0}$ & 0.00420 & $D^{0} \rightarrow e^{+} \nu_{e} K^{*-} \pi^{0}$ & 0.00400 \\
\hline$D^{0}-\bar{K}_{1}^{* 0} \pi^{0}$ & 0.00820 & $D^{0}-e^{+} \nu_{e} \pi^{-}$ & $\dot{0} .00300$ \\
\hline$D^{0} \rightarrow \rho^{0} \overline{K^{*}}$ & 0.01500 & $D^{0} \rightarrow e^{+} \nu_{e} \rho^{-}$ & 0.00200 \\
\hline$D^{0} \rightarrow \bar{K}^{* 0} \eta$ & 0.02100 & $D^{0} \rightarrow \mu^{+} \nu_{\mu} K^{-}$ & 0.03500 \\
\hline$D^{0} \rightarrow \bar{K}^{* 0} K^{* 0}$ & 0.00270 & $D^{0} \rightarrow \mu^{+} \nu_{\mu} K^{*-}$ & 0.02500 \\
\hline$D^{0} \rightarrow \pi^{0} K-\pi^{+}$ & 0.01100 & $D^{0} \rightarrow \mu^{+} \nu_{\mu} \bar{K}^{0} \pi^{-}$ & 0.00200 \\
\hline$\tilde{D}^{0} \rightarrow \bar{K}^{0} \pi^{-} \pi^{+}$ & 0.01800 & $D^{0}-\mu^{+} \nu_{\mu} K^{-} \pi^{0}$ & 0.00200 \\
\hline$D^{0} \rightarrow \pi^{0} \pi^{0} \bar{K}^{0}$ & 0.01200 & $D^{0} \rightarrow \mu^{+} \nu_{\mu} \overline{K^{* 0}} \pi^{-}$ & 0.00400 \\
\hline$D^{0} \rightarrow K^{-} \pi^{-} \pi^{+} \pi^{+}$ & 0.01800 & $D^{0} \rightarrow \mu^{+} \nu_{\mu} K^{*-} \pi^{0}$ & 0.00400 \\
\hline$D^{0} \rightarrow \pi^{0} \pi^{0} K^{-} \pi^{+}$ & 0.04000 & $D^{0} \rightarrow \mu^{+} \nu_{\mu} \pi^{-}$ & 0.00300 \\
\hline$D^{\circ} \rightarrow \pi^{+} \pi^{-} \pi^{-} \pi^{+}$ & 0.00750 & $D^{0} \rightarrow \mu^{+} \nu_{\mu} \rho^{-}$ & 0.00200 \\
\hline$D^{0} \rightarrow \pi^{0} \bar{K}^{0} \pi^{-} \pi^{+}$ & 0.02200 & & \\
\hline
\end{tabular}


Table E.4: SLD decay table for the $D^{+}$meson.

\begin{tabular}{|c|c|c|c|}
\hline Mode & Branching Fraction & Mode & Branching Fraction \\
\hline$D^{+} \rightarrow \bar{K}^{0} \pi^{+}$ & 0.02455 & $D^{+} \rightarrow \rho^{+} K^{-} \pi^{+}$ & 0.00755 \\
\hline$D^{+} \rightarrow \bar{K}^{0} K^{+}$ & 0.00689 & $D^{+} \rightarrow \rho^{+} \pi^{0} K^{0}$ & 0.0075 .5 \\
\hline$D^{+} \rightarrow \pi^{0} \pi^{+}$ & 0.00283 & $D^{+} \rightarrow \bar{K}^{* 0} \pi^{-} \pi^{+} \pi^{+}$ & 0.00718 \\
\hline$D^{+} \rightarrow \eta \pi^{+}$ & 0.00623 & $D^{+} \rightarrow \pi^{0} K^{-} K^{+} \pi^{+}$ & 0.01416 \\
\hline$D^{+} \rightarrow \eta^{\prime} \pi^{+}$ & 0.00378 & $D^{+} \rightarrow \rho^{0} \widetilde{K}^{* 0} \pi^{+}$ & 0.00538 \\
\hline$D^{+} \rightarrow \overline{\boldsymbol{K}}^{* 0} \pi^{+}$ & 0.01794 & $D^{+} \rightarrow \pi^{0} \pi^{+} \pi^{-} \pi^{+}$ & 0.01605 \\
\hline$D^{+} \rightarrow \rho^{+} \bar{K}^{0}$ & 0.06231 & $D^{+} \rightarrow \pi^{+} \pi^{-} \pi^{+} \pi^{-} \pi^{+}$ & 0.00142 \\
\hline$D^{+} \rightarrow \phi \pi^{+}$ & 0.00566 & $D^{+} \rightarrow \pi^{0} \pi^{-} \pi^{-} \pi^{+} \pi^{+} \pi^{+}$ & 0.00264 \\
\hline$D^{+} \rightarrow \bar{K}^{* 0} K^{+}$ & 0.00444 & $D^{+} \rightarrow e^{+} \nu_{e} \bar{K}^{0}$ & 0.07553 \\
\hline$D^{+} \rightarrow \bar{K}^{* 0} K^{*+}$ & 0.02455 & $D^{+} \rightarrow e^{+} \nu_{e} \bar{K}^{* 0}$ & 0.05287 \\
\hline$D^{+} \rightarrow \rho^{+} \pi^{0}$ & 0.00566 & $D^{+} \rightarrow e^{+} \nu_{e} \bar{K}^{0} \pi^{0}$ & 0.00566 \\
\hline$D^{+} \rightarrow \rho^{+} \eta$ & 0.00566 & $D^{+} \rightarrow e^{+} \nu_{e} K^{-} \pi^{+}$ & 0.00566 \\
\hline$D^{+} \rightarrow \rho^{+} \eta^{\prime}$ & 0.00378 & $D^{+} \rightarrow e^{+} \nu_{\varepsilon} \bar{K}^{* 0} \pi^{0}$ & 0.01039 \\
\hline$D^{+} \rightarrow \bar{K}^{* 0} \rho^{+}$ & 0.01983 & $D^{+} \rightarrow e^{+} \nu_{e} K^{*-} \pi^{+}$ & 0.01039 \\
\hline$D^{+} \rightarrow \bar{K}^{0} a_{1}^{+}$ & 0.07081 & $D^{+} \rightarrow e^{+} \nu_{\varepsilon} \pi^{0}$ & 0.00566 \\
\hline$D^{+} \rightarrow \bar{K}_{1}^{* 0} \pi^{+}$ & 0.04154 & $D^{+} \rightarrow e^{+} \nu_{e} \eta$ & 0.00094 \\
\hline$D^{+} \rightarrow K^{-} \pi^{+} \pi^{+}$ & 0.07175 & $D^{+} \rightarrow e^{+} \nu_{e} \eta^{\prime}$ & 0.00094 \\
\hline$D^{+} \rightarrow \pi^{0} \bar{K}^{0} \pi^{+}$ & 0.01133 & $D^{+} \rightarrow e^{+} \nu_{e} \rho^{0}$ & 0.00189 \\
\hline$D^{+} \rightarrow K^{+} K^{-} \pi^{+}$ & 0.00378 & $D^{+} \rightarrow \epsilon^{+} \nu_{e} \omega$ & 0.00094 \\
\hline$D^{+} \rightarrow \bar{K}^{0} \bar{K}^{0} K^{+}$ & 0.02549 & $D^{+} \rightarrow \mu^{+} \nu_{\mu} \bar{K}^{0}$ & 0.07553 \\
\hline$D^{+} \rightarrow \phi \pi^{0} \pi^{+}$ & 0.02266 & $D^{+} \rightarrow \mu^{+} \nu_{\mu} \bar{K}^{*} * 0$ & 0.05287 \\
\hline$D^{+} \rightarrow \pi^{+} \pi^{-} \pi^{+}$ & 0.00264 & $D^{+} \rightarrow \mu^{+} \nu_{\mu} \bar{K}^{0} \pi^{0}$ & 0.00566 \\
\hline$D^{+} \rightarrow \pi^{0} \pi^{0} \pi^{+}$ & 0.01048 & $D^{+} \rightarrow \mu^{+} \nu_{\mu} K^{-} \pi^{+}$ & 0.00566 \\
\hline$D^{+} \rightarrow \bar{K}^{0} \pi^{-} \pi^{+} \pi^{+}$ & 0.00755 & $D^{+} \rightarrow \mu^{+} \nu_{\mu} \bar{K}^{* 0} \pi^{0}$ & 0.01039 \\
\hline$D^{+} \rightarrow \pi^{0} K^{-} \pi^{+} \pi^{+}$ & 0.00850 & $D^{+} \rightarrow \mu^{+} \nu_{\mu} K^{*-} \pi^{+}$ & 0.01039 \\
\hline$D^{+} \rightarrow \pi^{0} \pi^{0} \bar{K}^{0} \pi^{+}$ & 0.01888 & $D^{+} \rightarrow \mu^{+} \nu_{\mu} \pi^{0}$ & 0.00566 \\
\hline$D^{+} \rightarrow K^{-} \pi^{-} \pi^{+} \pi^{+} \pi^{+}$ & 0.00576 & $D^{+} \rightarrow \mu^{+} \nu_{\mu} \eta$ & 0.00094 \\
\hline$D^{+} \rightarrow{\overline{K^{0}}}^{0} \pi^{-} \pi^{+} \pi^{-} \pi^{+} \pi^{+}$ & 0.00094 & $D^{+} \rightarrow \mu^{+} \nu_{\mu} \eta^{\prime}$ & 0.00094 \\
\hline$D^{+} \rightarrow \pi^{0} \pi^{0} K^{-} \pi^{+} \pi^{+}$ & 0.02077 & $D^{+} \rightarrow \mu^{+} \nu_{\mu} \rho^{0}$ & 0.00189 \\
\hline$D^{+\cdot} \rightarrow \pi^{0} \bar{K}^{0} \pi^{-} \pi^{+} \pi^{+}$ & 0.05098 & $D^{+} \rightarrow \mu^{+} \nu_{\mu} \omega$ & 0.00094 \\
\hline$D^{+} \rightarrow \pi^{0} \pi^{0} \pi^{0} \bar{K}^{0} \pi^{+}$ & 0.02832 & & \\
\hline
\end{tabular}

Table E.5: SLD decay table for the $D_{s}^{+}$meson, Part 1 .

\begin{tabular}{|c|c|c|c|}
\hline Mode & Branching Fraction & Mode & Branching Fraction \\
\hline$\overline{D_{s}^{+}} \rightarrow \eta \pi^{+}$ & 0.01349 & $D_{s}^{+} \rightarrow K^{*-} K^{+} \pi^{+}$ & 0.00682 \\
\hline$D_{s}^{+} \rightarrow \eta^{\prime} \pi^{+}$ & 0.03328 & $D_{s}^{+} \rightarrow K^{*+} K^{-} \pi^{+}$ & 0.00682 \\
\hline$D_{s}^{+} \rightarrow K^{0} \pi^{+}$ & 0.00259 & $D_{s}^{+} \rightarrow \rho^{+} K^{0} K^{0}$ & 0.00189 \\
\hline$D_{s}^{+} \rightarrow \bar{K}^{0} K^{+}$ & 0.02519 & $D_{s}^{+} \rightarrow \rho^{+} K^{-} K^{+}$ & 0.00203 \\
\hline$D_{s}^{+} \rightarrow \pi^{0} K^{+}$ & 0.00040 & $D_{s}^{+} \rightarrow K^{* 0} \bar{K}^{0} \pi^{+}$ & 0.00670 \\
\hline$D_{s}^{+} \rightarrow \eta K^{+}$ & 0.00020 & $D_{s}^{+} \rightarrow \bar{K}^{* 0} K^{0} \pi^{+}$ & 0.00670 \\
\hline$D_{s}^{+} \rightarrow \rho^{+} \eta$ & 0.07106 & $D_{s}^{+} \rightarrow \rho^{0} \bar{K}^{0} K^{+}$ & 0.00196 \\
\hline$D_{s}^{+} \rightarrow \rho^{+} \eta^{\prime}$ & 0.08545 & $D_{s}^{+} \rightarrow \omega \bar{K}^{0} K^{+}$ & 0.00174 \\
\hline$D_{s}^{+} \rightarrow \phi \pi^{+}$ & 0.03148 & $D_{s}^{+} \rightarrow \bar{K}^{* 0} \pi^{0} K^{+}$ & 0.00692 \\
\hline$D_{s}^{+} \rightarrow \rho^{+} K^{0}$ & 0.00499 & $D_{s}^{+} \rightarrow K^{*+} \pi^{0} \bar{K}^{0}$ & 0.00679 \\
\hline$D_{s}^{+} \rightarrow K^{* 0} \pi^{+}$ & 0.00143 & $D_{s}^{+} \rightarrow \rho^{0} K^{0} \pi^{+}$ & 0.00058 \\
\hline$D_{s}^{+} \rightarrow{\overline{K^{*}}}^{* 0} K^{+}$ & 0.02968 & $D_{s}^{+} \rightarrow \rho^{0} \pi^{0} K^{+}$ & 0.00059 \\
\hline$D_{s}^{+} \rightarrow K^{*}+\bar{K}^{0}$ & 0.02968 & $D_{s}^{+} \rightarrow \rho^{+} \pi^{0} K^{0}$ & 0.00058 \\
\hline$D_{s}^{+} \rightarrow \omega K^{+}$ & 0.00068 & $D_{s}^{+} \rightarrow \rho^{+} K^{+} \pi^{-}$ & 0.00058 \\
\hline$D_{s}^{+} \rightarrow K^{*+} \pi^{0}$ & 0.00023 & $D_{s}^{+} \rightarrow \rho^{-} K^{+} \pi^{+}$ & 0.00058 \\
\hline$D_{s}^{+} \rightarrow K^{*+} \eta$ & 0.00067 & $D_{s}^{+} \rightarrow \omega \pi^{0} K^{+}$ & 0.00057 \\
\hline$D_{s}^{+} \rightarrow K^{*+} \eta^{\prime}$ & 0.00079 & $D_{s}^{+}-\omega K^{0} \pi^{+}$ & 0.00056 \\
\hline$D_{s}^{+} \rightarrow \dot{\phi} K^{+}$ & 0.00001 & $D_{s}^{+}-\phi K^{0} \pi^{+}$ & 0.00017 \\
\hline$D_{s}^{+}-f_{0}^{0} \pi^{+}$ & 0.00702 & $D_{s}^{+} \rightarrow \phi \pi^{0} K^{+}$ & 0.00018 \\
\hline$D_{s}^{+} \rightarrow \eta a_{1}^{+}$ & 0.01284 & $D_{s}^{+} \rightarrow K^{*+} \pi^{-} \pi^{+}$ & 0.00098 \\
\hline$D_{s}^{+} \rightarrow K^{0} a_{1}^{+}$ & 0.00183 & $D_{s}^{+} \rightarrow K^{*+} \pi^{0} \pi^{0}$ & 0.00099 \\
\hline$D_{s}^{+} \rightarrow K^{+} a_{1}^{0}$ & 0.00026 & $D_{s}^{+} \rightarrow K^{*+} \bar{K}^{0} K^{0}$ & 0.00002 \\
\hline$D_{s}^{+} \rightarrow \phi \rho^{+}$ & 0.04677 & $D_{s}^{+} \rightarrow K^{*+} K^{-} K^{+}$ & 0.00002 \\
\hline$D_{s}^{+} \rightarrow K^{* 0} \rho^{+}$ & 0.00194 & $D_{s}^{+} \rightarrow K^{* 0} \pi^{0} \pi^{+}$ & 0.00099 \\
\hline$D_{s}^{+} \rightarrow \bar{K}^{* 0} K^{*+}$ & 0.04498 & $D_{s}^{+} \rightarrow K^{* 0} \bar{K}^{-0} K^{+}$ & 0.00002 \\
\hline
\end{tabular}


Table E.6: SLD decay table for the $D^{+}$meson, Part 2.

\begin{tabular}{|c|c|c|c|}
\hline Mode & Branching Fraction & Mode & Branching Fraction \\
\hline$D_{s}^{+} \rightarrow \rho^{0} K^{*+}$ & 0.00029 & $D_{s}^{+} \rightarrow \bar{K}^{* 0} K^{0} K^{+}$ & 0.00002 \\
\hline$D_{s}^{+} \rightarrow \omega K^{*+}$ & 0.00028 & $D_{s}^{+} \rightarrow K^{*-} K^{+} K^{+}$ & 0.00002 \\
\hline$D_{s}^{+} \rightarrow \phi K^{*+}$ & 0.00022 & $D_{s}^{+} \rightarrow \rho^{+} \pi^{0} \pi^{0}$ & 0.01069 \\
\hline$D_{s}^{+} \rightarrow e^{+} \nu_{e} \eta$ & 0.02788 & $D_{s}^{+} \rightarrow \omega \pi^{0} \pi^{+}$ & 0.01032 \\
\hline$D_{s}^{+} \rightarrow e^{+} \nu_{e} \eta^{\prime}$ & 0.03328 & $D_{s}^{+} \rightarrow \rho^{0} \pi^{0} \pi^{+}$ & 0.01062 \\
\hline$D_{s}^{+} \rightarrow e^{+} \nu_{e} \phi$ & 0.01799 & $D_{s}^{+} \rightarrow \rho^{+} \pi^{-} \pi^{+}$ & 0.01056 \\
\hline$D_{s}^{+} \rightarrow e^{+} \nu_{e} K^{-0}$ & 0.00180 & $D_{s}^{+}-\rho^{-} \pi^{+} \pi^{+}$ & 0.01056 \\
\hline$D_{s}^{+} \rightarrow e^{+} \nu_{e} K^{* 0}$ & 0.00180 & $D_{s}^{+} \rightarrow \phi \pi^{+} \pi^{-} \pi^{+}$ & 0.01079 \\
\hline$D_{s}^{+} \rightarrow \mu^{+} \nu_{\mu} \eta$ & 0.02788 & $D_{s}^{+} \rightarrow \eta \pi^{+} \pi^{-} \pi^{+}$ & 0.02008 \\
\hline$D_{s}^{+} \rightarrow \mu^{+} \nu_{\mu} \eta^{\prime}$ & 0.03328 & $D_{3}^{+} \rightarrow \eta^{\prime} \pi^{+} \pi^{-} \pi^{+}$ & 0.02008 \\
\hline$D_{s}^{+} \rightarrow \mu^{+} \nu_{\mu} \phi$ & 0.01799 & $D_{s}^{+} \rightarrow \phi \pi^{0} \pi^{0} \pi^{+}$ & 0.02008 \\
\hline$D_{s}^{+} \rightarrow \mu^{+} \nu_{\mu} K^{-0}$ & 0.00180 & $D_{s}^{+} \rightarrow \eta \pi^{0} \pi^{0} \pi^{+}$ & 0.02008 \\
\hline$D_{s}^{+} \rightarrow \mu^{+} \nu_{\mu} K^{* 0}$ & 0.00180 & $D_{s}^{+} \rightarrow \eta^{\prime} \pi^{0} \pi^{0} \pi^{+}$ & 0.02008 \\
\hline$D_{s}^{+} \rightarrow \nu_{\tau} \tau^{+}$ & 0.01799 & $D_{s}^{+} \rightarrow \bar{K}^{0} K^{+} \pi^{+} \pi^{-}$ & 0.01101 \\
\hline$D_{s}^{+} \rightarrow K^{+} K^{-} \pi^{+}$ & 0.00729 & $D_{s}^{+} \rightarrow \pi^{0} \pi^{0} \bar{K}^{0} K^{+}$ & 0.01101 \\
\hline$D_{s}^{+} \rightarrow K^{0} \bar{K}^{0} \pi^{+}$ & 0.00816 & $D_{s}^{+} \rightarrow \phi \pi^{0} \pi^{+} \pi^{-} \pi^{+}$ & 0.00680 \\
\hline$D_{s}^{+} \rightarrow \pi^{0} K^{0} K^{+}$ & 0.00828 & $D_{s}^{+} \rightarrow \eta \pi^{0} \pi^{+} \pi^{-} \pi^{+}$ & 0.01004 \\
\hline$D_{s}^{+} \rightarrow \eta \bar{K}^{0} K^{+}$ & 0.00268 & $D_{s}^{+} \rightarrow \eta^{\prime} \pi^{0} \pi^{+} \pi^{-} \pi^{+}$ & 0.01004 \\
\hline$D_{s}^{+} \rightarrow \pi^{0} K^{0} \pi^{+}$ & 0.00076 & $D_{s}^{+} \rightarrow \phi \pi^{0} \pi^{0} \pi^{0} \pi^{+}$ & 0.00680 \\
\hline$D_{s}^{+} \rightarrow K^{+} \pi^{-} \pi^{+}$ & 0.00126 & $D_{s}^{+} \rightarrow \eta \pi^{0} \pi^{0} \pi^{0} \pi^{+}$ & 0.01004 \\
\hline$D_{s}^{+} \rightarrow \pi^{0} \pi^{0} K^{+}$ & 0.00076 & $D_{s}^{+}-\eta^{\prime} \pi^{0} \pi^{0} \pi^{0} \pi^{+}$ & 0.01004 \\
\hline$D_{s}^{+} \rightarrow K^{0} \bar{K}^{0} K^{+}$ & 0.00017 & $D_{s}^{+} \rightarrow \pi^{0} \bar{K}^{0} K^{+} \pi^{+} \pi^{-}$ & 0.00550 \\
\hline$D_{s}^{+} \rightarrow K^{+} K^{+} K^{-}$ & 0.00018 & $D_{s}^{+} \rightarrow \pi^{0} \pi^{0} \pi^{0} \bar{K}^{0} K^{+}$ & 0.00550 \\
\hline$D_{3}^{+} \rightarrow \pi^{0} \pi^{0} \pi^{+}$ & 0.00919 & $D_{3}^{+} \rightarrow K^{+} K^{-} \pi^{-} \pi^{+} \pi^{+}$ & 0.00171 \\
\hline$D_{s}^{+} \rightarrow \pi^{+} \pi^{-} \pi^{+}$ & 0.00720 & $D_{s}^{+} \rightarrow \pi^{+} \pi^{-} \pi^{+} \pi^{-} \pi^{+}$ & 0.00171 \\
\hline$D_{s}^{+} \rightarrow \phi \pi^{0} \pi^{+}$ & 0.01290 & & \\
\hline
\end{tabular}

Table E.7: SLD decay table for the $\Lambda_{c}^{+}$baryon, Part 1 .

\begin{tabular}{|c|c|c|c|}
\hline Mode & Branching Fraction & Mode & Branching Fraction \\
\hline$\Lambda_{c}^{+} \rightarrow \mathrm{p} \bar{K}^{0}$ & 0.02175 & $\Lambda_{c}^{+} \rightarrow \mathrm{p} \bar{K}^{0} K^{+} \pi^{-}$ & 0.00104 \\
\hline$\Lambda_{c}^{+}-\Lambda^{0} \pi^{+}$ & 0.00818 & $\Lambda_{c}^{+} \rightarrow \mathrm{p} K^{0} K^{-} \pi^{+}$ & 0.00104 \\
\hline$\Lambda_{c}^{+} \rightarrow \Sigma^{0} \pi^{+}$ & 0.00901 & $\Lambda_{c}^{+} \rightarrow \mathrm{p} \pi^{0} \bar{K}^{0} K^{0}$ & 0.00104 \\
\hline$\Lambda_{c}^{+} \rightarrow \Sigma^{+} \pi^{0}$ & 0.00311 & $\Lambda_{c}^{+} \rightarrow \Lambda^{0} K^{+} \pi^{+} \pi^{-}$ & 0.00414 \\
\hline$\Lambda_{c}^{+} \rightarrow \mathrm{p} \pi^{0}$ & 0.00155 & $\Lambda_{c}^{+} \rightarrow \Lambda^{0} \pi^{0} \pi^{0} K^{+}$ & 0.00207 \\
\hline$\Lambda_{c}^{+} \rightarrow \mathrm{n} \pi^{+}$ & 0.00311 & $\Lambda_{c}^{+} \rightarrow \Lambda^{0} \pi^{0} K^{0} \pi^{+}$ & 0.00414 \\
\hline$\Lambda_{c}^{+} \rightarrow \Sigma^{+} \eta$ & 0.00311 & $\Lambda^{+} \rightarrow \Sigma^{0} K^{+} \pi^{+} \pi^{-}$ & 0.00104 \\
\hline$\Lambda_{c}^{+}-f_{0}^{0} \mathrm{p}$ & 0.00186 & $\Lambda_{c}^{+} \rightarrow \Sigma^{0} \pi^{0} K^{0} \pi^{+}$ & 0.00104 \\
\hline$\Lambda_{c}^{+} \rightarrow \mathrm{p} \phi$ & 0.00135 & $\Lambda^{+} \rightarrow \Sigma^{+} \pi^{0} K^{+} \pi^{-}$ & 0.00104 \\
\hline$\Lambda_{c}^{+}-\Delta^{++} K^{-}$ & 0.00684 & $\Lambda_{c}^{+}-\Sigma-\pi^{0} K^{+} \pi^{+}$ & 0.00104 \\
\hline$\Lambda_{c}^{+} \rightarrow \mathrm{p} K^{-} \pi^{+}$ & 0.04557 & $\Lambda_{c}^{+}-\mathrm{p} \pi^{0} \pi^{0} K^{-} \pi^{+}$ & 0.01554 \\
\hline$\Lambda_{c}^{+} \rightarrow \mathrm{p} \pi^{0} \bar{K}^{0}$ & 0.03314 & $\Lambda_{c}^{+} \rightarrow \mathrm{p} K^{-} \pi^{+} \pi^{+} \pi^{-}$ & 0.00073 \\
\hline$\Lambda_{c}^{+} \rightarrow \mathrm{n} \bar{K}^{0} \pi^{+}$ & 0.04557 & $\Lambda_{c}^{+} \rightarrow \mathrm{p} \pi^{-} \pi^{+} \pi^{-} \pi^{+}$ & 0.00124 \\
\hline$\Lambda_{c}^{+} \rightarrow \Lambda^{0} \pi^{0} \pi^{+}$ & 0.01657 & $\Lambda_{c}^{+} \rightarrow \mathrm{p} \pi^{0} \pi^{0} \pi^{0} \bar{K}^{0}$ & 0.01554 \\
\hline$\Lambda_{c}^{+} \rightarrow \Sigma^{0} \pi^{0} \pi^{+}$ & 0.01554 & $\Lambda_{c}^{+} \rightarrow \mathrm{p} \pi^{0} \bar{K}^{0} \pi^{+} \pi^{-}$ & 0.03107 \\
\hline$\Lambda_{c}^{+} \rightarrow \Sigma^{+} \pi^{0} \pi^{0}$ & 0.01554 & $\Lambda_{c}^{+} \rightarrow \mathrm{n} \pi^{0} \pi^{0}{\overline{K^{0}}}^{0} \pi^{+}$ & 0.015 .54 \\
\hline$\Lambda_{c}^{+}-\Sigma^{+} \pi^{-} \pi^{+}$ & 0.03107 & $\Lambda_{c}^{+} \rightarrow \mathrm{n} \bar{K}^{0} \pi^{+} \pi^{+} \pi^{-}$ & 0.01554 \\
\hline$\Lambda_{c}^{+} \rightarrow \Sigma^{-} \pi^{+} \pi^{+}$ & 0.01657 & $\Lambda_{c}^{+}-\mathrm{n} \pi^{0} K^{-} \pi^{+} \pi^{+}$ & 0.01554 \\
\hline$\Lambda_{c}^{+} \rightarrow \mathrm{p} \pi^{-} \pi^{+}$ & 0.00228 & $\Lambda_{c}^{+} \rightarrow \Lambda^{0} \pi^{0} \pi^{0} \pi^{0} \pi^{+}$ & 0.02382 \\
\hline$\Lambda_{c}^{+} \rightarrow \mathrm{p} \pi^{0} \pi^{0}$ & 0.00259 & $\Lambda_{c}^{+} \rightarrow \Lambda^{0} \pi^{0} \pi^{-} \pi^{+} \pi^{+}$ & 0.04661 \\
\hline$\Lambda_{c}^{+} \rightarrow \mathrm{p} K^{-} K^{+}$ & 0.00166 & $\Lambda_{c}^{+} \rightarrow \Sigma^{0} \pi^{0} \pi^{0} \pi^{0} \pi^{+}$ & 0.00621 \\
\hline$\Lambda_{c}^{+} \rightarrow \mathrm{p} \bar{K}^{0} K^{0}$ & 0.00311 & $\Lambda_{c}^{+} \rightarrow \Sigma^{0} \pi^{0} \pi^{-} \pi^{+} \pi^{+}$ & 0.01243 \\
\hline$\Lambda_{c}^{+} \rightarrow \mathrm{n} \pi^{0} \pi^{+}$ & 0.00311 & $\Lambda_{c}^{+} \rightarrow \Sigma^{+} \pi^{0} \pi^{0} \pi^{0} \pi^{0}$ & 0.00621 \\
\hline$\Lambda_{c}^{+} \rightarrow n \bar{K}^{0} K^{+}$ & 0.00259 & $\Lambda_{c}^{+} \rightarrow \Sigma^{+} \pi^{0} \pi^{0} \pi^{-} \pi^{+}$ & 0.01243 \\
\hline
\end{tabular}



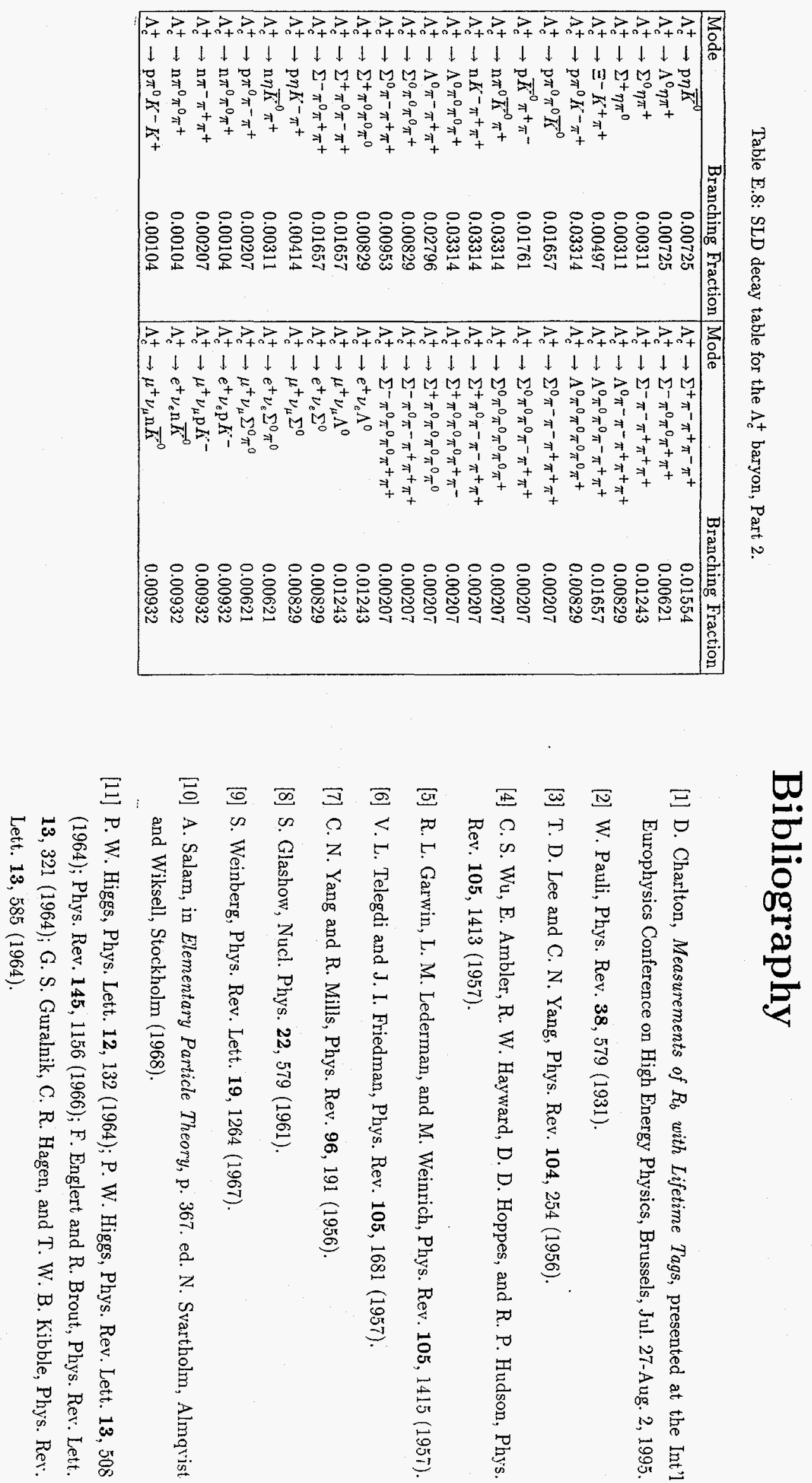
[12] G. t'Hooft, Nucl. Phys. B33, 173 (1971); G. t'Hooft, Nucl. Phys. B35, 167 (1971).

[13] F. J. Hasert et. al. (GARGAMELLE Collaboration), Phys. Lett. B46, 121 (1973); J. Blietschan et. al. (GARGAMELLE Collaboration), Nucl. Phys. B114, 189 (1976); J. Blietschan et. al. (GARGAMELLE Collaboration), Phys. Lett. B73, 232 (1978).

[14] For a review of accelerator-based neutrino scattering experiments, see D. H Perkins, Proceedings of the SLAC Summer Institute, SLAC-REPORT 215, p. 1 (1978)

[15] S. L. Glashow, J. Iliopoulos, and L. Maiani, Phys. Rev. D2, 1285 (1970).

[16] M. Perl et. al., Phys. Rev. Lett 35, 1489 (1975).

[17] W. R. Innes et. al., Phys. Rev. Lett 391240 (1977). Erratum: Phys. Rev. Lett 39, 1640 (1977).

[18] F. Abe et. al. (CDF Collaboration), FERMILAB-PUB-95-022-E (1995). Submitted to Phys. Rev. Lett.

[19] S. Abachi et. al. (D0 Collaboration), FERMILAB-PUB-95-028-E (1995). Submitted to Phys. Rev. Lett.

[20] G. Arnison et. al. (UA1 Collaboration), Phys. Lett. B134, 469 (1984).

[21] G. Arnison et. al. (UA1 Collaboration), Phys. Lett. B126, 398 (1983).

[22] F. Halzen and A. Martin, Quarks and Leptons: an Introductory Course in Modern Particle Physics, John Wiley and Sons, New York (1984).

[23] D. H. Perkins, Introduction to High Energy Physics, Third Edition, AddisonWesley, Menlo Park, California (1987).

[24] M. E. Peskin and D. V. Schroeder, An Introduction to Quantum Field Theory, Addison-Wesley, Menlo Park, California, (1995).
[25] This limit is the one cited in the Review of Particle Properties, Full Listings, Phys. Rev. D50, p. 1351 (1994). This limit was obtained using the galactic magnetic field. The original reference is Chibisov et. al., Soviet Physics - Uspekhi 19624 (1976).

E. Fischbach, H. Kloor, R. A. Langel, A. T. Y. Liu, and M. Peredo, Phys. Rev. Lett. 73, 514 (1994).

[26] Y. Nambu, Phys. Rev. Lett 4, 380 (1960); J. Goldstone, A. Salam, and S. Weinberg, Phys. Rev 127, 965 (1962).

[27] T.-P. Cheng and L.-F. Li, Guage Theory of Elementary Particle Physics, Clarendon Press, Oxford (1984).

[28] D. J. Gross and F. Wilczek, Phys. Rev. Lett. 30, 1343 (1973); D. J. Gross and F. Wilczek, Phys. Rev. D8, 3633 (1973).

[29] R. D. Field and R. P. Feymman, Nucl. Phys. B136, 1 (1978).

[30] T. Sjöstrand, Computer Phys. Commun. 39347 (1986); T. Sjöstrand and M. Bengtsson, Comput. Phys. Commun. 43367 (1987).

[31] T. Sjöstrand, CERN-TH.6488 (1992) (Version 7.3); T. Sjöstrand, CERN-TH7112-93, Feb, 1994 (Version 7.4)

[32] A. Kronfeld, FERMILAB-CONF-95/067-T (1995).

[33] J. Ellis, M. K. Gaillard, and G. G. Ross, Nucl. Phys. B111, 253 (1976).

[34] K. Abe et. al. (SLD Collaboration), Phys. Rev. Lett 71, 2528 (1993); K. Abe et. al. (SLD Collaboration), Phys. Rev. D50, 5580 (1994);

[35] S. J. Brodsky and G. P. Lepage, Phys. Rev. D28, 228 (1983) is a reference which proposes a procedure to elmiminate scale ambiguities in QCD. Also see H. J. Lu, Stanford Ph. D. Thesis, SLAC-REPORT-406 (1992).

[36] P. N. Burrows and H. Masuda, SLD Physics Note \# 36 (1995). 
[37] J. D. Bjorken, Phys. Rev. D17, 171, (1978).

[38] P. Clarke, RAL-90-055 (1990).

[39] A. Blondel, B. W. Lynn, F. M. Renard, and C. Verzegnassi, Nucl. Phys. B304, 438 (1988).

[40] F. M. Renard, A. Blondel, and C. Verzegnassi, CERN-88-06, Vol. 1, Polarization at LEP, 197 (1988).

[41] K. Abe et. al., Phys. Rev. Lett. 73, 25 (1994)

[42] E. Torrence, SLAC Experimental Seminar, June 8, 1995.

[43] P. Gambino and A. Sirlin, NYU-TH-93/09/07, (1993).

[44] D. Buskulic et. al. (ALEPH Collaboration), CERN-PPE-95-023 (1995), submitted to Z. Phys; M. Acciarrie et. al. (L3 Collaboration), Phys. Lett. B341, 245 (1994); R. Akers et. al. (OPAL Collaboration), Z. Phys. C65, 1 (199.5).

[45] D. Williams, Ph. D. Thesis, MIT (1994). Published as SLAC-REPORT-445.

[46] K. Abe et. al. (SLD Collaboration) Phys. Rev. Lett. 74, 2895 (1995).

[47] J. Huber, talk given at the Int'l Europhysics Conference on High Energy: Physics, Brussels, Jul. 27-Aug. 2, 1995. The SLD proceedings contribution is in preparation.

[48] K. Abe et. al. (SLD Collaboration) Phys. Rev. Lett. 74, 2890 (1995).

[49] K. Abe et. al. (SLD Collaboration) SLAC-PUB-6979, submitted to the Int"1 -. Europhysics Conference on High Energy Physics, Brussels, Jul. 27-Aug. 2, 1995.

[50] D. Su, Production of Charm and Beauty in $e^{+} e^{-}$with Polarized Electron Beam. Invited talk at the $6^{\text {th }}$ Int'l Symposium on Heavy Flavor Physics, Pisa, June 6 , 1995.

[51] D. Bardin et. al., CERN-TH-6443 (1992).
[52] M. Peskin, Proceedings of the $17^{\text {th }}$ SLAC Summer Institute on Particle Physics, SLAC-REPORT-361, p. 71 (1989).

[53] D. C. Kennedy, J. M. Im, B. W. Lynn, and R. G. Stuart, SLAC-PUB-4128 (1988).

[54] G. Altarelli and B. Lampe, Nucl. Phys. B391, 3 (1993).

[55] A. Djouadi, J. H. Kühn, and P. M. Zerwas, Z. Phys. C46, 411 (1990)

[56] B. A. Schumm, SLD Physics Note \# 23 (1993).

[57] A. Djouadi, B. Lampe, and P. M. Zerwas, MPI-PH-94-81 (1994).

[58] J. B. Stav and H. A. Olsen, Trondheim U. Preprint 1994-17, (1994). This work has been summarized by P. N. Burrows in SLD Physics Note \# 29 (1994).

[59] M. L. Swartz, SLAC-PUB-6710 (1994). Submitted to Phys. Rev. D.

[60] M. Peskin and T. Takeuchi, Phys. Rev. Lett 65, 964 (1990).

[61] M. Peskin and T. Takeuchi, Phys. Rev. D46, 381 (1992).

[62] M. Boulware and D. Finnell, Phys. Rev. D44, 2054 (1991).

[63] T. G. Rizzo, SLAC-PUB-6672 (1994). Submitted to Phys. Rev. D.

[64] A. Olchevski, Precision Tests of the Standard Model, presented at the Int.1 Europhysics Conference on High Energy Physics, Brussels, Jul. 27-Aug. 2, 1995.

[65] A. Passeri, Forward-Backward Asymmetris for Heavy Flavours using Lifetime - Tags, presented at the Int'l Europhysics Conference on High Energy Physics. Brussels, Jul. 27-Aug. 2, 1995.

[66] R. Akers et. al. (OPAL Collaboration), CERN-PPE/95/50 (1995).

[67] D. Buskulic et. al. (ALEPH Collaboration), Phys. Lett. B335 99 (1994).

[68] P. Abreu et. al. (DELPHI Collaboration), Z. Phys. C65, 569 (1995) 
[69] F. Liu et. al. (AMY Collaboration), Phys. Rev. D49, 4339-4347 (1994).

[70] M. Shirakata et. al. (VENUS Collaboration), Phys. Lett. B278, 499-505 (1992).

[71] K. Nagai et. al. (TOPAZ Collaboration), Phys. Lett. B278, 506-510 (1992).

[72] SLAC Linear Collider Conceptual Design Report, SLAC-REPORT-229 (1980). This report contains much that is out-dated, but provides an overview of the design considerations of the major SLC components.

[73] R. Prepost, Proceedings of the $21^{\text {st }}$ SLAC Summer Institute on Particle Physics, SLAC-REPORT-444, p. 57 (1994).

[74] Neal, Richard B., The Stanford Two Mile Accelerator, Benjamin, N.Y. (1968).

[75] Z. D. Farkas, SLAC SLED Program, SLAC-TN-77-001, April, 1977; Z. D. Farkas, H. A. Hogg, G. A. Loew and A. R. Wilmunder, IEEE Trans. Nucl. Sci. NS-24, No. 3, 1827; G. A. Loew, Proc $\mathrm{X}^{t} h$ Int. conf. on High Energy Accel., Serpukhov, USSR (1977), Vol 1, p. 58. See also Reference [72], p. 13-16.

[76] H. Wiedemann, SLAC-CN-58 (1981).

[77] C. Simopoulos and R. L. Holtzapple, SLAC-PUB-6813 (1995).

[78] C. Adolphsen /eal/ Proceedings of the IEEE Particle Accelerator Conference. p. 2019 (1993).

[79] K. Bane et. al., SLAC-PUB-6894 (1995). Presented at the IEEE Particle Accelerator Conference (PAC 95).

[80† F. J. Decker, R. Holtzapple, T. Raubenheimer, SLAC-PUB-6604 (1994). Presented at the $17^{\text {th }}$ International Linear Accelerator Conference (LINAC 94), Tsukuba, Japan, 1994.

[81] L. Hendrickson et. al., SLAC-PUB-6621 (1994). Presented at the $17^{\text {th }}$ International Linear Accelerator Conference (LINAC 94), Tsukuba, Japan, 1994.
[82] R. Erickson, T. H. Feiguth, J. J. Murray, SLAC-PUB-4199 (1987). Published in Washington PAC, p. 142 (1987).

[83] F. Zimmermann et. al., SLAC-PUB-6790 (1995). Presented at the IEEE Particle Accelerator Conference (PAC 95).

[84] G. Bonvicini, R. Frey, E. Gero, W. A. Koska, R. C. Field, and A. Minten, Nucl. Instr. Meth. A277, 297 (1989).

[85] Y.-C. Chao et. al, SLAC-PUB-5868 (1992). Presented at the $15^{\text {th }}$ Int'l conf. on High-Energy Accelerators, Hamburg, Germany, Jul 20-24, 1992.

[86] S. Wagner, Proceedings of the May 12-14, 1993 SLD Collaboration Meeting, Volume 2, Chateau La Cresta, Saratoga, CA (1993); S. Wagner, Proceedings of the February 7-9, 1994 SLD Collaboration Meeting, Volume 2, Chateau La Cresta, Saratoga, CA (1994); and S. Wagner, Proceedings of the February 1517, 1995 SLD Collaboration Meeting, Volume 2, p. 705, Chateau La Cresta, Saratoga, CA (1993).

[87] A. A. Sokolov and I. M. Ternov, Sov. Phys. Doklady 8, 1203 (1964).

[88] W. Heitler, The Quantum Theory of Radiation, $3^{\text {Td }}$ ed., p. 264-267. Clarendon Press, Oxford. Reprinted by Dover, New York.

[89] P. Horowitz and W. Hill, The Art of Electronics, p. 437-442, Cambridge University Press, Cambridge (1980).

[90] T. Junk, SLD Note \# 227 (1992).

[91] T. Lindberg, P. Emma, and R. Rossmanith, Proceedings of the IEEE Particle Accelerator Conference, p. 429 (1993).

[92] J. Irwin, Proceedings of the $21^{\text {st }}$ SLAC Summer Institute on Particle Physics. SLAC-REPORT-444, p. 209 (1994).

[93] D. Calloway et. al., SLAC-PUB-6423 (1994). 
[94] T. Junk, SLD Note \# 245 (1995).

[95] A. Lath and M. Woods, SLD Note \# 236 (1994).

[96] O. Klein and Y. Nishima, Z. Phys. 52, 823 (1929); Y. Nishina, Z. Phys. 52, 869 (1929).

[97] H. Veltman, Phys. Rev. D40, 2810 (1989).

[98] W. Nelson, H. Hirayama, D. W. O. Rogers, SLAC-REPORT-265 (1985)

[99] B. A. Schumm (private communication).

[100] A. Lath, D. Freytag, Compton Analysis: LeCroy Model 2249W ADC Linearity Study, SLD Note 237, April, 1994.

[101] M. Swartz et. al., SLAC-PUB-6467 (1994), submitted to Nucl. Instrum. Meth.

[102] L. G. Levchuk, Kharkov Preprint KFTI 92-32 (1992); L. G. Levchuk, Nucl. Instr. Meth. A345, 496 (1994).

[103] M. S. Lubell, Proceedings of the High Energy Physics with Polarized Beams and Targets, Argonne, IL, 1976 p. 418-423.

[104] G. Blaylock, SLD Physics Note \# 22 (1993). Also, G. Blaylock, D. Briggs, B. Collins, and M. Petree, SLAC-PUB-5649 (1992). Presented at the Technical Conf. and Expo, San Francisco, CA, Jan 15-17, 1992.

[105] SLD Design Report, SLAC-REPORT-273 (1984).

[106] S. C. Berridge et. al., IEEE Trans. Nucl. Sci. 37, p. 1191 (1990).

[107] K. T. Pitts, SLAC-REPORT-446, Electroweak Coupling Measurements from Polarized Bhabha Scattering at the $Z^{0}$ Resonance, $\mathrm{Ph}$. D. Thesis, University of Oregon (1994).

[108] M. Hildreth et. al., SLAC-PUB-6656, Sept, 1994 (submitted to IEEE Trans. Nucl. Sci.).
[109] T. Usher, Proceedings of the SLD Collaboration Meeting, April 9-11, 1992, Vol 2 (1992)

[110] D. Williams, Ph. D. Thesis, MIT (1994), Appendix B. Published as SLAC REPORT-445.

[111] G. Agnew et. al., SLAC-PUB-5906 (1992).

[112] M. Strauss et. al., Proceedings of the $7^{\text {th }}$ Meeting of the APS Division of Particles and Fields, World Scientific, Singapore, p. 1758 (1992). Also printed as SLAC-PUB- 5970.

[113] P. Billoir, Nucl. Instr. Meth. 225352 (1984).

[114] M. Cavalli-Sforza et. al., IEEE Trans. Nucl. Sci. 37, 1132 (1990).

[115] D. Axen et. al., Nucl. Inst. and Meth. A238, 472 (1993).

[116] A. C. Benvenuti et. al., Nucl. Instrum. Meth. A290, 353 (1990).

[117] E. Iarocci, Nucl. Instrum. Meth. 217, 30 (1983). G. Battistoni et. al., Nucl. Instrum. Meth. 217, 429 (1983).

[118] C. Peterson, D. Schlatter, I. Schmitt, and P. M. Zerwas, Phys. Rev. D27, 105 (1983).

[119] B. A. Schumm, Proceedings of the May 12-14, 1993 SLD Collaboration Meeting Chateau La Cresta (1993).

[120] R. Wang, Ph. D. Thesis, University of Minnesota (1994).

[121] N. Isgur, D. Scora, B. Grinstein, and M. B. Wise, Phys. Rev. D39, 799 (1989)

[122] H. Albrecht et. al. (ARGUS Collaboration), Phys. Lett. B221, 422 (1989); H Albrecht et. al. (ARGUS Collaboration), Phys. Lett. B232, 398 (1989).

[123] P. Kim (private communication, 1994)

[124] H. Albrecht et. al. (ARGUS Collaboration), Z. Phys. C58, 191 (1993). 
[125] H. Albrecht et. al. (ARGUS Collaboration), Z. Phys. C54, 13 (1992).

[126] D. Coffman et. al. (MARK III Collaboration), Phys. Lett. B263, 135.

[127] F. Muheim, Presented at the 1994 APS DPF Meeting, Santa Fe, NM (1994).

[128] M. Thulasidas, Ph. D. Thesis, Syracuse University (1993).

[129] D. Su (private communication).

[130] GEANT program, version 3.21, CERN Application Software Group, CERN Program Library (1993). See R. Brun et. al., GEANT3 User's Guide, CERNDD//EE/84-1, CERN (1989).

[131] H. Fesefeldt, GHEISHA, PITHA 85/02 (1985).

[132] G. Grindhammer et. al., Nucl. Instr. Meth. A290, 469 (1990).

[133] See, for example, the PDG's mini-review of electromagnetic shower parameterization in Physical Review D50, 1255-1256, and references therein.

[134] K. Abe et. al, SLAC-PUB-6569, July, 1994 (submitted to Phys. Rev. D).

[135] R. G. Jacobsen, Ph.D. Thesis, Stanford University, 1991. Published as SLACREPORT-381.

[136] E. Farhi, Phys. Rev. Lett 39, 1587 (1977).

[137] R. Brandelik et. al. (TASSO Collaboration), Phys. Lett. B100 357 (1981); C. Berger et. al. (PLUTO Collaboration), Nucl. Phys. B214 189 (1983); W: W. Ash et. al. (MAC Collaboration), Phys. Rev. Lett. 581080 (1987); T. Greenshaw et. al. (JADE Collaboration), Z. Phys. C42 1 (1989).

[138] D. Stuart et. al. (AMY Collaboration), Phys. Rev. Lett. 64983 (1990); D. Stuart et. al. (AMY Collaboration); M. Shirakata et. al. (VENUS Collaboration), Phys. Lett. B278, 499, (1992).

[139] D. Buskulic et. al. (ALEPH Collaboration), Phys. Lett. B284, 177 (1992); P. D. Acton et. al. (OPAL Collaboration) Phys. Lett. B294, 436 (1992).

[140] M. Fendt et. al. (DELPHI Collaboration), CERN-PPE-95-076 (1995). Contributed to the 1995 Rencontres de Moriond Electroweak Session.

[141] P. D. Acton et. al. (OPAL Collaboration) Phys. Lett. B302, 523 (1993)

[142] M. V. Purohit et. al. (E791 Collaboration), FERMILAB-CONF-94-186-E (1994). Contributed to 27th International Conference on High Energy Physics (ICHEP), Glasgow, Scotland, 20-27 Jul 1994.

[143] P. N. Burrows, H. Park, K. t. Pitts, and J. M. Yamartino, SLD Note 229 (199:3).

[144] J. M. Yamartino, SLD Physics Note 14 (1992).

[145] A review of SLC backgrounds may be found in M. D. Hildreth, Ph. D. Thesis, SLAC-REPORT-95-458, Appendix D, p. 300-324 (1995).

[146] M. Aguilar-Benitez et. al., Physical Review D50, 1173 (1994).

[147] H. Neal, Stanford University Ph. D. Thesis (1995). Published as SLACREPORT-473

[148] Jets are defined with the JADE algorithm, W. Bartel et. al., Z. Phys. C33. 23 (1986), using a value of $y_{\min }=0.02$.

[149] D. Su, Proceedings of the February 7-9, 1994 SLD Collaboration Meeting, Volume 2, Chateau La Cresta, Saratoga, CA (1994).

[150] D. Decamp et. al. (ALEPH Collaboration), Phys. Lett. B273, 181 (1991).

[151] M. Hildreth, Stanford Ph.D. Thesis, p. 123 (1995). Published as SLACREPORT-458.

[152] B. Lampe, MPI-PH-93-74 (1993). 
[153] M. Fero, SLAC-PUB-6678 (1994), presented at the XIV International Conference on Physics in Collision, Tallahassee, Florida, June 15-17, 1994.

[154] M. G. Bowler, Z. Phys. C11 161 (1981).

[155] D. Abbaneo, presented at the 1994 Rencontres de Moriond, Electroweak Session, March 12-19, 1994.

[156] D. Decamp, et. al. (ALEPH Collaboration), Phys. Lett. B278, 209 (1992).

[157] P. D. Acton, et. al. (OPAL Collaboration), Phys. Lett. B281 394 (1992).

[158] K. Abe et. al. (SLD Collaboration), Phys. Rev. Lett. 72, 3145 (1994).

[159] S. Komahiya, $b$ and $c$ Physics, presented at the Int'l Europhysics Conference on High Energy Physics, Brussels, Jul. 27-Aug. 2, 1995.

[160] A. F. Falk and M. E. Peskin, Phys. Rev. D49 3320 (1994).

[161] T. Mannel and G. A. Schuler, Phys. Lett. B279, 194 (1992).

[162] F. E. Close, J. Körner, R. J. N. Phillips, and D. J. Summers, J. Phys. G18, $1716(1992)$

[163] J. G. Körner and M. Krämer, Phys. Lett. B275, 495 (1992)

[164] ALEPH Collaboration, EPS-0400, internal note contributed to the Int'l Europhysics Conference on High Energy Physics, Brussels, Jul. 27-Aug. 2, 1995.

[165] W. T. Eadie, D. Drijard, F. E. James, M. Roos, and B. Sadoulet, Statistical Methods in Experimental Physics, American Elsevier, 1971.

[166] See, for example, the PDG's mini-review of maximum-likelihood techniques in Physical Review D50, 1275-1282, and references therein.

[167] P. Billingsley, Probability and Measure, $2^{\text {nd }}$ ed., John Wiley \& Sons, New York (1986), or any advanced text on statistics.
[168] H. Quinn, SLAC-PUB-6438 (1994); Decays into non-CP eigenmodes may have some asymmetry by projection onto $\mathrm{CP}$ eigenmodes with a helicity analysis see I. Dunietz, H. Quinn, A. Snyder, and W. Toki

[169] G. Marchenisi, B. R. Webber, G. Abbiendi, I. G. Knowles, M. H. Seymour, and L. Stanco, Comput. Phys. Commun. 67, 465-508 (1992).

[170] T. Takeuchi, A. K. Grant, J. L. Rosner, FERMILAB-CONF-94/279-T, presented at the DPF'94 Meeting, Albuquerque, NM. Aug 2-6, 1994.

[171] M. Swartz, SLAC-PUB-7001, Sep, 1995 (Submitted to Phys. Rev. D).

[172] B. Schumm, talk presented at the SLD Collaboration Meeting, August, 1995, Kirkwood, CA.

[173] D. Comelli, C. Verzegnassi, and F. M. Renard, UTS-DFT-94-02 (1994).

[174] D. Strom, Precision Electroweak Experiments at LEP, Invited talk at the SLAC Summer Institute, July 10-21, 1995. (get a better ref from EPS).

[175] M. Hildreth, Stanford Ph.D. Thesis (1995). Published as SLAC-REPORT-458.

[176] K. Abe et. al. (SLD Collaboration), SLAC-PUB-6681 (1995).

[177] D. Buskulic et. al. (ALEPH Collaboration), CERN-PPE-95-44 (1995); P. Abreu et. al. (DELPHI Collaboration), Z. Phys. C66, 341 (1994); R. Akers et. al. (OPAL Collaboration), CERN-PPE-94-217 (1994).

[178] P. Baringer et. al. (HRS Collaboration), Phys. Lett. B206, 551 (1988).

[179] A. Okamoto et. al. (VENUS Collaboration), Phys. Lett. B278 393 (1992).

[180] D. Jackson, SLD Physics Note \# 27 (1995).

[181] SLD Collaboration, VXD3 Note \# 1 (1994). 